\author{
Universidade de São Paulo \\ Instituto de Física
}

\title{
Aspectos físico-matemáticos no tratamento de lentes gravitacionais sobre a radiação cósmica de fundo
}

\author{
Paulo Henrique Flose Reimberg
}

Orientador: Prof. Dr. Luis Raul Weber Abramo

Tese de doutorado apresentada ao Instituto de Física para a obtenção do título de Doutor em Ciências

Prof. Dr. Luis Raul Weber Abramo (IFUSP)

Prof. Dr. Alberto Vazquez Saa (IFUSP)

Prof. Dr. João Carlos Alves Barata (IFUSP)

Prof. Dr. George Emanuel Avraam Matsas (IFT-UNESP)

Prof. Dr. Thiago dos Santos Pereira (UEL) 


\section{FICHA CATALOGRÁFICA}

\section{Preparada pelo Serviço de Biblioteca e Informação do Instituto de Física da Universidade de São Paulo}

Reimberg, Paulo Henrique Flose

Aspectos físicos-matemáticos no tratamento de lentes gravitacionais sobre a radiação cósmica de fundo - São Paulo, 2013.

Tese (Doutorado) - Universidade de São Paulo. Instituto de Física. Depto. de Física Matemática

Orientador: Prof. Dr. Luis Raul Weber Abramo

Área de Concentração: Física-matemática

Unitermos: 1. Radiação de fundo; 2. Lentes gravitacionais;

3. Relatividade (Geometria diferencial); 4. Funções de Bessel.

USP/IF/SBI-111/2013 


\section{Agradecimentos}

Agradeço à FAPESP pelo apoio financeiro, ao Departamento de Física Matemática do IFUSP e Institut de Physique Théorique do CEA-Saclay pela hospitalidade e a João Barata e Walter Wreszinski pelas discussões. 


\section{Resumo}

A hierarquia de equações de Boltzmann que descreve a temperatura e polarização da radiação cósmica de fundo é tratada no espaço das posições. Mostramos neste formalismo que temperatura e polarização podem ser descritas como médias dos termos de fonte ponderados por probabilidades associadas a um problema de vôos aleatórios. Decorre da estrutura geral da hierarquia que se pode fazer uma expansão da temperatura e polarização em termos do número de espalhamentos ocorridos durante a recombinação. Incorporamos o efeito de lentes gravitacionais sobre a radiação cósmica de fundo tirando proveito da estrutura das equações no espaço das posições. Mostraremos que o efeito é incorporado através de correções aos coeficientes da decomposição angular dos campos de temperatura e polarização. Para descrever o efeito de lentes gravitacionais fazemos uma revisão sobre resultados formais da teoria e apresentamos uma rederivação de equações centrais em espaços-tempo arbitrários. 


\begin{abstract}
The Boltzmann hierarchy describing the temperature and polarization of the cosmic microwave background is presented in a position space formalism. We show that temperature and polarization can be described in terms of averages over source terms weighted by probabilities densities that appear in problems of random flights. The temperature and polarization signals can be expanded in terms of the number of scatterings photons suffered during the recombination. The gravitational lensing effects are incorporated over the free-propagation phase of the CMB photons. This effect can be included in the position space formalism as a correction to the expansion coefficients of the temperature and polarization fields. The bases of the theory of gravitational lensing are also presented and a rederivation of the central equations of the theory in arbitrary spacetimes is here developed.
\end{abstract}





\section{Conteúdo}

1 Lentes Gravitacionais: maquinaria básica 5

1.1 Princípio de Fermat: Bases 8

1.2 Caráter dos pontos estacionários 16

1.3 Pontos conjugados e imagens múltiplas 22

1.4 Lentes gravitacionais, pontos críticos e topologia 27

2 Evolução de feixes de geodésicas nulas e lentes gravitacionais

2.1 O problema da derivação de [1]: interpretações da equação de Jacobi

2.2 O espaço de vetores adequado para o estudo de nosso problema

2.3 Restrição da métrica, derivada e curvatura

2.4 Classes de Jacobi

2.5 Tensores de Jacobi

2.6 Equação de Raychaudhuri para vetores nulos, condições de energia e pontos conjugados

2.7 Equações centrais da teoria de Lentes Gravitacionais 48

2.8 Aplicação: O mapa de Jacobi no caso cosmológico 52

3 Equações de Boltzmann para temperatura e polarização da CMB no espaço das posições

3.1 As equações de Boltzmann

3.2 Estudo do sistema quando se toma $P=0$ na temperatura

3.3 Conexão entre a expressão da polarização e processos de vôos aleatórios

3.4 Retorno à descrição da polarização quando $P=0 \quad 74$

3.5 Diagramas 76

3.6 Reintrodução de fontes de polarização na temperatura $\quad 77$

3.7 Domínios de dependência 86

3.8 Possível forma de cálculo das densidades de probabilidade $\quad 88$ 
6 Conteúdo

3.9 Considerações sobre convergência

4 Efeito de lentes fracas sobre a temperatura e polarização da CMB: estudo no espaço das posições 93

4.1 Tratamento da temperatura 93

$\begin{array}{lll}4.2 & \text { Tratamento da polarização } & 97\end{array}$

$\begin{array}{lll}4.3 & \text { O efeito do potencial de lente } & 101\end{array}$

$\begin{array}{lll}4.4 \text { Resumo } & 104\end{array}$

Apêndice A Teoremário $\quad 111$

A.1 Geometria de vetores nulos 111

$\begin{array}{lll}\text { A.2 Estrutura causal } & 113\end{array}$

$\begin{array}{ll}\text { A.3 Conexões } & 116\end{array}$

$\begin{array}{ll}\text { A.4 Curvaturas } & 117\end{array}$

A.5 Bases não-coordenadas e equações de estrutura de Cartan 121

A.6 Geodésicas 123

A.7 Campos de Jacobi 124

Apêndice B Trajetórias em espaços-tempo perturbados 127

Apêndice C Alguns resultados sobre funções de Bessel 131

C.1 Primeiro grupo de resultados: Relação entre integrais de produtos de funções de Bessel e vôos aleatórios

C.2 Segundo grupo de resultados: Ampliação da família de integrais que podem ser relacionadas a vôos aleatórios 143

C.3 Terceiro grupo de resultados: séries de Fourier-Bessel 146

C.4 Quarto grupo de resultados: uma relação tipo-completeza para funções de Bessel esféricas 148

Apêndice D Funções de spin e geometria na esfera

D.1 Representações irredutíveis unitárias de $\mathrm{SO}(3)$ e funções de spin

D.2 Símbolos de Christoffel na esfera 160

D.3 Maneira de reescrever a derivada covariante 161

D.4 Relações envolvendo $D_{m m^{\prime}}^{j}$ s e coeficientes de Clebsch-Gordan

163

Referências Bibliográficas 


\section{Introdução}

Consideremos um fliperama: uma partícula com posição e momento iniciais definidos interage através das leis de Newton com um conjunto de centros espalhadores esfericamente simétricos contidos em uma região limitada do espaço. Ao fazer o raio dos centros espalhadores progressivamente menor e aumentar ao mesmo tempo a sua densidade, pode-se $\operatorname{mostrar}^{1}$ que o processo estocástico converge a um vôo aleatório, ou seja, o movimento realizado por um caminhante com velocidade constante, que muda sua direção de movimento em instantes aleatoriamente sorteados.

Parte da dinâmica da radiação cósmica de fundo $\left(\mathrm{CMB}^{2}\right)$ pode ser traduzida nas características deste fliperama: antes de afastarem-se uma certa distância dada da origem do movimento, os caminhantes (supondo que se jogue nesse fliperama um número grande de vezes) podem ter sofrido nenhum, um, dois, alguns poucos, ou um número indefinidamente grande de colisões com os obstáculos e há uma probabilidade associada a este afastamento da origem para cada número de colisões. No caso da radiação cósmica de fundo, os mesmos pesos probabilísticos são associados à história de fótons que se espalharam um dado número de vezes com elétrons durante a recombinação.

Em cada um dos espalhamentos sofridos, os fótons têm sua energia e polarização modificadas e os observáveis finais carregam as informações dos meios onde as interações deram-se. Quando se aumenta o número de espalhamentos sofridos, no entanto, o teorema $\mathrm{H}$ de Boltzmann afirma que ficamos mais próximos do equilíbrio entre radiação e matéria e consequentemente o peso da história desses fótons deve ser reduzido na contribuição final para as flutuações de temperatura e polarização líquida da CMB. Da mesma maneira, se nenhum espalhamento ocorresse, teríamos o limite de recombinação instantânea, em que os fótons carregam apenas as características da superfície de último espalhamento e nenhuma polarização é gerada. Mostraremos ser possível estabelecer uma expansão da temperatura e polarização da CMB em termos do número de

\footnotetext{
${ }^{1}[2]$ mostra que no limite de Boltzmann-Grad um processo de Lorentz converge a um vôo aleatório.

${ }^{2}$ Sigla em inglês para Cosmic Microwave Background.
} 


\section{Introdução}

espalhamentos sofridos durante a recombinação. A descrição das flutuações de temperatura e polarização da radiação será dada através de uma média sobre termos de fonte com pesos probabilísticos relacionados a vôos aleatórios para cada possível número de espalhamentos. A maneira de obter-se este conjunto de médias a partir das equações de Boltzmann que descrevem o sistema colisional de fótons e elétrons durante a recombinação é apresentada no Capítulo 3 deste texto e deu origem à publicação [3]. As conclusões apresentadas neste Capítulo estão também parcialmente contidas em [4], que inicia o estudo de um formalismo causal para a CMB.

Após os espalhamentos sofridos durante a recombinação, através dos quais são impressas flutuações na distribuição de equilíbrio dos fótons, há propagação livre. Durante esta fase, no entanto, há ainda efeitos gravitacionais sobre as flutuações de temperatura e polarização: a temperatura dos fótons é afetada pela evolução temporal dos potenciais gravitacionais cosmológicos, temperatura e polarização são afetadas pelo efeito de lente gravitacional exercido por esses potenciais.

Os exemplos que vêm mais imediatamente à mente quando pensamos em lentes gravitacionais são os cenários clássicos em que a trajetória dos raios de luz são curvados em um espaço-tempo de Schwarzschild. Nesses casos conhecemos a concentração de massa, as condições assintóticas no espaço-tempo e as geodésicas nulas, podendo calcular completamente o efeito. Assumindo que as dimensões da concentração de massa sejam muito inferiores às outras distâncias envolvidas no problema, podemos parametrizar o efeito de distorção pelas distâncias típicas, massa do defletor e parâmetro de impacto. No caso das lentes causadas pelos potenciais cosmológicos, conhecer seu efeito passa também por determinar quais são as geodésicas nulas no espaço-tempo e saber como um feixe de tais geodésicas vizinhas deforma-se pelo efeito da gravitação. Não é evidente, entretanto, que uma descrição simplificada como a que se processa no caso de Schwarzschild seja possível. Decidimos então estudar o efeito a partir das equações gerais para lentes gravitacionais em espaços-tempo arbitrários e particularizá-lo para o problema específico do modelo cosmológico adotado. Notamos, entretanto, uma falha nas derivações dessas equações gerais apresentadas na referência clássica sobre o assunto. Tomando a rigor o que é apresentado em [1] concluiríamos que o efeito de lente gravitacional é sempre trivial. 
Com o intuito de rever o argumento de [1], que é assunto do Capítulo 2 do presente texto e deu origem à publicação [5], decidimos fazer um estudo mais detalhado do problema de lentes gravitacionais, o que é tema do Capítulo 1. Nosso ponto de partida é a formulação do Princípio de Fermat em Relatividade Geral. Caracterizaremos as geodésicas nulas como extremos de um funcional e mostraremos que esses extremos podem ser mínimos ou pontos de sela deste funcional, dependendo da existência de pontos em que geodésicas emergindo de um mesmo ponto voltem a cruzar-se ao longo da geodésica central do feixe. A existência de tais pontos, chamados de pontos conjugados, é condição suficiente para o surgimento de uma imagem múltipla da fonte. O número e características de pontos conjugados ao longo de uma geodésica são relacionados à natureza topológica do espaço de curvas ligando observador e fonte e uma série de resultados bastante gerais na teoria das lentes gravitacionais decorrem deste fato.

Após a revisão da literatura sobre resultados formais da teoria de lentes gravitacionais apresentada no Capítulo 1, apresentaremos a revisão do argumento de [1] no Capítulo 2. Aplicaremos as equações gerais derivadas para descrever lentes em espaços-tempos arbitrários para o caso específico do espaçotempo de Friedmann-Lemaître-Robertson-Walker sujeito a perturbações escalares. Graças às particularidades da solução obtida, escreveremos o mapa de lentes no caso cosmológico.

Em posse do mapa de lente e da descrição da hierarquia de Boltzmann no espaço das posições, podemos tratar o problema das lentes gravitacionais sobre a temperatura e polarização da CMB. Mostraremos no Capítulo 4 como incorporar o efeito dos potenciais gravitacionais entre as integrais que descrevem os espalhamentos sofridos pelos fótons durante a recombinação. Mostraremos também qual a ação das lentes gravitacionais sobre a parte angular das expansões dos campos de temperatura e polarização. Veremos que o efeito das lentes gravitacionais pode ser incorporado dentro da mesma estrutura de decomposição dos campos através de uma correção aos coeficientes das expansões.

Uma série de apêndices complementa o texto. Todos exceto um são meras revisões de resultados conhecidos mas que se julgou conveniente reunir para facilitar a leitura do texto principal. O Apêndice C, no entanto, é contribuição original deste trabalho e dedica-se a estudar uma classe de integrais de produtos de funções de Bessel esféricas que surge naturalmente no estudo das flutuações 


\section{Introdução}

de temperatura e polarização da CMB no espaço das posições. Mostraremos nesse apêndice a conexão das integrais citadas e o problema de vôos aleatórios. Demonstraremos também nesse apêndice uma relação satisfeita por funções de Bessel esféricas que fora conjecturada em [4] e que não era conhecida na literatura. A demonstração deu origem a [6]. 


\section{1}

\section{Lentes Gravitacionais: maquinaria básica}

Dado um espaço-tempo ${ }^{1}(M, g)$, tomemos um ponto $p \in M$ e uma curva $C^{\infty}$ tipo-tempo $\eta:[0, a] \rightarrow M$, que serão interpretados respectivamente como o ponto de observação (um telescópio aqui e agora apontado para o céu) e a linha de mundo de uma fonte luminosa pontual, como um quasar distante, por exemplo. Dados estes elementos pergunta-se como será vista a fonte luminosa pelo observador, ou seja, qual a natureza das geodésicas nulas dirigidas ao passado que conectam o ponto $p$ à curva $\eta$.

Em relatividade geral geodésicas nulas são interpretadas como raios de luz viajando sob a influência gravitacional de distribuições de matéria e/ou energia no espaço-tempo ${ }^{2}$. Assim, cada geodésica nula apontando para o passado de $p$ a $\eta$ corresponde a uma imagem da fonte luminosa vista na esfera celeste pelo observador $p$. Caracterizam-se as configurações de imagem de uma fonte para um observador catalogando-se as geodésicas nulas dirigidas ao passado ligandoos, dados o conteúdo material, as condições assintóticas impostas à métrica e assumindo-se a teoria da relatividade geral.

Antes de seguir, observamos que um conjunto de definições e resultados sobre geometria e estrutura causal estão reunidos no Apêndice A e sua consulta pode facilitar a leitura deste capítulo.

Pode-se classificar em quatro casos as configurações de imagens de uma fonte pontual por um observador [7]:

i) Não há geodésicas nulas apontando para o passado de $p$ a $\eta$ : o observador $p$ não vê imagem alguma da fonte. No Universo de Gödel, por exemplo,

\footnotetext{
${ }^{1}$ Entendemos por espaço-tempo uma variedade Lorentziana $(M, g)$ quadridimensional, conexa e dotada de orientação temporal.

${ }^{2}$ Distribuições de matéria e/ou energia que causem curvatura no espaço-tempo são chamados, no contexto de lentes gravitacionais de defletores ou lentes devido sua analogia com lentes da óptica
} clássica. 
6 Lentes Gravitacionais: maquinaria básica

dois pontos podem ser conectados por uma curva causal apontando para o passado, mas as geodésicas nulas partindo de um ponto são restritas a regiões cilíndricas ( ver [8]).

ii) Há exatamente uma geodésica nula apontando para o passado ligando p a $\eta$. Neste caso o observador vê apenas uma imagem da fonte $\eta$. Este caso corresponde ao caso em que lentes tratadas de maneira geral em relatividade geral conduzem apenas a uma imagem do objeto observado e que é conhecido como limite de lentes fracas.

iii) Existem pelo menos duas geodésicas nulas, mas não mais do que um número contável delas, apontando para o passado de $p$ a $\eta$. O observador $p$ verá portanto, um número finito ou infinito contável de imagens distintas de $\eta$ em sua esfera celeste. De maneira geral as diferentes geodésicas nulas de $p$ a $\eta$ intersectam a linha de mundo de $\eta$ em pontos diferentes e, com isso, as imagens vistas têm "diferentes idades".

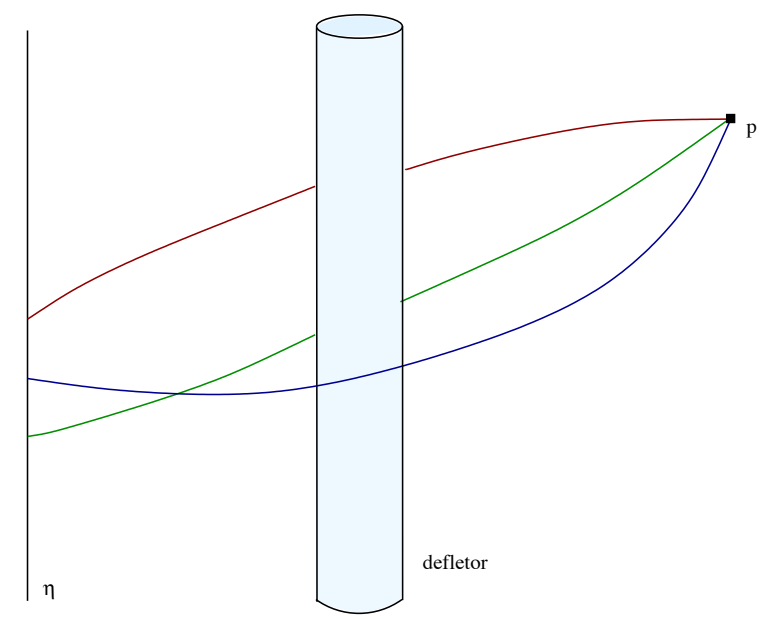

Fig. 1.1 Diagrama representando um caso de imagens múltiplas. Cada geodésica nula apontando para o passado deve corresponder a uma imagem vista pelo observador, a menos que alguma delas seja escondida pelo defletor.

iv) Existe um número incontável de geodésicas nulas apontando para o passado ligando $p$ a $\eta$. Neste caso pode existir, por exemplo, uma família uniparamétrica contínua de geodésicas nulas de $p$ a $\eta$ e a fonte $\eta$ aparecerá na esfera celeste de $p$ como um arco ou, em casos com simetria axial, 
como um anel (anel de Einstein). Pode-se mostrar que todos os membros de uma família uniparamétrica contínua de raios de luz conectando $p$ a $\eta$ necessariamente encontram $\eta$ no mesmo ponto, i. e., todas as partes de uma imagem extensa da fonte luminosa têm a mesma idade [7]. Vale notar que sob a hipótese de fonte e observador pontuais, a existência desse tipo de configuração de imagem é instável no sentido que desaparece mediante pequenas perturbações de $p$ mantendo $\eta$ fixa $^{3}$.

Decidir sobre o caso em que nos encontramos dado um cenário gravitacional requer método. Há naturalmente condições em que a solução das geodésicas nulas pode ser, dentro de limites de abrangência ou exatidão, completamente obtidas [10], [11]. Os casos em que soluções exatas são disponíveis, entretanto, são escassos e não necessariamente atrativos já que em geral sistemas físicos de interesse não são simples o suficiente para que a solução completa e explícita das geodésicas nulas seja possível. Evidencia-se então a necessidade de um método mais geral para o estudo do problema e por isso formularemos, seguindo [12], o princípio de Fermat para geodésicas nulas em variedades Lorentzianas. Estudaremos as características dos pontos críticos do funcional em que está fundamentado o princípio de Fermat e também as condições suficientes para que imagens múltiplas aconteçam. Embora muitos dos resultados formulados sejam bastante gerais, teremos nossa atenção restrita, neste momento, a espaços globalmente hiperbólicos.

No que tange a lentes gravitacionais, a restrição a espaços globalmente hiperbólicos significa ater-se a casos em que o defletor é transparente, em um espaço-tempo sem violações de causalidade e cuja topologia é o produto de subvariedades tipo-espaço (superfícies de Cauchy) e uma função tempo. Notamos ainda que estamos concentrados em espaço-tempos de dimensão igual a 4, ainda que muitos resultados sejam válidos para dimensão maior ou igual a três ${ }^{4}$.

\footnotetext{
${ }^{3} \mathrm{O}$ desaparecimento destas configurações com contínuos de imagens sob perturbações está ligada à afirmação do teorema de Morse para geodésicas nulas em variedades Lorentzianas [9], que diz que para tais geodésicas (em um dado intervalo) há um número contável de pontos conjugados e, portanto, dada uma curva tipo-tempo $\eta$, o conjunto de pontos $p \in M$ tal que $\gamma$ não encontra pontos conjugados no cone de luz passado de $p$ é denso em $M$.

${ }^{4}$ Geodésicas nulas em variedades Lorentzianas bidimensionais não têm pontos conjugados [9]. A implicação deste fato à teoria de lentes gravitacionais será evidente cedo na leitura deste texto.
} 


\section{Lentes Gravitacionais: maquinaria básica}

Notamos, antes de iniciar de fato os estudos variacionais, que existe uma simetria na formulação geral dos problemas de lentes gravitacionais no que diz respeito ao direcionamento temporal das geodésicas nulas: considerar geodésicas nulas apontando para o futuro emergindo de $\eta$ e alcançando $p$ é inteiramente equivalente a considerar geodésicas nulas apontando para o passado partindo de $p$ e interceptando $\eta$.

\subsection{Princípio de Fermat: Bases}

O princípio de Fermat em óptica fornece uma maneira não apenas elegante de formular suas leis básicas, mas também versátil o suficiente para permitir o tratamento de problemas complicados como propagação de luz em meios inhomogêneos [13], [14]. A existência de geodésicas nulas conectando eventos em espaços-tempo pode também ser refraseada em termos do princípio de Fermat, sendo este o instrumento central no estudo de óptica em espaços-tempo e, em especial, determinação do número de imagens de uma determinada fonte vistas por um observador.

Os resultados nesta seção são baseados em [12], [15], [16] e [17]. Utilizaremos a todo momento nestas seções que seguem resultados de geometria e relatividade geral reunidos no Apêndice A.

\subsubsection{Definições básicas}

Seja $\gamma(\lambda)$ uma curva em $M$ ligando os pontos $\gamma(0)=p$ a $\gamma(a)=q$.

Definição 1.1 A variação $f$ de $\gamma(\lambda)$ é uma aplicação contínua

$$
f:(-\epsilon, \epsilon) \times[0, a] \rightarrow M
$$

tal que

(i) $f(0, \lambda)=\gamma(\lambda)$;

(ii) Existe uma subdivisão $0=\lambda_{0}<\lambda_{1}<\ldots<\lambda_{n}=a$ de $[0, a]$ tal que $f$ é $C^{\infty}$ em cada $(-\epsilon, \epsilon) \times\left[\lambda_{i}, \lambda_{i+1}\right]^{5}$.

A variação é dita própria se $f(s, 0)=\gamma(0)$ e $f(s, a)=\gamma(a), s \in(-\epsilon, \epsilon)$. Para cada $s \in(-\epsilon, \epsilon)$ a curva parametrizada $f_{s}:[0, a] \rightarrow M$ dada por $f_{s}(\lambda)=f(s, \lambda)$

${ }^{5}$ Requerimentos menores na diferenciabilidade são possíveis - [15] pedem curvas $C^{3}$ por partes, por exemplo - mas não faremos tais restrições aqui. 
é chamada curva da variação. Chamaremos de curva transversal da variação a curva parametrizada diferenciável $f_{\lambda}:(-\epsilon, \epsilon) \rightarrow M, \lambda$ fixado, dada por $f_{\lambda}(s)=f(s, \lambda)$. O vetor velocidade de uma curva transversal em $s=0$ será chamado de campo variacional e será denotado por $V(\lambda)=\frac{\partial f}{\partial s}(0, \lambda)$.

Introduziremos agora o funcional que nos permitirá estudar o efeito de variações sobre curvas dadas. Diferentemente do que se faria no caso Riemanniano, ou ainda no caso de variações de curvas tipo-tempo, o funcional comprimento de $\operatorname{arco}^{6}$ é inadequado ao nosso intento já que não tem derivada sobre curvas nulas ${ }^{7}$. O que fazemos, então, é estudar o funcional energia (também chamado de ação) que, apesar de não ter significado geométrico direto, é computacionalmente mais simples, diretamente associado à interpretação da Forma do Índice (que será ainda nosso assunto) e, principalmente, aplica-se ao estudo de variações de curvas nulas ${ }^{8}$.

Definição 1.2 Chamaremos de funcional energia a função $E:(-\epsilon, \epsilon) \rightarrow \mathbb{R}$ dada por:

$$
E(s)=\int_{0}^{a}\left\langle\frac{\partial f}{\partial \lambda}(s, \lambda), \frac{\partial f}{\partial \lambda}(s, \lambda)\right\rangle d \lambda
$$

$\langle.,$.$\rangle denota a métrica no espaço-tempo. Convém notar que há uma diver-$ sidade de convenções possíveis para esta definição no que tange a fatores de $1 / 2$ ou sinais de menos, ver por exemplo [16] e [9]. Vale também observar que as equações de Euler-Lagrange para (1.1) são as equações de geodésica no espaço-tempo.

Se as variações de uma geodésica nula $\gamma$ são geodésicas nulas, então todas as derivadas do funcional energia anulam-se para $s=0$ para todos os possíveis campos variacionais, pois a energia é nula para todos eles. Somos, então levados a restringir nossa atenção à seguinte classe de variações:

$$
{ }^{6} L(s)=\int_{0}^{a} \sqrt{\left\langle\frac{\partial f}{\partial t}, \frac{\partial f}{\partial t}\right\rangle(s, t)} d t \operatorname{com} s \in(-\epsilon, \epsilon), t \in[0, a] .
$$

${ }^{7}$ Devemos notar que o problema variacional para curvas tipo-tempo pode também ser formulado em termos do funcional energia pois, se uma curva $\eta$ maximiza o comprimento de arco então, na parametrização afim, também maximiza $-E$ dado em (1.1).

${ }^{8} \mathrm{Em}$ outros contextos, como por exemplo [18], escolhe-se espaços-tempo globalmente hiperbólicos e, beneficiando-se da separabilidade da métrica, escreve-se uma curva nula como $\gamma(\lambda)=\left(c_{1}(\lambda), c_{2}(\lambda)\right)$ e considera-se a projeção no segundo fator definido como $J(\gamma)=\int\left[c^{\prime}(\lambda)\right]^{2} d \lambda$ como o funcional adequado ao estudo da Teoria do Índice. [19] emprega definição semelhante para o estudo da Teoria do Índice em espaços estavelmente causais. 
10 Lentes Gravitacionais: maquinaria básica

Definição 1.3 Uma variação $f$ de uma curva causal $\gamma$ (como descrita na definição 1.1) é dita ser admissível se todas as curvas vizinhas $f(s, \lambda)$ são tipotempo para qualquer s em $(-\epsilon, \epsilon)$.

No que segue, entenderemos variações como variações admissíveis.

\subsubsection{A primeira variação da energia}

Definição 1.4 Uma curva $\gamma$ é dita ser estacionária se e somente se $E^{\prime}(0)=0$ para toda variação $f$ de $\gamma$.

Proposição 1.5 Sejam $\gamma:[0, a] \rightarrow M$ uma curva diferenciável por partes $e$ $f:(-\epsilon, \epsilon) \times[0, a] \rightarrow M$ uma variação de $\gamma, 0=\lambda_{0}<\lambda_{1}<\ldots<\lambda_{n+1}=a$. Se $E:(-\epsilon, \epsilon) \rightarrow \mathbb{R}$ é a energia de $f$, então

$$
\begin{aligned}
\frac{1}{2} E^{\prime}(0)= & -\int_{0}^{a}\left\langle V(\lambda), \frac{D}{d \lambda} \frac{d \gamma}{d \lambda}\right\rangle d \lambda-\sum_{i=1}^{n}\left\langle V\left(\lambda_{i}\right), \frac{d \gamma}{d \lambda}\left(\lambda_{i}^{+}\right)-\frac{d \gamma}{d \lambda}\left(\lambda_{i}^{-}\right)\right\rangle \\
& -\left\langle V(0), \frac{d \gamma}{d \lambda}(0)\right\rangle+\left\langle V(a), \frac{d \gamma}{d \lambda}(a)\right\rangle
\end{aligned}
$$

onde $V(\lambda)$ é o campo variacional de $f e$

$$
\frac{d \gamma}{d \lambda}\left(\lambda_{i}^{+}\right)=\lim _{\lambda \rightarrow \lambda_{i}, \lambda>\lambda_{i}} \frac{d \gamma}{d \lambda}(\lambda), \quad \frac{d \gamma}{d \lambda}\left(\lambda_{i}^{-}\right)=\lim _{\lambda \rightarrow \lambda_{i}, \lambda<\lambda_{i}} \frac{d \gamma}{d \lambda}(\lambda) .
$$

\section{Demonstração}

Por definição,

$$
E(s)=\int_{0}^{a}\left\langle\frac{\partial f}{\partial \lambda}, \frac{\partial f}{\partial \lambda}\right\rangle d \lambda=\sum_{i=0}^{n} \int_{\lambda_{i}}^{\lambda_{i+1}}\left\langle\frac{\partial f}{\partial \lambda}, \frac{\partial f}{\partial \lambda}\right\rangle d \lambda
$$

usando o lema de simetria, teremos

$$
\begin{aligned}
\frac{d}{d s} \int_{\lambda_{i}}^{\lambda_{i+1}}\left\langle\frac{\partial f}{\partial \lambda}, \frac{\partial f}{\partial \lambda}\right\rangle d \lambda & =\int_{\lambda_{i}}^{\lambda_{i+1}} 2\left\langle\frac{D}{d s} \frac{\partial f}{\partial \lambda}, \frac{\partial f}{\partial \lambda}\right\rangle d \lambda \\
& =2 \int_{\lambda_{i}}^{\lambda_{i+1}}\left\langle\frac{D}{d \lambda} \frac{\partial f}{\partial s}, \frac{\partial f}{\partial \lambda}\right\rangle d \lambda \\
& =2 \int_{\lambda_{i}}^{\lambda_{i+1}} \frac{d}{d \lambda}\left\langle\frac{\partial f}{\partial s}, \frac{\partial f}{\partial \lambda}\right\rangle d \lambda-2 \int_{\lambda_{i}}^{\lambda_{i+1}}\left\langle\frac{\partial f}{\partial s}, \frac{D}{d \lambda} \frac{\partial f}{\partial \lambda}\right\rangle d \lambda .
\end{aligned}
$$

Logo, 


$$
\frac{1}{2} \frac{d E}{d s}=\left.\sum_{i=0}^{n}\left\langle\frac{\partial f}{\partial s}, \frac{\partial f}{\partial \lambda}\right\rangle\right|_{\lambda_{i}} ^{\lambda_{i+1}}-\int_{0}^{a}\left\langle\frac{\partial f}{\partial s}, \frac{D}{d \lambda} \frac{\partial f}{\partial \lambda}\right\rangle d \lambda .
$$

Observando que a soma no lado direito de (1.3) pode ser reescrita como

$$
\begin{aligned}
\left.\sum_{i=0}^{n}\left\langle\frac{\partial f}{\partial s}, \frac{\partial f}{\partial \lambda}\right\rangle\right|_{\lambda_{i}} ^{\lambda_{i+1}} & =\left.\left\langle\frac{\partial f}{\partial s}, \frac{\partial f}{\partial \lambda}\right\rangle\right|_{\lambda_{1}^{-}}-\left.\left\langle\frac{\partial f}{\partial s}, \frac{\partial f}{\partial \lambda}\right\rangle\right|_{\lambda_{0}}+\left.\left\langle\frac{\partial f}{\partial s}, \frac{\partial f}{\partial \lambda}\right\rangle\right|_{\lambda_{2}^{-}} \\
& -\left.\left\langle\frac{\partial f}{\partial s}, \frac{\partial f}{\partial \lambda}\right\rangle\right|_{\lambda_{1}^{+}}+\ldots+\left.\left\langle\frac{\partial f}{\partial s}, \frac{\partial f}{\partial \lambda}\right\rangle\right|_{\lambda_{n+1}}-\left.\left\langle\frac{\partial f}{\partial s}, \frac{\partial f}{\partial \lambda}\right\rangle\right|_{\lambda_{n}^{-}} \\
& =-\left\langle V(0), \frac{d \gamma}{d \lambda}(0)\right\rangle+\left\langle V(a), \frac{d \gamma}{d \lambda}(a)\right\rangle \\
& -\sum_{i=1}^{n}\left\langle V\left(\lambda_{i}\right), \frac{d \gamma}{d \lambda}\left(\lambda_{i}^{+}\right)-\frac{d \gamma}{d \lambda}\left(\lambda_{i}^{-}\right)\right\rangle
\end{aligned}
$$

conclui-se a demonstração.

Variações não-próprias da energia e o Princípio de Fermat. Procuraremos agora entender o comportamento, com relação a variações da energia, de geodésicas nulas ligando um evento $p$ a uma curva tipo-tempo $\eta:\left(t_{1}, t_{2}\right) \rightarrow M$ dada. Isto conduz a variações que não mantém fixos os dois extremos da curva original constituindo variações não-próprias da energia.

Por vezes, quando se deseja estudar este problema (como em [12] ou [7]), introduz-se como funcional de interesse o chamado tempo de chegada essencialmente definido pelo ítem iii) da definição 1.6. Nós, no entanto, manteremos como nosso objeto de estudo o funcional energia. Devemos notar a evidente diferença entre o caso das variações não-próprias e o das variações próprias: agora na equação para a primeira variação de energia permanece em (1.2) o termo $\left\langle V(a), \frac{d \gamma}{d \lambda}(a)\right\rangle$, onde $a=\eta(t), t \in\left(t_{1}, t_{2}\right)$.

Vamos considerar, para iniciar a discussão, o espaço de geodésicas nulas $C^{\infty}$ conectando o ponto $p$ à curva tipo-tempo $\eta$ :

Definição 1.6 O conjunto de curvas nulas conectando $p$ à curva tipo-tempo $\eta$ dada é definido como:

$\Omega_{p, \eta}:=\left\{\gamma \mid \gamma:[0, a] \stackrel{C^{\infty}}{\rightarrow} M\right.$ satisfazendo:

(i) $\left\langle\frac{d \gamma}{d \lambda}, \frac{d \gamma}{d \lambda}\right\rangle=0$;

(ii) $\gamma(0)=p$;

(iii) existe $t(\gamma) \in\left(t_{1}, t_{2}\right)$ tal que $\gamma(a)=\eta(t(\gamma))$; 


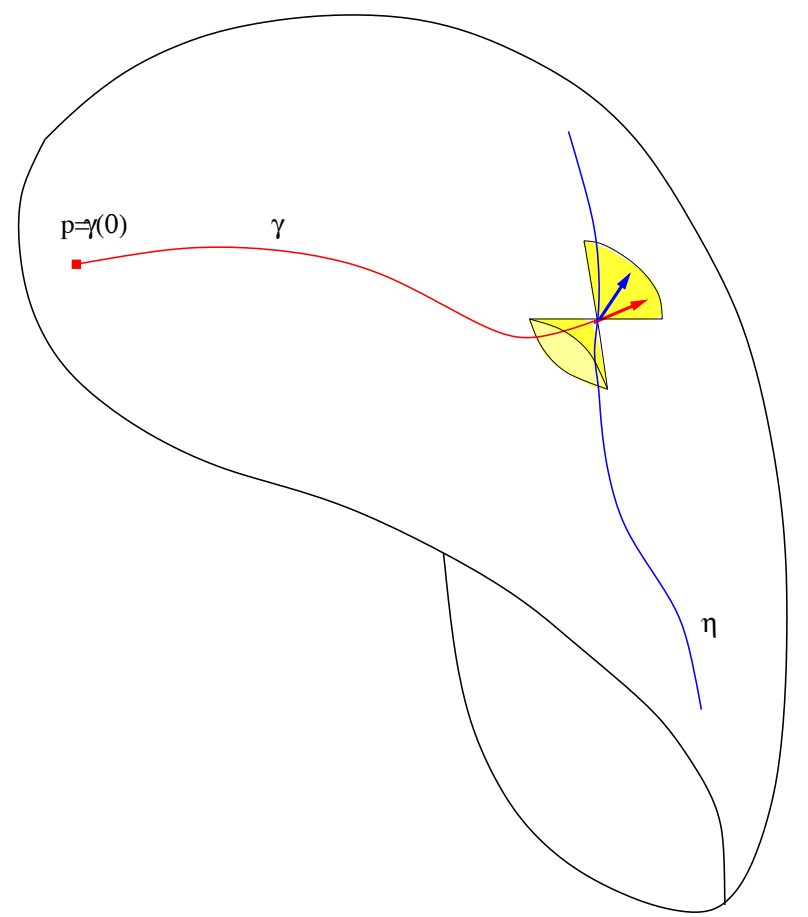

Fig. 1.2 Esquema ilustrando as curvas no conjunto $\Omega_{p, \eta}$, ou seja, curvas nulas conectando o ponto $p=\gamma(0)$ à curva $\eta$, de modo que o ponto $\gamma(a)$ esteja na curva $\eta$ e que os vetores tangentes às curvas $\eta$ e $\gamma$ no ponto $\gamma(a)$ estejam no mesmo cone tempo.

(iv) $\left.\left\langle\frac{d \gamma}{d \lambda}(a), \frac{d \eta}{d t}(t(\gamma))\right\rangle<0\right\}$.

Ou seja, neste conjunto estão curvas nulas $[(i)]$ que se iniciam em $p[(i i)]$, alcançam em tempo finito uma curva tipo-tempo dada $[(i i i)]$ e que, quando interceptam esta curva, ambas têm vetores tangentes no mesmo cone tempo do ponto de interseção, como tenta-se esquematizar na Figura 1.2.

O conjunto $\Omega_{p, \eta}$ pode ser entendido como uma variedade [20] e o espaço tangente a ela em um ponto $\gamma$ (que é um espaço vetorial de dimensão infinita) pode ser formulado como:

Definição $1.7 O$ espaço tangente a $\Omega_{p, \eta}$ em $\gamma$ é o conjunto:

$T_{\gamma} \Omega_{p, \eta}:=\left\{V:[0, a] \rightarrow T M \mid\right.$ existe uma variação $C^{\infty} f$ de $\gamma$ tal que $\left.V(\lambda)=\frac{\partial f}{\partial s}(0, \lambda), \lambda \in[0, a]\right\}$. 
Alguns lemas serão necessários para que possamos expressar o princípio de Fermat. Estes lemas fornecerão caracterizações dos elementos de $\Omega_{p, \eta}$ e de $T_{\gamma} \Omega_{p, \eta}$.

Lema 1.8 Seja $\gamma:[0, a] \rightarrow M$ um elemento de $\Omega_{p, \eta}$ e $V:[0, a] \rightarrow T M$ um campo de vetores $C^{\infty}$ ao longo de $\gamma$. São equivalentes:

(i) $V \in T_{\gamma} \Omega_{p, \eta}$;

(ii) $V$ satisfaz $V(0)=0, V(a) \| \frac{d \eta}{d t}(t(\gamma)) e\left\langle\frac{D}{d \lambda} V, \frac{d \gamma}{d \lambda}\right\rangle=0$.

\section{Demonstração}

$(i) \Rightarrow(i i)$

Como $\gamma$ é suposta ser tipo-nula,

$$
\begin{aligned}
0 & =\left.\frac{1}{2} \frac{d}{d s}\left\langle\frac{\partial f}{\partial \lambda}(s, \lambda), \frac{\partial f}{\partial \lambda}(s, \lambda)\right\rangle\right|_{s=0}=\left.\left\langle\frac{D}{d s} \frac{\partial f}{\partial \lambda}(s, \lambda), \frac{\partial f}{\partial \lambda}(s, \lambda)\right\rangle\right|_{s=0} \\
& =\left.\left\langle\frac{D}{d \lambda} \frac{\partial f}{\partial s}(s, \lambda), \frac{\partial f}{\partial \lambda}(s, \lambda)\right\rangle\right|_{s=0}
\end{aligned}
$$

e, portanto, $\left\langle\frac{D}{d \lambda} V, \frac{d \gamma}{d \lambda}\right\rangle=0$. As outras condições são diretas.

$($ ii $) \Rightarrow(i)$

A demonstração aqui apresentada é baseada em [12], mas particularizada para o caso da variedade $M$ ser globalmente hiperbólica. As propriedades relevantes de variedades globalmente hiperbólicas para o que será apresentado foram extraídas de [18], de onde a idéia geral da construção é retirada.

A idéia é construir uma variação $C^{\infty}$ de $\gamma$ dado o campo $V$ satisfazendo as hipóteses de $(i i)$.

Primeiramente notemos que se $M$ é globalmente hiperbólica podemos escrever $M=(a, b) \times S$, sendo $S$ uma superfície de Cauchy. Nestas coordenadas o elemento de linha da métrica toma a seguinte forma:

$$
(d s)^{2}=r(x, t)\left(-(d t)^{2}+\langle d x, d x\rangle_{(x, t)}^{(3)}\right)
$$

onde o sobrescrito indica que a métrica é restrita a um espaço tridimensional e o subescrito indica que a restrição depende do ponto. Como geodésicas nulas são conformalmente invariantes podemos tomar $r(t, x)=1$. Ainda, dada uma curva tipo-tempo $\eta(t)$, uma modificação da função tempo global permite que $\eta$ seja escrita na forma $\eta(t)=(t, p), p$ em uma superfície de Cauchy. 


\section{Lentes Gravitacionais: maquinaria básica}

Se o ponto $(0, q) \in M$ for dado, toda curva diferenciável $x(t)$ em $S$ conectando os pontos $p$ e $q$ tem uma extensão a uma curva nula em $M$ da forma

$$
\gamma(t)=\left(T_{x}(t), x(t)\right)
$$

e, como $\gamma$ é suposta nula, vale que

$$
\left\langle\gamma^{\prime}(t), \gamma^{\prime}(t)\right\rangle=-\left(T_{x}^{\prime}(t)\right)^{2}+\left\langle x^{\prime}(t), x^{\prime}(t)\right\rangle_{(x, t)}^{(3)}=0
$$

e $\gamma(t)$ é obtida resolvendo a equação diferencial

$$
T_{x}^{\prime}(t)=\sqrt{\left\langle x^{\prime}(t), x^{\prime}(t)\right\rangle_{(x, t)}^{(3)}} .
$$

Consideremos agora a curva $x(t)$ e, tomando as componentes $\left(V^{1}(t), V^{2}(t), V^{3}(t)\right)$ do campo $V$, definamos a variação de $x$ em $S$ como

$$
(\epsilon, t) \mapsto x^{i}(t)+\epsilon V^{i}(t) \quad i=1,2,3 .
$$

Por continuidade da solução de (1.4), para $\epsilon$ suficientemente pequenos, a variação em $x(t)$ dá origem a uma variação $C^{\infty}$ de $\gamma(t)$. Ainda a variação em $x(t)$ definida mantém o ponto final $x(a)$ fixo pois $V^{i}(a)=0$ por hipótese. Como este ponto está na curva $\eta$, que pode ser escrita na forma $\eta(t)=(t, x(a))$, temos que o ponto final da variação permanece sobre $\eta$.

Como construímos uma variação $C^{\infty}$ de $\gamma$, podemos associar a esta variação um campo variacional $\tilde{V}$. Por construção as componentes espaciais de $V$ e de $\tilde{V}$ são comuns. Entretanto vale para cada um dos campos variacionais que $\left\langle\frac{D}{d t} V, \frac{d \gamma}{d t}\right\rangle=\left\langle\frac{D}{d t} \tilde{V}, \frac{d \gamma}{d t}\right\rangle=0 \mathrm{e}$, como os campos variacionais são sujeitos às condições iniciais $V(0)=\tilde{V}(0)=0$, ambos coincidem.

Vamos agora tomar geodésicas nulas e estudar o comportamento da energia ante variações. O lema que segue mostra que geodésicas são curvas para as quais as variações no extremo do intervalo anulam-se:

Lema 1.9 Para todo elemento $\gamma:[0, a] \rightarrow M$ de $\Omega_{p, \eta}$ são equivalentes:

(i) $\gamma$ é uma geodésica;

(ii) $V(a)=0$ para todo $V \in T_{\gamma} \Omega_{p, \gamma}$.

\section{Demonstração}


$(i) \Rightarrow(i i)$

A afirmação de que $\gamma$ é uma geodésica pode ser refraseada como

$$
\frac{D}{d \lambda} \frac{d \gamma}{d \lambda}=w(\lambda) \frac{d \gamma}{d \lambda}
$$

ou seja, que seu vetor tangente é paralelamente propagado ao longo de si (embora neste caso haja uma reparametrização que faz com que a equação da geodésica tenha o lado direito igual a zero).

Tomemos $V \in T_{\gamma} \Omega_{p, \eta}$. Sabemos, pelo Lema 1.8 que $\left\langle\frac{D}{d \lambda} V, \frac{d \gamma}{d \lambda}\right\rangle=0$. Logo,

$$
\frac{d}{d \lambda}\left\langle V, \frac{d \gamma}{d \lambda}\right\rangle=\left\langle V, \frac{D}{d \lambda} \frac{d \gamma}{d \lambda}\right\rangle=w(\lambda)\left\langle V, \frac{d \gamma}{d \lambda}\right\rangle
$$

Resolvendo a EDO,

$$
\left\langle V(\lambda), \frac{d \gamma}{d \lambda}(\lambda)\right\rangle=\left\langle V(0), \frac{d \gamma}{d \lambda}(0)\right\rangle \mathrm{e}^{\int_{0}^{a} w(\tilde{\lambda}) d \tilde{\lambda}}
$$

com $\lambda \in[0, a]$. Como $V(0)=0$ a única solução para (1.6) é a trivial, o que implica que $\left\langle V(a), \frac{d \gamma}{d \lambda}(a)\right\rangle=0$ e, como $V(a)$ é um múltiplo de um vetor tipotempo (Lema 1.9) e $\frac{d \gamma}{d \lambda}(a)$ é um vetor nulo, temos necessariamente $V(a)=0$.

$($ ii $) \Rightarrow(i)$

Tomemos um campo $B \in \mathfrak{X}(M)^{9}$ ao longo de $\gamma$ que satisfaça $B(0)=B(a)=$ 0 . Seja $U$ um campo paralelamente transportado ao longo de $\gamma\left(\frac{D}{d \lambda} U=0\right)$, que satisfaça $U(a)=\frac{d}{d t} \eta(t(\gamma))$. Como $U$ é tipo-tempo, $\left\langle U, \frac{d \gamma}{d \lambda}\right\rangle$ não tem zeros. O objeto

$$
V(\lambda):=B(\lambda)-\left[\int_{0}^{\lambda} \frac{\left\langle\frac{D}{d \lambda} B, \frac{d \gamma}{d \lambda}\right\rangle}{\left\langle U, \frac{d \gamma}{d \lambda}\right\rangle}(s) d s\right] U(\lambda)
$$

define um campo vetorial $V \in T_{\gamma} \Omega_{p, \eta}$ já que automaticamente satisfaz os requerimentos do Lema 1.8. Por hipótese,

$$
0=V(a)=-\left[\int_{0}^{a} \frac{\left\langle\frac{D}{d \lambda} B, \frac{d \gamma}{d \lambda}\right\rangle}{\left\langle U, \frac{d \gamma}{d \lambda}\right\rangle}(s) d s\right] U(a)
$$

e, como $U(a) \neq 0$, a integral tem que anular-se. Como

${ }^{9}$ Conjunto dos campos vetoriais suaves definidos em $M$. 


$$
\left\langle\frac{D}{d \lambda} B, \frac{1}{\left\langle U, \frac{d \gamma}{d \lambda}\right\rangle} \frac{d \gamma}{d \lambda}\right\rangle=\frac{d}{d \lambda}\left\langle B, \frac{1}{\left\langle U, \frac{d \gamma}{d \lambda}\right\rangle} \frac{d \gamma}{d \lambda}\right\rangle-\left\langle B, \frac{D}{d \lambda}\left(\frac{1}{\left\langle U, \frac{d \gamma}{d \lambda}\right\rangle} \frac{d \gamma}{d \lambda}\right)\right\rangle
$$

e $B(0)=B(a)=0$, temos que a equação (1.9) é verificada se a condição

$$
\frac{D}{d \lambda}\left(\frac{1}{\left\langle U, \frac{d \gamma}{d \lambda}\right\rangle} \frac{d \gamma}{d \lambda}\right)=0
$$

for satisfeita, ou seja, se $\frac{D}{d \lambda} \frac{d \gamma}{d \lambda}=0$.

\subsubsection{Princípio de Fermat}

Concluímos esta seção coletando as conclusões dos Lemas 1.8 e 1.9 e estabelecendo o teorema:

Teorema 1.10. (Princípio de Fermat) Uma curva $\gamma \in \Omega_{p, \eta}$ é uma geodésica, então é é estacionária. A recíproca é verdadeira para variações próprias.

Ou seja, a cada ponto estacionário de $E$ está associada uma geodésica nula ligando $p$ a $\eta$.

\subsection{Caráter dos pontos estacionários}

O Teorema 1.10 garante que curvas em $\Omega_{p, \eta}$ são geodésicas se e somente se forem estacionárias, mas não diz nada sobre a característica do extremo de $E$ associado a estas geodésicas. Podemos estudar os efeitos de uma segunda variação da energia em torno de curvas estacionárias para entender o caráter destes extremos. Estabeleceremos que os pontos estacionários de $\Omega_{p, \eta}$ são mínimos ou pontos de sela do funcional energia.

\subsubsection{Segunda variação da energia}

Restrita a geodésicas de $\Omega_{p, \eta}$, a fórmula da primeira variação da energia escrevese como:

$$
\frac{1}{2} E^{\prime}(0)=-\int_{0}^{a}\left\langle V(\lambda), \frac{D}{d \lambda} \frac{d \gamma}{d \lambda}\right\rangle d \lambda
$$

Podemos, então, estabelecer o resultado para a segunda variação da energia: 
Proposição 1.11 Sejam $\gamma:[0, a] \rightarrow M, \gamma \in \Omega_{p, \eta}$ uma geodésica e $f:(-\epsilon, \epsilon) \times$ $[0, a] \rightarrow M$ uma variação de $\gamma$ tal que o campo variacional a ela associado seja elemento de $T_{\gamma} \Omega_{p, \eta}$. Então,

$$
\frac{1}{2} E^{\prime \prime}(0)=-\int_{0}^{a}\left\langle V(\lambda), \frac{D^{2} V}{d \lambda^{2}}+R\left(\frac{d \gamma}{d \lambda}, V\right) \frac{d \gamma}{d \lambda}\right\rangle d \lambda
$$

\section{Demonstração}

Tomando a derivada de (1.11), obtemos

$$
\frac{1}{2} \frac{d^{2} E}{d s^{2}}=-\int_{0}^{a}\left\langle\frac{D}{d s} \frac{\partial f}{\partial s}, \frac{D}{d \lambda} \frac{\partial f}{\partial \lambda}\right\rangle d \lambda-\int_{0}^{a}\left\langle\frac{\partial f}{\partial s}, \frac{D}{d s} \frac{D}{d \lambda} \frac{\partial f}{\partial \lambda}\right\rangle d \lambda
$$

fazendo $s=0$ temos que o primeiro termo no lado direito da expressão acima anula-se pois $\gamma$ é, por hipótese, uma geodésica. Além disso, como

$$
\frac{D}{d s} \frac{D}{d \lambda} \frac{\partial f}{\partial \lambda}=\frac{D}{d \lambda} \frac{D}{d s} \frac{\partial f}{\partial \lambda}+R\left(\frac{\partial f}{\partial \lambda}, \frac{\partial f}{\partial s}\right) \frac{\partial f}{\partial \lambda},
$$

temos, em $s=0$

$$
\frac{D}{d s} \frac{D}{d \lambda} \frac{\partial f}{\partial \lambda}=\frac{D^{2}}{d \lambda^{2}} V+R\left(\frac{d \gamma}{d \lambda}, V\right) \frac{d \gamma}{d \lambda}
$$

e portanto obtém-se o enunciado.

Consideremos (1.12). Como

$$
\frac{d}{d \lambda}\left\langle V, \frac{D V}{d \lambda}\right\rangle=\left\langle V, \frac{D^{2} V}{d \lambda^{2}}\right\rangle+\left\langle\frac{D V}{d \lambda}, \frac{D V}{d \lambda}\right\rangle,
$$

podemos escrever

$$
\begin{aligned}
\int_{0}^{a} & \left\langle V(\lambda), \frac{D^{2} V}{d \lambda^{2}}+R\left(\frac{d \gamma}{d \lambda}, V\right) \frac{d \gamma}{d \lambda}\right\rangle d \lambda \\
= & \int_{0}^{a} \frac{d}{d \lambda}\left\langle V, \frac{D V}{d \lambda}\right\rangle d \lambda-\int_{0}^{a}\left(\left\langle\frac{D V}{d \lambda}, \frac{D V}{d \lambda}\right\rangle-\left\langle V, R\left(\frac{d \gamma}{d \lambda}, V\right) \frac{d \gamma}{d \lambda}\right\rangle\right) d \lambda \\
= & \left.\left\langle V, \frac{D V}{d \lambda}\right\rangle\right|_{0} ^{a}-\int_{0}^{a}\left(\left\langle\frac{D V}{d \lambda}, \frac{D V}{d \lambda}\right\rangle-\left\langle V, R\left(\frac{d \gamma}{d \lambda}, V\right) \frac{d \gamma}{d \lambda}\right\rangle\right) d \lambda \\
= & -\int_{0}^{a}\left(\left\langle\frac{D V}{d \lambda}, \frac{D V}{d \lambda}\right\rangle-\left\langle R\left(\frac{d \gamma}{d \lambda}, V\right) \frac{d \gamma}{d \lambda}, V\right\rangle\right) d \lambda
\end{aligned}
$$

Motivados por este desenvolvimento, introduzimos o seguinte objeto: 
18 Lentes Gravitacionais: maquinaria básica

Definição 1.12 Seja $\gamma \in \Omega_{p, \eta}$ uma geodésica. Dados $A, B \in T_{\gamma} \Omega_{p, \eta}$ definimos a Forma do Índice associada a $\gamma$ como:

$$
I_{\gamma}(A, B)=\int_{0}^{a}\left(\left\langle\frac{D A}{d \lambda}, \frac{D B}{d \lambda}\right\rangle-\left\langle R\left(\frac{d \gamma}{d \lambda}, A\right) \frac{d \gamma}{d \lambda}, B\right\rangle\right) d \lambda
$$

$R(.$, . ) . é o tensor de Riemann. Notamos que a forma do índice é uma forma bilinear simétrica associada a uma forma quadrática em $T_{\gamma} \Omega_{p, \eta}$. Esta forma pode ser entendida como o hessiano do funcional energia $E: \Omega_{p, \eta} \rightarrow \mathbb{R}$ em pontos críticos. De fato, comparando-se (1.12) e (1.15) vê-se que:

Lema 1.13 Sejam $\gamma:[0, a] \rightarrow M$ uma geodésica em $\Omega_{p, \eta}$. Para $V$ um campo variacional em $T_{\gamma} \Omega_{p, \eta}$,

$$
E^{\prime \prime}(0)=2 I_{\gamma}(V, V)
$$

Encontraremos pela primeira vez na discussão do caráter dos pontos críticos do funcional energia o conceito de pontos conjugados. De fato, demonstra-se também para o caso Lorentziano um resultado que em geometria Riemanniana é conhecido como Lema do Índice [9]:

Lema 1.14 Seja $\gamma \in \Omega_{p, \eta}$ uma geodésica sem pontos conjugados. Então todo campo variacional não trivial $V \in T_{\gamma} \Omega_{p, \eta}$ satisfaz $I_{\gamma}(V, V)>0$.

A definição de ponto conjugado é apresentada no Apêndice A.7, mas pode ser entendido como um ponto ao longo de uma geodésica no qual geodésicas arbitrariamente próximas com ponto inicial comum, mas com uma velocidade inicial de separação positiva, voltam a cruzar-se por conta dos efeitos da curvatura. A caracterização destes pontos é feita através do estudo dos campos de Jacobi, assunto que nos será recorrente e, apesar de também descrito no Apêndice A.7, merece ser recobrado já que, como se vê em (1.12), $I_{\gamma}=0$ se a variação satisfizer a equação de Jacobi, ou seja, se

$$
\frac{D^{2} V}{d \lambda^{2}}+R\left(\frac{d \gamma}{d \lambda}, V\right) \frac{d \gamma}{d \lambda}=0
$$

Sabemos que variações devem anular-se em $p$, ou seja, $V(0)=0$ e $V^{\prime}(0)=V_{0}$. Os pontos conjugados ao longo de $\gamma$ correspondem a pontos nos quais $V$ volta a anular-se, supondo que não seja identicamente nulo. De maneira esquemática, 


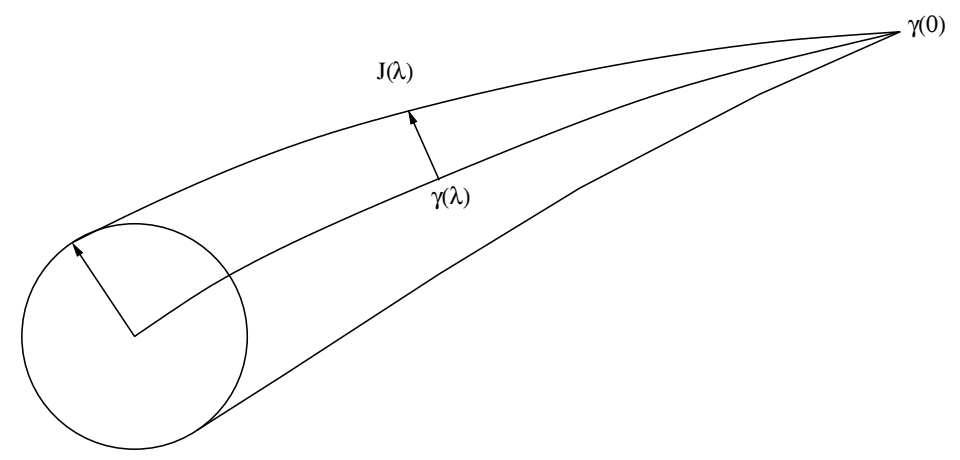

Fig. 1.3 Campo de Jacobi ao longo de $\gamma$. O campo de Jacobi descreve como geodésicas arbitrariamente próximas afastam-se sob efeito da curvatura do espaço-tempo.

campos de Jacobi podem ser vistos como um vetor com origem em $\gamma$ e cuja "ponta" traça geodésicas nulas vizinhas a $\gamma$. É importante ressaltar que em geral $J \notin T_{p} \Omega_{p, \eta}$ por não necessariamente satisfazer as condições de contorno para os campos de variação em $\gamma(a)$.

Se um ponto $\gamma(s)$ é conjugado a $\gamma(0)$, então há um feixe de geodésicas nulas emergindo de $\gamma(0)$ e que são refocadas em $\gamma(s)$ dentro da aproximação linear.

Dada sua ligação com campos de Jacobi e a interpretação destes últimos ser tão ligada à construção do problema de lentes, vemos que a forma do índice é central no estudo de lentes gravitacionais, o que se pode confirmar pelo enunciado do teorema que segue:

Teorema 1.15 Seja $\gamma \in \Omega_{p, \eta}$ uma geodésica. Então,

(i) Se $\gamma$ é livre de pontos conjugados, $\gamma$ é um minimo local da função energia, isto é, para qualquer $V \in T_{\gamma} \Omega_{p, \eta}$ não trivial, $I_{\gamma}(V, V)>0$.

(ii) Se $\gamma$ passa por um ponto conjugado antes de alcançar $\eta, \gamma$ é um ponto de sela do funcional energia.

\section{Demonstração}

(i) Consequência direta dos Lemas 1.13 e 1.14.

(ii) Consideremos válido o seguinte lema:

Lema 1.16 Seja $\gamma:[0, a] \rightarrow M$ uma geodésica elemento de $\Omega_{p, \eta}$. São equivalentes as condições:

(i) $V \in T_{\gamma} \Omega_{p, \eta}$; 
20 Lentes Gravitacionais: maquinaria básica

(ii) $V(0)=V(a)=0$ e $\left\langle V, \frac{d \gamma}{d \lambda}\right\rangle=0$.

Seja $\lambda_{c}$ o parâmetro do primeiro ponto conjugado a 0 ao longo de $\gamma$. Escolhemos um campo de vetores $C^{\infty} V$ ao longo de $\gamma$ tal que $\left\langle V, \frac{d \gamma}{d \lambda}\right\rangle=0$, $V(0)=0$ e $V(\lambda)=0$ para $\lambda \in\left[\lambda_{c}, a\right]$. Pelo Lema 1.16, $V$ é elemento de $T_{\lambda} \Omega_{p, \eta}$. Se $V$ não é em todo ponto paralelo a $\frac{d \gamma}{d \lambda}$ então, pelo Lema $1.14, I_{\gamma}(V, V)>0$ e, portanto, pelo Lema 1.13 dá origem a curvas com valores maiores da função energia.

Por outro lado, podemos construir uma curva vizinha a $\gamma$ à qual corresponde um valor menor da energia pelo seguinte método: Por hipótese $\lambda_{c}$ é conjugado a 0 , o que significa que existe um campo de Jacobi $J$ não trivial ao longo de $\gamma$ (nem paralelo a $\frac{d \gamma}{d \lambda}$ ) que satisfaz $J(0)=J\left(\lambda_{c}\right)=0$. Naturalmente $J\left(\lambda_{c}\right)$ não é paralelo a $\frac{d \gamma}{d \lambda}\left(\lambda_{c}\right)$ e satisfaz $\left\langle J, \frac{d \gamma}{d \lambda}\right\rangle=0\left(\mathrm{e}\left\langle\frac{D}{d \lambda} J, \frac{d \gamma}{d \lambda}\right\rangle=0\right.$, ou seja, $\frac{D J}{d \lambda}$ é tipo-espaço). Podemos definir um campo $Z$ ao longo de $\gamma$ por $\frac{D}{d \lambda} Z=0$ e $Z\left(\lambda_{c}\right)=-\frac{D}{d \lambda} J\left(\lambda_{c}\right)$. Para todo $\alpha \in \mathbb{R}$,

$$
A_{\alpha}(\lambda):= \begin{cases}J(\lambda)+\alpha \frac{\lambda}{\lambda_{c}} Z(\lambda) & \text { para } \lambda \in\left[0, \lambda_{c}\right] \\ \alpha \frac{a-\lambda}{a-\lambda_{c}} Z(\lambda) & \text { para } \lambda \in\left(\lambda_{c}, a\right] ;\end{cases}
$$

define um campo de vetores contínuo ao longo de $\gamma, C^{\infty}$ exceto em $\lambda=\lambda_{c}$, que satisfaz $\left\langle A_{\alpha}, \frac{d \gamma}{d \lambda}\right\rangle=0 \operatorname{com} A_{\alpha}(0)=A_{\alpha}(a)=0$. Como todos os termos que restam no cálculo de $I_{\gamma}\left(A_{\alpha}, A_{\alpha}\right)$ são proporcionais a $\alpha$, vale (convém lembrar de (1.12) e que $J$ é campo de Jacobi):

$$
\left.I_{\gamma}\left(A_{\alpha}, A_{\alpha}\right)\right|_{\alpha=0}=0
$$

O termo linear em $\alpha$ em $I_{\gamma}\left(A_{\alpha}, A_{\alpha}\right)$ pode ser expresso como 


$$
\begin{aligned}
-\alpha \int_{0}^{\lambda_{c}}\left(\frac{\lambda}{\lambda_{c}}\right)\left\langle R\left(\frac{d \gamma}{d \lambda}, Z\right) \frac{d \gamma}{d \lambda}, J\right\rangle d \lambda & =-\alpha \int_{0}^{\lambda_{c}}\left(\frac{\lambda}{\lambda_{c}}\right)\left\langle R\left(\frac{d \gamma}{d \lambda}, J\right) \frac{d \gamma}{d \lambda}, Z\right\rangle d \lambda \\
& =\alpha \int_{0}^{\lambda_{c}}\left(\frac{\lambda}{\lambda_{c}}\right)\left\langle\frac{D^{2} J}{d \lambda^{2}}, Z\right\rangle d \lambda \\
& =\alpha \int_{0}^{\lambda_{c}} \frac{d}{d \lambda}\left\langle\frac{D J}{d \lambda},\left(\frac{\lambda}{\lambda_{c}}\right) Z\right\rangle d \lambda \\
& -\alpha \int_{0}^{\lambda_{c}} \frac{d}{d \lambda}\left\langle\frac{D J}{d \lambda}, \frac{D}{d \lambda}\left(\frac{\lambda}{\lambda_{c}}\right) Z\right\rangle d \lambda \\
& =\alpha\left[\left\langle\frac{D J}{d \lambda},\left(\frac{\lambda}{\lambda_{c}}\right) Z\right\rangle+\left\langle J,\left(\frac{1}{\lambda_{c}}\right) Z\right\rangle_{0}^{\lambda_{c}}\right. \\
& =-\alpha\left\langle\frac{D J}{d \lambda}\left(\lambda_{c}\right), \frac{D J}{d \lambda}\left(\lambda_{c}\right)\right\rangle
\end{aligned}
$$

utilizando propriedades da curvatura, campos de Jacobi e hipóteses do teorema. Como $\frac{D}{d \lambda} J$ é tipo-espaço,

$$
\left.\frac{d}{d \alpha} I_{\gamma}\left(A_{\alpha}, A_{\alpha}\right)\right|_{\alpha=0}=-2\left\langle\frac{D}{d \lambda} J\left(\lambda_{c}\right), \frac{D}{d \lambda} J\left(\lambda_{c}\right)\right\rangle<0 .
$$

Portanto $\left.I_{\gamma}\left(A_{\alpha}, A_{\alpha}\right)\right|_{\alpha=0}<0$ para algum $\alpha$ suficientemente pequeno. Se $A_{\alpha}$ é suavizado apropriadamente em torno $\lambda_{c}$, obtemos um campo variacional $A \in$ $T_{\lambda} \Omega_{p, \eta}$ que conduz a curvas com valores menores de energia que $\gamma$.

\section{Demonstração (Lema 1.16)}

$(i) \Rightarrow($ ii $)$ Pelo Lema 1.8, $V$ satisfaz $V(0)=0$ e $\left\langle\frac{D}{d \lambda} V, \frac{d \gamma}{d \lambda}\right\rangle=0$. Como $\frac{D^{2}}{d \lambda^{2}} \gamma=0,\left\langle V, \frac{d \gamma}{d \lambda}\right\rangle$ é constante e assim $\left\langle V, \frac{d \gamma}{d \lambda}\right\rangle=0$ já que $V(0)=0 . V(a)=0$ pois $\gamma$ é estacionária.

$(i i) \Rightarrow(i)$ Como $\frac{D^{2}}{d \lambda^{2}} \gamma=0$ e $\left\langle V, \frac{d \gamma}{d \lambda}\right\rangle=0$, temos $\left\langle\frac{D}{d \lambda} V, \frac{d \gamma}{d \lambda}\right\rangle=0$. Pelo Lema 1.8, $V \in T_{\gamma} \Omega_{p, \eta}$.

Esta caracterização dos extremos do funcional energia, juntamente com o princípio de Fermat permite a introdução do que se chama potencial de Fermat [21] no estudo de lentes gravitacionais. Este potencial, no estudo de lentes finas, é caracterizado, além das propriedades da distribuição de matéria, pela posição da fonte e da imagem (relacionadas por meio de um parâmetro de impacto e um ângulo de deflexão) e denotado por $\phi(\mathbf{x}, \mathbf{y})$, sendo o primeiro argumento a posição da imagem e o segundo a posição da fonte. O princípio de Fermat implica que a posição da imagem (ou das imagens) será dada pelos x's que fazem com que $\nabla \phi(\mathbf{x}, \mathbf{y})=0$, como descrito em [21].

Deve-se notar que, como mostrado, existe pelo menos um mínimo associado 
22 Lentes Gravitacionais: maquinaria básica

ao potencial de Fermat, ou seja, para qualquer posicionamento da fonte em relação à lente existe sempre pelo menos uma imagem sua . Além do potencial crescer sempre que afastamos $\mathbf{x}$ do valor associado ao mínimo do potencial, os extremos do potencial de Fermat - que estão associados aos extremos da energia - são sempre mínimos ou pontos de sela. Estes dois fatos conjuntos indicam uma aproximação ao argumento de que sempre existe um número ímpar de imagens associados a uma configuração de lentes (supondo o defletor transparente), embora a construção rigorosa do argumento seja bastante mais elaborada e formulada em termos de elementos que mencionaremos na Seção 1.4 .

Muito embora não possamos estabelecer o resultado que afirma que sempre há um número ímpar de imagens em problemas de lentes gravitacionais (observadas certas condições), mostraremos um resultado bastante mais simples mas bastante esclarecedor do papel de pontos conjugados no tratamento de lentes gravitacionais, a saber, mostraremos que a existência de pontos conjugados ao longo de $\gamma$ é suficiente para a presença de imagens múltiplas.

\subsection{Pontos conjugados e imagens múltiplas}

Veremos que uma condição suficiente para a existência de imagens múltiplas nos espaços-tempo que tomamos em consideração é que haja pelo menos um ponto conjugado ao longo da geodésica conectando fonte e observador ${ }^{10}$. Para tanto iniciaremos retomando duas proposições de [15], a saber as proposições 4.5.10 e 4.5.12:

Duas proposições de [15].

Proposição 1.17. (Proposição 4.5.10 de [15]) Se p e q são unidos por uma curva não-tipo-espaço $\gamma(\lambda)$ que não é uma geodésica nula, então eles também podem ser unidos por uma curva tipo-tempo.

Proposição 1.18. (Proposição 4.5.12 de [15]) Se há um ponto $r$ em $(p, q)$ conjugado a q ao longo de $\gamma(\lambda)$ então há uma variação de $\gamma(\lambda)$ que dá origem a uma curva tipo-tempo de $q$ até $p$.

\footnotetext{
${ }^{10}$ Legaremos a uma discussão futura (seção 2.23) as condições - bastante abrangentes - sobre as quais pode-se afirmar a existência de pontos conjugados ao longo de geodésicas.
} 

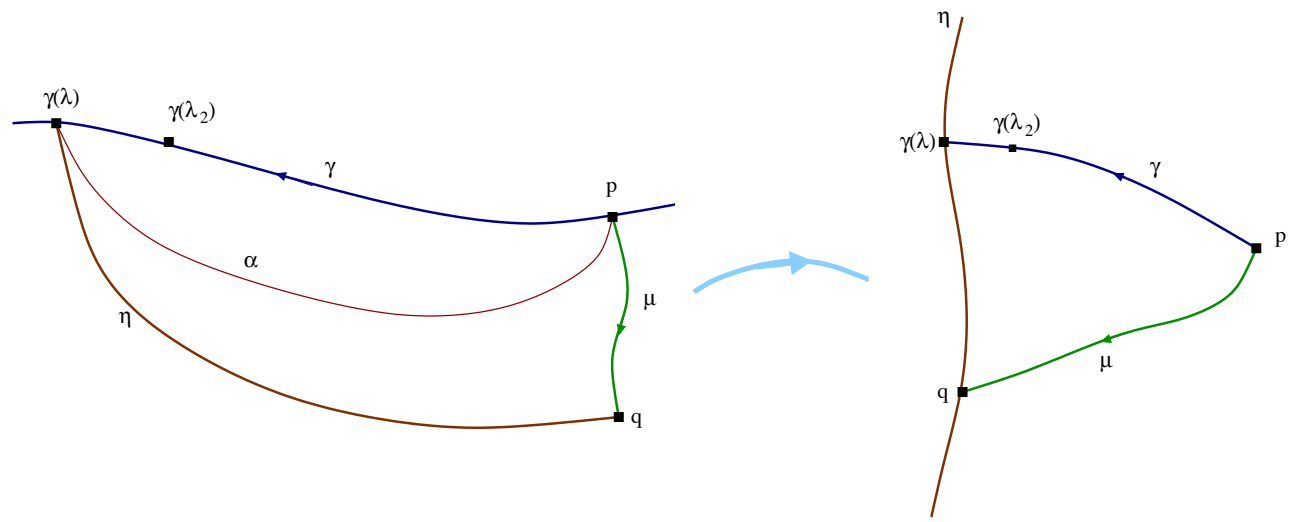

Fig. 1.4 Esquematização da construção utilizada na demonstração do Teorema 1.19 e reestruturação para que se aproxime mais do usual para uma configuração de lentes. $\gamma(\lambda)$ designa um ponto conjugado a $\gamma\left(\lambda_{1}\right)$ ao longo da geodésica nula $\gamma$.

Esta última proposição afirma que um observador movendo-se a uma velocidade subluminal pode alcançar um raio de luz $\gamma$ após este ter passado por um ponto conjugado.

Dois teoremas de [22]. Enunciaremos agora os teoremas centrais na conexão entre existência de pontos conjugados ao longo de geodésicas nulas e existência de imagens múltiplas:

Teorema 1.19 Fixemos um ponto $p \in M$. Seja $\gamma: I \rightarrow M$ uma geodésica nula direcionada para o passado com $\gamma\left(\lambda_{1}\right)=p$ e assumamos que existe um $\lambda_{2}>\lambda_{1}$ em I tal que $\gamma\left(\lambda_{2}\right)$ é conjugado a $\gamma\left(\lambda_{1}\right)$ ao longo de $\gamma$. Então através de cada ponto $\gamma(\lambda)$ com $\lambda>\lambda_{2}$ existe uma curva tipo-tempo $\eta$ que pode ser alcançada a partir de p por uma segunda geodésica nula direcionada para o passado $\mu$.

Demonstração Por hipótese, $\gamma\left(\lambda_{2}\right)$ é conjugado a $\gamma\left(\lambda_{1}\right)$ ao longo de $\gamma$. Assim, a Proposição 4.5.12 de [15] (transcrita acima) garante que, para qualquer $\lambda \in I \operatorname{com} \lambda>\lambda_{2}$, o ponto $\gamma(\lambda)$ pode ser unido a $p$ por uma curva tipo-tempo $\alpha$. Por continuidade, $\gamma(\lambda)$ pode ser conectado a qualquer ponto $q$ em uma vizinhança arbitrariamente pequena de $p$ por uma curva tipo-tempo $\eta$. Claramente $q$ pode ser escolhido de modo que possa ser alcançado a partir de $p$ ao longo de uma geodésica nula direcionada para o passado (arbitrariamente curta) $\mu$. 
24 Lentes Gravitacionais: maquinaria básica

Como notado em [22], no Teorema 1.19 o ponto $p$ é dado, mas a curva $\eta$, não. Como em geral não se pode esperar que fontes de luz reais (como quasares) movam-se ao longo das linhas de mundo construídas na demonstração do teorema, é desejável um resultado semelhante no qual tanto o ponto $p$ quanto a curva $\eta$ sejam dadas. Isto é possível se uma condição adicional for imposta:

Teorema 1.20 Fixemos um ponto $p \in M$ e uma curva tipo-tempo $\eta$ que tem interseção com $J^{-}(p) \backslash I^{-}(p)$ em um ponto q diferente de $p$. Vamos assumir que $\gamma: I \rightarrow M$ é uma geodésica nula direcionada para o passado com $\gamma\left(\lambda_{1}\right)=p$, $\gamma\left(\lambda_{3}\right) \in \operatorname{Im}(\eta)$ e há um ponto $\gamma\left(\lambda_{2}\right)$ conjugado a $\gamma\left(\lambda_{1}\right)$ ao longo de $\gamma$ com $\lambda_{1}<\lambda_{2}<\lambda_{3}$. Então há uma segunda geodésica nula direcionada para o passado $\mu$ de $p$ a $\eta$.

Demonstração Como $q$ está em $J^{-}(p)$ mas não em $I^{-}(p)$, há uma curva causal direcionada para o passado $\mu$ de $p$ a $q$, mas não há uma tal curva que seja tipo-tempo. As proposições 4.5.10 e 4.5.12 de [15] (acima transcritas) garantem que $\mu$ é uma geodésica nula que não tem em seu interior pontos conjugados a $p$. Claramente $\gamma$ e $\mu$ são geodésicas diferentes já que $\gamma$ tem em seu interior um ponto conjugado a $p$.

Em contextos gerais, a condição de interseção de $\gamma$ e $J^{-}(p) \backslash I^{-}(p)$ pode ser bastante restritiva. Um exemplo dramático é o Universo de Gödel para o qual $J^{-}(p) \backslash I^{-}(p)$ é vazio [22]. Em espaços globalmente hiperbólicos, entretanto, toda curva tipo-tempo inextensível que entra em $I^{-}(p)$ tem interseção com $J^{-}(p) \backslash I^{-}(p)$ e, portanto, esta condição do teorema é automaticamente satisfeita.

Ficam, portanto, estabelecidas condições sob as quais pode-se garantir existência de imagens múltiplas. Poderia-se perguntar se estas condições são também necessárias, isto é, se é correto afirmar que dada uma configuração de imagens múltiplas corresponde a existência de pontos conjugados ao longo de uma das geodésicas nulas conectando fonte a observador. A resposta geral é não. A existência de pontos conjugados ao longo de uma geodésica está associada a interseções de geodésicas infinitesimalmente próximas. Para responder sobre interseções de geodésicas de maneira geral, deve-se estudar a existência de pontos de corte.

Para ilustrar a situação em que podem surgir imagens múltiplas na ausência de pontos conjugados ao longo de nenhuma geodésica nula ligando fonte e observador, consideremos o exemplo de uma corda cósmica de Nambu-Goto 
sobre um espaço-tempo de Minkowski [23, 24], caracterizado pelo tensor de energia momento:

$$
\left(T_{\beta}^{\alpha}\right)=\mu \delta(x) \delta(y) \operatorname{diag}(1,0,0,1)
$$

com $\mu$ sendo a tensão da corda ${ }^{11}$

Como mostrado em [26], este espaço-tempo é equivalente a um espaço de Minkowski com um déficit angular determinado pelas propriedades da corda. Neste espaço-tempo, no entanto, para certos arranjos relativos de fonte-cordaobservador, há imagens múltiplas. O valor da tensão na corda restringe o déficit angular e consequentemente os intervalos em que as imagens múltiplas são geradas. A curvatura de Riemann deste espaço, no entanto, é identicamente nula, não havendo pontos conjugados ao longo de nenhuma geodésica nula conectando fonte e observador. O estudo geral das condições necessárias para a existência de imagens múltiplas passa, portanto, pelo estudo da existência de pontos de corte $^{12}$ ao longo de geodésicas nulas no espaço-tempo [22].

Em tempo, notamos que apesar do espaço-tempo da corda apresentar apenas pontos de corte e não pontos conjugados, espaços-tempo nos quais pontos conjugados possam se dar não estão excluídos de terem também pontos de corte. No caso em que pontos conjugados e pontos de corte ocorrem, o ponto de corte sempre antecede ou coincide com o ponto conjugado [9].

Podemos fazer um breve estudo geométrico do cone de luz passado de um evento com base nesses conceitos. Rementendo-nos à Seção A.6 para a definição e propriedades do mapa exponencial, consideramos o seguinte subconjunto de $T_{p} M$ :

$$
\mathcal{C}_{p}^{-}=\left\{X \in \mathcal{W}_{p} \backslash\{0\} \mid X \text { é tipo-nulo e aponta para o passado }\right\},
$$

onde $\mathcal{W}_{p}$ denota o máximo domínio de definição de $\exp _{p}$. Denotaremos por

$$
e_{p}^{-}=\left.\exp _{p}\right|_{\mathcal{C}_{p}^{-}}: \mathcal{C}_{p}^{-} \rightarrow M
$$

a restrição do mapa exponencial ao conjunto $\mathcal{C}_{p}^{-}$. Seguindo [7], definimos o cone de luz passado de $p$ como a imagem de $e_{p}^{-}$.

${ }^{11}$ Muito embora tensores energia-momento com forma de distribuições sejam particularmente problemáticos em relatividade geral [25].

${ }^{12}$ Pontos de corte são essencialmente análogos a pontos conjugados, entretanto não são determinados pela interseção de curvas variacionalmente vizinhas, mas sim por todas as curvas no espaço-tempo conectando dois pontos de interesse. Os pontos de corte são determinados pelo estudo da distância Lorentziana entre os dois pontos [9]. 


\section{Lentes Gravitacionais: maquinaria básica}

Como sabemos o mapa exponencial sempre é um difeomorfismo se restrito a uma vizinhança suficientemente pequena $\mathcal{U}$ da origem de $T_{p} M$, ou seja, pelo menos nesta vizinhança o cone de luz é uma variedade mergulhada e portanto nela não têm lugar fenômenos críticos. Ampliando-se o domínio de definição, entretanto, o mapa exponencial pode deixar de ser injetivo e podem formar-se cáusticas, auto-interseções e outros tipos de singularidades ${ }^{13}$. É inclusive em termos das características do cone de luz que se pode, em última instância, classificar um efeito de lente gravitacional como lente fraca ou lente forte: caso o cone de luz passado seja uma subvariedade mergulhada no espaço-tempo, todo efeito de distorção gravitacional de imagens estará na classe das lentes fracas; caso falhe em ser subvariedade mergulhada, temos uma lente forte. Devemos ainda destacar que esta classificação depende do domínio de definição das geodésicas nulas que formam o cone, já que em uma grande classe de espaços-tempo em Relatividade Geral, geodésicas arbitrariamente prolongáveis encontram pontos conjugados.

Consideremos o que acontece quando geodésicas nulas encontram pontos conjugados: como dissemos, a diferencial do mapa exponencial tem posto máximo se restrita a vetores $X \in \mathcal{C}_{p}^{-} \cap \mathcal{U}$ e daí $e_{p}^{-}$é uma subvariedade (de dimensão 3) imersa em $M$. Sabemos também que o mapa exponencial deixa de ser difeomorfismo quando pontos conjugados são alcançados, e nesses pontos o posto de sua diferencial tem que ser reduzido da multiplicidade do ponto conjugado (que, como veremos pode ser 1 ou 2) e portanto $e_{p}^{-}$deixa de ser uma subvariedade imersa. Este fato geométrico sobre o cone de luz é consoante ao fato já demonstrado que pontos conjugados são suficientes para a formação de imagens múltiplas. Em geral emprega-se a denominação de cáustica do cone de luz passado de $p$ ao conjunto de pontos conjugados a $p$ ao longo de geodésicas nulas emanando deste ponto. O dramático exemplo da lente produzida pela corda cósmica mostra que o cone de luz passado de $p$ pode deixar de ser uma variedade mergulhada ainda que a cáustica de $p$ seja vazia, ou seja, que não hajam pontos conjugados e portanto que a diferencial do mapa exponencial seja injetiva. Este é o caso, por exemplo, em que há auto interseções no cone de luz passado tais que a diferencial do mapa exponencial não se anula, mas o mapa exponencial deixa de ser invertível em sua imagem [7].

${ }^{13}$ Ver [27] para uma discussão dos tipos de singularidades possíveis através de um formalismo baseado em variedades de Lagrange. 


\subsection{Lentes gravitacionais, pontos críticos e topologia}

Introduziremos nesta seção alguns elementos que permitam ilustrar a maneira pela qual demonstram-se um dos resultados formais mais importantes da teoria das lentes gravitacionais, a saber, a afirmação de que há sempre um número ímpar de imagens em um sistema de lente gravitacional que tenha um defletor transparente [28]. Embora não demonstremos este teorema e, em verdade, nem sequer o enunciemos, ilustraremos alguns conceitos da teoria de Morse em um exemplo simples (e sem muita precisão), enunciaremos as desigualdades de Morse e em seguida teceremos alguns comentários sobre a transposição destas estruturas para o caso de interesse nos sistemas de lentes gravitacionais.

\subsubsection{Ilustração da teoria de Morse}

Consideremos o exemplo fornecido na introdução de [29] como ilustração da conexão entre topologia de uma variedade e conjunto de pontos críticos de uma função sobre ela definida: Tomemos um toro $M$ tangente ao plano $V$ no ponto $p$ como indicado na figura:

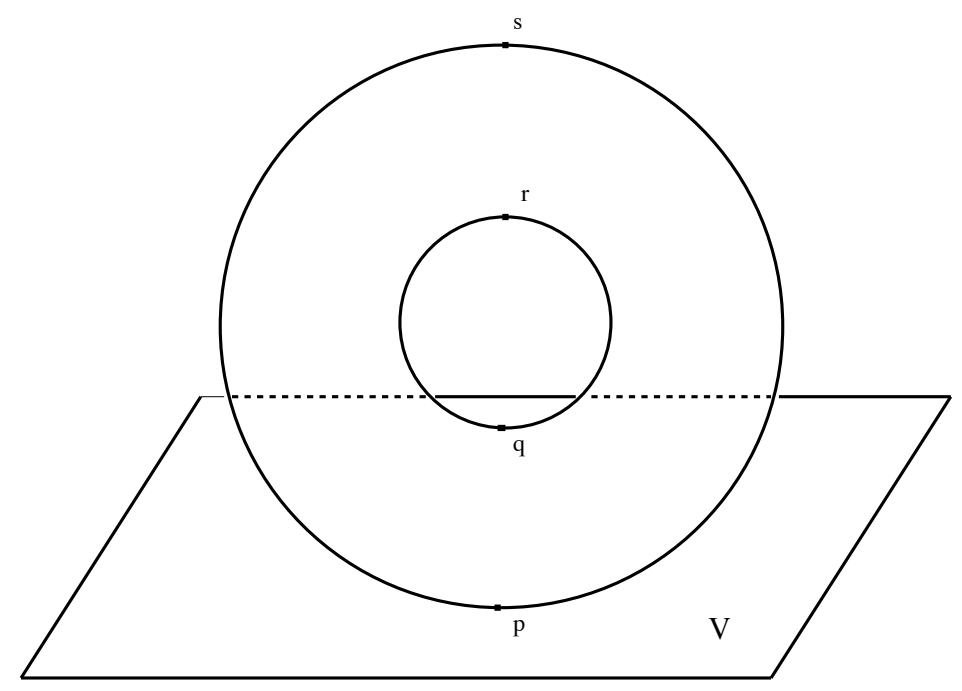

Fig. 1.5 Figura básica para a ilustração da conexão entre topologia de uma variedade e o conjunto de pontos críticos de uma certa função nela definida. Neste caso a função estudada é a altura de pontos com relação ao plano $V$ e os pontos críticos são $p, q, r$ e $s$. 
28 Lentes Gravitacionais: maquinaria básica

Seja $f: M \rightarrow \mathbb{R}$ a altura dos pontos de $M$ com relação ao plano $V$ e seja $M^{a}$ o conjunto de pontos $x \in M$ tais que $f(x) \leq a$. Então,

(i) Se $a<0<f(p)$, então $M^{a}$ é vazio;

(ii) Se $f(p)<a<f(q)$, então $M^{a}$ tem a forma de uma canoa:

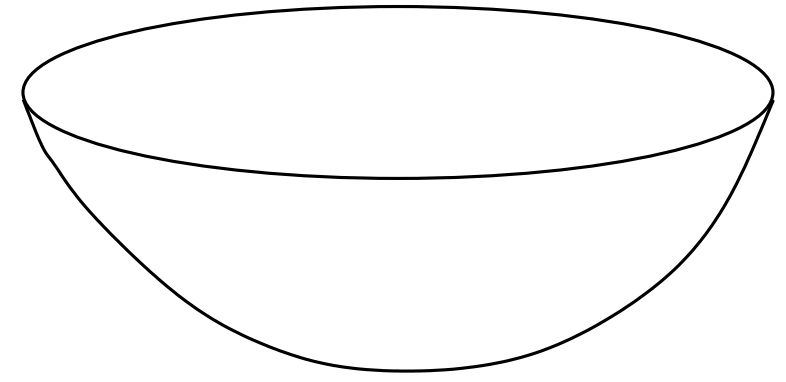

Fig. 1.6 Ilustração geométrica do conjunto de pontos de $M$ tais que $f(p)<a<f(q)$.

(iii) Se $f(q)<a<f(r)$, então $M^{a}$ tem a forma de um cilíndro:

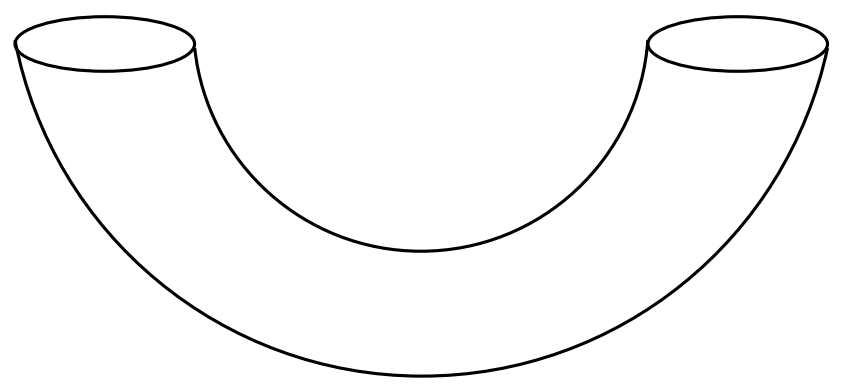

Fig. 1.7 Ilustração geométrica do conjunto de pontos de $M$ tais que $f(q)<a<f(r)$.

(iv) Se $f(r)<a<f(s)$, então $M^{a}$ é essencialmente uma variedade compacta com um "buraco" (genus um) tendo um círculo como fronteira: 


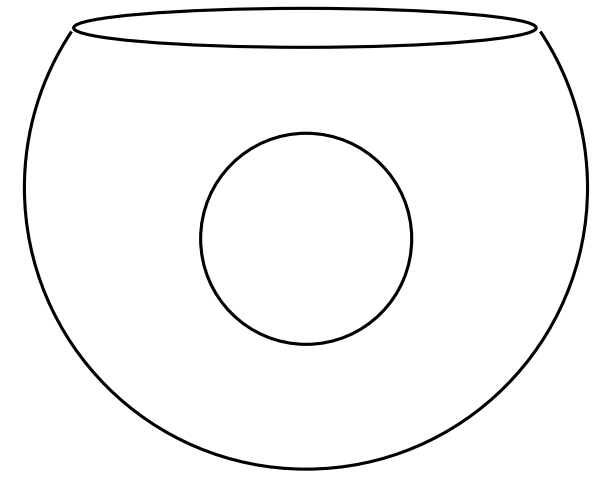

Fig. 1.8 Ilustração geométrica do conjunto de pontos de $M$ tais que $f(r)<a<f(s)$.

(v) Se $f(s)<a$, então $M^{a}$ é o toro inteiro.

As características de $M^{a}$ em cada um dos estágios de $(i)$ a $(v)$ pode ser caracterizada também por sua homotopia e a transição entre os estágios pode ser descrita em termos de anexação de "alças". O estágio (ii) pode ser continuamente deformado e reduzido a um ponto. Passar de (ii) a (iii) é o que se faz ao incluir uma "alça" (detalhes do processo analítico que permite esta inclusão podem ser vistas em [29]):
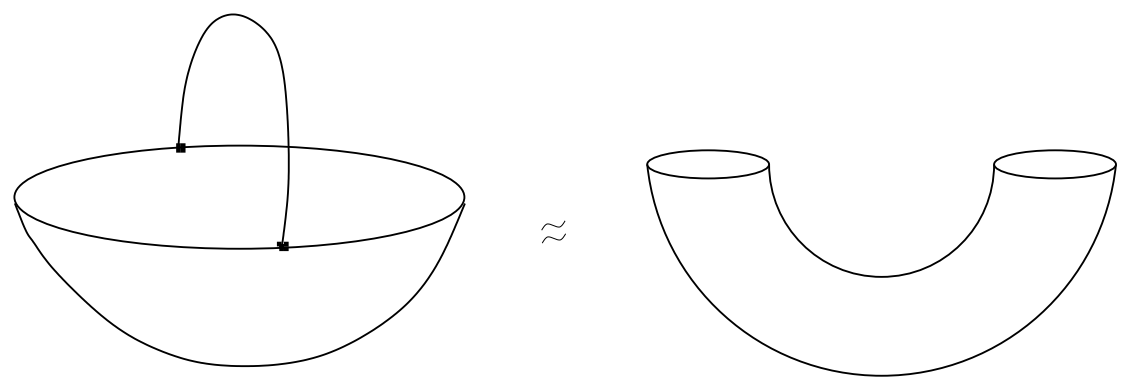

Fig. 1.9 Esquematização do processo de incluir uma "alça" ao conjunto da figura 1.6 para obter-se a figura 1.7.

Para passar de (iii) a (iv) também adiciona-se uma "alça": 

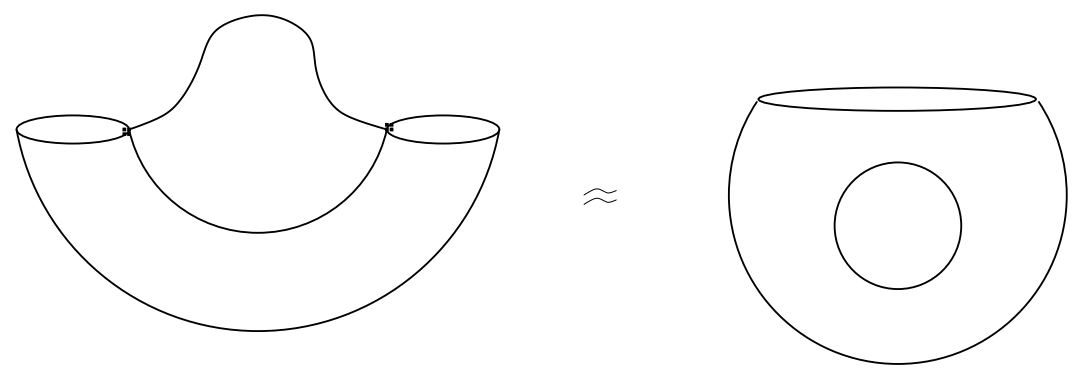

Fig. 1.10 Esquematização do processo de incluir uma "alça" ao conjunto da figura 1.7 para obter-se a figura 1.8 .

O passo final é obtido anexando-se uma "alça bidimensional" à superficie de genus um e bordo sobre o círculo, obtendo-se o toro completo. A dimensão da "alça"a ser anexada depende das características do ponto crítico de $f$. Pelo Lema de Morse [30], nas vizinhanças de um ponto crítico $p$ não degenerado (Hessiano não nulo) de $f$ existe um sistema de coordenadas tal que $f$ pode ser escrita como

$$
f=f(p)-\left(y_{1}\right)^{2}-\ldots-\left(y_{i}\right)^{2}+\left(y_{i+1}\right)^{2}+\ldots+\left(y_{n}\right)^{2}
$$

onde o número $i$ de direções em que a função decresce é chamado indice de f em $p$ e é quem determina a dimensão da "alça"a ser anexada na passagem entre subvariedades $M^{a}$ quando se passa por um ponto crítico de $f$.

Este exemplo simples mostra que há uma conexão estreita entre a característica de pontos críticos não-degenerados de uma função definida sobre uma variedade - especificamente o índice da função em seus pontos críticos - e a topologia da variedade. Esta interrelação entre topologia e o número e característica de pontos críticos de uma função definida sobre a variedade pode ser detalhada em termos das desigualdades de Morse. Estas desigualdades são estabelecidas dadas $M$, uma variedade compacta, e $f$, uma função diferenciável definida em $M$ com pontos críticos isolados e não-degenerados. As desigualdades são [29]:

$$
N_{i} \leq B_{i}, \quad i \geq 0
$$

e

$$
\sum_{i=0}^{\infty}(-1)^{i} N_{i}=\sum_{i=0}^{\infty}(-1)^{i} B_{i},
$$

sendo $B_{i}$ o $i$-ésimo número de Betti da variedade e $N_{i}$ o número de pontos críticos com índice $i$ de $f$. Podemos interpretar (1.24) como o requerimento 
topológico para a existência de pontos críticos enquanto (1.25) funciona como uma limitação ao excesso de pontos críticos [31]. A soma alternada dos números de Betti que aparece no lado direito de (1.25) é a característica de Euler da variedade [32].

Para o caso do toro $T^{2}$, por exemplo, os números de Betti não nulos são $B_{0}=B_{2}=1, B_{1}=2$ [32]. Sabemos, portanto, de (1.24) que há no mínimo um ponto crítico com índice 0 , um com índice 2 e dois pontos críticos com índice 1 para qualquer função diferenciável definida em $T^{2}$. No caso específico da função altura que introduzimos e fazendo referência à figura 1.4.1, o ponto $p$ é o que tem índice nulo, $q$ e $r$ têm índice um e $s$ tem índice dois.

\subsubsection{Teoria de Morse e lentes gravitacionais}

No estudo de lentes gravitacionais estamos interessados em um problema bastante mais complexo do que o que acabamos de ilustrar: estamos estudando pontos críticos do funcional energia definido sobre um espaço de curvas nulas definidas em uma variedade Lorentziana conectando um ponto $p$ a uma curva $\eta$.

Um resultado que sabemos ser válido é [9]:

Teorema 1.21. (Teorema do Índice de Morse) $O$ índice da forma bilinear definida por (1.15) é finito e igual ao número de pontos de $\gamma(\lambda), 0<$ $\lambda<a$, conjugados a $\gamma(0)$, cada um contado com sua multiplicidade.

Como anteriormente notado, a forma bilinear (1.15) desempenha o papel de hessiano do funcional energia em pontos críticos e seu índice (dimensão do subespaço negativo-definido) tem o mesmo papel que o índice da função $f$ do exemplo anterior. Também paralelamente ao caso anterior e à luz do teorema do índice de Morse, $N_{i}$ é o número de geodésicas nulas apontando para o passado conectando o ponto $p$ à curva tipo-tempo $\eta$ que passam por $i$ pontos conjugados antes de alcançar $\eta$, com cada ponto conjugado contado com sua multiplicidade (lembremos que, pelo princípio de Fermat, para cada ponto extremo do funcional energia está associado uma geodésica nula ligando $\eta$ a $p$ ).

O principal obstáculo para estabelecer as desigualdades de Morse também para os espaços de curvas definidas sobre variedades Lorentzianas que nos interessa é contornar as dificuldades encontradas em flexibilizar a condição de compacidade da variedade $M$ e tratar, com efeito, variedades de dimensão infinita sobre as quais um funcional é definido. Os esforços seminais neste sentido 


\section{Lentes Gravitacionais: maquinaria básica}

foram dados pelos trabalhos [20] e [18] que tratam respectivamente da teoria de Morse em variedades de Hilbert e teoria de Morse em variedades Lorentzianas globalmente hiperbólicas. O resumo desta longa história é que, para que seja possível estabelecer relações entre os números $N_{i}$ e números determinados pela topologia do espaço de curvas $\Omega_{p, \eta}$, o funcional energia além de ter apenas pontos críticos não degenerados tem que satisfazer condições de limitação inferior e a chamada de condição de Palais-Smale, que, embora bastante técnica, tem a função de fazer uma mímica das boas propriedades de variedades compactas no caso de variedades de dimensão infinita [33]. Demonstrou-se em [19] que o funcional energia que utilizamos satisfaz todas as condições necessárias para ser uma função de Morse em variedades Lorentzianas globalmente hiperbólicas e portanto valem neste caso as desigualdades de Morse (1.24) e (1.25). (É necessária uma visão mais sofisticada de $\Omega_{p, \eta}$ que a aqui apresentada para que se possam definir os números de Betti, mas isso não será nosso assunto).

As conseqüências da validade dessas desigualdades são enormes para a teoria das lentes gravitacionais. Por um lado, essas desigualdades são elementos centrais na demonstração de que configurações de lentes em espaços globalmente hiperbólicos (ou seja, defletores necessariamente transparentes) geram sempre um número ímpar de imagens, contanto que não existam horizontes de partículas ou que a topologia espacial seja trivial [7]. Por outro lado, a teoria de Morse de maneira geral ilumina a teoria de lentes gravitacionais sob um ângulo muito curioso, mostrando que a resposta teórica a uma pergunta do tipo "quantas imagens de uma fonte luminosa podem ser vistas por um observador" depende não apenas das características da métrica e estrutura causal do espaço-tempo, mas está codificada na topologia de um espaço de curvas variacionais. Muito embora não tenhamos outro meio de conhecer essa topologia que através do número de imagens observadas de uma fonte (no caso ideal em que todas as imagens possíveis são observadas), o fato de poder ser modelado nestes termos faz do problema de lentes gravitacionais um exemplo de elegância na física teórica moderna.

A eventual simplicidade relativa associada a essa matematização do problema de lentes vem do fato que estamos interessados na homologia simplicial do espaço das curvas, ou seja, estamos interessados em descrever a topologia deste espaço em termos de somas de poliedros [34,35]. Um tipo semelhante de estrutura de homologia aparece também no estudo de formas sobre varie- 
dades, dando origem (dualmente) à cohomologia de de Rham, em termos da qual invariantes topológicos também podem ser determinados [36]. O poder destas estruturas e dos invariantes topológicos revela-se especialmente útil na física quando associados à teoria de Hodge e o estudo de formas harmônicas. Um exemplo disso pode ser visto na notável referência [37], onde apresentase uma derivação das desigualdades de Morse partindo-se de questões sobre a existência de estados de vácuo associados a hamiltonianos supersimétricos.

Por ser o instrumento teórico mais adequado ao estudo de sistemas que apresentam pontos críticos, que são os elementos finais que nos conduziram à teoria de Morse no estudo de lentes gravitacionais, a mecânica estatística também se beneficia dos mesmos métodos aqui utilizados e que permitem, naquele contexto, formular por exemplo a hipótese de que transições de fase estão associadas a mudanças na topologia do espaço de configurações [38]. Também como aplicação da teoria de Morse no estudo de transições de fase - e talvez a mais antiga aplicação da teoria de Morse na física - está a determinação de quais possíveis subgrupos de um grupo de simetrias de um cristal são acessíveis após a quebra da simetria inicial [39] e singularidades de van Hove [40]. 


\section{2}

\section{Evolução de feixes de geodésicas nulas e lentes gravitacionais}

Estudando métodos variacionais aplicados a geodésicas nulas deparamo-nos de maneira incontornável com a equação de Jacobi quando tentamos caracterizar os pontos estacionários do funcional energia. Graças à interpretação geométrica que têm, além de determinar pontos conjugados ao longo de uma geodésica, campos de Jacobi mostram-se indispensáveis como instrumentos de descrição da deformação que feixes de geodésicas sofrem em sua propagação em geometrias não triviais, o que corresponde a uma das tarefas daqueles que estudam lentes gravitacionais, em especial as lentes fracas.

O estudo destes campos de Jacobi envolve, no entanto, uma diversidade de sutilezas em parte advindas de vetores nulos serem peculiares na geometria Lorentziana, mas também oriundas do fato notado na Seção A.7 do Apêndice A, especificamente, de que dada uma geodésica $\gamma(\lambda)$ em um espaço-tempo, $\gamma^{\prime}(\lambda)$ e $\lambda \gamma^{\prime}(\lambda)$ são campos de Jacobi não triviais ao longo de $\gamma$ mas que não indicam separação em direções tipo-espaço entre geodésicas em um feixe. Finalmente, há a restrição observacional de que uma imagem vista no céu é um objeto bidimensional e portanto tem-se que criar uma maneira de extrair as duas dimensões associadas a essas imagens das quatro dimensões inerentes aos objetos surgidos na descrição de fenômenos físicos em Relatividade Geral. Veremos que esses três fatores estão intimamente relacionados e também que os dois primeiros conduzem à construção de um espaço vetorial bidimensional que justamente resolve o terceiro problema listado. A construção deste espaço bidimensional, como descrita na seção 4.2 de [15], é um pouco técnica mas traz consigo a possibilidade de tratar o problema das lentes de maneira precisa, que é, afinal, nosso objetivo.

Mostraremos como construir a partir das restrições dadas um espaço bidimensional (também chamado de "espaço dos screen vectors"), como restringir 
a métrica, derivada covariante e curvatura a este espaço, assim como as particularidades da equação de Jacobi a ele restrita. Utilizaremos o conceito de tensores de Jacobi para derivar as equações que regem a deformação de um feixe de geodésicas quando se propaga em um espaço-tempo. Dados os instrumentos que teremos desenvolvido, seremos capazes de discutir a relação existente entre as condições de energia e a existência de pontos conjugados ao longo de geodésicas e, consequentemente, com a existência de imagens múltiplas.

Os desenvolvimentos apresentados na seqüência deste capítulo pretendem clarificar a derivação apresentada por [1] das equações básicas para a descrição de lentes gravitacionais em espaços-tempo arbitrários. Entendemos que a derivação por eles apresentada, embora leve às equações que de fato descrevem o sistema físico de interesse, seguem manipulações que não são válidas no contexto em que são empregadas.

O presente capítulo deu origem à publicação [5].

\subsection{O problema da derivação de [1]: interpretações da equação de Jacobi}

A equação de Jacobi surge, como vimos, de diversas maneiras em geometria e relatividade geral e, em especial no estudo de lentes gravitacionais, desempenha papel protagonista. Este protagonismo justifica-se não apenas por sua interpretação de maneira como geodésicas vizinhas afastam-se (ou aproximam-se) umas das outras em sua propagação sob efeito da curvatura mas também por sua notória contribuição para a interpretação da forma do índice e consequente caracterização dos extremos do funcional energia. Deve-se notar, no entanto, que a equação de Jacobi, que merece ser reescrita,

$$
\frac{D^{2} J}{d \lambda^{2}}+R\left(\frac{d \gamma}{d \lambda}, J(\lambda)\right) \frac{d \gamma}{d \lambda}=0
$$

tem soluções que são apenas finalmente fixadas, se existirem, quando impostas condições iniciais.

As interpretações das soluções da equação de Jacobi dependem portanto das condições iniciais: sabe-se, como mostrado na Seção A.7 do Apêndice A, que $\gamma^{\prime}(\lambda)$ e $\lambda \gamma^{\prime}(\lambda)$ sempre são campos de Jacobi ao longo de $\gamma$ e certamente não podem ser interpretadas como descrevendo como duas geodésicas próximas afastam-se uma da outra. Sucede que uma outra interpretação para soluções das equações de Jacobi pode ser construída notando-se que, sob certas condições, a equação de Jacobi admite uma "integral primeira". Consideremos, para tanto, 
36 Evolução de feixes de geodésicas nulas e lentes gravitacionais

um campo de vetores $T \in \mathfrak{X}(M)$ obtido como tangente a uma família uniparamétrica de curvas em $M$ e seja $X$ um campo em $\mathfrak{X}(M)$ que satisfaça

$$
£_{T} X=0 .
$$

Especificamente estamos pensando no exemplo apresentado por [41] em que $T$ é o campo de velocidades de uma congruência de geodésicas e $X$ representa um vetor tipo-espaço ortogonal ao campo de velocidades que indica o desvio entre um dos elementos da congruência e um raio central $\gamma_{0}$ previamente fixado. Derivando-se $[T, X]=0$ em relação ao parâmetro afim do raio central, obtemos:

$$
\frac{D^{2} X}{d t^{2}}+R(T, X) T=0
$$

que é a equação de Jacobi. Neste caso, vale notar, uma condição inicial do tipo $X(0)=0$ não pode ser aceita pois curvas em uma congruência não podem interceptar-se, sob a pena de serem idênticas.

A derivação das equações centrais no estudo de lentes gravitacionais apresentada em [1], entretanto, advém exatamente desses passos descritos: diferenciase uma relação equivalente a $[T, X]=0$ para obter uma equação de Jacobi e em seguida aplica-se a ela condições iniciais inconsistentes com a equação de que se partiu. Na seqüência do presente capítulo apresentaremos uma derivação alternativa das mesmas equações centrais sem incorrer no mesmo tipo de falha.

\subsection{O espaço de vetores adequado para o estudo de nosso problema}

Seja $\gamma:[0, a] \rightarrow M$ uma geodésica nula tal que $\gamma(0)=p$. Denotaremos $\gamma^{\prime}(0)=$ $k$. Podemos introduzir uma base $\left\{E_{0}, E_{1}, E_{2}, E_{3}\right\}$ (não ortonormal) em $T_{p} M$ como na seção 4.2 de [15]: tomamos $E_{0}=k$ e $E_{3}=l$, com $l$ um outro vetor nulo satisfazendo $\langle k, l\rangle=-1$ e $E_{1}$ e $E_{2}$ são dois vetores tipo-espaço unitários e ortogonais. Devemos notar que devido à particularidade dessa base, um vetor arbitrário em $T_{p} M$ poderá ser decomposto, de maneira não única, como a soma de uma componente ortogonal a $k$ e uma componente não-ortogonal a ele. Apesar de não ortonormal, $\left\{E_{0}, E_{1}, E_{2}, E_{3}\right\}$ constitui uma base e, por conseguinte, seus vetores são linearmente independentes. Segue então que para $\lambda \in\left(0, \lambda_{0}\right)$, sendo $\lambda_{0}$ o parâmetro correspondente ao primeiro ponto conjugado a $\gamma(0)$ ao longo de $\gamma$, o conjunto de campos de Jacobi constituído tomando cada um dos vetores acima referidos como $J_{i}^{\prime}(0)$ evolui independentemente. 
Notemos que se $J^{\prime}(0) \in \operatorname{span}(l)$, então $\left\langle J^{\prime}(0), \gamma^{\prime}(0)\right\rangle \neq 0$ e, pelo Corolário A.40, $\left\langle J(\lambda), \gamma^{\prime}(\lambda)\right\rangle \neq 0, \lambda \in\left(0, \lambda_{0}\right)$. Pela continuidade da forma bilinear podemos separar no conjunto dos campos de Jacobi em $T_{\gamma(\lambda)} M$ o campo que satisfaz $\left\langle J(\lambda), \gamma^{\prime}(\lambda)\right\rangle \neq 0$ dos que satisfazem $\left\langle J(\lambda), \gamma^{\prime}(\lambda)\right\rangle=0$ (por conseqüência da Proposição A.10). De fato, podemos considerar o conjunto dos vetores que são imagem inversa de 0 pela aplicação $\left\langle., \gamma^{\prime}(\lambda)\right\rangle$ (que, com efeito, é um espaço vetorial) que denotaremos por $\tilde{T}_{\gamma(\lambda)} M$.

Como pretendemos descrever o modo como geodésicas que se iniciam em um dado ponto afastam-se umas das outras, devemos centrar nossa análise em $\tilde{T}_{\gamma(\lambda)} M$. Mais além, os objetos dignos de nossa atenção são as classes de equivalência de vetores em $\tilde{T}_{\gamma(\lambda)} M$, nas quais dois vetores são considerados equivalentes se sua diferença é proporcional a $\gamma^{\prime}(\lambda)$. Vejamos mais precisamente no que consistem essas classes:

Sejam $T_{\gamma(\lambda)} M$ o espaço tangente ao ponto $\gamma(\lambda)$ e os vetores em $T_{\gamma(\lambda)} M$ ortogonais a $\gamma^{\prime}(\lambda)$ formando um espaço tridimensional que denotaremos por $\tilde{T}_{\gamma(\lambda)} M$, como antes. Podemos definir em $\tilde{T}_{\gamma(\lambda)} M$ a seguinte relação de equivalência: dados $V, W \in \tilde{T}_{\gamma(\lambda)} M, V \sim W$ se $V-W \in \operatorname{span}\left(\gamma^{\prime}(\lambda)\right)$. É usual denotar por $\tilde{T}_{\gamma(\lambda)} M / \operatorname{span}\left(\gamma^{\prime}(\lambda)\right)$ o conjunto das classes de equivalência de $\tilde{T}_{\gamma(\lambda)} M$ pela relação $\sim$. Denotaremos por $[V] \in \tilde{T}_{\gamma(\lambda)} M / \operatorname{span}\left(\gamma^{\prime}(\lambda)\right)$ a classe de equivalência que contém o vetor $V \in \tilde{T}_{\gamma(\lambda)} M$. É sabidamente possível transformar $\tilde{T}_{\gamma(\lambda)} M / \operatorname{span}\left(\gamma^{\prime}(\lambda)\right)$ em um espaço vetorial sobre $\mathbb{R}$ (ou $\mathbb{C}$ ), bastando para isso definir em $\tilde{T}_{\gamma(\lambda)} M / \operatorname{span}\left(\gamma^{\prime}(\lambda)\right)$ uma soma e um produto por escalares [42]. O vetor nulo é a classe de equivalência [0] que contém o vetor 0 e todos os vetores de $\tilde{T}_{\gamma(\lambda)} M$ proporcionais a $\gamma^{\prime}(\lambda)$, ou seja, como subconjunto de $\tilde{T}_{\gamma(\lambda)} M$, a classe [0] é o $\operatorname{span}\left(\gamma^{\prime}(\lambda)\right)$. Além disso é possível mostrar que, dados $V, W \in \tilde{T}_{\gamma(\lambda)} M$, $\alpha \in \mathbb{R}(\mathbb{C})$, as operações $[V]+[W]=[V+W]$ e $\alpha[V]=[\alpha V]$ são bem definidas. O espaço $\tilde{T}_{\gamma(\lambda)} M / \operatorname{span}\left(\gamma^{\prime}(\lambda)\right)$ é chamado de quociente de $\tilde{T}_{p} M$ por $\operatorname{span}\left(\gamma^{\prime}(\lambda)\right) . \tilde{T}_{\gamma(\lambda)} M / \operatorname{span}\left(\gamma^{\prime}(\lambda)\right)$ é bidimensional e nele estão contidos os vetores pelos quais nos interessamos. Os elementos deste espaço são chamados por vezes na literatura de screen vectors ou Sachs beine [14].

\subsection{Restrição da métrica, derivada e curvatura}

Dadas as restrições geométricas impostas, obtivemos $\tilde{T}_{\gamma(\lambda)} M / \operatorname{span}\left(\gamma^{\prime}(\lambda)\right)$ como o espaço no qual devemos tratar o problema em que nos interessamos. Restanos mostrar como a métrica, a derivada covariante e a curvatura neste espaço relacionam-se com os objetos correspondentes em $T_{\gamma(\lambda)} M$. 
38 Evolução de feixes de geodésicas nulas e lentes gravitacionais

Métrica. Sejam $U, V \in \tilde{T}_{\gamma(\lambda)} M$. Se denotamos a métrica restrita ao quociente $\tilde{T}_{\gamma(\lambda)} M / \operatorname{span}\left(\gamma^{\prime}(\lambda)\right)$ como $\langle., \cdot\rangle_{q}$,

$$
\langle[U],[V]\rangle_{q}=\langle U, V\rangle
$$

que está bem definida pois dados $U=\tilde{U}+\alpha \gamma^{\prime}(\lambda), V=\tilde{V}+\beta \gamma^{\prime}(\lambda)$,

$$
\langle U, V\rangle=\left\langle\tilde{U}+\alpha \gamma^{\prime}, \tilde{V}+\beta \gamma^{\prime}\right\rangle=\langle\tilde{U}, \tilde{V}\rangle
$$

que obviamente não depende do elemento escolhido dentro da classe.

Vale notar que $\langle., .\rangle_{q}$ é positiva-definida em $\tilde{T}_{\gamma(\lambda)} M / \operatorname{span}\left(\gamma^{\prime}(\lambda)\right)$.

Derivada covariante. Seja $\mathfrak{X}_{q}(\gamma)$ o conjunto de campos de vetores suaves por partes em $\tilde{T}_{\gamma(\lambda)} M / \operatorname{span}\left(\gamma^{\prime}(\lambda)\right), \gamma:[0, a] \rightarrow M$. Dado $Y \in \mathfrak{X}_{q}(\gamma)$,

$$
\nabla_{k} Y=\left[\nabla_{k} W\right]
$$

para algum $W \in \tilde{T}_{\gamma(\lambda)} M$ tal que $[W]=Y$.

Para ver que a derivada não depende do objeto particular escolhido na classe, tomemos $\tilde{W} \in \tilde{T}_{\gamma(\lambda)} M$ satisfazendo $[\tilde{W}]=Y$ e uma função suave $f$ : $[0, a] \rightarrow \mathbb{R}$. Então,

$$
\nabla_{\gamma^{\prime}} \tilde{W}=\nabla_{\gamma^{\prime}} W+\nabla_{\gamma^{\prime}}\left(f \gamma^{\prime}\right)=\nabla_{\gamma^{\prime}} W+\frac{d f}{d \lambda} \gamma^{\prime}
$$

dado que $\gamma$ é suposta uma geodésica. Portanto $\left[\nabla_{\gamma^{\prime}} W\right]=\left[\nabla_{\gamma^{\prime}} \tilde{W}\right]$ para todo $\lambda \in[0, a]$. Além disso a derivada é compatível com a métrica.

Curvatura. Dados $X \in \tilde{T}_{\gamma(\lambda)} M / \operatorname{span}\left(\gamma^{\prime}(\lambda)\right), V \in \tilde{T}_{\gamma(\lambda)} M$ tal que $[V]=X$,

$$
\bar{R}\left(X, \gamma^{\prime}\right) \gamma^{\prime}=\left[R\left(V, \gamma^{\prime}\right) \gamma^{\prime}\right]
$$

Como anteriormente, tomemos $\tilde{V} \in \tilde{T}_{\gamma(\lambda)} M,[\tilde{V}]=X$,

$$
R\left([\tilde{V}], \gamma^{\prime}\right) \gamma^{\prime}=R\left(X+\alpha \gamma^{\prime}, \gamma^{\prime}\right) \gamma^{\prime}=R\left(X, \gamma^{\prime}\right) \gamma^{\prime}+\alpha R\left(\gamma^{\prime}, \gamma^{\prime}\right) \gamma^{\prime}=R\left(X, \gamma^{\prime}\right) \gamma^{\prime} .
$$

Ainda, dado $U \in \tilde{T}_{\gamma(\lambda)} M,[U]=Y$,

$$
\langle\bar{R}(X, k) k, Y\rangle_{q}:=\langle[R([V], k) k],[U]\rangle_{q}=\langle R(V, k) k, U\rangle .
$$




\subsection{Classes de Jacobi}

Descreveremos os equivalentes a campos de Jacobi para o espaço quociente $\tilde{T}_{\gamma(\lambda)} M / \operatorname{span}\left(\gamma^{\prime}(k)\right)$. Os desenvolvimentos dessa Seção são baseados em [43].

Definição 2.1 Um campo suave $X \in \mathfrak{X}_{q}$ é dito uma classe de Jacobi em $\tilde{T}_{\gamma(\lambda)} M / \operatorname{span}\left(\gamma^{\prime}(k)\right)$ se satisfaz a equação diferencial

$$
X^{\prime \prime}+\bar{R}\left(X, \gamma^{\prime}\right) \gamma^{\prime}=\left[\gamma^{\prime}\right]
$$

com a derivada e curvatura dadas respectivamente por (2.5) e (2.6).

Interpretando esta definição, devemos dizer que as classes de Jacobi descreverão o afastamento entre geodésicas em direções tipo-espaço ortogonais a $\gamma^{\prime}$. Qualquer campo vetorial que satisfaça o lado esquerdo da equação (2.8) sendo proporcional a $\gamma^{\prime}$, ou seja, uma pré-geodésica com velocidade múltipla de $\gamma^{\prime}$, será colocado na classe de equivalência do campo $\gamma^{\prime}$.

Os lemas que seguem estabelecem a relação entre campos de Jacobi em $\tilde{T}_{\gamma(\lambda)} M$ e $\tilde{T}_{\gamma(\lambda)} M / \operatorname{span}\left(\gamma^{\prime}(k)\right)$.

Lema 2.2 Seja $X$ uma classe de Jacobi em $\tilde{T}_{\gamma(\lambda)} M / \operatorname{span}\left(\gamma^{\prime}(k)\right)$. Então existe em campo de Jacobi suave $V \in \tilde{T}_{\gamma(\lambda)} M$ com $[V]=X$. Também, se $V$ é um campo de Jacobi em $\tilde{T}_{\gamma(\lambda)} M$, então $[V]$ é uma classe de Jacobi em $\tilde{T}_{\gamma(\lambda)} M / \operatorname{span}\left(\gamma^{\prime}(k)\right)$.

\section{Demonstração}

A segunda parte do lema segue de

$$
V^{\prime \prime}+R\left(V, \gamma^{\prime}\right) \gamma^{\prime}=0 \Rightarrow\left[V^{\prime \prime}+R\left(V, \gamma^{\prime}\right) \gamma^{\prime}\right]=[V]^{\prime \prime}+\bar{R}\left([V], \gamma^{\prime}\right) \gamma^{\prime}=0 .
$$

Para a demonstração da primeira afirmação consideremos $X$ uma classe de Jacobi e seja $W$ um campo suave de vetores em $\tilde{T}_{\gamma(\lambda)} M$ tal que $[W]=X$. Como $X^{\prime \prime}+\bar{R}\left(X, \gamma^{\prime}\right) \gamma^{\prime}=\left[\gamma^{\prime}\right]$ em $\mathfrak{X}_{q}$, existe uma função suave $f:[0, a] \rightarrow \mathbb{R}$ tal que $W^{\prime \prime}+R\left(W, \gamma^{\prime}\right) \gamma^{\prime}=f \gamma^{\prime}$. Seja agora $h:[0, a] \rightarrow \mathbb{R}$ uma segunda função suave tal que $h^{\prime \prime}=f$ e tomemos $V=W-h \gamma^{\prime}$. Então $[V]=X$ e

$$
\begin{aligned}
f \gamma^{\prime} & =W^{\prime \prime}+R\left(W, \gamma^{\prime}\right) \gamma^{\prime}=\left(V+h \gamma^{\prime}\right)^{\prime \prime}+R\left(\left(V+h \gamma^{\prime}\right), \gamma^{\prime}\right) \gamma^{\prime}=V^{\prime \prime}+h^{\prime \prime} \gamma^{\prime}+R\left(V, \gamma^{\prime}\right) \gamma^{\prime} \\
& =V^{\prime \prime}+f \gamma^{\prime}+R\left(V, \gamma^{\prime}\right) \gamma^{\prime}
\end{aligned}
$$

e, portanto, $V^{\prime \prime}+R\left(V, \gamma^{\prime}\right) \gamma^{\prime}=0$ 
40 Evolução de feixes de geodésicas nulas e lentes gravitacionais

Lema 2.3 Seja $X \in \mathfrak{X}_{q}(k)$ uma classe de Jacobi com $X(0)=\left[\gamma^{\prime}(0)\right]$ e $X(a)=$ $\left[\gamma^{\prime}(a)\right]$. Então existe um único campo de Jacobi $V \in \tilde{T}_{\gamma(\lambda)} M$ satisfazendo $[V]=$ $X$ e $V(0)=V(a)=0$.

\section{Demonstração}

Do Lema (2.2) sabemos que existe um campo de Jacobi $W \in \tilde{T}_{\gamma(\lambda)} M$ com $[W]=X$. Não sabemos, entretanto, que $W(0)=W(a)=0$. Entretanto, dadas $\alpha, \beta$ duas constantes reais, então $W+\alpha \gamma^{\prime}+\beta \lambda \gamma^{\prime}$ é também um campo de Jacobi em $\tilde{T}_{\gamma(\lambda)} M \operatorname{com}\left[W+\alpha \gamma^{\prime}+\beta \lambda \gamma^{\prime}\right]=X$. Como $[W]=X$ e $X(0)=\left[\gamma^{\prime}(0)\right]$, $X(a)=\left[\gamma^{\prime}(a)\right]$, sabemos que $W(0)=c_{1} \gamma^{\prime}(0)$ e $W(a)=c_{2} \gamma^{\prime}(a)$, para $c_{1}, c_{2} \in \mathbb{R}$. Escolhendo

$$
\alpha=-c_{1} \quad \beta=\frac{1}{a}\left(c_{1}-c_{2}\right)
$$

segue facilmente que $V=W+\alpha \gamma^{\prime}+\beta \lambda \gamma^{\prime}$ satisfaz $V(0)=V(a)=0$.

Para constatarmos a unicidade, suponha que $\tilde{V}$ seja um segundo campo de Jacobi em $\tilde{T}_{\gamma(\lambda)} M \operatorname{com}[\tilde{V}]=X$ e $\tilde{V}(0)=\tilde{V}(a)=0$. Então $W=\tilde{V}-V$ é um campo de Jacobi da forma $W=h k$ com $W(0)=W(a)=0$. Como $0=W^{\prime \prime}+R\left(W, \gamma^{\prime}\right) \gamma^{\prime}=h^{\prime \prime} \gamma^{\prime}+h R\left(\gamma^{\prime}, \gamma^{\prime}\right) \gamma^{\prime}=h^{\prime \prime} \gamma^{\prime}$, segue que $h$ é uma função afim. Como $h(0)=h(a)$, devemos ter $h=0$ identicamente e $\tilde{V}=V$ como pretendíamos.

Estamos agora em condições de definir campos conjugados de classes de Jacobi no espaço quociente. Chamamos atenção, antes, ao fato de que se um campo de Jacobi $J$ ao longo de $\gamma$ satisfaz $J\left(\lambda_{1}\right)=J\left(\lambda_{2}\right)=0$ para $\lambda_{1} \neq \lambda_{2}$, então $J \in \tilde{T}_{\gamma(\lambda)} M$.

Definição 2.4 Seja $\gamma:[0, a] \rightarrow(M, g)$ uma geodésica nula. Para $\lambda_{1} \neq \lambda_{2}$ em $[0, a], \lambda_{1}$ e $\lambda_{2}$ são ditos conjugados ao longo de $\gamma$ se existir uma classe de Jacobi $X \neq\left[\gamma^{\prime}\right]$ em $\mathfrak{X}(\gamma)$ com $W\left(\lambda_{1}\right)=\left[\gamma^{\prime}\left(\lambda_{1}\right)\right]$ e $W\left(\lambda_{2}\right)=\left[\gamma^{\prime}\left(\lambda_{2}\right)\right]$. Também $\lambda_{1} \in(0, a]$ é dito um ponto conjugado de $\gamma$ se $\lambda=0$ e $\lambda_{1}$ são conjugados ao longo de $\gamma$.

Devemos notar que, em virtude do Lema 2.3, a pontos conjugados na classe de equivalência são também pontos conjugados em $\tilde{T}_{\gamma(\lambda)} M$.

\subsection{Tensores de Jacobi}

Definiremos nesta seção, também baseados em [43], o conceito de tensor de Jacobi. A importância destes objetos no estudo de propriedades ópticas é crucial uma vez que é em termos deles que se pode definir a expansão, vorticidade e 
cisalhamento de feixes de geodésica. Tensores de Jacobi também são centrais no estudo de singularidades associadas a geodésicas nulas pois é através da manipulação desse tipo de objeto que se pode derivar a equação de Raychaudhuri para feixes de geodésicas nulas. No que segue imediatamente apenas definiremos os tensores de Jacobi e mostraremos como construir um tal tensor a partir de uma classe de Jacobi.

Um campo de tensores $(1,1) A(\lambda)$ em $\tilde{T}_{\gamma(\lambda)} M$ é uma transformação linear

$$
A=A(\lambda): \tilde{T}_{\gamma(\lambda)} M \rightarrow \tilde{T}_{\gamma(\lambda)} M
$$

para cada $\lambda \in[0, a]$. Com isso, se $V \in \tilde{T}_{\gamma(\lambda)} M, A(\lambda)(V) \in \tilde{T}_{\gamma(\lambda)} M$.

Um endomorfismo composto $R A(\lambda): \tilde{T}_{\gamma(\lambda)} M \rightarrow \tilde{T}_{\gamma(\lambda)} M$ pode ser definido fazendo

$$
R A(\lambda)(V)=R\left(A(\lambda)(V), \gamma^{\prime}(\lambda)\right) \gamma^{\prime}(\lambda) \in \tilde{T}_{\gamma(\lambda)} M, \quad \forall V \in \tilde{T}_{\gamma(\lambda)} M .
$$

O campo de tensores $A(\lambda)$ pode ser definido de maneira suave se os mapas

$$
[0, a] \ni \lambda \mapsto A\left(E_{j}\right)(\lambda) \in \tilde{T}_{\gamma(\lambda)} M, j=1,2
$$

são suaves.

Escrevendo

$$
A\left(E_{j}\right)=\sum_{i=1}^{2} f_{j}^{i} E_{i}+f_{j} \gamma^{\prime}
$$

onde $f_{j}^{i}, f_{j}:[0, a] \rightarrow \mathbb{R}$ são suaves, podemos definir o campo tensorial $(1,1)$ $A^{\prime}(\lambda): \tilde{T}_{\gamma(\lambda)} M \rightarrow \tilde{T}_{\gamma(\lambda)} M$ por

$$
A^{\prime}\left(E_{j}\right)=\sum_{i=1}^{2}\left(f_{j}^{i}\right)^{\prime} E_{i}+f_{i}^{\prime} \gamma^{\prime} \quad i, j=1,2 .
$$

Com essas regras, a composição de tensores $(1,1)$ pode ser identificada com a multiplicação de matrizes das funções componentes $f_{j}^{i}$ e $f_{j}$ :

$$
(A B)^{\prime}=A^{\prime} B+A B^{\prime}
$$

$A, B \in \tilde{T}_{\gamma(\lambda)} M$.

No caso em que $A$ é não singular, $A A^{-1}=\mathbb{1}$, tem-se

$$
\left(A^{-1}\right)^{\prime}=-A^{-1} A^{\prime} A^{-1} .
$$


42 Evolução de feixes de geodésicas nulas e lentes gravitacionais

A restrição de campos tensoriais a $\tilde{T}_{\gamma(\lambda)} M / \operatorname{span}\left(\gamma^{\prime}(\lambda)\right)$ pode ser feita definindo $\bar{A}(\lambda): \tilde{T}_{\gamma(\lambda)} M / \operatorname{span}\left(\gamma^{\prime}(\lambda)\right) \rightarrow \tilde{T}_{\gamma(\lambda)} M / \operatorname{span}\left(\gamma^{\prime}(\lambda)\right)$ como uma transformação linear para cada $\lambda \in[0, a]$ que transforma classes de vetores em classes de vetores. Como anteriormente,

$$
(\overline{A B})^{\prime}=\bar{A}^{\prime} \bar{B}+\bar{A} \bar{B}^{\prime}
$$

e, se $\bar{A}$ é não singular,

$$
\left(\bar{A}^{-1}\right)^{\prime}=-(\bar{A})^{-1} \bar{A}^{\prime}(\bar{A})^{-1} .
$$

Como $\langle., .\rangle_{q}: \tilde{T}_{\gamma(\lambda)} M / \operatorname{span}\left(\gamma^{\prime}(\lambda)\right) \times \tilde{T}_{\gamma(\lambda)} M / \operatorname{span}\left(\gamma^{\prime}(\lambda)\right) \rightarrow \mathbb{R}$ é positivadefinida e simétrica, podemos definir o adjunto $\bar{A}^{*}(\lambda)$ de um campo de tensores $(1,1) \bar{A}$ dado:

$$
\langle\bar{A}(W), Z\rangle_{q}=\left\langle\bar{A}^{*}(Z), W\right\rangle_{q} \quad \forall W, Z \in \tilde{T}_{\gamma(\lambda)} M / \operatorname{span}\left(\gamma^{\prime}(\lambda)\right), \forall \lambda \in[0, a] .
$$

Podemos também restringir o endomorfismo $R A$ às classes: $\bar{R} \bar{A}: \tilde{T}_{\gamma(\lambda)} M / \operatorname{span}\left(\gamma^{\prime}(\lambda)\right) \rightarrow$ $\tilde{T}_{\gamma(\lambda)} M / \operatorname{span}\left(\gamma^{\prime}(\lambda)\right)$ :

$$
\bar{R} \bar{A}(W)=\bar{R}\left(\bar{A}(W), \gamma^{\prime}\right) \gamma^{\prime}
$$

O kernel do campo de tensores $(1,1) \bar{A}: \tilde{T}_{\gamma(\lambda)} M / \operatorname{span}\left(\gamma^{\prime}(\lambda)\right) \rightarrow \tilde{T}_{\gamma(\lambda)} M / \operatorname{span}\left(\gamma^{\prime}(\lambda)\right)$ é o espaço vetorial

$$
\operatorname{ker}(\bar{A}(\lambda))=\left\{W \in \tilde{T}_{\gamma(\lambda)} M / \operatorname{span}\left(\gamma^{\prime}(\lambda)\right) \mid \bar{A}(\lambda)(W)=\left[\gamma^{\prime}(\lambda)\right]\right\} .
$$

Um campo $(1,1)$ de tensores $\bar{A}$ é um campo nulo de tensores, denotado $\bar{A}=0$, se $\bar{A}(\lambda)(W)=\left[\gamma^{\prime}(\lambda)\right], \forall W \in \tilde{T}_{\gamma(\lambda)} M / \operatorname{span}\left(\gamma^{\prime}(\lambda)\right)$ e todo $\lambda \in[0, a]$.

Definição 2.5 Um campo de tensores $(1,1) \bar{A}: \tilde{T}_{\gamma(\lambda)} M / \operatorname{span}\left(\gamma^{\prime}(\lambda)\right) \rightarrow \tilde{T}_{\gamma(\lambda)} M / \operatorname{span}\left(\gamma^{\prime}(\lambda)\right)$ é dito ser um tensor de Jacobi se $\bar{A}$ satisfaz as condições

$$
\bar{A}^{\prime \prime}+\bar{R} \bar{A}=0
$$

$e$

$$
\operatorname{ker}(\bar{A}(\lambda)) \cap \operatorname{ker}\left(\bar{A}^{\prime}(\lambda)\right)=\left\{\left[\gamma^{\prime}(\lambda)\right]\right\} \quad \forall \lambda \in[0, a] .
$$


Se $Y \in \mathfrak{X}_{q}(\gamma)$ é uma classe de vetores paralelos ao longo de $\gamma$, então $J=\bar{A}(Y)$ é uma classe de Jacobi ao longo de $\gamma$.

Se $Y_{1}, Y_{2}$ são linearmente independentes, então $\bar{A}\left(Y_{1}\right), \bar{A}\left(Y_{2}\right)$ são linearmente independentes no seguinte sentido: se $\alpha_{1}, \alpha_{2}$ são duas constantes reais tais que

$$
\sum_{i=1}^{2} \alpha_{i} \bar{A}\left(Y_{i}\right)(\lambda)=\left[\gamma^{\prime}(\lambda)\right]
$$

para todo $\lambda \in[0, a]$, então $\alpha_{1}=\alpha_{2}=0$.

\subsubsection{Construção de um tensor de Jacobi a partir de classes de Jacobi}

Sejam $E_{1}, E_{2}$ campos paralelos tipo-espaço em $T_{\gamma(\lambda)} M$. Sejam $\bar{E}_{i}=\left[E_{i}\right]$ as classes paralelas de vetores correspondentes em $\mathfrak{X}_{q}(\gamma)$. Sejam também $\bar{J}_{1}, \bar{J}_{2}$ classes de Jacobi ao longo de $\gamma$ com condições iniciais $\bar{J}_{i}(0)=\left[\gamma^{\prime}(0)\right]$ e $\bar{J}_{i}^{\prime}(0)=$ $\bar{E}_{i}(0)$ para $i=1,2$. Um candidato tensor de Jacobi $\bar{A}$ satisfazendo as condições iniciais $\bar{A}(0)=0, \bar{A}^{\prime}(0)=\mathbb{1}$ pode ser definido requerendo que

$$
\bar{J}_{i}=\bar{A}\left(\bar{E}_{i}\right) \quad i=1,2
$$

e estendendo-o a todo $\tilde{T}_{\gamma(\lambda)} M / \operatorname{span}\left(\gamma^{\prime}(\lambda)\right)$ por linearidade. Como os $\bar{J}_{i}$ 's são classes de Jacobi e os $\bar{E}_{i}$ 's são classes paralelas em $\mathfrak{X}_{q}(\gamma)$, segue que $\bar{A}$ satisfaz (2.11).

Para checar que $\bar{A}$ satisfaz (2.12), vamos supor que $\bar{A}(\lambda)(\bar{V})=\bar{A}^{\prime}(\lambda)(\bar{V})=$ $\left[\gamma^{\prime}(\lambda)\right]$ para algum $\bar{V} \in \tilde{T}_{\gamma(\lambda)} M / \operatorname{span}\left(\gamma^{\prime}(\lambda)\right)$. Como $\tilde{T}_{\gamma(\lambda)} M=\tilde{T}_{\gamma(\lambda)} M / \operatorname{span}\left(\gamma^{\prime}(\lambda)\right) \oplus$ $\operatorname{span}\left(\gamma^{\prime}(\lambda)\right)$, escolhemos o único $V \in \tilde{T}_{\gamma(\lambda)} M$ tal que $[V]=\bar{V}$ e que se escreve como $V=\sum_{i=1}^{2} \alpha_{i} E_{i}$. Daí segue que $\bar{V}=\sum_{i=1}^{2} \alpha_{i} \bar{E}_{i}(\lambda)$ e $\left[\gamma^{\prime}(\lambda)\right]=\bar{A}(\bar{V})=$ $\sum_{i=1}^{2} \alpha_{i} \bar{J}_{i}(\lambda)$. Também

$$
\left[\gamma^{\prime}(\lambda)\right]=\bar{A}^{\prime}(\bar{V})=\sum_{i=1}^{2} \alpha_{i} \bar{A}^{\prime}\left(\bar{E}_{i}\right)=\sum_{i=1}^{2} \alpha_{i} \bar{J}_{i}^{\prime}(\lambda) .
$$

Do fato que $\bar{J}=\sum_{i=1}^{2} \alpha_{i} \bar{J}_{i}$ é uma classe de Jacobi em $\tilde{T}_{\gamma(\lambda)} M / \operatorname{span}\left(\gamma^{\prime}(\lambda)\right)$ com $\bar{J}(\lambda)=\bar{J}^{\prime}(\lambda)=\left[\gamma^{\prime}(\lambda)\right]$, segue que $\bar{J}=\left[\gamma^{\prime}\right]$. Assim, $\sum_{i=1}^{2} \alpha_{i} \bar{E}_{i}(s)=\left[\gamma^{\prime}(s)\right]$ para todo $s \in[0, a]$, contradizendo a hipótese de independência linear das classes paralelas $\bar{E}_{1}, \bar{E}_{2}$. Conclui-se, portanto, que $\operatorname{ker}(\bar{A}(s)) \cap \operatorname{ker}\left(\bar{A}^{\prime}(s)\right)=\left[\gamma^{\prime}(s)\right]$ para todo $s \in[0, a]$.

Vamos resumir o resultado dessa construção na seguinte proposição: 
44 Evolução de feixes de geodésicas nulas e lentes gravitacionais

Proposição 2.6 Sejam $\bar{J}_{1}, \bar{J}_{2}$ classes de Jacobi satisfazendo $\bar{J}_{i}(0)=\left[\gamma^{\prime}(0)\right]$, $\bar{J}_{i}^{\prime}(0)=\left[E_{i}\right](0), i=1,2$ e $\bar{A}$ um operador

$$
\bar{A}: \tilde{T}_{\gamma(\lambda)} M / \operatorname{span}\left(\gamma^{\prime}(\lambda)\right) \rightarrow \tilde{T}_{\gamma(\lambda)} M / \operatorname{span}\left(\gamma^{\prime}(\lambda)\right)
$$

satisfazendo $\bar{A}(0)=0$ e $\bar{A}^{\prime}(0)=\mathbb{1}$ e tal que

$$
\bar{J}_{i}=\bar{A}\left(\bar{E}_{i}\right) \quad i=1,2 .
$$

Então $\bar{A}$ é um tensor de Jacobi.

\subsubsection{Tensores de Lagrange}

Façamos agora alguns comentários sobre a adjunção: embora $\bar{R}=\bar{R}^{*}$ e $\left(\bar{A}^{\prime}\right)^{*}=$ $\left(\bar{A}^{*}\right)^{\prime}$, a adjunta $\bar{A}^{*}$ de um tensor de Jacobi $\bar{A}$ não é necessariamente um tensor de Jacobi uma vez que $(\bar{R} \bar{A})^{*} \neq \bar{R} \bar{A}^{*}$ em geral - na verdade $(\bar{R} \bar{A})^{*}=\bar{A}^{*} \bar{R}$. Isso leva-nos a definir o seguinte objeto:

Definição 2.7 Sejam $\bar{A}$ e $\bar{B}$ dois tensores de Jacobi ao longo de $\gamma$ em $\tilde{T}_{\gamma(\lambda)} M / \operatorname{span}\left(\gamma^{\prime}(\lambda)\right)$. Então seu Wronskiano $W(\bar{A}, \bar{B})$ é o campo tensorial $(1,1)$ em $\tilde{T}_{\gamma(\lambda)} M / \operatorname{span}\left(\gamma^{\prime}(\lambda)\right)$ dado por

$$
W(\bar{A}, \bar{B})=\left(\bar{A}^{\prime}\right)^{*} \bar{B}-\bar{A}^{*} \bar{B}^{\prime} .
$$

Segue do fato que $\bar{R}^{*}=\bar{R}$ e de (2.11) que se $\bar{A}$ e $\bar{B}$ são duas classes de Jacobi em $\tilde{T}_{\gamma(\lambda)} M / \operatorname{span}\left(\gamma^{\prime}(\lambda)\right)$, então $(W(\bar{A}, \bar{B}))^{\prime}=0$. Assim $W(\bar{A}, \bar{B})$ é um campo tensorial constante.

Podemos agora definir campos tensoriais de Lagrange:

Definição 2.8 Um tensor de Jacobi $\bar{A}$ em $\tilde{T}_{\gamma(\lambda)} M / \operatorname{span}\left(\gamma^{\prime}(\lambda)\right)$ é um campo tensorial de Lagrange se $W(\bar{A}, \bar{A})=0$.

O seguinte lema mostra uma importante propriedade de campos de Lagrange:

Lema 2.9 Seja $\bar{A}$ uma classe de Jacobi em $\tilde{T}_{\gamma(\lambda)} M / \operatorname{span}\left(\gamma^{\prime}(\lambda)\right)$. Se $\bar{A}\left(s_{0}\right)=0$ para algum $s_{0} \in[0, a]$, então $\bar{A}$ é um tensor de Lagrange e, em particular,

$$
\left(\bar{A}^{\prime}\right)^{*} \bar{A}=\bar{A}^{*} \bar{A}^{\prime} .
$$




\section{Demonstração}

Sabemos que $W(\bar{A}, \bar{A})$ é um campo de tensores constante. Se $\bar{A}\left(s_{0}\right)=0$, $\bar{A}^{*}\left(s_{0}\right)=0$ e $W(\bar{A}, \bar{A})\left(s_{0}\right)=0$. Assim, $W(\bar{A}, \bar{A})=0$ como pretendíamos.

\subsection{Equação de Raychaudhuri para vetores nulos, condições de energia e pontos conjugados}

Definição 2.10 Seja $\bar{A}$ um tensor de Jacobi ao longo de uma geodésica nula

$\gamma$. Consideremos $\bar{B}=\bar{A}^{\prime} \bar{A}^{-1}$ nos pontos em que $\bar{A}^{-1}$ é definida. Então:

(a) A expansão $\bar{\Theta}$ é definida por

$$
\bar{\Theta}=\operatorname{Tr}(\bar{B})
$$

(b) $O$ tensor vorticidade $\bar{\omega}$ é definido como

$$
\bar{\omega}=\frac{1}{2}\left(\bar{B}-\bar{B}^{*}\right)
$$

(c) O tensor de cisalhamento $\bar{\sigma}$ é definido como

$$
\bar{\sigma}=\frac{1}{2}\left(\bar{B}+\bar{B}^{*}\right)-\frac{\bar{\Theta}}{2} \mathbb{1}
$$

Pode-se mostrar que ${ }^{1}$

$$
\bar{\Theta}=(\operatorname{det} \bar{A})^{-1}(\operatorname{det} \bar{A})^{\prime} .
$$

Com o intuito de calcular o traço $\operatorname{Tr}(\bar{B})^{\prime}$, consideremos:

$$
\bar{B}^{\prime}=\left(\bar{A}^{\prime} \bar{A}^{-1}\right)^{\prime}=\bar{A}^{\prime \prime} \bar{A}^{-1}-\bar{A}^{\prime} \bar{A}^{-1} \bar{A}^{\prime} \bar{A}^{-1}=-\bar{R}-\bar{B} \bar{B} .
$$

${ }^{1}$ Com efeito, seja $A(\lambda) \in \operatorname{Mat}(\mathbb{R}, n)$ uma matriz que depende de forma diferenciável da variável $\lambda$ e que seja invertível (em domínio conveniente), então [42]:

$$
\frac{1}{\operatorname{det} A(\lambda)} \frac{d}{d \lambda} \operatorname{det} A(\lambda)=\operatorname{Tr}\left(A(\lambda)^{-1} \frac{d}{d \lambda} A(\lambda)\right) .
$$


46 Evolução de feixes de geodésicas nulas e lentes gravitacionais

Segue então que

$$
\begin{aligned}
\bar{\Theta}^{\prime} & =\operatorname{Tr}\left(\bar{B}^{\prime}\right)=-\operatorname{Tr}(\bar{R})-\operatorname{Tr}(\bar{B} \bar{B}) \\
& =-\operatorname{Tr}(\bar{R})-\operatorname{Tr}\left(\left(\bar{\omega}+\bar{\sigma}+\frac{\bar{\Theta}}{2} \mathbb{1}\right)^{2}\right) \\
& =-\operatorname{Tr}(\bar{R})-\operatorname{Tr}\left(\bar{\omega}^{2}\right)-\operatorname{Tr}\left(\bar{\sigma}^{2}\right)-\frac{\bar{\Theta}^{2}}{2}
\end{aligned}
$$

pois $\operatorname{Tr}(\bar{\omega})=\operatorname{Tr}(\bar{\sigma})=\operatorname{Tr}(\bar{\omega} \bar{\sigma})=0$.

Devemos calcular o traço de $\bar{R}$. Para tanto, sejam $\left\{E_{1}, E_{2}\right\}$ uma base ortonormal para $\tilde{T}_{p} M / \operatorname{span}(k)$ em todo ponto $p=\gamma(\lambda)$. Vamos estender $\left\{E_{1}, E_{2}\right\}$ a uma base ortonormal $\left\{E_{1}, E_{2}, E_{3}, E_{4}\right\}$ ao longo de $\gamma$, onde $E_{4}$ é tipo-tempo e $\gamma^{\prime}=\left(E_{3}+E_{4}\right) / \sqrt{2}$. Temos, usando a Proposição A.22,

$$
\left\langle R\left(E_{3}, \gamma^{\prime}\right) \gamma^{\prime}, E_{3}\right\rangle-\left\langle R\left(E_{4}, \gamma^{\prime}\right) \gamma^{\prime} a, E_{4}\right\rangle=0
$$

e, em conseqüência deste fato, obtemos

$$
\begin{aligned}
\operatorname{Tr}(\bar{R}) & =\sum_{i=1}^{2}\left\langle\bar{R}\left(\left[E_{i}\right], \gamma^{\prime}\right) \gamma^{\prime},\left[E_{i}\right]\right\rangle_{q} \\
& =\sum_{i=1}^{2}\left\langle R\left(E_{i}, \gamma^{\prime}\right) \gamma^{\prime}, E_{i}\right\rangle \\
& =\sum_{i=1}^{4}\left\langle E_{i}, E_{i}\right\rangle\left\langle R\left(E_{i}, \gamma^{\prime}\right) \gamma^{\prime}, E_{i}\right\rangle=\operatorname{Ric}\left(\gamma^{\prime}, \gamma^{\prime}\right)
\end{aligned}
$$

Completado este cálculo temos reunidos todos os ingredientes para escrever a equação de Raychaudhuri para tensores de Jacobi ao longo de geodésicas nulas:

$$
\bar{\Theta}^{\prime}=-\operatorname{Ric}\left(\gamma^{\prime}, \gamma^{\prime}\right)-\operatorname{Tr}\left(\bar{\omega}^{2}\right)-\operatorname{Tr}\left(\bar{\sigma}^{2}\right)-\frac{\bar{\Theta}^{2}}{2} .
$$

Lema 2.11 Se $\bar{A}$ é um campo de tensores de Lagrange, então o tensor de vorticidade $\bar{\omega}$ anula-se sobre $\gamma$.

\section{Demonstração}

A equação $\bar{A}^{\prime *} \bar{A}-\bar{A}^{*} \bar{A}^{\prime}=0$ implica que $\bar{B}=\left(\bar{A}^{*}\right)^{-1}\left(\bar{A}^{*}\right)^{\prime} \bar{A} \bar{A}^{-1}=\left(\bar{A}^{*}\right)^{-1}\left(\bar{A}^{*}\right)^{\prime}=$ $\bar{B}^{*}$, ou seja, que $\bar{B}$ é auto-adjunto.

Desta forma obtivemos a equação de Raychaudhuri livre de vorticidade para campos de Lagrange ao longo de geodésicas nulas: 


$$
\bar{\Theta}^{\prime}=-\operatorname{Ric}\left(\gamma^{\prime}, \gamma^{\prime}\right)-\operatorname{Tr}\left(\bar{\sigma}^{2}\right)-\frac{\bar{\Theta}^{2}}{2} .
$$

A equação de Raychaudhuri desempenha importante papel na demonstração de teoremas de singularidade de Hawking-Penrose [15] justamente porque fornece métodos para determinar condições suficientes para a existência de pontos conjugados ao longo de geodésicas. As proposições 4.4.4 e 4.4.5 de [15] condensam estas condições. Por conveniência as transcreveremos aqui:

Proposição 2.12. (proposição 4.4.4 de [15]) Se $R_{\mu \nu} k^{\mu} k^{\nu} \geq 0$ em toda a parte e se em algum ponto $\gamma\left(\lambda_{1}\right)$ ao longo de uma geodésica nula $\gamma:[0, a] \rightarrow M$ a taxa de expansão $\bar{\Theta}_{1}$ é negativa, então existirá um ponto conjugado a $\gamma(0)$ ao longo de $\gamma(\lambda)$ entre $\gamma\left(\lambda_{1}\right)$ e $\gamma\left(\lambda_{1}+\left(2 /\left[-\bar{\Theta}_{1}\right)\right]\right)$, contanto que $\gamma(\lambda)$ possa ser estendida a este intervalo.

Proposição 2.13. (proposição 4.4.5 de [15]) Se $R_{\mu \nu} k^{\mu} k^{\nu} \geq 0$ em toda a parte e se para algum $p=\gamma\left(\lambda_{1}\right), k^{\mu} k^{\nu} k_{[\alpha} R_{\beta] \mu \nu[\rho} k_{\eta]}$ é não-nulo, então existirão $\lambda_{0}$ e $\lambda_{2}$ tais que $q=\gamma\left(\lambda_{0}\right)$ e $r=\gamma\left(\lambda_{2}\right)$ serão conjugados ao longo de $\gamma$ contanto que $\gamma(\lambda)$ possa ser estendida a estes valores.

A condição de que $k^{\mu} k^{\nu} k_{[\alpha} R_{\beta] \mu \nu[\rho} k_{\eta]}$ seja não-nulo em algum ponto sobre toda geodésica nula definida no espaço-tempo é conhecida como condição genérica e pode ser refraseada de uma maneira mais geométrica como o requerimento de que exista algum $\lambda_{0}$ no domínio de definição da geodésica nula $\gamma$ para o qual o endomorfismo da curvatura

$$
\bar{R}\left(., \gamma^{\prime}\left(\lambda_{0}\right)\right) \gamma^{\prime}\left(\lambda_{0}\right): \tilde{T}_{\gamma(\lambda)} M / \operatorname{span}\left(\gamma^{\prime}(\lambda)\right) \rightarrow \tilde{T}_{\gamma(\lambda)} M / \operatorname{span}\left(\gamma^{\prime}(\lambda)\right)
$$

com $p=\gamma\left(\lambda_{0}\right)$ não seja identicamente zero para toda geodésica nula [9].

A condição de que $R_{\mu \nu} k^{\mu} k^{\nu} \geq 0$ para todo vetor nulo $k \in T M$ é conhecida como condição forte de energia.

No contexto de lentes gravitacionais estas proposições mostram que, em circunstâncias bastante genéricas, são satisfeitas condições para que existam pontos conjugados ao longo de geodésicas nulas emergindo de uma fonte, contanto que se possa prolongar o suficiente as geodésicas. Este resultado pode ser traduzido na afirmação de que toda lente gravitacional gerada por distribuições de matéria satisfazendo condições de energia forte e genérica é uma lente forte, no sentido de que sempre existirão imagens múltiplas da fonte se as geodésicas (diretas no futuro) puderem ser suficientemente prolongadas. 
48 Evolução de feixes de geodésicas nulas e lentes gravitacionais

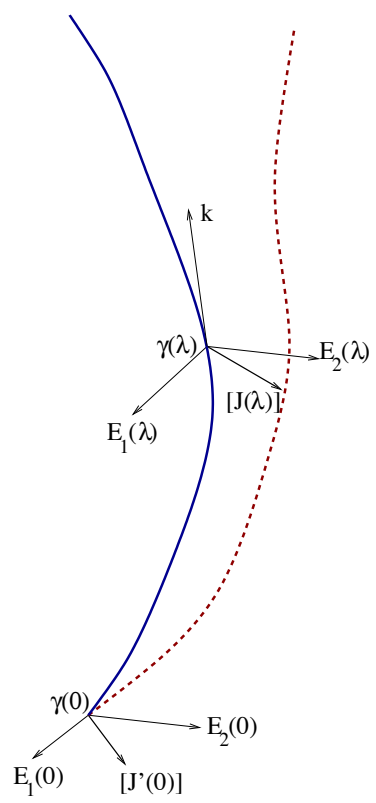

Fig. 2.1 Descrição da separação em direções tipo-espaço de elementos de um feixe de geodésicas nulas. A classe de Jacobi descreve o afastamento de uma dada geodésica do feixe em relação à geodésica fiducial. A velocidade inicial de afastamento entre as curvas é a variável determinante do comportamento.

\subsection{Equações centrais da teoria de Lentes Gravitacionais}

\subsubsection{Decomposição de classes de Jacobi em uma base ortonormal}

Aprendemos do Lema 2.2 que cada campo de Jacobi $J$ dado em $\tilde{T}_{\gamma(\lambda)} M$ dá origem a uma classe de Jacobi $[J]$ em $\tilde{T}_{\gamma(\lambda)} M / \operatorname{span}\left(\gamma^{\prime}(\lambda)\right)$. Sabemos também que neste espaço e ao longo da geodésica $\gamma$ existe uma base ortonormal de vetores tipo-espaço $\left\{\bar{E}_{1}, \bar{E}_{2}\right\}$ paralelamente propagada e que restrita a $\tilde{T}_{\gamma(\lambda)} M / \operatorname{span}\left(\gamma^{\prime}(\lambda)\right)$ a métrica $\langle., .\rangle_{q}$ é positiva-definida. Podemos, então, definir a decomposição de $[J]$ como

$$
[J]=\xi_{1} \bar{E}_{1}+\xi_{2} \bar{E}_{2}
$$

com

$$
\xi_{i}=\left\langle[J], \bar{E}_{i}\right\rangle_{q} \quad i=1,2 .
$$

A decomposição da classe de Jacobi em termos da base nas classes de equivalência é ilustrada na Figura 2.1. 
Como sintetizado na Proposição 2.6, é possível obter construtivamente um tensor de Jacobi caso classes de Jacobi linearmente independentes em $\tilde{T}_{\gamma(\lambda)} M / \operatorname{span}\left(\gamma^{\prime}(\lambda)\right)$ forem dadas ao longo de $\gamma$, satisfazendo as condições iniciais $\bar{J}_{i}(0)=\left[\gamma^{\prime}(0)\right]$ e $\bar{J}_{i}^{\prime}(0)=\bar{E}_{i}(0), i=1,2$. Neste caso, um tensor de Jacobi $\bar{A}$ satisfazendo as condições $\bar{A}(0)=0, \bar{A}^{\prime}(0)=\mathbb{1}$ é definido requerendo que $\bar{J}_{i}=\bar{A}\left(\bar{E}_{i}\right), i=1,2$ e então estendido a todo $\tilde{T}_{p} M / \operatorname{span}(k)$ por linearidade. Notamos também que dada a condição inicial $\bar{A}(0)=0$ satisfeita por tensores de Jacobi obtidos através desta construção, o Lema 2.9 estabelece que o tensor de Jacobi $\bar{A}$ é também um tensor de Lagrange.

Realizemos explicitamente uma construção desse tipo nas seções que seguem.

\subsubsection{Forma explícita do endomorfismo da curvatura no espaço quociente}

Obteremos a maneira explícita como o endomorfismo $\bar{R}\left(., \gamma^{\prime}\right) \gamma^{\prime}$ atua sobre vetores em $\tilde{T}_{\gamma(\lambda)} M / \operatorname{span}\left(\gamma^{\prime}(\lambda)\right)$. Para tanto, consideremos $\epsilon=\bar{E}_{1}+i \bar{E}_{2}, \bar{\epsilon}=$ $\bar{E}_{1}-i \bar{E}_{2}^{2}$ e $\xi=\xi_{1}+i \xi_{2}$. Dada (2.26) e relembrando que $\bar{E}_{i}$ são transportados paralelamente ao longo de $\gamma$, podemos escrever

$$
\begin{aligned}
\xi^{\prime \prime} & =\left\langle\epsilon,[J]^{\prime \prime}\right\rangle_{q}=-\left\langle\epsilon, \bar{R}\left([J], \gamma^{\prime}\right) \gamma^{\prime}\right\rangle_{q} \\
& =-\left\langle\epsilon, \bar{R}\left(\frac{1}{2} \xi \bar{\epsilon}+\frac{1}{2} \bar{\xi} \epsilon, \gamma^{\prime}\right) \gamma^{\prime}\right\rangle_{q} \\
& =-\xi \underbrace{\frac{1}{2}\left\langle\epsilon, \bar{R}\left(\bar{\epsilon}, \gamma^{\prime}\right) \gamma^{\prime}\right\rangle_{q}}_{\mathcal{R}}-\bar{\xi} \underbrace{\frac{1}{2}\langle\epsilon, \bar{R}(\epsilon, k) k\rangle_{q}}_{\mathcal{F}} \\
& =-\mathcal{R} \xi-\mathcal{F} \bar{\xi}
\end{aligned}
$$

onde usamos (2.8), (2.6) e (2.7) para estabelecer as igualdades da primeira linha.

Caracterizemos $\mathcal{R}$ e $\mathcal{F}$ :

Proposição 2.14 Os objetos $\mathcal{R}$ e $\mathcal{F}$ podem ser expressos como $\mathcal{R}=\frac{1}{2} R_{\mu \nu} k^{\mu} k^{\nu}$ e $\mathcal{F}=\frac{1}{2} \epsilon^{\alpha} \epsilon^{\beta} C_{\alpha \mu \beta \nu} k^{\mu} k^{\nu}$.

\footnotetext{
${ }^{2}$ Notando que por infelicidade de notação utilizamos a barra sobrescrita para indicar conjugação complexa e a restrição de vetores ao espaço quociente, mas isto não deve ser obstáculo para a compreensão.
} 
50 Evolução de feixes de geodésicas nulas e lentes gravitacionais

Demonstração:

$$
\begin{aligned}
\mathcal{R} & =\frac{1}{2}\left\langle\epsilon, R\left(\bar{\epsilon}, \gamma^{\prime}\right) \gamma^{\prime}\right\rangle=\frac{1}{2} \bar{\epsilon}^{\alpha} \epsilon^{\beta} R_{\alpha \mu \beta \nu} k^{\mu} k^{\nu} \\
& =\frac{1}{4}\left(\bar{\epsilon}^{\alpha} \epsilon^{\beta}+\epsilon^{\alpha} \bar{\epsilon}^{\beta}\right) R_{\alpha \mu \beta \nu} k^{\mu} k^{\nu}=\frac{1}{2} g^{\alpha \beta} R_{\alpha \mu \beta \nu} k^{\mu} k^{\nu}=\frac{1}{2} R_{\mu \nu} k^{\mu} k^{\nu}
\end{aligned}
$$

De acordo com a definição, $\mathcal{F}=\frac{1}{2} \epsilon^{\alpha} \epsilon^{\beta} R_{\alpha \mu \beta \nu} k^{\mu} k^{\nu}$. Podemos reescrever as componentes do tensor de Riemann decomposto em termos do tensor de Weyl (A.11):

$$
R_{\alpha \mu \beta \nu}=C_{\alpha \mu \beta \nu}+\frac{2}{n-2}\left(g_{\alpha[\beta} R_{\nu] \mu}-g_{\mu[\beta} R_{\nu] \alpha}\right)-\frac{2}{(n-1)(n-2)} R g_{\alpha[\beta} g_{\nu] \mu}
$$

Notando que, por definição, $\langle k, k\rangle=\langle\epsilon, \epsilon\rangle=\langle\epsilon, k\rangle=0$, teremos que, para $n=4 \mathrm{em}$ (A.11)

$$
\epsilon^{\alpha} \epsilon^{\beta} R_{\alpha \mu \beta \nu} k^{\mu} k^{\nu}=\epsilon^{\alpha} \epsilon^{\beta} C_{\alpha \mu \beta \nu} k^{\mu} k^{\nu}
$$

e, portanto, $\mathcal{F}=\frac{1}{2} \epsilon^{\alpha} \epsilon^{\beta} C_{\alpha \mu \beta \nu} k^{\mu} k^{\nu}$ como pretendíamos.

Escrevendo novamente as componentes de $\xi$ como um vetor, podemos reescrever o sistema obtido como

$$
\boldsymbol{\xi}^{\prime \prime}+T \boldsymbol{\xi}=0
$$

onde $T$ é chamada de matriz de maré ótica ${ }^{3}$ na literatura de lentes gravitacionais, dada explicitamente por:

$$
T=\left(\begin{array}{cc}
\mathcal{R}+\operatorname{Re} \mathcal{F} & \operatorname{Im} \mathcal{F} \\
\operatorname{Im} \mathcal{F} & \mathcal{R}-\operatorname{Re} \mathcal{F}
\end{array}\right)
$$

Reconhecemos, com efeito, que $(2.32)$ é a forma matricial de $\bar{R}\left(., \gamma^{\prime}\right) \gamma^{\prime}$ atuando em elementos de $\tilde{T}_{\gamma(\lambda)} M / \operatorname{span}\left(\gamma^{\prime}(\lambda)\right)$.

\subsubsection{Aplicação de Jacobi}

Vamos agora empregar propriedades de campos de Jacobi para obter o que se denomina aplicação de Jacobi. Como notamos na Proposição A.28, um campo de Jacobi satisfazendo $J(0)=0$ é da forma

$$
J(\lambda)=\left(\operatorname{dexp}_{p}\right)_{\lambda k}(\lambda w)
$$

sendo $w$ a velocidade de afastamento das geodésicas em $\lambda=0, k=\gamma^{\prime}(0)$.

${ }^{3}$ Optical tidal matrix em inglês. 
Se escrevermos $J(\lambda)=D(\lambda) w:=\left[\lambda\left(d_{\exp _{p}}\right)_{\lambda k}\right] w$, notamos que a transformação linear $D(\lambda)$ deve satisfazer, em conseqüência de (A.27) $D(0)=0$ e $D^{\prime}(0)=\mathbb{1}$. Interessamo-nos por escrever uma tal transformação no conjunto das classes de equivalência. Para tanto, definimos em $\tilde{T}_{\gamma(\lambda)} M$ a decomposição de $J^{\prime}(0)$ como $J^{\prime}(0)=\theta_{1} E_{1}(0)+\theta_{2} E_{2}(0)+\alpha k$ e notamos que

$$
\xi_{i}(\lambda)=\left\langle[J], \bar{E}_{i}\right\rangle_{q}(\lambda)=\left\langle J, E_{i}\right\rangle(\lambda)=\left\langle\lambda\left(\operatorname{dexp}_{p}\right)_{\lambda k}\left(\theta_{1} E_{1}+\theta_{2} E_{2}\right)(0), E_{i}(\lambda)\right\rangle
$$

já que $\left\langle\lambda\left(\operatorname{dexp}_{p}\right)_{\lambda k} k, E_{i}\right\rangle=0$.

Devemos notar que $\left\langle\left[\lambda\left(\operatorname{dexp}_{p}\right)_{\lambda k} .\right],[.]\right\rangle_{q}$ constitui uma forma bilinear em $\tilde{T}_{\gamma(\lambda)} M / \operatorname{span}\left(\gamma^{\prime}(\lambda)\right)$ e, portanto, admite uma representação matricial em termos de sua ação em vetores de base. Assim, podemos escrever

$$
\left(\begin{array}{l}
\xi_{1} \\
\xi_{2}
\end{array}\right)=\left(\begin{array}{l}
\left\langle\lambda\left(\operatorname{dexp}_{p}\right)_{\lambda k} E_{1}(0), E_{1}(\lambda)\right\rangle\left\langle\lambda\left(d \exp _{p}\right)_{\lambda k} E_{1}(0), E_{2}(\lambda)\right\rangle \\
\left\langle\lambda\left(\operatorname{dexp}_{p}\right)_{\lambda k} E_{2}(0), E_{1}(\lambda)\right\rangle\left\langle\lambda\left(\operatorname{dexp}_{p}\right)_{\lambda k} E_{2}(0), E_{2}(\lambda)\right\rangle
\end{array}\right)\left(\begin{array}{c}
\theta_{1} \\
\theta_{2}
\end{array}\right) .
$$

Obtivemos (2.33) seguindo o procedimento construtivo estabelecido para obtermos um tensor de Jacobi a partir de classes de vetores linearmente independentes em $\mathfrak{X}_{q}\left(\tilde{T}_{\gamma(\lambda)} M / \operatorname{span}\left(\gamma^{\prime}(\lambda)\right)\right)$. Concluímos, portanto, que (2.33) equivale a termos obtidos a forma matricial de $\bar{A}$ para que $\bar{J}_{i}(\lambda)=\bar{A}(\lambda)\left(\bar{E}_{i}(0)\right)$ seja satisfeita.

Com o intuito de aproximar nossa notação à usualmente empregada na literatura de lentes gravitacionais, definimos $\boldsymbol{\theta}:=\left[J^{\prime}(0)\right]$. Com isso, escrevemos

$$
\boldsymbol{\xi}=\mathcal{D}(\lambda) \boldsymbol{\theta} .
$$

Esta aplicação é chamada de mapa de Jacobi. Facilmente observa-se que a matriz $\mathcal{D}$ satisfaz $\mathcal{D}(0)=0$ e $\mathcal{D}^{\prime}(0)=\mathbb{1}$.

Inserindo (2.34) em (2.31), obtemos

$$
\mathcal{D}^{\prime \prime}(\lambda)+T \mathcal{D}=0,
$$

que justamente é equivalente à equação (2.11) se recobramos que $T$ é, com efeito, equivalente a escrevermos $\bar{R}$.

Para mostrar que $\mathcal{D}(\lambda)$ é um tensor de Jacobi em $\tilde{T}_{\gamma(\lambda)} M / \operatorname{span}\left(\gamma^{\prime}(\lambda)\right)$ restanos ainda verificar que

$$
\operatorname{ker}(\mathcal{D}(\lambda)) \cap \operatorname{ker}\left(\mathcal{D}^{\prime}(\lambda)\right)=\left[\gamma^{\prime}(\lambda)\right]
$$

para todos os valores de $\lambda$ para os quais $\gamma$ é definida. Vamos, então calcular $\mathcal{D}^{\prime}(\lambda)$ para $\lambda \neq 0$. Usaremos para tanto a identidade (A.26) e o fato de $E_{i}(\lambda)$, $i=1,2$ serem paralelamente transportados ao longo de $\gamma$. Explicitamente, 
52 Evolução de feixes de geodésicas nulas e lentes gravitacionais

$$
\begin{aligned}
\mathcal{D}^{\prime}(\lambda)= & \left(\begin{array}{l}
\left\langle\left(d \exp _{p}\right)_{\lambda k} E_{1}(0), E_{1}(\lambda)\right\rangle\left\langle\left(d \exp _{p}\right)_{\lambda k} E_{1}(0), E_{2}(\lambda)\right\rangle \\
\left\langle\left(d \exp _{p}\right)_{\lambda k} E_{2}(0), E_{1}(\lambda)\right\rangle\left\langle\left(d \exp _{p}\right)_{\lambda k} E_{2}(0), E_{2}(\lambda)\right\rangle
\end{array}\right) \\
& +\lambda \frac{d}{d \lambda}\left(\begin{array}{c}
\left\langle\left(d \exp _{p}\right)_{\lambda k} E_{1}(0), E_{1}(\lambda)\right\rangle\left\langle\left(d \exp _{p}\right)_{\lambda k} E_{1}(0), E_{2}(\lambda)\right\rangle \\
\left\langle\left(d \exp _{p}\right)_{\lambda k} E_{2}(0), E_{1}(\lambda)\right\rangle\left\langle\left(d \exp _{p}\right)_{\lambda k} E_{2}(0), E_{2}(\lambda)\right\rangle
\end{array}\right) .
\end{aligned}
$$

Para determinar se há elementos comuns ao núcleo de $\mathcal{D}$ e $\mathcal{D}^{\prime}$, vamos averiguar se elementos do núcleo de $\mathcal{D}$ podem estar no núcleo de $\mathcal{D}^{\prime}$. Observamos de $(2.33)$ que, para $\lambda \neq 0, \mathcal{D}$ só é singular em pontos conjugados. Mas em pontos conjugados o primeiro termo do lado direito de (2.36) é também singular. O segundo termo, entretanto, não pode ser singular pois envolve a derivada de campos de Jacobi nas vizinhanças de um ponto conjugado e sabe-se que, como corolário do Teorema do Índice de Morse, pontos conjugados ao longo de geodésicas nulas são isolados. Sabemos também que para $\lambda=0, \mathcal{D}^{\prime}(0)=\mathbb{1}$ e portanto para nenhum valor do parâmetro há elementos comuns aos núcleos, exceto elementos da classe de equivalência $\left[\gamma^{\prime}(\lambda)\right]$. Conclui-se, portanto, que elementos não triviais do núcleo de $\mathcal{D}$ não podem ser comuns ao núcleo de $\mathcal{D}^{\prime}$ e consequentemente $\mathcal{D}$ satisfaz as duas condições requeridas para ser um tensor de Jacobi. Além disso, como $\mathcal{D}(0)=0$, o tensor de Jacobi construído também é um tensor de Lagrange.

Estudar as soluções de (2.35) sujeita às condições iniciais introduzidas permitenos descrever a evolução de um feixe de geodésicas sob o efeito da curvatura, e por isso constitui a equação central no estudo de lentes gravitacionais. Ao obter as relações (2.34) e (2.35) cumprimos nosso objetivo de substituir a derivação apresentada por [1] dessas equações.

\subsection{Aplicação: O mapa de Jacobi no caso cosmológico}

Trabalharemos com a hipótese de que os potenciais gravitacionais cosmológicos são apenas capazes de produzir efeitos típicos de lentes fracas, ou seja, assumiremos que o cone de luz passado dos eventos de observação sejam subvariedades mergulhadas do espaço-tempo. Assumiremos um espaço de FLRW e que entre as geodésicas nulas desse espaço aqui consideradas, tenhamos $\gamma(0)=p$ como o evento de observação, que sejam direcionadas ao passado e parametrizadas por um parâmetro afim $\lambda$, como de costume.

Revisando nosso programa, queremos resolver a equação:

$$
\mathcal{D}^{\prime \prime}(\lambda)+T \mathcal{D}=0
$$


sujeitas às condições iniciais $\mathcal{D}(0)=0$ e $\dot{\mathcal{D}}(0)=\mathbb{1}$, com $T$ dada por

$$
T=\left(\begin{array}{cc}
\mathcal{R}+\operatorname{Re} \mathcal{F} & \operatorname{Im} \mathcal{F} \\
\operatorname{Im} \mathcal{F} & \mathcal{R}-\operatorname{Re} \mathcal{F}
\end{array}\right)
$$

sendo suas entradas relacionadas a quantidades geométricas $\mathcal{R}=\frac{1}{2} R_{\mu \nu} k^{\mu} k^{\nu}$ e $\mathcal{F}=\frac{1}{2} \epsilon^{\alpha} \epsilon^{\beta} C_{\alpha \mu \beta \nu} k^{\mu} k^{\nu}$ a serem calculadas no espaço-tempo de interesse, que para nós é o espaço FLRW sujeito a perturbações escalares e parametrizado (no gauge newtoniano) como:

$$
g=a^{2}(\eta)\left[-(1+2 \Phi) d^{2} \eta+(1-2 \Psi)^{(3)} g\right]
$$

onde $\Phi$ e $\Psi$ são os potenciais gravitacionais, também chamados de potenciais de Bardeen e $\eta$ é o tempo conforme.

A equação (2.37), que é uma equação de segunda ordem, pode ser escrita como um sistema de equações de primeira ordem. Definindo

$$
\mathcal{Y}=\frac{d \mathcal{D}}{d \lambda}
$$

temos

$$
\frac{d}{d \lambda}\left(\begin{array}{l}
\mathcal{D} \\
\mathcal{Y}
\end{array}\right)=-\left(\begin{array}{cc}
0 & -1 \\
T(\lambda) & 0
\end{array}\right)\left(\begin{array}{l}
\mathcal{D} \\
\mathcal{Y}
\end{array}\right), \quad\left(\begin{array}{l}
\mathcal{D} \\
\mathcal{Y}
\end{array}\right)(0)=\left(\begin{array}{l}
0 \\
1
\end{array}\right)
$$

lembrando que as entradas desta equação são, de fato, matrizes.

Sabemos que se $\Phi=\Psi=0$ em (2.39), podemos invocar o teorema A.26 para determinar a matriz de maré óptica, que neste caso é bastante simples (fazendo $a(\eta) \equiv 1$ ):

$$
T^{(0)}=\left(\begin{array}{cc}
K & 0 \\
0 & K
\end{array}\right)
$$

com $K$ sendo a curvatura seccional. O fato de termos uma matriz tão simples deve-se ao tensor de Weyl anular-se no espaço-tempo base, como conseqüência do Teorema A.26.

Supondo que as correções devidas aos potenciais gravitacionais são "pequenas", podemos escrever

$$
\left(\begin{array}{cc}
0 & -1 \\
T(\lambda) & 0
\end{array}\right)=\underbrace{\left(\begin{array}{cc}
0 & -1 \\
K & 0
\end{array}\right)}_{L}+\underbrace{\left(\begin{array}{cc}
0 & 0 \\
T^{(1)}(\lambda) & 0
\end{array}\right)}_{I(\lambda)}
$$

donde obtivemos um problema de perturbações sobre um sistema linear. A solução de ordem zero será dada por $\mathrm{e}^{L \lambda}$ aplicada à condição inicial. Explici- 
54 Evolução de feixes de geodésicas nulas e lentes gravitacionais

tamente, a solução (para $\mathcal{D}^{(0)}$ ) é dada por ${ }^{4}$

$$
\mathcal{D}^{(0)}(\lambda)= \begin{cases}K^{-1 / 2} \operatorname{sen}(\sqrt{K} \lambda) & K>0 \\ \lambda & K=0 \\ (-K)^{-1 / 2} \operatorname{senh}(\sqrt{-K} \lambda) & K<0\end{cases}
$$

Esta solução corresponde à solução clássica de campos de Jacobi em espaços de curvatura constante [17].

A primeira correção devida a perturbações (e a única que consideraremos) é dada, sendo $I$ definida em (2.42), por:

$$
\mathrm{e}^{L \lambda}\left(\int_{0}^{\lambda} \mathrm{e}^{-L \lambda^{\prime}} I\left(\lambda^{\prime}\right) \mathrm{e}^{L \lambda^{\prime}} d \lambda^{\prime}\right)\left(\begin{array}{l}
0 \\
1
\end{array}\right)
$$

Ao computar esta contribuição, obtemos $\mathcal{D}^{(1)}(\lambda)$ :

$$
\mathcal{D}^{(1)}=-\int_{0}^{\lambda} \mathcal{D}^{(0)}\left(\lambda-\lambda^{\prime}\right) \mathcal{D}^{(0)}\left(\lambda^{\prime}\right) T^{(1)}\left(\lambda^{\prime}\right) d \lambda^{\prime}
$$

ou,

$$
\left(\frac{\mathcal{D}^{(1)}}{\mathcal{D}^{(0)}}\right)=-\int_{0}^{\lambda} \frac{\mathcal{D}^{(0)}\left(\lambda-\lambda^{\prime}\right) \mathcal{D}^{(0)}\left(\lambda^{\prime}\right)}{\mathcal{D}^{(0)}(\lambda)} T^{(1)}\left(\lambda^{\prime}\right) d \lambda^{\prime},
$$

com $T^{(1)}$ calculada sobre $\gamma(\lambda)$.

Para expor finalmente a solução, precisamos explicitar qual a forma exata da inhomogeneidade, ou seja, como escrever as componentes de $T^{(1)}$ em termos dos potenciais que parametrizam as perturbações da métrica.

\subsubsection{Cálculo do tensor de Riemann}

Hipóteses. Escrevemos a métrica do espaço FLRW sujeito a perturbações escalares como (2.39). Para realizar o cálculo do tensor de Riemann tomaremos três simplificações: não tomaremos em conta o fator de escala, consideraremos apenas o caso da métrica FLRW com seções espaciais planas, e consideraremos ainda que as derivadas temporais dos potenciais de Bardeen são infinitésimos de ordem superior.

\footnotetext{
${ }^{4}$ Devemos notar que, como notamos, estamos buscando uma solução matricial. A matriz $\mathcal{D}^{(0)}$, no entanto é dada por uma função multiplicando a matriz identidade e por um abuso de notação denotaremos tal função também por $\mathcal{D}^{(0)}$. Quando quisermos nos referir explicitamente à matriz, escreveremos $\mathcal{D}^{(0)} \mathbb{1}$
} 
A primeira prática significa realizar cálculos com uma métrica mais simples e conforme a (2.39). Neste ponto, no entanto, devemos ser cuidadosos já que o tensor de Riemann não é conformemente invariante, mas o tensor de Weyl, que é a parte do tensor de Riemann que mais nos interessa, o é. Para o cálculo do tensor de Ricci, que também não é conformemente invariante, incorporaremos os fatores conformes no final.

Escolher seções espaciais planas, o que corresponde à segunda suposição, tem novamente o intuito de simplificar as expressões, além de estar em conformidade com os resultados experimentais sobre a curvatura espacial no modelo cosmológico padrão. A justificativa para tomar derivadas temporais dos potenciais de Bardeen como de ordem superior neste cálculo tem justificativa essencialmente ligada ao entendimento atual da dinâmica destes potenciais durante a fase de dominação pela matéria no universo [44].

Cálculos. Consideremos a métrica

$$
g=-(1+2 \Phi) d^{2} \eta+(1-2 \Psi)\left(d^{3} \mathbf{x}\right)^{2} .
$$

Utilizando os métodos descritos no apêndice A.5, identificamos

$$
\hat{\theta}^{0}=(1+\Phi) d \eta \quad \hat{\theta}^{i}=(1-\Psi) d x^{i}
$$

com $i=1,2,3$.

Utilizando a primeira equação de estrutura de Cartan (A.20), notando que temos uma conexão de Levi-Civita e consequentemente torção nula, calculamos as 1-formas de conexão não nulas:

$$
\begin{aligned}
\omega_{i}^{0} & =\Phi_{, i} \hat{\theta}^{0} \\
\omega_{j}^{i} & =\Psi_{i} \hat{\theta}^{j}-\Psi_{j} \hat{\theta}^{i}
\end{aligned}
$$

A segunda equação de estrutura de Cartan (A.21) permite escrever as componentes não nulas do tensor de Riemann:

$$
\begin{aligned}
\frac{1}{2} R_{i 0 j}^{0} & =-\Phi_{, i j} & \frac{1}{2} R_{j i j}^{i} & =\Psi_{, i i}+\Psi_{, j j} \\
\frac{1}{2} R_{213}^{1} & =\Psi_{, 23} & \frac{1}{2} R_{323}^{1}=\Psi_{, 12} & \frac{1}{2} R_{223}^{1}=-\Psi_{, 13} .
\end{aligned}
$$


56 Evolução de feixes de geodésicas nulas e lentes gravitacionais

As componentes do tensor de Ricci não nulas são:

$$
R_{00}=2 \triangle \Phi \quad R_{i j}=\left(\Psi_{, i j}-\Phi_{, i j}\right)+2 \triangle \Psi \eta_{i j} .
$$

O laplaciano é tomado nas dimensões espaciais.

A curvatura escalar é dada por:

$$
R=4 \triangle(\Psi-\Phi)+4 \triangle \Psi
$$

Podemos finalmente determinar as componentes do tensor de Weyl segundo a equação (A.11):

$$
\begin{aligned}
C_{0 i 0 j} & =\left(\Phi_{, i i}+\Psi_{, j j}\right)-\frac{1}{3} \triangle(\Phi+\Psi) \eta_{i j} \\
C_{1213} & =\left(\Phi_{, 23}+\Psi_{, 23}\right) \\
C_{3132} & =\left(\Phi_{, 12}+\Psi_{, 12}\right) \\
C_{2123} & =\left(\Phi_{, 13}+\Psi_{, 13}\right) \\
C_{i j i j} & =\left(\Phi_{, i i}+\Psi_{, i i}\right)+\left(\Phi_{, j j}+\Psi_{, j j}\right)-\frac{2}{3} \triangle(\Phi+\Psi) .
\end{aligned}
$$

Com finalmente todos os ingredientes reunidos, computam-se $\mathcal{R}$ e $\mathcal{F}$ :

$$
\begin{gathered}
\mathcal{R}=\triangle(\Phi+\Psi) \\
\mathcal{F}=\left[\left(\Phi_{, 11}+\Psi_{, 11}\right)-\left(\Phi_{, 22}+\Psi_{, 22}\right)\right]+2 i\left(\Phi_{, 12}+\Psi_{, 12}\right) .
\end{gathered}
$$

Devolução de fatores conformes. Como mencionamos, escolhemos calcular $\mathcal{R}$ e $\mathcal{F}$ em uma métrica sem fator conforme para simplificar cálculos, mas deveríamos no final da conta devolver os fatores conformes devidos. Sabemos que o tensor de Weyl é conformemente invariante mas não são invariantes o tensor de Ricci nem $\gamma^{\prime}(\lambda)$, de onde poderão surgir potências do fator conforme.

Sendo $\tilde{g}=\Omega^{2} g$ e valendo $\frac{d \tilde{\lambda}}{d \lambda}=\Omega^{2}$ sabemos que [41]: $\tilde{R}_{\mu \nu}=R_{\mu \nu}-2 \nabla_{\mu} \nabla_{\nu} \ln \Omega-g_{\mu \nu} g^{\rho \sigma} \nabla_{\rho} \nabla_{\sigma} \ln \Omega+2\left(\nabla_{\mu} \ln \Omega\right) \nabla_{\nu} \ln \Omega-2 g_{\mu \nu} g^{\rho \sigma}\left(\nabla_{\rho} \ln \Omega\right) \nabla_{\sigma} \ln \Omega$.

Daí segue que em nosso caso teremos 


$$
\tilde{\mathcal{R}}=\frac{1}{\Omega^{4}}\left[\mathcal{R}-\frac{d^{2} \ln \Omega}{d \lambda^{2}}+\left(\frac{d \ln \Omega}{d \lambda}\right)^{2}\right]
$$

Por outro lado, sendo o tensor de Weyl invariante conforme, temos que $\tilde{\mathcal{F}}=$ $\frac{1}{\Omega^{4}} \mathcal{F}$, donde reescrevemos (2.37) como

$$
\frac{d^{2} \tilde{\mathcal{D}}}{d \tilde{\lambda}^{2}}(\tilde{\lambda})+\tilde{T} \tilde{\mathcal{D}}(\tilde{\lambda})=0
$$

onde

$$
\tilde{T}=\frac{1}{\Omega^{4}}\left[\left(\begin{array}{cc}
\mathcal{R}+\operatorname{Re} \mathcal{F} & \operatorname{Im} \mathcal{F} \\
\operatorname{Im} \mathcal{F} & \mathcal{R}-\operatorname{Re} \mathcal{F}
\end{array}\right)+\left(\begin{array}{cc}
\left(\frac{d \ln \Omega}{d \lambda}\right)^{2}-\frac{d^{2} \ln \Omega}{d \lambda^{2}} & 0 \\
0 & \left(\frac{d \ln \Omega}{d \lambda}\right)^{2}-\frac{d^{2} \ln \Omega}{d \lambda^{2}}
\end{array}\right)\right] .
$$

Afirmamos que se $\mathcal{D}(\lambda)$ é solução de (2.37) escrita em termos dos objetos calculados com a métrica conforme, então $\tilde{\mathcal{D}}=\Omega \mathcal{D}$ é solução de (2.60). De fato, usando $\frac{d \tilde{\lambda}}{d \lambda}=\Omega^{2}$, temos que

$$
\frac{d^{2} \tilde{\mathcal{D}}}{d \tilde{\lambda}^{2}}=\frac{1}{\Omega^{3}}\left[\frac{d^{2} \mathcal{D}}{d \lambda^{2}}-\mathcal{D}\left(\frac{d \ln \Omega}{d \lambda}\right)^{2}+\mathcal{D} \frac{d^{2} \ln \Omega}{d \lambda^{2}}\right]
$$

$\operatorname{Mas} \frac{d^{2} \mathcal{D}}{d \lambda^{2}}=-T \mathcal{D}$ e portanto $\frac{d^{2} \tilde{\mathcal{D}}}{d \tilde{\lambda}^{2}}=-\tilde{T} \tilde{\mathcal{D}}$

NotAÇÃo: Deste ponto em diante aboliremos a multidão de tils que trouxemos à cena e estaremos sempre calculando na métrica completa, e não em métricas conformes.

OBSERVAÇÃo: Manteremos a hipótese de que as seções espaciais do espaço de FLRW são planas e portanto escreveremos $\mathcal{D}(\lambda)=\lambda$.

\subsubsection{Aproximação de Born}

Retomando (2.45) e inserindo a forma obtida para $\mathcal{R}$ e $\mathcal{F}$ obtemos a seguinte expressão: 
58 Evolução de feixes de geodésicas nulas e lentes gravitacionais

$$
\left(\frac{\mathcal{D}^{(1)}}{\mathcal{D}^{(0)}}\right)_{a b}=-2 \int_{0}^{\lambda} \frac{\left(\lambda^{\prime}\right)\left(\lambda-\lambda^{\prime}\right)}{(\lambda)} \nabla_{a} \nabla_{b} \Psi_{W} d \lambda^{\prime},
$$

com $a, b=1,2$. O objeto $\Psi_{W}$ é chamado potencial de Weyl e é definido como:

$$
\Psi_{W}=\frac{1}{2}(\Phi+\Psi) .
$$

Até este ponto não sabemos entretanto como são as geodésicas nulas em FLRW perturbado, sobre os pontos das quais, no domínio conveniente do parâmetro afim, o potencial de Weyl tem que ser calculado. Tampouco sabemos exatamente quais as direções em que suas derivadas devem ser calculadas. Sabemos contudo que anuladas as perturbações, as geodésicas nulas são radiais e parametrizadas pelo tempo conforme, o que nos permite utilizar a tecnologia desenvolvida no Apêndice B para determinar em que pontos o potencial de Weyl deve ser avaliado. Caso escrevamos $x^{\mu}(\lambda)=x^{(0) \mu}(\lambda)+x^{(1) \mu}(\lambda)$ podemos esperar que em ordem linear tenhamos $T=T^{(0)}\left(x^{(0) \mu}(\lambda)+x^{(1) \mu}(\lambda)\right)+T^{(1)}\left(x^{(0) \mu}(\lambda)\right)$. Como a matriz $T^{(0)}$ só depende da curvatura seccional da variedade, que é constante, a contribuição em ordem linear da correção da geodésica não tem influência e podemos, por isso, calcular o potencial de Weyl sobre a trajetória dos fótons no espaço não-perturbado, ou seja, devemos aplicar a aproximação de Born.

Aproveitando a aproximação de Born abandonaremos o parâmetro $\lambda$ em favor do tempo conforme $\eta$ e, usando $c=1$, reescrevemos (2.62) como:

$$
\begin{aligned}
\left(\frac{\mathcal{D}^{(1)}}{\mathcal{D}^{(0)}}\right)_{a b}\left[\left(\eta_{o}-\eta\right),\left(\eta_{o}-\eta\right) \mathbf{e}\right] & =-2 \int_{\eta_{o}-\eta}^{\eta_{o}} \frac{\left(\eta_{o}-\eta^{\prime}\right)\left(\eta^{\prime}-\eta\right)}{\left(\eta_{o}-\eta\right)} \\
& \times \nabla_{a} \nabla_{b} \Psi_{W}\left[\left(\eta_{o}-\eta^{\prime}\right),\left(\eta_{o}-\eta^{\prime}\right) \mathbf{e}\right] d \eta^{\prime}
\end{aligned}
$$

com e indicando a direção radial ao longo da qual se realizam as observações e as derivadas são tomadas nas direções ortogonais a e e mutuamente ortogonais entre si.

\subsubsection{Base adequada para tratar lentes na esfera celeste}

Há uma série de aplicações em que se estuda apenas uma pequena região do céu, justificando a aproximação desta região como plana e tomando então a base canônica de vetores de $\mathbb{R}^{2}$ como base ortonormal em $\tilde{T}_{p} M / \operatorname{span}(k)$. Há, no entanto, casos em que isso não é possível e temos que considerar regiões da 
esfera celeste. É importante portanto determinar as coordenadas que devemos empregar neste contexto.

Considerando a trajetória do raio de luz como radial, somos levados a tomar como candidatos naturais para $E_{1}$ e $E_{2}$ os vetores ortogonais sobre a superfície da esfera celeste $\mathbf{e}_{\theta}$ e $\mathbf{e}_{\phi}$. Esses dois vetores, no entanto, não são ortonormais e somos então conduzidos a tomar

$$
E_{1}\left(\eta_{o}-\eta\right)=\frac{1}{\left(\eta_{o}-\eta\right)} \mathbf{e}_{\theta}=: \hat{\mathbf{e}}_{\theta} \quad E_{2}\left(\eta_{o}-\eta\right)=\frac{1}{\left(\eta_{o}-\eta\right) \operatorname{sen} \theta} \mathbf{e}_{\phi}=: \hat{\mathbf{e}}_{\phi} .
$$

Passamos portanto a ter uma base ortonormal de vetores sobre uma esfera. Convém notar que esta base é não-coordenada e portanto o comutador entre elementos da base não é nulo (ver Apêndice D).

Com esta escolha de base [45],

$$
\left(\frac{\mathcal{D}^{(1)}}{\mathcal{D}^{(0)}}\right)_{a b}\left[\left(\eta_{o}-\eta\right),\left(\eta_{o}-\eta\right) \mathbf{e}\right]=-2 \int_{\eta_{o}-\eta}^{\eta_{o}} \frac{\left(\eta^{\prime}-\eta\right)}{\left(\eta_{o}-\eta\right)\left(\eta_{o}-\eta^{\prime}\right)} \nabla_{a} \nabla_{b} \Psi_{W}\left[\left(\eta_{o}-\eta^{\prime}\right),\left(\eta_{o}-\eta^{\prime}\right) \mathbf{e}\right] d \eta^{\prime},
$$

mas sendo agora $a, b=\hat{\mathbf{e}}_{\theta}, \hat{\mathbf{e}}_{\phi}$, sobre a esfera de raio unitário.

\subsubsection{O mapa de lente}

Até este ponto formulamos uma equação diferencial que determina a evolução de componentes de campos de Jacobi em um subespaço de dimensão 2 que nos era particularmente conveniente e fomos capazes de resolvê-la perturbativamente para o caso de um espaço-tempo tipo FLRW sujeito a perturbações escalares. Sabemos que a solução que encontramos corresponde efetivamente à determinação de uma classe de Jacobi, já que a estabelecemos no espaço quociente. O Lema 2.2, entretanto, afirma que para uma dada classe de Jacobi em $\bar{J} \in \tilde{T}_{\gamma(\lambda)} M / \operatorname{span}\left(\gamma^{\prime}(\lambda)\right)$ existe um campo de Jacobi suave em $J \in \tilde{T}_{\gamma(\lambda)} M$ tal que $\bar{J}=[J]$ e portanto existe uma geodésica nula do espaço-tempo que tem este campo de Jacobi como seu campo variacional. É esta geodésica que tomaremos como o percurso efetivo do raio de luz, relativo à geodésica fiducial do feixe que, seguindo a aproximação de Born, deve ser tomada como uma geodésica do espaço-tempo não perturbado.

Seguindo a maneira como formulamos o problema do afastamento de geodésicas em um feixe, sabemos que o afastamento entre duas geodésicas que têm uma velocidade relativa de afastamento $\boldsymbol{\theta}$ é dado por (2.34):

$$
\xi=\mathcal{D}\left(\eta_{o}-\eta\right) \boldsymbol{\theta}
$$


60 Evolução de feixes de geodésicas nulas e lentes gravitacionais

Por podermos escrever $\mathcal{D}=\mathcal{D}^{(0)} \mathbb{1}+\mathcal{D}^{(1)}$ - e lembrando que $\mathcal{D}^{(0)}\left(\eta_{o}-\eta\right)=$ $\left(\eta_{o}-\eta\right)$ - é possível fazer a seguinte interpretação geométrica do fenômeno de lentes gravitacionais fracas em FLRW sujeito a perturbações escalares:

- $a(\eta)\left(\eta_{o}-\eta\right) \boldsymbol{\theta}$ corresponde à separação que teriam a geodésica fiducial e a que tem velocidade de afastamento relativa $\boldsymbol{\theta}$ com relação a ela no espaçotempo de base, ou seja, FLRW sem perturbações;

- $a(\eta) \boldsymbol{\xi}\left(\eta_{o}-\eta\right)$ corresponde à separação efetiva entre geodésica no espaço perturbado e a fiducial;

- $a(\eta)\left(\eta_{o}-\eta\right) \boldsymbol{\theta}-a(\eta) \boldsymbol{\xi}$ é a diferença entre o afastamento que uma geodésica com velocidade relativa de afastamento $\boldsymbol{\theta}$ em relação a fiducial teria no espaço-tempo não perturbado, e a que tem no espaço-tempo sujeito a perturbações. Esta diferença, dada por $-a(\eta) \mathcal{D}^{(1)} \boldsymbol{\theta}$, corresponde então à correção incorporada pela equação de lentes.

Consequentemente [46],

$$
\begin{aligned}
\boldsymbol{\alpha} & :=\frac{a(\eta)\left(\eta_{o}-\eta\right) \boldsymbol{\theta}-a(\eta) \boldsymbol{\xi}\left[\left(\eta_{o}-\eta\right),\left(\eta_{o}-\eta\right) \mathbf{e}\right]}{a(\eta)\left(\eta_{o}-\eta\right)} \\
& =\left(2 \int_{\eta_{o}-\eta}^{\eta_{o}} \frac{\left(\eta_{o}-\eta^{\prime}\right)\left(\eta^{\prime}-\eta\right)}{\left(\eta_{o}-\eta\right)} \nabla_{a} \nabla_{b} \Psi_{W}\left[\left(\eta_{o}-\eta^{\prime}\right),\left(\eta_{o}-\eta^{\prime}\right) \mathbf{e}\right] d \eta^{\prime}\right) \boldsymbol{\theta} \\
& =\nabla_{a} \nabla_{b}\left(2 \int_{\eta_{o}-\eta}^{\eta_{o}} \frac{\left(\eta_{o}-\eta^{\prime}\right)\left(\eta^{\prime}-\eta\right)}{\left(\eta_{o}-\eta\right)} \Psi_{W}\left[\left(\eta_{o}-\eta^{\prime}\right),\left(\eta_{o}-\eta^{\prime}\right) \mathbf{e}\right] d \eta^{\prime}\right) \boldsymbol{\theta} \\
& =\left[\operatorname{Hess}\left(\psi\left[\left(\eta_{o}-\eta^{\prime}\right),\left(\eta_{o}-\eta^{\prime}\right) \mathbf{e}\right]\right)\right] \boldsymbol{\theta}
\end{aligned}
$$

onde $\psi$ é dito o potencial de lente:

$$
\begin{aligned}
\psi\left[\left(\eta_{o}-\eta\right),\left(\eta_{o}-\eta\right) \mathbf{e}\right]:= & 2 \int_{\eta_{o}-\eta}^{\eta_{o}} \frac{\left(\eta_{o}-\eta^{\prime}\right)\left(\eta^{\prime}-\eta\right)}{\left(\eta_{o}-\eta\right)} \\
& \times \Psi_{W}\left[\left(\eta_{o}-\eta^{\prime}\right),\left(\eta_{o}-\eta^{\prime}\right) \mathbf{e}\right] d \eta^{\prime}
\end{aligned}
$$

e Hess $(\psi)$ representa o Hessiano do potencial de lente. Devemos notar que $\boldsymbol{\alpha}$ e $\boldsymbol{\theta}$ são vetores de duas dimensões e o Hessiano é uma matriz $2 \times 2$.

Definindo:

$$
\boldsymbol{\beta}(\boldsymbol{\theta}, \mathbf{e}, \eta):=\frac{\boldsymbol{\xi}\left[\left(\eta_{o}-\eta\right),\left(\eta_{o}-\eta\right) \mathbf{e}\right]}{\left(\eta_{o}-\eta\right)}
$$

podemos escrever o mapa de lente:

$$
\boldsymbol{\beta}(\boldsymbol{\theta}, \mathbf{e})=\boldsymbol{\theta}-\boldsymbol{\alpha}(\boldsymbol{\theta}, \mathbf{e}) .
$$


A dependência em $\eta$ não foi escrita para aliviar a notação.

Notamos que esta expressão é geral para descrever desvio transversal das geodésicas em ordem linear. Procederemos agora a mais algumas aproximações, consoante com o fato de termos expressões que descrevem desvio em ordem linear. Para tanto, vamos escolher um vetor $\mathbf{l}$ tal que $\boldsymbol{\theta}=\mathbf{l}-\mathbf{e}$ (ou esteja em sua classe de equivalência) e, como $\boldsymbol{\theta}$ é pequeno, vamos escrever, como primeiro termo na expansão em série de Taylor de $\nabla \psi\left[\left(\eta_{o}-\eta\right),\left(\eta_{o}-\eta\right) \mathbf{l}\right]$ :

$$
\left.\operatorname{Hess}(\psi)\right|_{\left[\left(\eta_{o}-\eta\right),\left(\eta_{o}-\eta\right) \mathbf{e}\right]} \boldsymbol{\theta} \approx \nabla \psi\left[\left(\eta_{o}-\eta\right),\left(\eta_{o}-\eta\right) \mathbf{l}\right]-\nabla \psi\left[\left(\eta_{o}-\eta\right),\left(\eta_{o}-\eta\right) \mathbf{e}\right]
$$

e, portanto,

$$
\begin{aligned}
\boldsymbol{\alpha}(\boldsymbol{\theta}, \mathbf{e}) & =\nabla \psi\left[\left(\eta_{o}-\eta\right),\left(\eta_{o}-\eta\right) \mathbf{l}\right]-\nabla \psi\left[\left(\eta_{o}-\eta\right),\left(\eta_{o}-\eta\right) \mathbf{e}\right] \\
& =\nabla\left(\psi\left[\left(\eta_{o}-\eta\right),\left(\eta_{o}-\eta\right) \mathbf{l}\right]-\psi\left[\left(\eta_{o}-\eta\right),\left(\eta_{o}-\eta\right) \mathbf{e}\right]\right)
\end{aligned}
$$

Como tomar a diferença entre os potenciais apenas introduz um termo que depende da distância ao observador, renomearemos a diferença entre os potenciais de $\psi\left[\left(\eta_{o}-\eta\right),\left(\eta_{o}-\eta\right) \mathbf{l}\right][47]$. Desta maneira, escrevemos:

$$
\boldsymbol{\alpha}(\boldsymbol{\theta})=\nabla \psi\left[\left(\eta_{o}-\eta\right),\left(\eta_{o}-\eta\right) \mathbf{l}\right] .
$$

Devemos notar que ângulos absolutos não podem ser conhecidos pois todos são determinados em termos de um raio fiducial arbitrariamente escolhido e portanto $\boldsymbol{\alpha}$ é não único. Apenas efeitos que dependam de derivadas deste ângulo de deflexão fazem sentido.

Podemos interpretar (2.68) com o auxílio da Figura 2.2.

Nesta figura o ponto $\mathrm{B}$ deve corresponder à posição real da fonte que $\mathrm{o}$ observador teria atribuído ao ponto $\mathrm{C}$ caso não houvesse desvio. A distância $B C$ é o tamanho da correção incorporada pelo gradiente do potencial de lente.

Devemos notar que quando usarmos a base de vetores definida sobre a esfera, devemos escrever o potencial de lente como [48]:

$$
\begin{aligned}
\psi\left[\left(\eta_{o}-\eta\right),\left(\eta_{o}-\eta\right) \mathbf{e}\right]:= & 2 \int_{\eta_{o}-\eta}^{\eta_{o}} \frac{\left(\eta_{o}-\eta\right)}{\left(\eta_{o}-\eta^{\prime}\right)\left(\eta^{\prime}-\eta\right)} \\
& \times \Psi_{W}\left[\left(\eta_{o}-\eta^{\prime}\right),\left(\eta_{o}-\eta^{\prime}\right) \mathbf{e}\right] d \eta^{\prime} .
\end{aligned}
$$


62 Evolução de feixes de geodésicas nulas e lentes gravitacionais

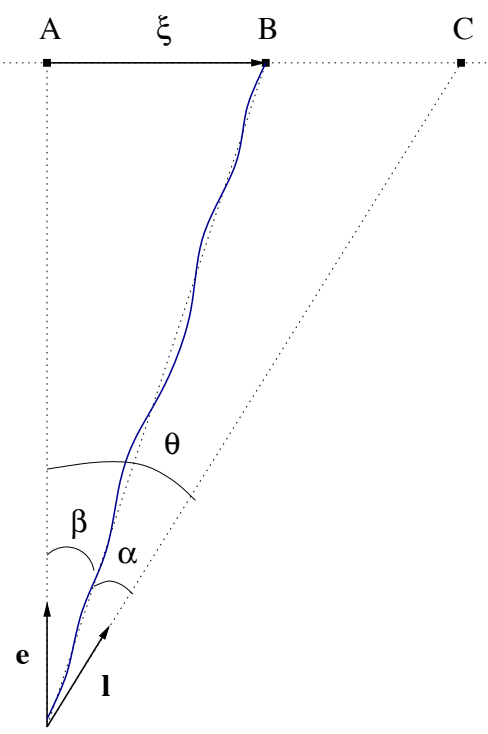

Fig. 2.2 Elementos presentes no mapa de lente. $\boldsymbol{\theta}$ é a direção a que se associaria a imagem de uma fonte localizada no ponto B caso não se tomasse em conta o efeito das lentes gravitacionais. $\alpha$ é o ângulo de correção. 


\section{3}

\section{Equações de Boltzmann para temperatura e polarização da CMB no espaço das posições}

Antes de dirigirmo-nos ao problema das lentes gravitacionais sobre a CMB, dedicaremo-nos a desenvolver um formalismo para tratar a temperatura e polarização da CMB no espaço das posições. Os resultados aqui apresentados serão reunidos a elementos do Capítulo 2 e aplicados no Capítulo 4 ao problema das lentes gravitacionais sobre a radiação cósmica de fundo.

Tomaremos aqui a versão integral da hierarquia de Boltzmann para a descrição da polarização e temperatura da $\mathrm{CMB}$ e analisaremos a possibilidade de reescrever-se o sistema no espaço das posições (ao invés de escrevê-lo no espaço dos momentos), como iniciado em [4]. Mostraremos que a temperatura e a polarização da CMB podem ser descritas no espaço das posições e que densidades de probabilidade associadas à posição de caminhantes executando vôos aleatórios são centrais nessa descrição.

Buscaremos interpretar o sistema físico em termos de propriedades diretamente derivadas da estrutura das equações, sem recorrer a detalhes da origem microscópica de cada termo que nelas compareça. Dito de outra forma, buscaremos compreender um sistema descrito em termos de um conjunto de equações integrais acopladas mantendo-nos afastados, tanto quanto possível, de qualquer ingrediente não diretamente derivado das equações. Sobre a natureza física do problema, manteremos em mente apenas que o sistema de equações de que partimos pretende descrever temperatura e polarização de feixes de fótons inicialmente em equilíbrio com matéria e que tiveram seus estados de polarização alterados por espalhamentos Thomson durante uma fase semi-acoplada entre fótons e elétrons da história térmica do universo conhecida como recombinação. Para ver derivações das equações de Boltzmann de que partimos - e outros ingredientes típicos nas discussões do assunto mas sobre os quais não venhamos a 
64 Equações de Boltzmann para temperatura e polarização da CMB no espaço das posições ater-nos e que eventualmente surjam durante os desenvolvimentos que seguem - indicamos obras de referência da literatura como [49], [50], [51] ou ainda (não constituindo obra de referência da literatura) [52].

Devemos notar, antes de avançar, que as conexões mostradas na seqüência são possíveis por causa de uma série de resultados matemáticos demonstrados no Apêndice C. Decidimos discutir os resultados matemáticos em um apêndice para manter o texto do capítulo mais focado o possível nos aspectos físicos do problema.

Os resultados deste capítulo foram publicados em [3].

\subsection{As equações de Boltzmann}

As equações de Boltzmann para a evolução da temperatura e polarização da CMB escrevem-se [52]:

TEMPERATURA

Escreve-se o contraste de temperatura como:

$$
\Theta\left(\mathbf{x}_{o}, \eta_{o}, \mathbf{o}\right)=\int \frac{d^{3} \mathbf{k}}{(2 \pi)^{3 / 2}} 4 \pi \sum_{l m} i^{l} \Theta_{l}\left(\mathbf{k}, \eta_{o}\right) \mathrm{e}^{i \mathbf{k} \cdot \mathbf{x}_{o}} \mathrm{Y}_{l m}^{*}(\hat{\mathbf{k}}) \mathrm{Y}_{l m}(\mathbf{o})
$$

com

$$
\begin{aligned}
\Theta_{l}\left(\mathbf{k}, \eta_{o}\right)= & \int_{0}^{\eta_{o}} \mathrm{e}^{-\mu(\eta)}\left\{\mu ^ { \prime } ( \eta ) \left[\Theta_{S W}(\mathbf{k}, \eta) j_{l}\left(k\left(\eta_{o}-\eta\right)\right)-V_{b}(\mathbf{k}, \eta) \frac{\partial j_{l}\left(k\left(\eta_{o}-\eta\right)\right)}{\partial \eta}\right.\right. \\
& \left.+\frac{1}{2}\left[\Theta_{2}(\mathbf{k}, \eta)-\sqrt{6} \alpha_{2}^{(2)}(\mathbf{k}, \eta)\right]\left[\frac{1}{2}\left(3 j_{l}^{\prime \prime}\left(k\left(\eta_{o}-\eta\right)\right)+j_{l}\left(k\left(\eta_{o}-\eta\right)\right)\right)\right]\right] \\
& \left.+\left(\Psi^{\prime}+\Phi^{\prime}\right)(\mathbf{k}, \eta) j_{l}\left(k\left(\eta_{o}-\eta\right)\right)\right\} d \eta .
\end{aligned}
$$

POLARIZAÇÃO

Escreve-se, em termos dos parâmetros de Stokes $Q, U$ e $I$ a polarização linear:

$$
\frac{Q+i U}{4 I}\left(\mathbf{x}_{o}, \eta_{o}, \mathbf{o}\right)=\int \frac{d^{3} \mathbf{k}}{(2 \pi)^{3 / 2}} 4 \pi \sum_{l m} i^{l} \alpha_{l}^{(2)}\left(\mathbf{k}, \eta_{o}\right) \mathrm{e}^{i \mathbf{k} \cdot \mathbf{x}_{o}} \mathrm{Y}_{l m}^{*}(\hat{\mathbf{k}})_{2} \mathrm{Y}_{l m}(\mathbf{o})
$$


com

$$
\begin{aligned}
\alpha_{l}^{(2)}\left(\mathbf{k}, \eta_{o}\right)= & -\frac{3}{2} \sqrt{\frac{(l+2) !}{(l-2) !}} \int_{0}^{\eta_{o}} d \eta \mu^{\prime}(\eta) \mathrm{e}^{-\mu(\eta)} \\
& \times \frac{1}{2}\left[\Theta_{2}(\mathbf{k}, \eta)-\sqrt{6} \alpha_{2}^{(2)}(\mathbf{k}, \eta)\right] \frac{j_{l}\left(k\left(\eta_{o}-\eta\right)\right)}{\left(k\left(\eta_{o}-\eta\right)\right)^{2}}
\end{aligned}
$$

Em (3.3) a expansão é feita em termos de harmônicos esféricos de spin. Descrevemos a natureza desses objetos no Apêndice D.

Costuma-se denotar $P(\mathbf{k}, \eta)=\frac{1}{2}\left[\Theta_{2}(\mathbf{k}, \eta)-\sqrt{6} \alpha_{2}^{(2)}(\mathbf{k}, \eta)\right]$ o termo de fonte para a polarização.

Nestas expressões $\Theta_{S W}, V_{b}, \Phi$ e $\Psi$ são, respectivamente, os termos de SachsWolfe, a velocidade dos bárions e os potenciais escalares de perturbação cosmológica, que são todos os elementos invariantes de gauge que parametrizam perturbações escalares lineares em FLRW [50]. $\mathrm{e}^{-\mu(\eta)} \mu^{\prime}(\eta)$ é a função de visibilidade [51], $\eta$ é o tempo conforme. Intervalos do tipo $\left(\eta_{o}-\eta\right)$ correspondem diretamente a intervalos (conformes) espaciais, pois tomamos $c=1$. Subindices o são relativos ao observador. Linhas indicam, como usual, derivação com relação ao tempo conforme.

Apenas para que se tenha idéia da forma da função de visibilidade apresentamos uma figura construída a partir de um modelo simplificado de recombinação apresentado em [51]:

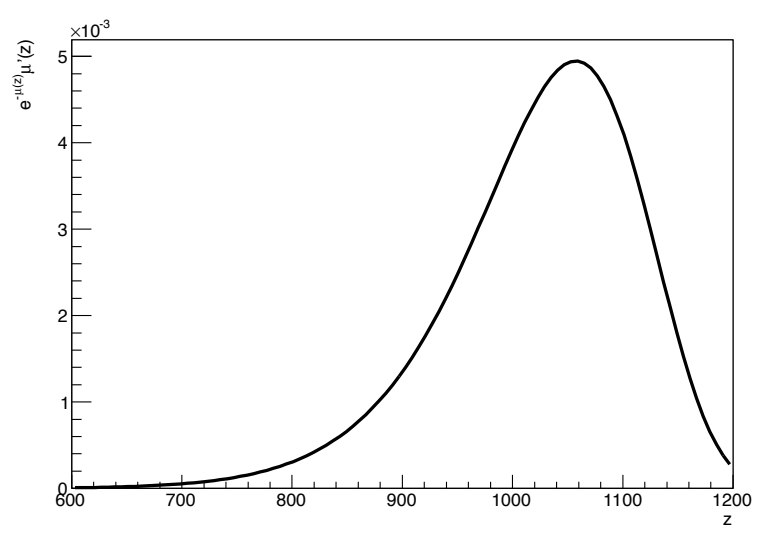

Fig. 3.1 Forma da função de visibilidade para um modelo simplificado de recombinação apresentado em [51]. Espalhamentos são frequentes em torno de $z=1100$ e há aumento do livre caminho médio dos fotos para zs menores. 
66 Equações de Boltzmann para temperatura e polarização da CMB no espaço das posições

Essa função descreve a probabilidade que um fóton se espalhe com um elétron em um instante $\eta$ dado, e que não se espalhe novamente até sua observação.

Juntamente com as equações de Einstein que determinam a evolução dos potenciais cosmológicos, (3.1) e (3.3) formam um sistema de equações equivalentes à hierarquia de equações de Boltzmann.

\subsection{Estudo do sistema quando se toma $P=0$ na temperatura}

Vamos inicialmente desligar os efeitos da radiação polarizada entre os termos de fonte para a flutuação de temperatura, fazendo $P=0$ na equação para a temperatura. Especificamente, analisaremos o sistema anteriormente apresentado, ou seja, (3.1), (3.2), (3.3), (3.4) com (3.2) substituída por:

$$
\begin{aligned}
\Theta_{l}\left(\mathbf{k}, \eta_{o}\right)= & \int_{0}^{\eta_{o}} \mathrm{e}^{-\mu(\eta)}\left\{\mu^{\prime}(\eta)\left[\Theta_{S W}(\mathbf{k}, \eta) j_{l}\left(k\left(\eta_{o}-\eta\right)\right)-V_{b}(\mathbf{k}, \eta) \frac{\partial j_{l}\left(k\left(\eta_{o}-\eta\right)\right)}{\partial \eta}\right]\right. \\
& \left.+\left(\Psi^{\prime}+\Phi^{\prime}\right)(\mathbf{k}, \eta) j_{l}\left(k\left(\eta_{o}-\eta\right)\right)\right\} d \eta
\end{aligned}
$$

Com efeito, os termos desprezados nesta aproximação são de ordem superior se o processo iterativo de solução das equações integrais faz sentido. A eliminação dos termos de fonte associados à radiação polarizada na equação para a temperatura não é essencial, mas simplifica bastante o tratamento. Posteriormente reintroduziremos o efeito desses termos. Como apresenta-se, (3.5) não é mais que a expressão dos efeitos Sachs-Wolfe e Sachs-Wolfe integrado, escritos como uma integral sobre a linha de visão. A expressão descreve a maneira pela qual a temperatura dos fótons é influenciada pelo meio de onde emergiram e pelas inhomogeneidades características que atravessaram durante sua propagação até serem observados.

obSERVAÇÃO: TOMAREMOS $\mathbf{x}_{o}=\mathbf{0}$ No QUE SEgUe, OU SEJA, O OBSERVADOR É ESCOLHIDO COMO A ORIGEM DO SISTEMA DE COORDENADAS. 


\subsubsection{Expressão da temperatura no espaço das posições}

Estudaremos (3.1) mas escreveremos, por conveniência, a mesma decomposição da seguinte forma:

$$
\Theta\left(\eta_{o}, \mathbf{o}\right)=\sum_{l m} \theta_{l m}^{(0)}\left(\eta_{o}\right) \mathrm{Y}_{l m}(\mathbf{o})
$$

e desejamos determinar os coeficientes $\theta_{l m}^{(0)}\left(\eta_{o}\right)$.

Com efeito,

$$
\begin{aligned}
\theta_{l m}^{(0)}\left(\eta_{o}\right)= & 2 i^{l} \int_{0}^{\eta_{o}} d \eta \int \frac{d k}{(2 \pi)^{1 / 2}} k^{2}\left\{\int d ^ { 2 } \hat { \mathbf { k } } \mathrm { e } ^ { - \mu ( \eta ) } \left\{\mu ^ { \prime } ( \eta ) \left[\Theta_{S W}(\mathbf{k}, \eta)\right.\right.\right. \\
& \left.\left.\left.-V_{b}(\mathbf{k}, \eta) \frac{\partial}{\partial \eta}\right]+\left(\Psi^{\prime}+\Phi^{\prime}\right)(\mathbf{k}, \eta)\right\} \mathrm{Y}_{l m}^{*}(\hat{\mathbf{k}})\right\} j_{l}\left(k\left(\eta_{o}-\eta\right)\right)
\end{aligned}
$$

Definiremos então o seguinte objeto:

$S_{l m}(k, \eta):=\int d^{2} \hat{\mathbf{k}} \mathrm{e}^{-\mu(\eta)}\left\{\mu^{\prime}(\eta)\left[\Theta_{S W}(\mathbf{k}, \eta)-V_{b}(\mathbf{k}, \eta) \frac{\partial}{\partial \eta}\right]+\left(\Psi^{\prime}+\Phi^{\prime}\right)(\mathbf{k}, \eta)\right\} \mathrm{Y}_{l m}^{*}(\hat{\mathbf{k}})$

e com isso reescrevemos (3.7) como:

$$
\theta_{l m}^{(0)}\left(\eta_{o}\right)=2 i^{l} \int_{0}^{\eta_{o}} d \eta \int \frac{d k}{(2 \pi)^{1 / 2}} k^{2} S_{l m}(k, \eta) j_{l}\left(k\left(\eta_{o}-\eta\right)\right) .
$$

Observamos agora que os coeficientes $S_{l m}(k, \eta)$ no espaço dos momentos podem ser expressos no espaço das posições ${ }^{1}$ Como resultado, teremos:

$$
\theta_{l m}^{(0)}\left(\eta_{o}\right)=\frac{2}{\pi} \int_{0}^{\eta_{o}} d \eta \int d x x^{2} S_{l m}(x, \eta) \int d k k^{2} j_{l}(k x) j_{l}\left(k\left(\eta_{o}-\eta\right)\right) .
$$

Como

$$
\int_{0}^{\infty} d k k^{2} j_{L}(a k) j_{L}(b k)=\frac{\pi}{2} \frac{b^{L}}{a^{L+2}} \delta(a-b),
$$

obtemos finalmente o resultado simples:

${ }^{1}$ Seja $y_{l m}(k, A)$ os coeficientes da expansão em harmônicos esféricos de uma quantidade $y$ que depende de $k$ e mais um grupo de variáveis denotado por $A$. Esses coeficientes no espaço de Fourier são relacionados aos correspondentes coeficientes no espaço das posições como:

$$
y_{l m}(k, A)=2(-i)^{l} \int_{0}^{\infty} y_{l m}(x, A) j_{l}(k x) x^{2} \frac{d x}{(2 \pi)^{1 / 2}} .
$$


68 Equações de Boltzmann para temperatura e polarização da CMB no espaço das posições

$$
\theta_{l m}^{(0)}\left(\eta_{o}\right)=\int_{0}^{\eta_{o}} S_{l m}\left(\left(\eta_{o}-\eta\right), \eta\right) d \eta
$$

$\mathrm{Ou}$ seja, apenas termos de fonte sobre uma esfera de raio $\left(\eta_{o}-\eta\right)$ contribuem para o valor de $\theta_{l m}^{(0)}\left(\eta_{o}\right)$ para cada $\eta$.

\subsubsection{Expressão da polarização no espaço das posições}

Desejamos agora reescrever (3.3) como:

$$
\frac{Q+i U}{4 I}\left(\eta_{o}, \mathbf{o}\right)=\sum_{l m} \pi_{l m}\left(\eta_{o}\right)_{2} \mathrm{Y}_{l m}(\mathbf{o})
$$

e determinar os coeficientes $\pi_{l m}\left(\eta_{o}\right)$.

Observemos um momento a expressão (3.4): os termos de fonte são $\Theta_{2}$ e $\alpha_{2}^{(2)}$. Considerando um processo iterativo de solução, o primeiro passo deve ter $\alpha_{2}^{(2)}=0$ e apenas $\Theta_{2}$ como fonte. Seguindo o processo iterativo teremos $\pi_{l m}=\pi_{l m}^{(1)}+\pi_{l m}^{(2)}+\ldots$ onde cada $\pi_{l m}^{(j)}$ representa um nível $j$ de inclusão das fontes. O cálculo dos coeficientes $\pi_{l m}$ é feito incorporando termos até um determinado grau de precisão desejado, assumindo convergência (ou pelo menos convergência assintótica) da série. O termo de ordem zero da expansão é nulo. Calculemos a contribuição da primeira iteração (apenas $\Theta_{2}$ como fonte de polarização):

$$
\begin{aligned}
\pi_{l m}^{(1)}\left(\eta_{o}\right)= & -\frac{3}{4} 2 i^{l} \sqrt{\frac{(l+2) !}{(l-2) !}} \int_{0}^{\eta_{o}} d \eta_{s} \int_{0}^{\eta_{s}} d \eta \int \frac{d k}{(2 \pi)^{1 / 2}} k^{2} S_{l m}(k, \eta) \\
& \times j_{2}\left(k\left(\eta_{s}-\eta\right)\right) \frac{j_{l}\left(k\left(\eta_{o}-\eta_{s}\right)\right)}{\left(k\left(\eta_{o}-\eta_{s}\right)\right)^{2}} \mu^{\prime}\left(\eta_{s}\right) \mathrm{e}^{-\mu\left(\eta_{s}\right)}
\end{aligned}
$$

para os mesmos $S_{l m}$ definidos em (3.8). Novamente relacionamos $S_{l m}(k, \eta)$ e $S_{l m}(x, \eta)$, e obtemos:

$$
\begin{aligned}
\pi_{l m}^{(1)}\left(\eta_{o}\right)= & -\frac{3}{2 \pi} \sqrt{\frac{(l+2) !}{(l-2) !}} \int_{0}^{\eta_{o}} d \eta_{s} \mu^{\prime}\left(\eta_{s}\right) \mathrm{e}^{-\mu\left(\eta_{s}\right)} \int_{0}^{\eta_{s}} d \eta \int_{0}^{\infty} d x x^{2} S_{l m}(x, \eta) \\
& \times \int_{0}^{\infty} d k k^{2} j_{l}(k x) \frac{j_{l}\left(k\left(\eta_{o}-\eta_{s}\right)\right)}{\left(k\left(\eta_{o}-\eta_{s}\right)\right)^{2}} j_{2}\left(k\left(\eta_{s}-\eta\right)\right)
\end{aligned}
$$

Indo um passo adiante, podemos tomar em conta o efeito de $\pi_{l m}^{(1)}$ como fonte para a polarização, ou seja, incorporar o primeiro nível de $\alpha_{2}^{(2)}$ como fonte. Tomar em conta esta contribuição corresponde a calcular o termo $\pi_{l m}^{(2)}$ na expansão de $\pi_{l m}$ : 


$$
\begin{aligned}
\pi_{l m}^{(2)}\left(\eta_{o}\right)= & -\frac{3}{2} \sqrt{\frac{(l+2) !}{(l-2) !}} \int_{0}^{\eta_{o}} d \eta_{s} \mu^{\prime}\left(\eta_{s}\right) \mathrm{e}^{-\mu\left(\eta_{s}\right)} \int \frac{d^{3} \mathbf{k}}{(2 \pi)^{3 / 2}} 4 \pi i^{l}\left[-\frac{\sqrt{6}}{2} \alpha_{2}^{(2)}\left(\mathbf{k}, \eta_{s}\right)\right] \\
& \times \mathrm{Y}_{l m}^{*}(\hat{\mathbf{k}}) \frac{j_{l}\left(k\left(\eta_{o}-\eta_{s}\right)\right)}{\left(k\left(\eta_{o}-\eta_{s}\right)\right)^{2}} \\
= & -\frac{3}{2} \sqrt{\frac{(l+2) !}{(l-2) !}} \int_{0}^{\eta_{o}} d \eta_{s} \mu^{\prime}\left(\eta_{s}\right) \mathrm{e}^{-\mu\left(\eta_{s}\right)} 4 \pi i^{l} \frac{1}{2 \pi}\left[\frac { - \sqrt { 6 } } { 2 } \left(-\frac{3}{2} \sqrt{24} \int_{0}^{\eta_{s}} d \eta_{i} \mu^{\prime}\left(\eta_{i}\right) \mathrm{e}^{-\mu\left(\eta_{i}\right)}\right.\right. \\
& \left.\left.\times \frac{1}{2} \int \frac{d k}{(2 \pi)^{1 / 2}} k^{2} \int d^{2} \mathbf{k} \Theta_{2}\left(\mathbf{k}, \eta_{i}\right) \mathrm{Y}_{l m}^{*}(\hat{\mathbf{k}}) \frac{j_{2}\left(k\left(\eta_{s}-\eta_{i}\right)\right)}{\left(k\left(\eta_{s}-\eta_{i}\right)\right)^{2}}\right)\right] \frac{j_{l}\left(k\left(\eta_{o}-\eta_{s}\right)\right)}{\left(k\left(\eta_{o}-\eta_{s}\right)\right)^{2}} \\
= & -\frac{3}{2} \sqrt{\frac{(l+2) !}{(l-2) !}} \int_{0}^{\eta_{o}} d \eta_{s} \mu^{\prime}\left(\eta_{s}\right) \mathrm{e}^{-\mu\left(\eta_{s}\right)}\left[9 i^{l}\left(2(-i)^{l}\right) \int_{0}^{\eta_{s}} d \eta_{i} \mu^{\prime}\left(\eta_{i}\right) \mathrm{e}^{-\mu\left(\eta_{i}\right)}\right. \\
& \times \int_{0}^{\eta_{i}} d \eta \int \frac{d k}{(2 \pi)^{1 / 2}} k^{2} \int_{0}^{\infty} \frac{d x}{(2 \pi)^{1 / 2}} x^{2} S_{l m}(x, \eta) j_{2}\left(k\left(\eta_{i}-\eta\right)\right) j_{l}(k x) \\
& \left.\times \frac{j_{2}\left(k\left(\eta_{s}-\eta_{i}\right)\right)}{\left(k\left(\eta_{s}-\eta_{i}\right)\right)^{2}}\right] \frac{j_{l}\left(k\left(\eta_{o}-\eta_{s}\right)\right)}{\left(k\left(\eta_{o}-\eta_{s}\right)\right)^{2}},
\end{aligned}
$$

ou seja,

$$
\begin{aligned}
\pi_{l m}^{(2)}\left(\eta_{o}\right) & =-\frac{3}{2 \pi} \sqrt{\frac{(l+2) !}{(l-2) !}} \int_{0}^{\eta_{o}} d \eta_{s} \mu^{\prime}\left(\eta_{s}\right) \mathrm{e}^{-\mu\left(\eta_{s}\right)} \int_{0}^{\eta_{s}} d \eta_{i} \mu^{\prime}\left(\eta_{i}\right) \mathrm{e}^{-\mu\left(\eta_{i}\right)} \int_{0}^{\eta_{i}} d \eta \int_{0}^{\infty} d x x^{2} \\
& \times S_{l m}(x, \eta)\left[9 \int d k k^{2} j_{l}(k x) \frac{j_{l}\left(k\left(\eta_{o}-\eta_{s}\right)\right)}{\left(k\left(\eta_{o}-\eta_{s}\right)\right)^{2}} \frac{j_{2}\left(k\left(\eta_{s}-\eta_{i}\right)\right)}{\left(k\left(\eta_{s}-\eta_{i}\right)\right)^{2}} j_{2}\left(k\left(\eta_{i}-\eta\right)\right)\right] .(3.15)
\end{aligned}
$$

Exatamente pela mesma sequência de passos mostra-se que:

$$
\begin{aligned}
\pi_{l m}^{(3)}\left(\eta_{o}\right)= & -\frac{3}{2 \pi} \sqrt{\frac{(l+2) !}{(l-2) !}} \int_{0}^{\eta_{o}} d \eta_{f} \mu^{\prime}\left(\eta_{f}\right) \mathrm{e}^{-\mu\left(\eta_{f}\right)} \int_{0}^{\eta_{f}} d \eta_{s} \mu^{\prime}\left(\eta_{s}\right) \mathrm{e}^{-\mu\left(\eta_{s}\right)} \int_{0}^{\eta_{s}} d \eta_{i} \mu^{\prime}\left(\eta_{i}\right) \mathrm{e}^{-\mu\left(\eta_{i}\right)} \\
& \times \int_{0}^{\eta_{i}} d \eta \int_{0}^{\infty} d x x^{2} S_{l m}(x, \eta)\left[9^{2} \int d k k^{2} j_{l}(k x) \frac{j_{l}\left(k\left(\eta_{o}-\eta_{f}\right)\right)}{\left(k\left(\eta_{o}-\eta_{f}\right)\right)^{2}}\right. \\
& \left.\times \frac{j_{2}\left(k\left(\eta_{f}-\eta_{s}\right)\right)}{\left(k\left(\eta_{f}-\eta_{s}\right)\right)^{2}} \frac{j_{2}\left(k\left(\eta_{s}-\eta_{i}\right)\right)}{\left(k\left(\eta_{s}-\eta_{i}\right)\right)^{2}} j_{2}\left(k\left(\eta_{i}-\eta\right)\right)\right]
\end{aligned}
$$

Em geral, 
70 Equações de Boltzmann para temperatura e polarização da CMB no espaço das posições

$$
\begin{aligned}
\pi_{l m}^{(n)}\left(\eta_{o}\right)= & -\frac{3}{2 \pi} \sqrt{\frac{(l+2) !}{(l-2) !}} \int_{0}^{\eta_{o}} d \eta_{f} \mu^{\prime}\left(\eta_{f}\right) \mathrm{e}^{-\mu\left(\eta_{f}\right)} \\
& \times \frac{1}{(n-1) !} \int_{0}^{\eta_{f}} d \eta^{\prime} \ldots d \eta^{\prime \prime} d \eta_{i} \mathrm{~T}\left\{\mu^{\prime}\left(\eta^{\prime}\right) \mathrm{e}^{-\mu\left(\eta^{\prime}\right)} \ldots \mu^{\prime}\left(\eta^{\prime \prime}\right) \mathrm{e}^{-\mu\left(\eta^{\prime \prime}\right)} \mu^{\prime}\left(\eta_{i}\right) \mathrm{e}^{-\mu\left(\eta_{i}\right)}\right\} \\
& \times \int_{0}^{\eta_{i}} d \eta \int_{0}^{\infty} d x x^{2} S_{l m}(x, \eta)\left[9^{(n-1)} \int d k k^{2} j_{l}(k x) \frac{j\left(k\left(\eta_{o}-\eta_{f}\right)\right)}{\left(k\left(\eta_{o}-\eta_{f}\right)\right)^{2}}\right. \\
& \times \underbrace{\frac{j_{2}\left(k\left(\eta_{f}-\eta^{\prime}\right)\right)}{\left(k\left(\eta_{f}-\eta^{\prime}\right)\right)^{2}} \ldots \frac{j_{2}\left(k\left(\eta^{\prime \prime}-\eta_{i}\right)\right)}{\left(k\left(\eta^{\prime \prime}-\eta_{i}\right)\right)^{2}}}_{(n-1) \text { vezes }} j_{2}\left(k\left(\eta_{i}-\eta\right)\right)]
\end{aligned}
$$

onde o intervalo $\left(\eta_{f}-\eta_{i}\right)$ deve ser dividido em $n-1$ subintervalos que serão os argumentos dos termos $\frac{j_{2}(k(.))}{(k(.))^{2}}$. T denota o produto de tempo ordenado destes subintervalos. A ordenação temporal é introduzida para tomar-se em conta os limites superiores de integração nas variáveis temporais, como aparecem em (3.16) por exemplo.

Como conclusão, vemos que todos os termos contribuindo para os coeficientes da expansão da polarização contém integrais do tipo:

$$
\int d k k^{2} j_{l}(k x) \frac{j_{l}\left(k\left(\eta_{o}-\eta_{f}\right)\right)}{\left(k\left(\eta_{o}-\eta_{f}\right)\right)^{2}} \frac{j_{2}\left(k\left(\eta_{f}-\eta^{\prime}\right)\right)}{\left(k\left(\eta_{f}-\eta^{\prime}\right)\right)^{2}} \ldots \frac{j_{2}\left(k\left(\eta^{\prime \prime}-\eta_{i}\right)\right)}{\left(k\left(\eta^{\prime \prime}-\eta_{i}\right)\right)^{2}} j_{2}\left(k\left(\eta_{i}-\eta\right)\right) .
$$

\subsection{Conexão entre a expressão da polarização e processos de vôos aleatórios}

No Apêndice $\mathrm{C}$ damos um tratamento relativamente amplo de integrais envolvendo produtos de funções de Bessel esféricas como as que surgiram em nosso tratamento da polarização no espaço das posições. Demonstramos neste apêndice o seguinte teorema (Teorema C.7):

Vale a igualdade

$$
\begin{aligned}
& \int_{0}^{\infty} d k k^{2} j_{l}(k X) \frac{j_{l}(k R)}{(k R)^{m}} \frac{j_{m}\left(k r_{1}\right)}{\left(k r_{1}\right)^{m}} \frac{j_{m}\left(k r_{2}\right)}{\left(k r_{2}\right)^{m}} \ldots \frac{j_{m}\left(k r_{n-1}\right)}{\left(k r_{n-1}\right)^{m}} j_{m}\left(k r_{n}\right)= \\
& \frac{(-1)^{m}}{2} \frac{\left(\frac{\pi}{2}\right)^{(n+1) / 2}}{(\Gamma(m+3 / 2))^{n-1}} \int d(\cos \alpha) \frac{1}{r^{2}}\left(\frac{X r_{n}}{r^{2}}\right)^{m} P_{l}^{-m}(\cos \alpha) \operatorname{sen}^{m} \alpha \\
& \times p_{n}\left(r ; r_{1}, \ldots, r_{n} \mid 2 m+3\right)
\end{aligned}
$$


Conexão entre a expressão da polarização e processos de vôos aleatórios 71 com $r^{2}=R^{2}+X^{2}-2 R X \cos \alpha, P_{l}^{-m}(\cos \alpha)$ um polinômio de Legendre associado e $p_{n}\left(r ; r_{1}, \ldots, r_{n} \mid 2 m+3\right)$ é a densidade de probabilidade de que após $n$ passos de comprimentos $r_{1}, r_{2}, \ldots, r_{n}$ de um vôo aleatório em $2 m+3$ dimensões, o caminhante esteja a uma distância $r$ da origem do movimento.

Um vôo aleatório é o movimento executado por um caminhante que, sempre com velocidade constante, parte de uma origem e muda de direção em instantes aleatórios. O número de passos do vôo é o número de trechos percorridos e o comprimento de cada trecho é diretamente associado ao tempo transcorrido entre duas mudanças de direção sucessivas, já que a velocidade é sempre constante. Pergunta-se, no estudo deste problema, a distribuição de probabilidades de que após $n$ passos de comprimentos $r_{1}, r_{2}, \ldots, r_{n}$, o caminhante esteja a uma distância $r$ da origem do movimento. Este problema foi introduzido no início do século XX no contexto do estudo de migração de pássaros e cedo recebeu o primeiro tratamento formal quando Lord Rayleigh aplicou o mesmo conceito ao estudo de problemas de acústica. Tratamentos do problema são apresentados, por exemplo, em [53, 54]. A referência [55] apresenta uma revisão histórica do problema. A primeira discussão de vôos aleatórios na literatura relacionada a astrofísica é provavelmente [56].

O aparecimento de densidades de probabilidade associadas a vôos aleatórios no contexto do problema de cosmologia que estamos tratando não poderia ter sido antecipado antes de tratarmos as integrais do tipo (3.18). Esta relação, uma vez demonstrada, entretanto, mostra-se não mais surpreendente: desvios das distribuições de equilíbrio para a temperatura e polarização dos fótons são ocasionadas por espalhamentos Thomson entre fótons e elétrons durante a recombinação ${ }^{2}$. Quando espalhamentos acontecem há mudança de direção do fóton, que sempre tem velocidade constante, ou seja, pode-se descrever a história dos fótons durante a recombinação como um vôo aleatório.

\footnotetext{
${ }^{2}$ Durante a fase da história térmica do universo conhecida como recombinação os fótons têm uma distribuição de energia muito aproximadamente planckiana, com energia típica da ordem de eV e estão sofrendo espalhamentos cada vez menos frequentes com elétrons livres (que estão sendo progressivamente capturados para formar átomos de hidrogênio). Por ser uma fase fora do equilíbrio termodinâmico os espalhamentos sofridos pelos fótons durante a recombinação induzem perturbações no espectro planckiano e também polarização líquida nos feixes de fótons.
} 
72 Equações de Boltzmann para temperatura e polarização da CMB no espaço das posições

Equações do tipo (3.18) podem, então, ser expressas como ${ }^{3}$ :

$$
\begin{aligned}
& \int_{0}^{\infty} d k k^{2} j_{l}(k x) \frac{j_{l}\left(k \Delta \eta_{0}\right)}{\left(k \Delta \eta_{0}\right)^{2}} \frac{j_{2}\left(k \Delta \eta_{1}\right)}{\left(k \Delta \eta_{1}\right)^{2}} \frac{j_{2}\left(k \Delta \eta_{2}\right)}{\left(k \Delta \eta_{2}\right)^{2}} \ldots \frac{j_{2}\left(k \Delta \eta_{n-1}\right)}{\left(k \Delta \eta_{n-1}\right)^{2}} j_{2}\left(k \Delta \eta_{n}\right)= \\
& \frac{1}{2} \frac{\left(\frac{\pi}{2}\right)^{(n+1) / 2}}{(\Gamma(7 / 2))^{n-1}} \int d(\cos \alpha)\left(\frac{x \Delta \eta_{n}}{r^{3}}\right)^{2} P_{l}^{-2}(\cos \alpha) \operatorname{sen}^{2} \alpha \\
& \times p_{n}\left(r ; \Delta \eta_{1}, \ldots, \Delta \eta_{n} \mid 7\right)
\end{aligned}
$$

onde $r^{2}=\left(\Delta \eta_{0}\right)^{2}+x^{2}-2 x\left(\Delta \eta_{0}\right) \cos \alpha$. A notação de $\Delta \eta$ 's pretende significar: $\Delta \eta_{0}=\left(\eta_{o}-\eta_{f}\right), \Delta \eta_{n}=\left(\eta_{i}-\eta\right)$ e os outros $n-1$ intervalos $\Delta \eta$ 's introduzidos correspondem à subdivisão em $n-1$ partes do intervalo $\eta_{f}-\eta_{i}$. Está diretamente ligado ao fato de que apenas o quadrupolo de flutuações da temperatura serve como fonte para geração de polarização da CMB, que a dimensão em que se processam os vôos aleatórios no contexto desse problema seja 7. Uma possível explicação para esta dimensão é que, após separar a dependência angular da CMB de sua dependência radial e temporal, o cone de luz tem apenas dois graus de liberdade restantes. Como um multipolo de ordem $L$ tem $2 L+1$ graus de liberdade, restam-nos $2 L+3$ graus de liberdade efetivo e por serem sempre quadrupolos a contribuir através dos termos de fonte, obtemos as 7 dimensões referidas.

Que surjam distribuições de probabilidade de vôos aleatórios no tratamento da polarização dos fótons da CMB - e também da temperatura como veremos - é positivo para que se possa formular um tratamento da hierarquia de Boltzmann em termos do número de espalhamentos sofridos pelos fótons durante a recombinação. De fato, a presença de $p_{n}\left(r ; \Delta \eta_{1}, \ldots, \Delta \eta_{n} \mid 7\right)$ no termo $\pi_{l m}^{(n)}$ indica que este termo computa a contribuição de fótons que sofreram $n$ espalhamentos durante a recombinação, mostrando efetivamente que a descrição em termos do número de iterações faz sentido.

Continuando o exame de $(3.20)$, notamos que o intervalo $\Delta \eta_{0}=\left(\eta_{o}-\right.$ $\eta_{f}$ ) é a distância (comóvel) entre o observador e o ponto em que o fóton espalhou-se com um elétron pela última vez antes de ser observado. Como notado no Apêndice C, a identidade (3.20) anula-se identicamente a menos que $r, \Delta \eta_{1}, \ldots, \Delta \eta_{n}$ formem lados de um polígono fechado que tenha o lado $r$

\footnotetext{
${ }^{3} \mathrm{~A}$ integral que aparece em (3.14) é também expressa em termos de probabilidades associadas a vôos aleatórios, mas vôos com apenas um percurso. Como neste caso a densidade de probabilidade é trivialmente conhecida, verifica-se que para $n=1$ obtém-se de (C.21) o mesmo resultado estabelecido no Lema C.2.
} 

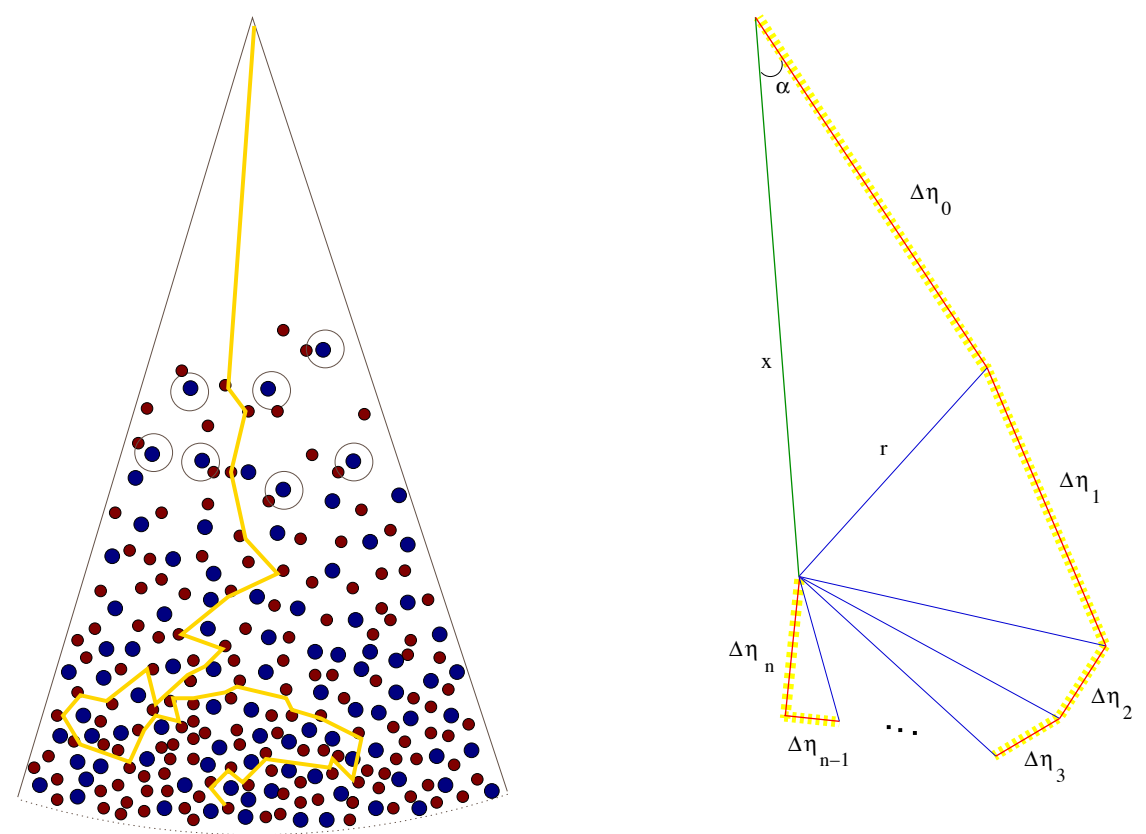

Fig. 3.2 Conexão entre espalhamentos durante a recombinação (esquerda) e diagrama de vôo aleatório (direita). Os trechos $\Delta \eta_{n}, \ldots, \Delta \eta_{1}$ fazem parte do vôo aleatório determinado pelos espalhamentos durante a recombinação. O trecho $\Delta \eta_{0}$ corresponde à propagação livre desde o último espalhamento durante a recombinação até sua observação. O evento de observação é representado pelo vértive superior dos diagramas.

em comum com um triângulo de lados $\Delta \eta_{0}, x$ e $r$. Esses vínculos geométricos restringem o domínio em que pode variar $x$ e, em especial, impõem um limite superior a esta variável: $x$ não pode ser superior a $\Delta \eta_{0}+\Delta \eta_{1}+\ldots+\Delta \eta_{n}=\left(\eta_{o}-\eta\right)$.

Uma tentativa de ilustrar a conexão descrita acima é a Figura 3.2 onde, na esquerda, apresenta-se pictoricamente uma seqüência de interações elétronfóton durante a recombinação, e na direita apresenta-se um diagrama representando os trechos do vôo aleatório associado à seqüência de espalhamentos. Cada trecho entre espalhamentos sucessivos durante a recombinação é associado a um intervalo $\Delta \eta$. Os trechos $\Delta \eta_{n}, \ldots, \Delta \eta_{1}$ fazem parte do vôo aleatório determinado pelos espalhamentos durante a recombinação. $\mathrm{O}$ trecho $\Delta \eta_{0}$ corresponde à propagação livre desde o último espalhamento durante a recombinação até sua observação. O lado $r$, como descrevemos, é comum ao triângulo $\Delta \eta_{0}, x, r$ e também fecha o polígono de lados $r, \Delta \eta_{1}, \ldots, \Delta \eta_{n}$. 
74 Equações de Boltzmann para temperatura e polarização da CMB no espaço das posições

\subsection{Retorno à descrição da polarização quando $P=0$}

Dadas as considerações sobre o problema dos vôos aleatórios que apresentamos e sua conexão com o problema de cosmologia que nos interessa, voltamos a (3.17) para escrever o termo geral da série dos $\pi_{l m}$ :

$$
\begin{aligned}
\pi_{l m}^{(n)}\left(\eta_{o}\right)= & -\frac{3}{4 \pi} \sqrt{\frac{(l+2) !}{(l-2) !}} \int_{0}^{\eta_{o}} d \eta_{f} \mu^{\prime}\left(\eta_{f}\right) \mathrm{e}^{-\mu\left(\eta_{f}\right)} \\
& \times \frac{1}{(n-1) !} \int_{0}^{\eta_{f}} d \eta^{\prime} \ldots d \eta_{i} \mathrm{~T}\left\{\mu^{\prime}\left(\eta^{\prime}\right) \mathrm{e}^{-\mu\left(\eta^{\prime}\right)} \ldots \mu^{\prime}\left(\eta_{i}\right) \mathrm{e}^{-\mu\left(\eta_{i}\right)}\right\} \\
& \times \frac{9^{(n-1)}\left(\frac{\pi}{2}\right)^{(n+1) / 2}}{(\Gamma(7 / 2))^{n-1}} \int_{0}^{\eta_{i}} d \eta \int_{0}^{\infty} d x x^{2} S_{l m}(x, \eta) \\
& \times \int d(\cos \alpha)\left(\frac{x \Delta \eta_{n}}{r^{3}}\right)^{2} P_{l}^{-2}(\cos \alpha) \operatorname{sen}^{2} \alpha p_{n}\left(r ; \Delta \eta_{1}, \ldots, \Delta \eta_{n} \mid 7\right) .(3.21)
\end{aligned}
$$

Podemos brevemente interpretar (3.21) da seguinte forma:

- A integral no ângulo $\alpha$ corresponde a uma marginalização sobre todos os possíveis caminhos formados de $n$ trechos de comprimentos $\Delta \eta_{1}, \ldots, \Delta \eta_{n}$ dados, e que satisfazem o vínculo de terem percorrido uma distância final fixada por $x$ e $\Delta \eta_{0}$ para todos os possíveis ângulos entre estes lados, como indicado na Figura 3.3. 


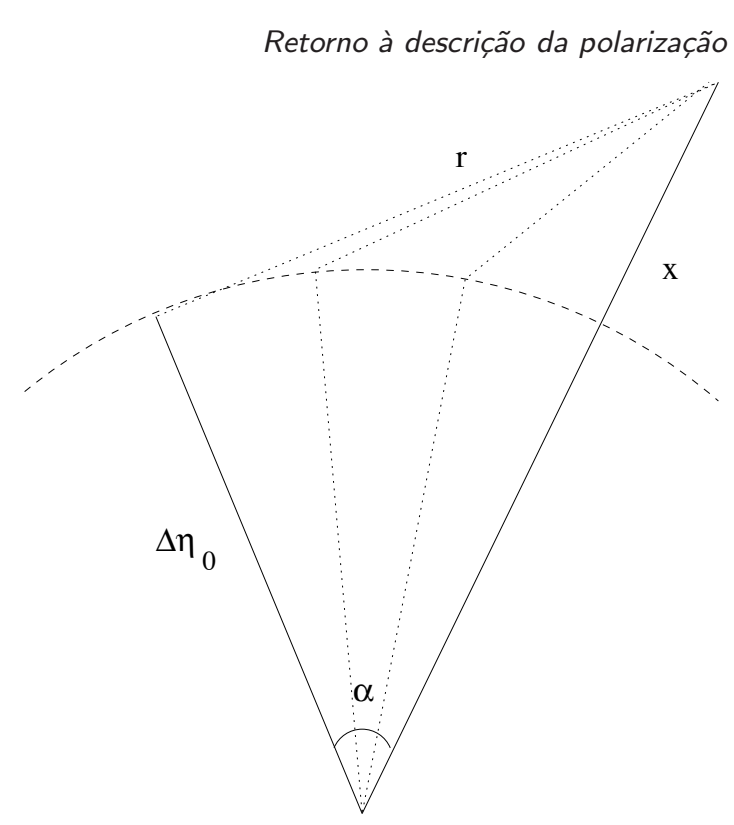

Fig. 3.3 Representação da marginalização sobre todos os possíveis caminhos formados de $n$ trechos de comprimentos $\Delta \eta_{1}, \ldots, \Delta \eta_{n}$ dados, e que satisfazem o vínculo de terem percorrido uma distância final fixada por $x$ e $\Delta \eta_{0}$ para todos os possíveis ângulos entre estes lados.

Naturalmente esta "média de caminhos"é função de $x, \Delta \eta_{0}, \Delta \eta_{1}, \ldots \Delta \eta_{n}$;

- A contribuição das fontes $S_{l m}(x, \eta)$ são computadas para a "média de caminhos" descrita acima para todos os possíveis valores de $\mathrm{x}$, sendo que o valor máximo que a variável $x$ pode assumir é $\Delta \eta_{0}+\Delta \eta_{1}+\ldots+\Delta \eta_{n}$. A contribuição das fontes calculadas sobre as médias de caminhos de $n$ trechos é ainda função de $\Delta \eta_{0}, \Delta \eta_{1}, \ldots, \Delta \eta_{n}$;

- Como último passo faz-se variar o tamanho dos intervalos $\Delta \eta_{0}, \Delta \eta_{1}, \ldots \Delta \eta_{n}$ através das integrações com as funções de visibilidade, fazendo com que a contribuição das fontes tenha sido tomada sobre todos os possíveis vôos aleatórios de $n$ trechos e todas as possíveis combinações de distâncias associadas a cada trecho do vôo.

A contribuição total de todas as ordens de iteração para o coeficiente da expansão da polarização pode ser escrita como: 
76 Equações de Boltzmann para temperatura e polarização da CMB no espaço das posições

$$
\begin{aligned}
\pi_{l m}\left(\eta_{o}\right)= & -\frac{3}{4 \pi} \sqrt{\frac{(l+2) !}{(l-2) !}} \int_{0}^{\eta_{o}} d \eta_{f} \mu^{\prime}\left(\eta_{f}\right) \mathrm{e}^{-\mu\left(\eta_{f}\right)} \\
& \times \sum_{n=1}^{\infty} \frac{1}{(n-1) !} \int_{0}^{\eta_{f}} d \eta^{\prime} \ldots d \eta_{i} \mathrm{~T}\left\{\mu^{\prime}\left(\eta^{\prime}\right) \mathrm{e}^{-\mu\left(\eta^{\prime}\right)} \ldots \mu^{\prime}\left(\eta_{i}\right) \mathrm{e}^{-\mu\left(\eta_{i}\right)}\right\} \\
& \times \frac{9^{(n-1)}\left(\frac{\pi}{2}\right)^{(n+1) / 2}}{(\Gamma(7 / 2))^{n-1}} \int_{0}^{\eta_{i}} d \eta \int_{0}^{\infty} d x x^{2} S_{l m}(x, \eta) \\
& \times \int d(\cos \alpha)\left(\frac{x \Delta \eta_{n}}{r^{3}}\right)^{2} P_{l}^{-2}(\cos \alpha) \operatorname{sen}^{2} \alpha p_{n}\left(r ; \Delta \eta_{1}, \ldots, \Delta \eta_{n} \mid 7\right) .
\end{aligned}
$$

\subsection{Diagramas}

Vamos agora introduzir uma representação diagramática para os termos que aparecem na expressão da temperatura e polarização em n-ésima ordem. Os elementos gráficos serão utilizados:

- Linhas sólidas representam o cálculo de polarização e linhas pontilhadas, temperatura;

- Linhas verticais mais à direita simbolizam o observável que está sendo calculado;

- A interseção de duas linhas determina quais as fontes são consideradas para o cálculo final de um observável.

As regras para a construção dos diagramas tornar-se-ão mais claras quando calcularmos, na sequência, uma série de termos explicitamente. A contribuição computada em (3.22), no entanto, pode ser expressa como:

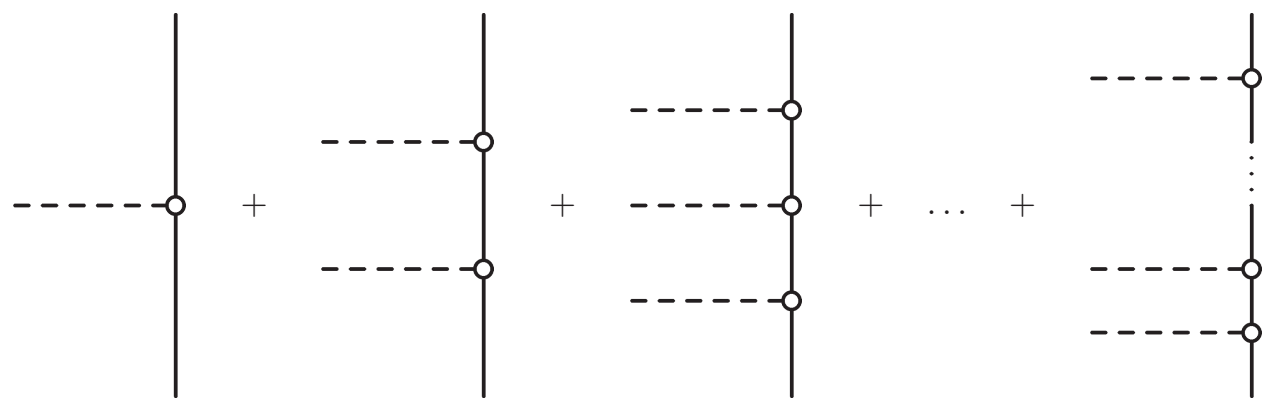




\subsection{Reintrodução de fontes de polarização na temperatura}

Retomaremos (3.1) e (3.2) mas agora mantendo todos os termos de fonte. A exemplo do que tínhamos em (3.7), buscando estabelecer uma decomposição como (3.6), escrevemos:

$$
\begin{aligned}
\theta_{l m}\left(\eta_{o}\right)= & 2 i^{l} \int_{0}^{\eta_{o}} d \eta \int \frac{d k}{(2 \pi)^{1 / 2}} k^{2}\left\{\left[\int d ^ { 2 } \hat { \mathbf { k } } \mathrm { e } ^ { - \mu ( \eta ) } \left(\mu ^ { \prime } ( \eta ) \left[\Theta_{S W}(\mathbf{k}, \eta)\right.\right.\right.\right. \\
& \left.\left.\left.-V_{b}(\mathbf{k}, \eta) \frac{\partial}{\partial \eta}\right]+\left(\Psi^{\prime}+\Phi^{\prime}\right)(\mathbf{k}, \eta)\right) \mathrm{Y}_{l m}^{*}(\hat{\mathbf{k}})\right] \\
& \left.+\frac{1}{2} \int d^{2} \hat{\mathbf{k}} \mathrm{e}^{-\mu(\eta)} \mu^{\prime}(\eta) P(\mathbf{k}, \eta)\left[1+3 \frac{\partial^{2}}{\partial\left(k\left(\eta_{o}-\eta\right)\right)^{2}}\right] \mathrm{Y}_{l m}^{*}(\hat{\mathbf{k}})\right\} j_{l}\left(k\left(\eta_{o}-\eta\right)\right) .
\end{aligned}
$$

O primeiro termo desta equação já foi tratado. Resta-nos portanto estudar o comportamento apenas do segundo termo, ou seja, aquele que contém o termo de fonte $P$. Assim escrevemos:

$$
\begin{aligned}
\theta_{l m}\left(\eta_{o}\right)= & \theta_{l m}^{(0)}\left(\eta_{o}\right)+i^{l} \int_{0}^{\eta_{o}} d \eta \int \frac{d k}{(2 \pi)^{1 / 2}} k^{2} \int d^{2} \hat{\mathbf{k}} \mathrm{e}^{-\mu(\eta)} \mu^{\prime}(\eta) P(\mathbf{k}, \eta) \mathrm{Y}_{l m}^{*}(\hat{\mathbf{k}}) \\
& \times\left[1+3 \frac{\partial^{2}}{\partial\left(k\left(\eta_{o}-\eta\right)\right)^{2}}\right] j_{l}\left(k\left(\eta_{o}-\eta\right)\right) \\
= & : \theta_{l m}^{(0)}\left(\eta_{o}\right)+\sum_{n=1}^{\infty} \theta_{l m}^{(n)}\left(\eta_{o}\right)
\end{aligned}
$$

\subsubsection{Temperatura em primeira ordem}

Tomando $P(\mathbf{k}, \eta)=\frac{1}{2} \Theta_{2}(\mathbf{k}, \eta)$ podemos calcular $\theta_{l m}^{(1)}$ :

$$
\begin{aligned}
\theta_{l m}^{(1)}\left(\eta_{o}\right)= & \frac{i^{l}}{2} \int_{0}^{\eta_{o}} d \eta_{s} \int \frac{d k}{(2 \pi)^{1 / 2}} k^{2} \int d^{2} \hat{\mathbf{k}} \mathrm{e}^{-\mu\left(\eta_{s}\right)} \mu^{\prime}\left(\eta_{s}\right) \Theta_{2}\left(\mathbf{k}, \eta_{s}\right) \mathrm{Y}_{l m}^{*}(\hat{\mathbf{k}}) \\
& \times\left[1+3 \frac{\partial^{2}}{\partial\left(k\left(\eta_{o}-\eta_{s}\right)\right)^{2}}\right] j_{l}\left(k\left(\eta_{o}-\eta_{s}\right)\right) \\
= & \frac{i^{l}}{2} \int_{0}^{\eta_{o}} d \eta_{s} \mathrm{e}^{-\mu\left(\eta_{s}\right)} \mu^{\prime}\left(\eta_{s}\right) \int \frac{d k}{(2 \pi)^{1 / 2}} k^{2} \int_{0}^{\eta_{s}} d \eta S_{l m}(k, \eta) j_{2}\left(k\left(\eta_{s}-\eta\right)\right) \\
& \times\left[1+3 \frac{\partial^{2}}{\partial\left(k\left(\eta_{o}-\eta_{s}\right)\right)^{2}}\right] j_{l}\left(k\left(\eta_{o}-\eta_{s}\right)\right) \\
= & \frac{1}{2 \pi} \int_{0}^{\eta_{o}} d \eta_{s} \mathrm{e}^{-\mu\left(\eta_{s}\right)} \mu^{\prime}\left(\eta_{s}\right) \int_{0}^{\eta_{s}} d \eta \int_{0}^{\infty} d x x^{2} S_{l m}(x, \eta) \\
\times & \int d k k^{2} j_{l}(k x)\left[1+3 \frac{\partial^{2}}{\partial\left(k\left(\eta_{o}-\eta_{s}\right)\right)^{2}}\right] j_{l}\left(k\left(\eta_{o}-\eta_{s}\right)\right) j_{2}\left(k\left(\eta_{s}-\eta\right)\right)
\end{aligned}
$$


78 Equações de Boltzmann para temperatura e polarização da CMB no espaço das posições onde utilizamos novamente a transformação dos coeficientes $S_{l m}(k, \eta)$ para o espaço das posições. Temos agora que tratar a integral:

$$
\int d k k^{2} j_{l}(k x)\left[1+3 \frac{\partial^{2}}{\partial\left(k\left(\eta_{o}-\eta_{s}\right)\right)^{2}}\right] j_{l}\left(k\left(\eta_{o}-\eta_{s}\right)\right) j_{2}\left(k\left(\eta_{s}-\eta\right)\right) .
$$

Claramente,

$$
\begin{aligned}
& \int d k k^{2} j_{l}(k x)\left[1+3 \frac{\partial^{2}}{\partial\left(k\left(\eta_{o}-\eta_{s}\right)\right)^{2}}\right] j_{l}\left(k\left(\eta_{o}-\eta_{s}\right)\right) j_{2}\left(k\left(\eta_{s}-\eta\right)\right) \\
& =\underbrace{\int d k k^{2} j_{l}(k x) j_{l}\left(k\left(\eta_{o}-\eta_{s}\right)\right) j_{2}\left(k\left(\eta_{s}-\eta\right)\right)}_{I_{1}} \\
& +3\left[\frac{\partial^{2}}{\partial\left(\eta_{o}-\eta_{s}\right)^{2}}-\frac{1}{\left(\eta_{o}-\eta_{s}\right)} \frac{\partial}{\partial\left(\eta_{o}-\eta_{s}\right)}\right] \underbrace{\int d k j_{l}(k x) j_{l}\left(k\left(\eta_{o}-\eta_{s}\right)\right) j_{2}\left(k\left(\eta_{s}-\eta\right)\right)}_{I_{2}} .
\end{aligned}
$$

A integral $I_{2}$ é do tipo calculada no Lema C.2. Já $I_{1}$ é do tipo (C.23) com $f(k)=k^{2}, n=1$ e $m=2$ e sabemos, portanto, que ela pode ser expressa em termos de integrais também do tipo $I_{2}$ através da relação (C.28). Explicitamente,

$$
\begin{aligned}
I_{1}= & \frac{1}{\left[\left(\eta_{o}-\eta_{s}\right) x\right]^{l+2}} \frac{\partial}{\partial x} \frac{\partial}{\partial\left(\eta_{o}-\eta_{s}\right)} \\
& \times\left\{\left[\left(\eta_{o}-\eta_{s}\right) x\right]^{l+2} \int d k j_{l+1}(k x) j_{l+1}\left(k\left(\eta_{o}-\eta_{s}\right)\right) j_{2}\left(k\left(\eta_{s}-\eta\right)\right)\right\}
\end{aligned}
$$

e daí calculada através do Lema C.2.

Procedendo aos cálculos, obtemos:

$$
\begin{aligned}
\theta_{l m}^{(1)}\left(\eta_{o}\right)= & \frac{1}{2} \int_{0}^{\eta_{o}} d \eta_{s} \mathrm{e}^{-\mu\left(\eta_{s}\right)} \mu^{\prime}\left(\eta_{s}\right) \int_{0}^{\eta_{s}} d \eta \int_{0}^{\infty} d x x^{2} S_{l m}(x, \eta) \\
& \times\left\{3\left[\frac{\partial^{2}}{\partial\left(\eta_{o}-\eta_{s}\right)^{2}}-\frac{1}{\left(\eta_{o}-\eta_{s}\right)} \frac{\partial}{\partial\left(\eta_{o}-\eta_{s}\right)}\right]\left[\frac{\left(\eta_{o}-\eta_{s}\right) x}{\left(\eta_{s}-\eta\right)^{3}} P_{l}^{-2}(\cos \alpha) \operatorname{sen}^{2} \alpha\right]\right. \\
& \left.+\frac{1}{\left[\left(\eta_{o}-\eta_{s}\right) x\right]^{l+2}} \frac{\partial}{\partial x} \frac{\partial}{\partial\left(\eta_{o}-\eta_{s}\right)}\left[\frac{\left[\left(\eta_{o}-\eta_{s}\right) x\right]^{l+3}}{\left(\eta_{s}-\eta\right)^{3}} P_{l+1}^{-2}(\cos \alpha) \operatorname{sen}^{2} \alpha\right]\right\}(3.27)
\end{aligned}
$$

Aqui $\alpha$ é o ângulo formado entre $\left(\eta_{o}-\eta_{s}\right)$ e $x$, que devem formar um triângulo junto com $\left(\eta_{s}-\eta\right)$. Se $\left(\eta_{o}-\eta_{s}\right),\left(\eta_{s}-\eta\right)$ e $x$ não formarem um triângulo, 
$\theta_{l m}^{(1)}\left(\eta_{o}\right)$ anula-se, o que mostra que também para a expansão da temperatura em termos de uma solução iterativa há respeito ordem a ordem das relações de causalidade com domínios de dependência bem definidos.

Podemos também expressar este resultado de maneira mais conveniente para a determinação de termos gerais como:

$$
\begin{aligned}
\theta_{l m}^{(1)}\left(\eta_{o}\right)= & \frac{1}{2} \int_{0}^{\eta_{o}} d \eta_{s} \mathrm{e}^{-\mu\left(\eta_{s}\right)} \mu^{\prime}\left(\eta_{s}\right) \int_{0}^{\eta_{s}} d \eta \int_{0}^{\infty} d x x^{2} S_{l m}(x, \eta) \\
& \times\left\{3\left[\frac{\partial^{2}}{\partial\left(\eta_{o}-\eta_{s}\right)^{2}}-\frac{1}{\left(\eta_{o}-\eta_{s}\right)} \frac{\partial}{\partial\left(\eta_{o}-\eta_{s}\right)}\right]\left[\left(\eta_{o}-\eta_{s}\right)^{2} H_{l 2}\left(\left(\eta_{o}-\eta_{s}\right), x ;\left(\eta_{s}-\eta\right)\right)\right]\right. \\
& +\frac{1}{\left(\eta_{o}-\eta_{s}\right)^{l} x^{l+2}} \frac{\partial}{\partial x}\left(\frac{\partial}{\partial\left(\eta_{o}-\eta_{s}\right)}+\frac{2}{\left(\eta_{o}-\eta_{s}\right)}\right)\left[\left[\left(\eta_{o}-\eta_{s}\right) x\right]^{l+2}\right. \\
& \left.\left.\times H_{(l+1) 2}\left(\left(\eta_{o}-\eta_{s}\right), x ;\left(\eta_{s}-\eta\right)\right)\right]\right\},
\end{aligned}
$$

onde usamos (C.28).

\subsubsection{Temperatura em segunda ordem}

Se queremos calcular as contribuições em segunda ordem para a temperatura, devemos incluir as contribuições para $\Theta$ que vem do quadrupolo da temperatura, ou seja, aquelas que vem de (3.28), como também aquelas que vem de $\alpha_{2}$, dadas em (3.14). As duas contribuições combinadas levam-nos a:

$$
\begin{aligned}
\theta_{l m}^{(2)}\left(\eta_{o}\right)= & \frac{1}{4 \pi} \int_{0}^{\eta_{o}} d \eta_{s} \mathrm{e}^{-\mu\left(\eta_{s}\right)} \mu^{\prime}\left(\eta_{s}\right) \int_{0}^{\eta_{s}} d \eta_{i} \mathrm{e}^{-\mu\left(\eta_{i}\right)} \mu^{\prime}\left(\eta_{i}\right) \int_{0}^{\eta_{i}} d \eta \int_{0}^{\infty} d x x^{2} S_{l m}(x, \eta) \\
& \times \int d k k^{2} j_{l}(k x) j_{2}\left(k\left(\eta_{i}-\eta\right)\right)\left[1+3 \frac{\partial^{2}}{\partial\left(k\left(\eta_{s}-\eta_{i}\right)\right)^{2}}\right] \\
& \times j_{2}\left(k\left(\eta_{s}-\eta_{i}\right)\right)\left[1+3 \frac{\partial^{2}}{\partial\left(k\left(\eta_{o}-\eta_{s}\right)\right)^{2}}\right] j_{l}\left(k\left(\eta_{o}-\eta\right)\right) \\
& +\frac{9}{\pi} \int_{0}^{\eta_{o}} d \eta_{s} \mathrm{e}^{-\mu\left(\eta_{s}\right)} \mu^{\prime}\left(\eta_{s}\right) \int_{0}^{\eta_{s}} d \eta_{i} \mathrm{e}^{-\mu\left(\eta_{i}\right)} \mu^{\prime}\left(\eta_{i}\right) \int_{0}^{\eta_{i}} d \eta \int_{0}^{\infty} d x x^{2} S_{l m}(x, \eta) \\
& \times \int d k k^{2} j_{l}(k x) j_{2}\left(k\left(\eta_{i}-\eta\right)\right) \frac{j_{2}\left(k\left(\eta_{s}-\eta_{i}\right)\right)}{\left(k\left(\eta_{s}-\eta_{i}\right)\right)^{2}} \\
& \times\left[1+3 \frac{\partial^{2}}{\partial\left(k\left(\eta_{o}-\eta_{s}\right)\right)^{2}}\right] j_{l}\left(k\left(\eta_{o}-\eta_{s}\right)\right) .
\end{aligned}
$$

O primeiro termo em (3.29) é a contribuição de $\Theta_{2}$ para $\theta_{l m}^{(2)}$. O segundo termo é a contribuição de $\alpha_{2}$. Ambas as contribuições podem ser expressas em termos da função $H_{l m}$ introduzida em (C.22): nos termos em que aparecem 
80 Equações de Boltzmann para temperatura e polarização da CMB no espaço das posições duas derivadas, contribuirá $H_{l 2}\left(\left(\eta_{o}-\eta_{s}\right), x,\left(\eta_{s}-\eta_{i}\right),\left(\eta_{i}-\eta\right)\right)$; nos termos em que aparece uma derivada, contribuirá $H_{(l+1) 2}\left(\left(\eta_{o}-\eta_{s}\right), x,\left(\eta_{s}-\eta_{i}\right),\left(\eta_{i}-\eta\right)\right)$ e nos termos em que não aparecem derivadas, teremos $H_{(l+2) 2}\left(\left(\eta_{o}-\eta_{s}\right), x, \eta_{s}-\right.$ $\left.\left.\eta_{i}\right),\left(\eta_{i}-\eta\right)\right)$.

A expansão completa até a segunda ordem dos coeficientes da temperatura - que será denotada por $\bar{\theta}_{l m}$ - pode ser diagramaticamente representada como:

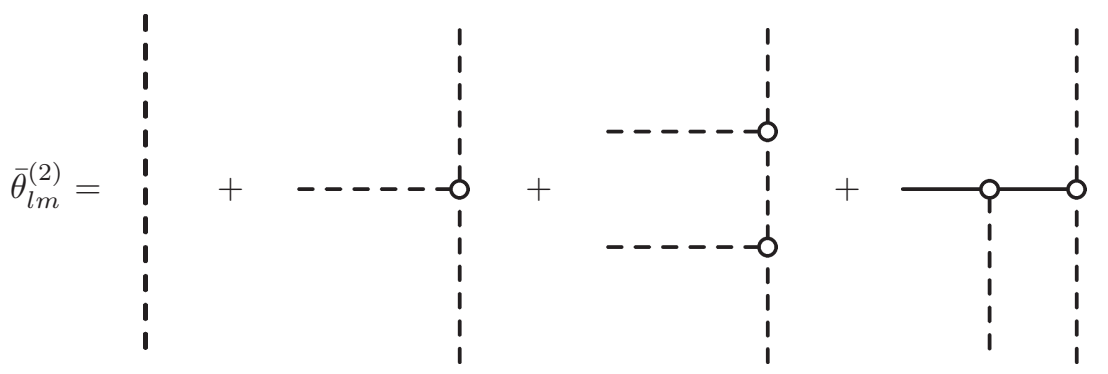

O primeiro diagrama representa $\theta_{l m}^{(0)}$, o segundo, $\theta_{l m}^{(1)}$ e os dois últimos os termos presentes em $\theta_{l m}^{(2)}$, como se pode ver em (3.29).

O entendimento dessa estrutura de composição dos termos que contribuem para o contraste de temperatura da CMB leva-nos a dar a seguinte interpretação aos termos:

- $\theta_{l m}^{(0)}$ corresponde ao termo mais simples, associado a fótons que se desacoplam e propagam-se até o observador sem sofrer nenhum espalhamento ao longo de suas trajetórias;

- $\theta_{l m}^{(1)}$ corresponde a fótons que se espalharam uma única vez entre o desacoplamento e a observação. Antes do espalhamento os fótons eram não polarizados, e após o espalhamento tornam-se parcialmente polarizados. Ainda que esse efeito não seja relevante para o estudo apenas da temperatura, deve-se notar que a intensidade total de radiação observada corresponde a uma composição de uma fração não-polarizada e uma fração polarizada. A fração não-polarizada, no entanto, é afetada pela existência de um espalhamento prévio.

- $\theta_{l m}^{(2)}$ corresponde a fótons que se espalharam duas vezes entre o desacoplamento e a observação. No primeiro trecho os fótons são não-polarizados, e se tornam parcialmente polarizados após o primeiro espalhamento. Esses fótons que se espalharam uma vez, vão espalhar-se uma segunda vez e por isso têm uma intensidade conjunta que é a soma das intensidades da 
fração de fótons que permaneceram não-polarizados após o espalhamento e da fração que se polarizou - e daí a presença de $\alpha_{2}^{(2)}$ como fonte para a temperatura na hierarquia das equações de Boltzmann.

O resultado final da consideração de todos os termos é somar todas as possíveis combinações de efeitos advindos de todos os possíveis percursos percorríveis pelos fótons compondo frações de intensidade polarizada e não-polarizada, donde se pode admirar a complexidade da solução da hierarquia de Boltzmann.

Mesmo que não calculemos explicitamente as contribuições em todas as ordens, os resultados apresentados na Seção C.2 mostram que todas as possíveis correções podem ser expressas em termos de combinações de funções $H_{l m}$. Em geral, podemos escrever

$$
\bar{\theta}_{l m}^{(n)}=\sum_{q=0}^{n} \theta_{l m q}^{(n)}\left(\Delta \eta_{0}, \Delta \eta_{1}, \ldots, \Delta \eta_{n}, x\right) H_{(l+q) 2}\left(\Delta \eta_{0}, x, \Delta \eta_{1}, \ldots, \Delta \eta_{n}\right),
$$

onde $\theta_{l m q}^{(n)}$ são os coeficientes que aparecerão na expansão. Como se pode ver explicitamente no caso $n=1$, os coeficientes são combinações de operadores diferenciais e potências dos subintervalos presentes na partição de $\left(\eta_{o}-\eta\right)$ e $x$. A expressão completa de $\bar{\theta}_{l m}^{(n)}$ depende também do conhecimento da polarização em ordem $n-1$.

\subsubsection{Polarização em segunda ordem}

Vamos agora analisar o primeiro caso em que efeitos corretivos na temperatura são utilizados para o cálculo da polarização. Vamos escrever a polarização em segunda ordem como:

$$
\begin{aligned}
\bar{\pi}_{l m}^{(2)}= & -\frac{3}{2} \sqrt{\frac{(l+2) !}{(l-2) !}} \int_{0}^{\eta_{o}} d \eta_{s} \mathrm{e}^{-\mu\left(\eta_{s}\right)} \mu^{\prime}\left(\eta_{s}\right) \int \frac{d^{3} \mathbf{k}}{(2 \pi)^{3 / 2}} 4 \pi i^{l} \frac{1}{2} \Theta_{2}\left(\mathbf{k}, \eta_{s}\right) \mathrm{Y}_{l m}^{*}(\hat{\mathbf{k}}) \frac{j_{l}\left(k\left(\eta_{o}-\eta_{s}\right)\right)}{\left(k\left(\eta_{o}-\eta_{s}\right)\right)^{2}} \\
= & -\frac{3}{2} i^{l} \sqrt{\frac{(l+2) !}{(l-2) !}} \int_{0}^{\eta_{o}} d \eta_{s} \mathrm{e}^{-\mu\left(\eta_{s}\right)} \mu^{\prime}\left(\eta_{s}\right)\left\{\int_{0}^{\eta_{s}} d \eta \int \frac{d k}{(2 \pi)^{1 / 2}} k^{2} S_{l m}(k, \eta) j_{2}\left(k\left(\eta_{s}-\eta\right)\right)\right. \\
& +\frac{1}{2} \int_{0}^{\eta_{s}} d \eta_{i} \mathrm{e}^{-\mu\left(\eta_{i}\right)} \mu^{\prime}\left(\eta_{i}\right) \int \frac{d k}{(2 \pi)^{1 / 2}} k^{2} \int d^{2} \hat{\mathbf{k}} P\left(\mathbf{k}, \eta_{i}\right) \mathrm{Y}_{l m}^{*}(\hat{\mathbf{k}}) \\
& \left.\times\left[1+3 \frac{\partial^{2}}{\partial\left(k\left(\eta_{s}-\eta_{i}\right)\right)^{2}}\right] j_{2}\left(k\left(\eta_{s}-\eta_{i}\right)\right)\right\} \frac{j_{l}\left(k\left(\eta_{o}-\eta_{s}\right)\right)}{\left(k\left(\eta_{o}-\eta_{s}\right)\right)^{2}}
\end{aligned}
$$

onde a barra sobrescrita em $\bar{\pi}_{l m}^{(2)}$ indica que estamos tomando em conta todos os efeitos até a segunda ordem. 
82 Equações de Boltzmann para temperatura e polarização da CMB no espaço das posições

Notamos que destaca-se entre os termos a contribuição de primeira ordem $\pi_{l m}^{(1)}$. Como queremos o efeito mais baixo de correções da temperatura que influencia a polarização, tomaremos apenas a contribuição de $\Theta_{2}$ para $P$ :

$$
\begin{aligned}
\bar{\pi}_{l m}^{(2)}= & \pi_{l m}^{(1)}-\frac{3}{4} i^{l} \sqrt{\frac{(l+2) !}{(l-2) !}} \int_{0}^{\eta_{o}} d \eta_{s} \mathrm{e}^{-\mu\left(\eta_{s}\right)} \mu^{\prime}\left(\eta_{s}\right) \int_{0}^{\eta_{s}} d \eta_{i} \mathrm{e}^{-\mu\left(\eta_{i}\right)} \mu^{\prime}\left(\eta_{i}\right) \int \frac{d k}{(2 \pi)^{1 / 2}} k^{2} \\
& \times \int d^{2} \hat{\mathbf{k}} \Theta_{2}\left(\mathbf{k}, \eta_{2}\right) \mathrm{Y}_{l m}^{*}(\hat{\mathbf{k}})\left[1+3 \frac{\partial^{2}}{\partial\left(k\left(\eta_{s}-\eta_{i}\right)\right)^{2}}\right] j_{2}\left(k\left(\eta_{s}-\eta_{i}\right)\right) \frac{j_{l}\left(k\left(\eta_{o}-\eta_{s}\right)\right)}{\left(k\left(\eta_{o}-\eta_{s}\right)\right)^{2}} \\
= & \pi_{l m}^{(1)}-\frac{3}{4} i^{l} \sqrt{\frac{(l+2) !}{(l-2) !}} \int_{0}^{\eta_{o}} d \eta_{s} \mathrm{e}^{-\mu\left(\eta_{s}\right)} \mu^{\prime}\left(\eta_{s}\right) \int_{0}^{\eta_{s}} d \eta_{i} \mathrm{e}^{-\mu\left(\eta_{i}\right)} \mu^{\prime}\left(\eta_{i}\right) \int \frac{d k}{(2 \pi)^{1 / 2}} k^{2} \\
& \times \int_{0}^{\eta_{i}} d \eta S_{l m}(k, \eta) j_{2}\left(k\left(\eta_{i}-\eta\right)\right)\left[1+3 \frac{\partial^{2}}{\partial\left(k\left(\eta_{s}-\eta_{i}\right)\right)^{2}}\right] j_{2}\left(k\left(\eta_{s}-\eta_{i}\right)\right) \frac{j_{l}\left(k\left(\eta_{o}-\eta_{s}\right)\right)}{\left(k\left(\eta_{o}-\eta_{s}\right)\right)^{2}} \\
= & \pi_{l m}^{(1)}-\frac{1}{2} \frac{3}{2 \pi} \sqrt{\frac{(l+2) !}{(l-2) !}} \int_{0}^{\eta_{o}} d \eta_{s} \mathrm{e}^{-\mu\left(\eta_{s}\right)} \mu^{\prime}\left(\eta_{s}\right) \int_{0}^{\eta_{s}} d \eta_{i} \mathrm{e}^{-\mu\left(\eta_{i}\right)} \mu^{\prime}\left(\eta_{i}\right) \int_{0}^{\eta_{i}} d \eta \int d x x^{2} S_{l m}(x, \eta) \\
& \times\left\{\int d k k^{2} j_{l}(k x) \frac{j_{l}\left(k\left(\eta_{o}-\eta_{s}\right)\right)}{\left(k\left(\eta_{o}-\eta_{s}\right)\right)^{2}}\left[1+3 \frac{\partial^{2}}{\partial\left(k\left(\eta_{s}-\eta_{i}\right)\right)^{2}}\right] j_{2}\left(k\left(\eta_{s}-\eta_{i}\right)\right) j_{2}\left(k\left(\eta_{i}-\eta\right)\right)\right\}(3.32)
\end{aligned}
$$

A integral entre chaves pode ser reexpressa com a ajuda de (C.28) como:

$$
\begin{aligned}
& \int d k k^{2} j_{l}(k x) \frac{j_{l}\left(k\left(\eta_{o}-\eta_{s}\right)\right)}{\left(k\left(\eta_{o}-\eta_{s}\right)\right)^{2}}\left[1+3 \frac{\partial^{2}}{\partial\left(k\left(\eta_{s}-\eta_{i}\right)\right)^{2}}\right] j_{2}\left(k\left(\eta_{s}-\eta_{i}\right)\right) j_{2}\left(k\left(\eta_{i}-\eta\right)\right) \\
& =\frac{\left(\left(\eta_{s}-\eta_{i}\right)\right)^{2}}{\left(\left(\eta_{o}-\eta_{s}\right) x\right)^{l+2}} \frac{\partial}{\partial x}\left(\frac{\partial}{\partial\left(\eta_{o}-\eta_{s}\right)}+\frac{2}{\left(\eta_{o}-\eta_{s}\right)}\right) \\
& \times\left[\left(\left(\eta_{o}-\eta_{s}\right) x\right)^{l+2} H_{(l+1) 2}\left(\left(\eta_{o}-\eta_{s}\right), x ;\left(\eta_{s}-\eta_{i}\right),\left(\eta_{i}-\eta\right)\right)\right] \\
& +3\left(\frac{\partial^{2}}{\partial\left(\eta_{s}-\eta_{i}\right)^{2}}-\frac{1}{\left(\eta_{s}-\eta_{i}\right)} \frac{\partial}{\partial\left(\eta_{s}-\eta_{i}\right)}\right) \\
& \times\left[\left(\eta_{s}-\eta_{i}\right)^{2} H_{l 2}\left(\left(\eta_{s}-\eta_{i}\right), x ;\left(\eta_{s}-\eta_{i}\right),\left(\eta_{i}-\eta\right)\right)\right] .
\end{aligned}
$$

Como podemos ver, apesar da presença de coeficientes complicados, o resultado final pode ser expresso em termos de combinações das funções $H_{l 2}\left(\left(\eta_{o}-\right.\right.$ $\left.\left.\eta_{s}\right), x ;\left(\eta_{s}-\eta_{i}\right),\left(\eta_{i}-\eta\right)\right)$ e $H_{(l+1) 2}\left(\left(\eta_{o}-\eta_{s}\right), x ;\left(\eta_{s}-\eta_{i}\right),\left(\eta_{i}-\eta\right)\right)$.

O último termo em (3.32) tem a mesma ordem que $\pi_{l m}^{(2)}$. Assim, incluindo todas as correções de até segunda ordem, a polarização é dada pela soma dos três seguintes diagramas: 


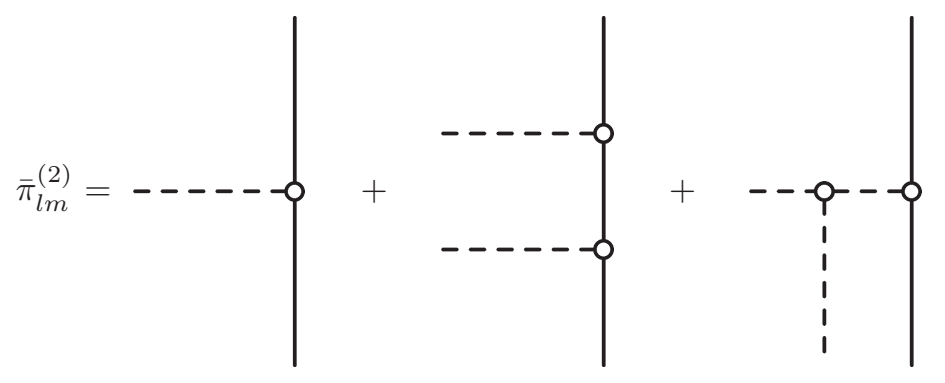

Podemos também expressar formalmente (3.32) como:

$$
\begin{aligned}
\bar{\pi}_{l m}^{(2)}= & \pi_{l m 0}\left(\left(\eta_{o}-\eta_{s}\right),\left(\eta_{s}-\eta_{i}\right),\left(\eta_{i}-\eta\right), x\right) H_{l 2}\left(\left(\eta_{o}-\eta_{s}\right), x ;\left(\eta_{s}-\eta_{i}\right),\left(\eta_{i}-\eta\right)\right) \\
& +\pi_{l m 1}\left(\left(\eta_{o}-\eta_{s}\right),\left(\eta_{s}-\eta_{i}\right),\left(\eta_{i}-\eta\right) ; x\right) H_{(l+1) 2}\left(\left(\eta_{o}-\eta_{s}\right), x ;\left(\eta_{s}-\eta_{i}\right),\left(\eta_{i}-\eta\right)\right) .
\end{aligned}
$$

Os coeficientes $\pi_{l m q}(q=0,1)$ são operadores diferenciais e dependem de potências de combinações de subintervalos que compõem a partição do intervalo $\eta_{o}-\eta$. O coeficiente $\pi_{l m 0}$ codifica as contribuições de $\pi_{l m}^{(1)}$ tal como aparece em (3.32) e também a contribuição do segundo termo no lado direito da equação (3.33). O coeficiente $\pi_{l m 1}$ expressa o primeiro termo no lado direito da equação (3.33).

Se examinamos (3.32) e (3.34), vemos que $\bar{\pi}_{l m}^{(2)}$ é dado pela soma de $\pi_{l m}^{(2)}$ com a contribuição para a polarização da temperatura uma vez corrigida por si mesma. Poderíamos escrever (3.34) de uma maneira mais simétrica notando que os diagramas a seguir são equivalentes:

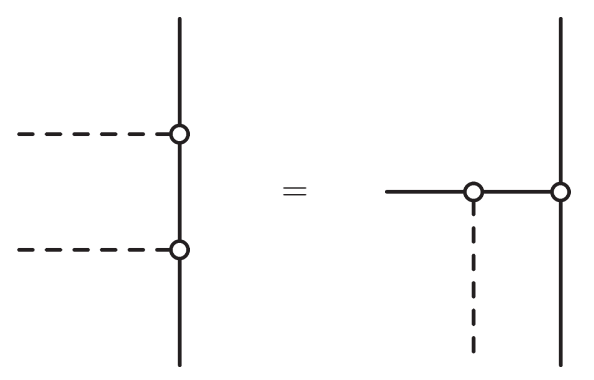

A equivalência desses diagramas é devida ao fato de ambos terem o mesmo tipo de linha vertical à direita (ou seja, estamos calculando polarização), ambos têm o mesmo número de vértices, o mesmo tipo de linha que conecta os vértices e, finalmente, ambos têm a mesma fonte, ou seja, temperatura (linhas tracejadas). 
84 Equações de Boltzmann para temperatura e polarização da CMB no espaço das posições Em geral, para ordem $n$ :

$$
\bar{\pi}_{l m}^{(n)}=\sum_{q=0}^{n-1} \pi_{l m q}^{(n)}\left(\Delta \eta_{0}, \Delta \eta_{1}, \ldots, \Delta \eta_{n}, X\right) H_{(l+q) 2}\left(\Delta \eta_{0}, X ; \Delta \eta_{1}, \ldots, \Delta \eta_{n}\right) .
$$

As fórmulas explícitas para os coeficientes $\pi_{l m q}^{(n)}$ não são conhecidas, mas podem ser computadas uma vez que se determina até que ordem pretende-se tomar em conta os efeitos corretivos. O procedimento para a determinação desses coeficientes é aquele apresentado na Seção C.2.

Naturalmente o cálculo de $\bar{\pi}_{l m}^{(n)}$ requer o conhecimento de $\bar{\theta}_{l m}^{(n-1)}$. O cálculo de $\bar{\theta}_{l m}^{(n-1)}$ requer, por sua vez, o conhecimento tanto de $\bar{\theta}_{l m}^{(n-2)}$ quanto de $\bar{\pi}_{l m}^{(n-2)}$, e assim sucessivamente. Claramente os coeficientes formam uma hierarquia.

\subsubsection{Regras para a construção de diagramas}

Como indicamos no cálculo de alguns casos em mais baixa ordem, o conjunto de equações de Boltzmann definido por (3.1), (3.2), (3.3) and (3.4) pode ter sua solução dada, uma vez fixada uma ordem de precisão, através de uma expansão no número de espalhamentos sofridos por fótons durante a recombinação ${ }^{4}$. Termos representando uma determinada ordem $n$ na expansão trarão densidades de probabilidade associadas a vôos aleatórios compostos de $n$ segmentos. As diferentes contribuições de correções de temperatura à polarização e polarização à temperatura podem ser representadas através de diagramas. As regras gerais para construção de diagramas são apresentadas na tabela 3.1.

Como regra prática, as funções $j_{l}(k x)$ e $j_{2}\left(k\left(\eta_{n}-\eta\right)\right)$ devem aparecer como primeiro e último termo na integral em $k$. Aqui, $\eta_{n}$ é o último índice na partição do intervalo $\left(\eta_{o}-\eta\right)$. Os intervalos $\Delta$ devem ser substituídos pela diferença de tempos conformes consecutivos convenientes e que representem os instantes inicial e final de um segmento de vôo aleatório. A integral espacial $\int d x x^{2} S_{l m}(x, \eta)$ deve também sempre comparecer. Finalmente, devem-se incluir as integrais temporais e os respectivos pesos dados pela função de visibilidade. A última integral temporal - integral em $\eta$ - não carrega a função de visibilidade.

\footnotetext{
${ }^{4}$ Naturalmente que quando se começa a demandar precisão progressivamente maior na solução da hierarquia, pode-se perguntar se efeitos de segunda ordem nas perturbações da métrica não teriam a mesma ordem de contribuição [57]. Neste caso uma extensão do formalismo seria necessária.
} 


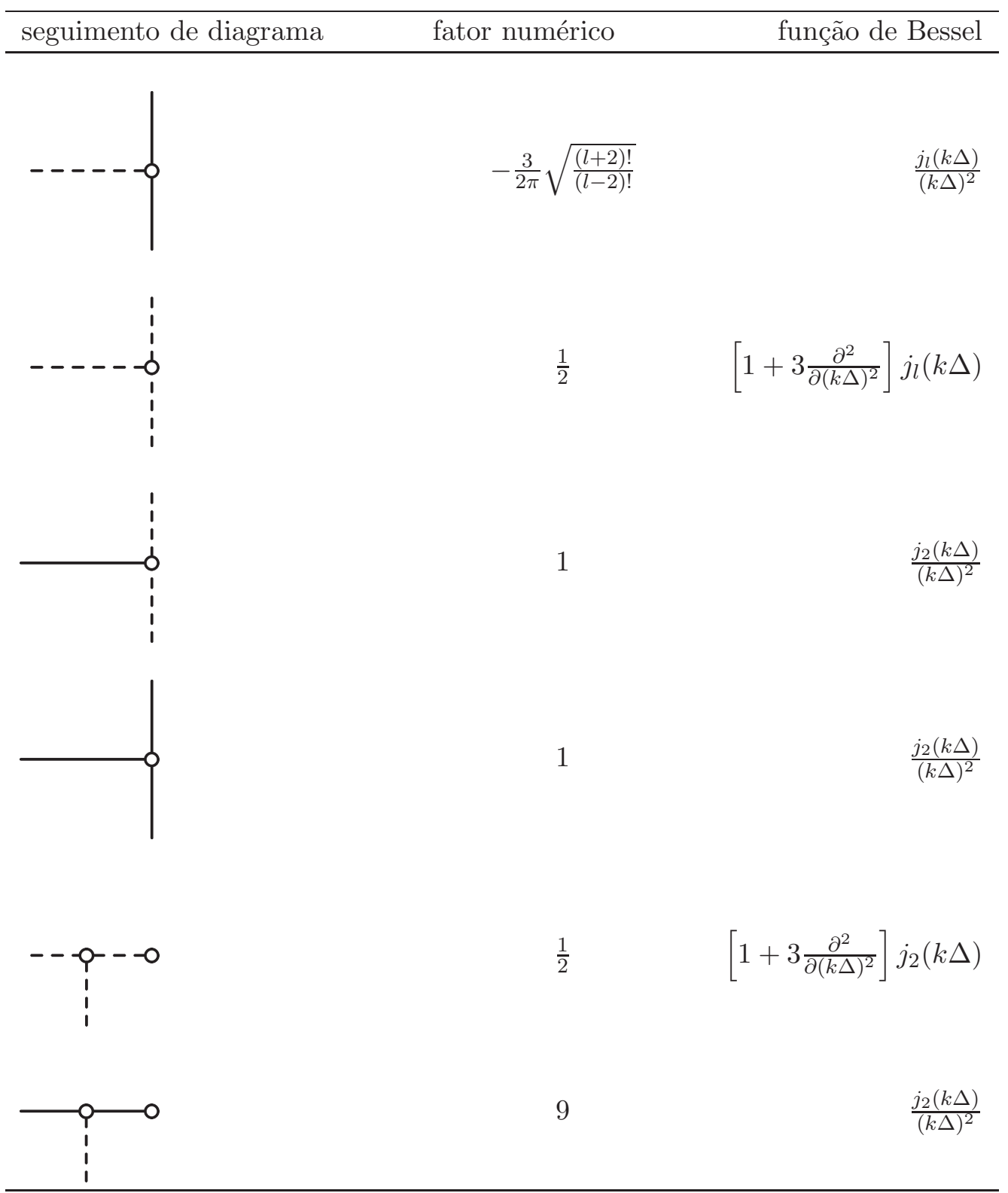

Table 3.1 Blocos constitutivos de diagramas em geral.

Como exemplo, vamos calcular dois diagramas: o primeiro representa uma contribuição de terceira ordem para a polarização, tomando em conta o termo da polarização em segunda ordem advindo da auto-correção da temperatura: 


$$
\begin{aligned}
& =\frac{9}{2} \frac{3}{2 \pi} \sqrt{\frac{(l+2) !}{(l-2) !}} \int_{0}^{\eta_{o}} d \eta_{s} \mathrm{e}^{-\mu\left(\eta_{s}\right)} \mu^{\prime}\left(\eta_{s}\right) \int_{0}^{\eta_{s}} d \eta_{i} \mathrm{e}^{-\mu\left(\eta_{i}\right)} \mu^{\prime}\left(\eta_{i}\right) \\
& \times \int_{0}^{\eta_{i}} d \eta_{f} \mathrm{e}^{-\mu\left(\eta_{f}\right)} \mu^{\prime}\left(\eta_{f}\right) \int_{0}^{\eta_{f}} d \eta \\
& \times \int d x x^{2} S_{l m}(x, \eta)\left\{\int d k k^{2} j_{l}(k x) \frac{j_{2}\left(k\left(\eta_{o}-\eta_{s}\right)\right)}{\left(k\left(\eta_{o}-\eta_{s}\right)\right)^{2}} \frac{j_{2}\left(k\left(\eta_{s}-\eta_{i}\right)\right.}{\left(k\left(\eta_{s}-\eta_{i}\right)\right)^{2}}\right. \\
& \left.\times\left[1+3 \frac{\partial^{2}}{\partial\left(k\left(\eta_{i}-\eta_{f}\right)\right)^{2}}\right] j_{2}\left(k\left(\eta_{i}-\eta_{f}\right)\right) j_{2}\left(k\left(\eta_{f}-\eta\right)\right)\right\} .
\end{aligned}
$$

Já o diagrama abaixo mostra a contribuição de terceira ordem para a polarização vinda da inserção da temperatura auto-corrigida duas vezes:

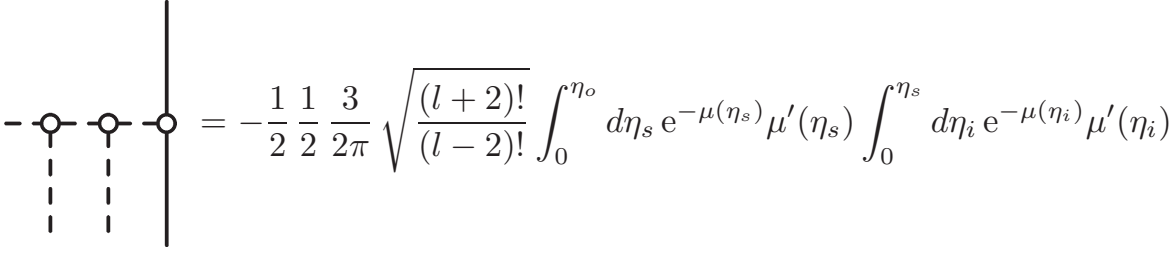

$$
\begin{aligned}
& \times \int_{0}^{\eta_{i}} d \eta_{f} \mathrm{e}^{-\mu\left(\eta_{f}\right)} \mu^{\prime}\left(\eta_{f}\right) \int_{0}^{\eta_{f}} d \eta \\
& \times \int d x x^{2} S_{l m}(x, \eta)\left\{\int d k k^{2} j_{l}(k x) \frac{j_{l}\left(k\left(\eta_{o}-\eta_{s}\right)\right)}{\left(k\left(\eta_{o}-\eta_{s}\right)\right)^{2}}\right. \\
& \times\left[1+3 \frac{\partial^{2}}{\partial\left(k\left(\eta_{s}-\eta_{i}\right)\right)^{2}}\right] j_{2}\left(k\left(\eta_{s}-\eta_{i}\right)\right) \\
& \left.\times\left[1+3 \frac{\partial^{2}}{\partial\left(k\left(\eta_{i}-\eta_{f}\right)\right)^{2}}\right] j_{2}\left(k\left(\eta_{i}-\eta_{f}\right)\right) j_{2}\left(k\left(\eta_{f}-\eta\right)\right)\right\} \text {. }
\end{aligned}
$$

\subsection{Domínios de dependência}

Estudaremos como a presença de densidades de probabilidade associadas a vôos aleatórios determina os domínios de dependência para a hierarquia de equações de Boltzmann que estamos considerando. Como notamos no Apêndice C, e é bem entendido na literatura - [53], por exemplo - a interpretação para $p_{n}\left(r ; r_{1}, \ldots, r_{n} \mid D\right)$ é a probabilidade de que após um vôo aleatório em $D$ dimensões composto de $n$ trechos de comprimentos $r_{1}, r_{2}, \ldots, r_{n}$, o caminhante 
tenha percorrido uma distância $r$. Caso a soma $r_{1}+r_{2}+\ldots+r_{n}$ seja inferior a $r$, não há hipótese de que o caminhante possa ter percorrido uma distância igual a $r$ após os $n$ trechos fixados, e portanto a probabilidade neste caso é nula. Há portanto um limite superior para $r$, dado em termos dos comprimentos de vôos livres antes de mudanças de direção do caminhante. Com efeito, a trajetória do vôo aleatório é uma poligonal sendo $r$ o afastamento desde a origem do movimento. De acordo com as construções apresentadas no Apêndice C, para que as integrais de produtos de funções de Bessel esféricas com que nos deparamos sejam definidas, é necessário também que $r$ seja determinado em termos do afastamento do observador ao último ponto alcançado pelo vôo aleatório (que denotamos por $\left.\left(\eta_{o}-\eta_{f}\right)\right)$ e a variável de integração $x$. De fato, é necessário que $\left(\eta_{o}-\eta_{f}\right), x$ e $r$ formem um triângulo.

A síntese das restrições geométricas impostas pelo vôo aleatório e pela condição de que $\left(\eta_{o}-\eta_{f}\right), x$ e $r$ formem um triângulo é que as fontes são sempre computadas no cone de luz passado do espalhamento mais remoto tomado em consideração em uma dada ordem fixa. As mesmas restrições de domínio de dependência são observadas para temperatura e polarização.

Um conhecido teorema de relatividade geral [58] afirma que se $\gamma$ é uma geodésica nula ligando pontos $a$ e $b$, e $\beta$ é uma geodésica nula ligando pontos $b$ e $c$, então ou $\gamma \cup \beta$ é uma geodésica nula entre $a$ e $c$ ou há uma curva tipo-tempo que liga $a$ e $c$. No contexto de nosso estudo esse teorema aplica-se completamente: no caso em que não há espalhamentos entre emissão e observação dos fótons, a variável de integração $x$ é exatamente restrita sobre o cone de luz, como se pode ver em (3.11). Com a presença de espalhamentos a variável $x$ pode estar sobre o cone de luz passado do observador - no caso de não haver mudanças de direção do fóton nos espalhamentos - ou no interior do cone de luz passado do observador, sendo a direção por ela definida uma curva tipo-tempo ligando os pontos de emissão e de observação do fóton.

Apenas como uma nota final sobre o assunto, devemos observar que não é surpreendente que um processo relacionado a vôos aleatórios tenha domínio de dependência bem definido, por estarem associados a equações diferenciais parciais hiperbólicas. Com efeito, [59] mostrou que, em uma dimensão, densidades de probabilidade descrevendo a posição do caminhante executando vôos aleatórios são descritas por uma equação de telegrafista que é, sabidamente, hiperbólica. A relação entre vôos aleatórios e equações hiperbólicas é geral, 
88 Equações de Boltzmann para temperatura e polarização da CMB no espaço das posições embora, como notado por [60], exemplos explícitos de equações de difusão hiperbólicas são mais uma exceção que uma regra.

\subsection{Possível forma de cálculo das densidades de probabilidade}

Até agora mostramos que a hierarquia de Boltzmann para a temperatura e polarização da CMB pode ser reconstruída em termos de médias das fontes sobre caminhos correspondentes a vôos aleatórios que correspondem à história de fótons na recombinação. As densidades de probabilidade que surgem nesse tratamento não são analiticamente conhecidas e correspondem, na verdade, um problema aberto na teoria de probabilidades (ver, por exemplo, [61] ou [62]). As densidades de probabilidade são, contudo, expressas em termos de integrais que poderiam ser numericamente calculadas. Propomos, entretanto, que há uma maneira mais eficiente de calcular estas probabilidades associadas a caminhos aleatórios, a saber, através de expansões de Fourier-Bessel ${ }^{5}$.

Para mostrar como essas probabilidades podem ser calculadas em termos de uma série, devemos identificar a última integral em (3.22) como o caso de $m=2$ da expressão (C.38). Em conseqüência desta identificação, (C.38) poderia ser tomada como uma forma de calcular a referida integral em (3.22), calculando $p_{n}\left(r ; \Delta \eta_{1}, \ldots, \Delta \eta_{n} \mid 7\right)$ através de uma soma de seus modos de decomposição em série de Fourier-Bessel.

Esta, entretanto, ainda não é a melhor forma de calcular o valor da integral que desejamos por dois motivos: primeiramente resta ainda em (C.38) uma integral. Em segundo lugar a normalização das raízes dos zeros das funções de Bessel esféricas é feita em termos da soma $\Delta \eta_{1}+\ldots+\Delta \eta_{n}$ ficando de fora o trecho $\Delta \eta_{0}$ que, embora não corresponda a um trecho entre espalhamentos dentro do vôo aleatório, é uma variável que desejaríamos tratar de uma forma mais próxima que $\Delta \eta_{1}, \ldots, \Delta \eta_{n}$.

Tomamos, então, a expressão equivalente (C.40), isto é,

$$
\begin{aligned}
& \int d(\cos \alpha)\left(\frac{x \Delta \eta_{n}}{r^{3}}\right)^{2} P_{l}^{-2}(\cos \alpha) \operatorname{sen}^{2} \alpha p_{n}\left(r ; \Delta \eta_{1}, \ldots, \Delta \eta_{n} \mid 7\right) \\
= & \sum_{q=1}^{\infty} \frac{\pi}{\tilde{a}^{3} j_{l+1}^{2}\left(y_{l q} \tilde{a}\right)} \frac{j_{l}\left(y_{l q} \Delta \eta_{0}\right)}{\left(y_{l q} \Delta \eta_{0}\right)^{2}} j_{l}\left(y_{l q} x\right) \frac{j_{2}\left(y_{l q} \Delta \eta_{1}\right)}{\left(y_{l q} \Delta \eta_{1}\right)^{2}} \frac{j_{2}\left(y_{l q} \Delta \eta_{2}\right)}{\left(y_{l q} \Delta \eta_{2}\right)^{2}} \ldots \frac{j_{2}\left(y_{l q} \Delta \eta_{n-1}\right)}{\left(y_{l q} \Delta \eta_{n-1}\right)^{2}} j_{2}\left(y_{l q} \Delta \eta_{n}\right)
\end{aligned}
$$

${ }^{5}$ Esse procedimento não constitui uma novidade no estudo de processos envolvendo vôos aleatórios. De fato, [63] cita um trabalho de 1947 que faz este tipo de tratamento para tratar integrais de vôo aleatório. A referência é W. R. Bennet, Q. Appl. Math. 5, 385, (1947). 
onde

$$
y_{l q}=\frac{\alpha_{q}^{l}}{\tilde{a}}
$$

$\alpha_{q}^{l}$ é o q-ésimo zero da função de Bessel esférica de ordem $l$, e $\tilde{a}=\left(\eta_{o}-\eta\right)=$ $\Delta \eta_{0}+\Delta \eta_{1}+\ldots+\Delta \eta_{n}$.

Espera-se que a qualidade de convergência da série apresentada forneça um método adequado ao cálculo numérico da integral de nosso interesse.

\subsection{Considerações sobre convergência}

Tomemos em consideração a expressão (3.22), que descreve os coeficientes da expansão da polarização. Quanto maior a ordem do termo que se deseja tomar em conta, menor deve ser a contribuição, já que cada potência da função de visibilidade acarreta, por exemplo, potências da seção de choque de espalhamentos Thomson. Temos daí a intuição de que termos mais altos da expansão devem contribuir pouco. Buscamos aqui analisar esse mesmo ponto sob outro aspecto: vamos supor que o sistema elétrons-fótons durante a recombinação esteja ainda interagente o suficiente para que um livre caminho médio possa ser definido como constante (o que é uma aproximação, naturalmente). Se o sistema é interagente, é razoável assumir que entre o início da recombinação até a propagação livre do fóton tenha havido um número grande de espalhamentos $^{6}$. Em resumo, queremos estudar $p_{n}\left(r ; \Delta \eta_{1}, \Delta \eta_{2}, \ldots, \Delta \eta_{n} \mid 7\right)$ quando $n$ é grande e $\Delta \eta_{1}=\Delta \eta_{2}=\ldots=\Delta \eta_{n}=: \Delta$.

A referência [53] mostra, através do método de Laplace, que $P_{n}(r ; \Delta, \Delta, \ldots, \Delta \mid 7)$, no limite desejado, ou seja, $n \rightarrow \infty$, pode-se escrever como:

$$
P_{n}(r ; \Delta, \Delta, \ldots, \Delta \mid 7)=\frac{1}{\Gamma(9 / 2)}\left(\frac{7 r^{2}}{2 n \Delta^{2}}\right)^{7 / 2}{ }_{1} F_{1}\left(\frac{7}{2} ; \frac{9}{2} ; \frac{-7 r^{2}}{2 n \Delta}\right),
$$

ou seja, a probabilidade de que após $n$ trechos de comprimento $\Delta$ o caminhante esteja a uma distância menor ou igual a $r$ pode ser expressa em termos de uma função hipergeométrica confluente.

Lembrando que [64]

$$
{ }_{1} F_{1}(a ; c ; z)=\frac{\Gamma(c)}{\Gamma(a)} \sum_{k=0}^{\infty} \frac{\Gamma(a+k)}{\Gamma(c+k)} \frac{z^{k}}{k !}
$$

\footnotetext{
${ }^{6} \mathrm{~A}$ aproximação aqui apresentada é equivalente a tomar a função de visibilidade como uma constante dentro de um determinado intervalo em torno da época de recombinação, e zero fora desse intervalo.
} 


$$
\frac{d}{d z}{ }_{1} F_{1}(a ; c ; z)=\frac{a}{c}{ }_{1} F_{1}(a+1 ; c+1 ; z)
$$

podemos mostrar inicialmente que

$$
\begin{aligned}
p_{n}(r ; \Delta, \ldots, \Delta \mid 7)= & \frac{1}{\Gamma(7 / 2)}\left(\frac{7 r^{2}}{2 n \Delta^{2}}\right)^{5 / 2}\left(\frac{7 r}{2 n \Delta^{2}}\right)\left[{ }_{1} F_{1}\left(\frac{7}{2} ; \frac{9}{2} ; \frac{-7 r^{2}}{2 n \Delta^{2}}\right)\right. \\
& \left.-\left(\frac{7 r^{2}}{9 n \Delta^{2}}\right){ }_{1} F_{1}\left(\frac{9}{2} ; \frac{11}{2} ; \frac{-7 r^{2}}{2 n \Delta^{2}}\right)\right]
\end{aligned}
$$

e depois, através da expansão em série de potências de ${ }_{1} F_{1}$, que:

$$
\begin{aligned}
p_{n}(r ; \Delta, \ldots, \Delta \mid 7)= & \frac{7 / 2}{\Gamma(7 / 2)}\left(\frac{7 r^{2}}{2 n \Delta^{2}}\right)^{5 / 2}\left(\frac{7 r}{2 n \Delta^{2}}\right) \\
& \times \sum_{k=0}^{\infty} \frac{1}{k !} \frac{\left(\frac{9}{2}-\frac{7 r^{2}}{2 n \Delta^{2}}\right)+k\left(1-\frac{r^{2}}{n \Delta^{2}}\right)}{\left(\frac{7}{2}+k\right)\left(\frac{9}{2}+k\right)}\left(\frac{-7 r^{2}}{2 n \Delta^{2}}\right)^{k} .
\end{aligned}
$$

Consideremos então a soma:

$$
S_{z}=\sum_{k=0}^{\infty} \frac{1}{k !} \frac{\left(\frac{9}{2}-z\right)+k\left(1-\frac{2}{7} z\right)}{\left(\frac{7}{2}+k\right)\left(\frac{9}{2}+k\right)}(-z)^{k} \leq \sum_{k=0}^{\infty} \frac{1}{k !} \frac{1}{\left(\frac{7}{2}+k\right)}(-z)^{k} \leq \mathrm{e}^{-z},
$$

que se traduz na desigualdade:

$$
p_{n}(r ; \Delta, \ldots, \Delta \mid 7) \leq \frac{1}{r}\left(\frac{7 r^{2}}{2 n \Delta^{2}}\right)^{7 / 2} \mathrm{e}^{-\frac{7 r^{2}}{2 n \Delta^{2}}} .
$$

Isso mostra que, para $r$ e $\Delta$ fixos, $r \leq n \Delta$,

$$
\lim _{n \rightarrow \infty} p_{n}(r ; \Delta, \ldots, \Delta \mid 7)=0 .
$$

Como $p_{n}$ é uma distribuição de probabilidades normalizada, concluímos que a densidade de probabilidades colapsa, neste limite, a um delta de Dirac centrado na origem, o que concorda com as características gerais das distribuições de probabilidade associadas a vôos aleatórios [65]. Em outros termos, aumentar o número de espalhamentos em um vôo aleatório acarreta em trajetórias com afastamentos cada vez menores da origem.

Podemos avaliar as consequências deste fato sobre a polarização através de (3.22). Consideremos para isso a integral: 


$$
\begin{aligned}
\mathcal{S}_{l m} & =\lim _{n \rightarrow \infty} \int_{0}^{\eta_{o}-\eta} d x x^{2} S_{l m}(x, \eta) \int d(\cos \alpha)\left(\frac{x \Delta}{r^{3}}\right)^{2} P_{l}^{-2}(\cos \alpha)(\operatorname{sen} \alpha)^{2} p_{n}(r ; \Delta, \ldots, \Delta \mid 7) \\
& =\int_{0}^{\eta_{o}-\eta} d x x^{2} S_{l m}(x, \eta) \int d r \frac{r}{x \Delta \eta_{0}}\left(\frac{x \Delta}{r^{3}}\right)^{2} P_{l}^{-2}(\cos \alpha)(\operatorname{sen} \alpha)^{2} \delta(r) \\
& =\int_{0}^{\eta_{o}-\eta} d x x^{2} S_{l m}(x, \eta) \frac{\Delta^{2}}{\left(\Delta \eta_{0}\right)^{2}} \lim _{r \rightarrow 0}\left[\frac{x \Delta \eta_{0}}{r^{5}} P_{l}^{-2}(\cos \alpha)(\operatorname{sen} \alpha)^{2}\right]
\end{aligned}
$$

Sabemos, entretanto, do Lema C.2, que:

$$
\int_{0}^{\infty} d k j_{l}\left(k r_{1}\right) j_{l}\left(k r_{2}\right) j_{2}\left(k r_{3}\right)=\frac{\pi}{4} \frac{r_{1} r_{2}}{r_{3}^{3}} P_{l}^{-2}(\cos \alpha)(\operatorname{sen} \alpha)^{2},
$$

e que [4]:

$$
\lim _{r_{3} \rightarrow 0} \int_{0}^{\infty} d k j_{l}\left(k r_{1}\right) j_{l}\left(k r_{2}\right) j_{2}\left(k r_{3}\right)=\frac{r_{2}^{2}}{r_{1}^{4}} \frac{r_{3}^{2}}{15} \frac{\pi}{2} \delta\left(r_{1}-r_{2}\right) .
$$

Segue então que:

$$
\begin{aligned}
\mathcal{S}_{l m}\left(\eta_{0}, \eta\right) & =\int_{0}^{\eta_{0}-\eta} d x x^{2} S_{l m}(x, \eta) \frac{\pi}{30} \frac{\Delta^{2}}{x^{4}} \delta\left(\Delta \eta_{0}-x\right) \\
& =\frac{\pi}{30} \frac{\Delta^{2}}{\left(\Delta \eta_{0}\right)^{2}} S_{l m}\left(\Delta \eta_{0}, \eta\right) .
\end{aligned}
$$

A lição é que no limite de um número muito grande de espalhamentos, temos essencialmente o mesmo que se passaria em uma recombinação instantânea, havendo ainda a importante supressão devida ao fator $\left(\Delta / \Delta \eta_{0}\right)^{2} \rightarrow 0$. A conclusão, portanto, é que no limite de infinitos espalhamentos não há polarização gerada. Um argumento similar aplica-se a flutuações da temperatura.

Esse resultado é suficiente para mostrar que o peso a ser atribuído a caminhos aleatórios compostos de muitos trechos de tamanho fixo é naturalmente diminuto pela própria natureza das distribuições de probabilidade. Com efeito, $p_{n}(r ; \Delta, \ldots, \Delta \mid 7)$ expressa no limite em que estamos estudando será máxima quando $n=(r / \Delta)^{2}$, ou seja, no limite difusivo. A caminhos não-difusivos atribui-se probabilidades progressivamente menores.

O limite que estudamos é, naturalmente, bastante artificial, mas conveniente por conhecermos um expressão analítica aproximada para as probabilidades de vôos aleatórios. Apesar da artificialidade, esse exemplo tem duas grandes qualidades: primeiro, indica que ainda que as funções de visibilidade possam estar todas em seu máximo, há esperança de convergência da solução iterativa (ainda que estejamos falando em termos absolutamente informais). A segunda razão 
92 Equações de Boltzmann para temperatura e polarização da CMB no espaço das posições de interesse desse limite - e diretamente associada à primeira qualidade descrita - é indicar que, à medida que se amplia o número de interações fótons-elétrons, há progressiva diminuição da polarização líquida dos fótons, indicando que dinamicamente satisfaz-se o limite requerido para a maximização da entropia de von Neumann associada a sistemas de fótons em direção ao equilíbrio. Da mesma maneira, os efeitos de distorção sobre a distribuição de temperatura diminuem à medida em que se aproxima de um estado de equilíbrio, como esperaríamos do teorema $\mathrm{H}$ de Boltzmann [27]. 


\section{Efeito de lentes fracas sobre a temperatura e polarização da CMB: estudo no espaço das posições}

O desenvolvimento até agora apresentado resultou na forma integral das equações de Boltzmann no espaço das posições para a descrição da temperatura e polarização da radiação cósmica de fundo. Elemento central nesta descrição é, com efeito, o conhecimento das geodésicas nulas do espaço FLRW que permite relacionar dois pontos do espaço-tempo $\left(\mathbf{x}_{s}\right.$ e $\left.\mathbf{x}_{o}\right)$ como:

$$
\mathbf{x}_{s}=\mathbf{x}_{o}+\mathbf{e}\left(\eta_{o}-\eta_{s}\right)
$$

onde e é a direção em que se observa um fóton e $\left(\eta_{o}-\eta_{s}\right)$, a diferença de tempos conformes que representa a distância comóvel entre os pontos considerados.

Sabemos que as lentes gravitacionais serão responsáveis por alterações na correspondência da direção em que se observa um dado fóton e sua real direção de procedência. Nosso objetivo agora é introduzir o efeito das lentes gravitacionais, através do mapa de lente, na forma integral das equações de Boltzmann.

\subsection{Tratamento da temperatura}

Como havíamos discutido na Seção 2.8.4, no regime de lentes fracas é possível escrever, em termos do potencial de lente, um mapa bastante simples relacionando a direção de observação de um fóton e sua real direção de procedência especificamente, o fazemos através das equações (2.68) e (2.70). Desde que não hajam pontos conjugados ou pontos de corte ao longo de nenhuma geodésica nula caracterizando a trajetória de algum dos fótons em que nos interessemos, o ângulo de correção entre as direções de origem real do fóton e aquele distorcido por lentes deve ser também pequeno. Neste caso propõe-se que o efeito das lentes gravitacionais sobre a temperatura da CMB possa ser descrito através de uma expansão de Taylor em torno da direção real de procedência do fóton $[48,49]$ : 
94 Efeito de lentes fracas sobre a temperatura e polarização da CMB: estudo no espaço das posições

$$
\Theta\left(\eta_{o}, \tilde{\mathbf{e}}\right)=\Theta\left(\eta_{o}, \mathbf{e}+\nabla \psi\right)
$$

onde ẽ é a direção em que se observa o fóton após o efeito das lentes gravitacionais e e é a direção em que o observaríamos caso as lentes não interviessem. $\psi$ é o potencial de lente. Supondo fraco o efeito das lentes, e consequentemente pequeno o ângulo de desvio, propomos que seja acurada a expansão:

$$
\Theta\left(\eta_{o}, \tilde{\mathbf{e}}\right)=\Theta\left(\eta_{o}, \mathbf{e}+\nabla \psi\right) \approx \Theta\left(\eta_{o}, \mathbf{e}\right)+\nabla^{c} \psi \nabla_{c} \Theta\left(\eta_{o}, \mathbf{e}\right) .
$$

O contraste de temperatura é dado por:

$$
\Theta\left(\eta_{o}, \mathbf{e}\right)=\underbrace{\int \frac{d^{3} \mathbf{k}}{(2 \pi)^{3 / 2}} 4 \pi \sum_{l m} i^{l} \Theta_{l}\left(\mathbf{k}, \eta_{o}\right) \mathrm{e}^{i \mathbf{k} \cdot \mathbf{x}_{o}} \mathrm{Y}_{l m}^{*}(\hat{\mathbf{k}})}_{\theta_{l m}\left(\eta_{o}\right)} \mathrm{Y}_{l m}(\mathbf{e}) .
$$

Se escrevemos

$$
\sqrt{\frac{4 \pi}{2 l+1}} \mathrm{Y}_{l m}^{*}(\beta, \alpha)=D_{m 0}^{l}(\alpha, \beta, \gamma)
$$

com $\gamma=0$, podemos escrever a expansão de $\Theta$ em termos dos elementos de matriz da representação unitária irredutível do grupo $\mathrm{SO}(3)$. A estrutura de variedade do grupo de Lie $\mathrm{SO}(3)$ engendra uma forte interconexão entre objetos presentes no estudo de propriedades geométricas da esfera $S^{2}$ e objetos tipicamente associados ao estudo das representações de $\mathrm{SO}(3)$. Um exemplo desta interconexão - descrito no Apêndice D - é o fato de que operadores de derivação covariante sobre a esfera podem ser escritos, numa base conveniente, em termos de operadores que naturalmente surgem no estudo das representações unitárias irredutíveis do grupo de rotações, ð e $\bar{\partial}$. Estes operadores têm ação bem conhecida sobre os elementos da representação e são paralelos aos operadores $L_{+}$e $L_{-}$amplamente aplicados nos estudos da teoria do momento angular em mecânica quântica. A ação de ð e ठ̄ sobre $D_{m m^{\prime}}^{l}$ é apresentada no Apêndice D. Por estarem também ðे e $\bar{\partial}$ associados à derivada covariante, é possível fazer a expansão de Taylor que havíamos proposto na forma: 


$$
\begin{aligned}
\Theta\left(\eta_{o}, \tilde{\mathbf{e}}\right) & =\Theta\left(\eta_{o}, \mathbf{e}\right)+\nabla^{c} \psi \nabla_{c} \Theta\left(\eta_{o}, \mathbf{e}\right) \\
& =\Theta\left(\eta_{o}, \mathbf{e}\right)+\nabla^{+} \psi \nabla_{+} \Theta\left(\eta_{o}, \mathbf{e}\right)+\nabla^{-} \psi \nabla_{-} \Theta\left(\eta_{o}, \mathbf{e}\right) \\
& =\Theta\left(\eta_{o}, \mathbf{e}\right)-\nabla_{\mathbf{e}_{-}} \psi \nabla_{\mathbf{e}_{+}} \Theta\left(\eta_{o}, \mathbf{e}\right)-\nabla_{\mathbf{e}_{+}} \psi \nabla_{\mathbf{e}_{-}} \Theta\left(\eta_{o}, \mathbf{e}\right) \\
& =\Theta\left(\eta_{o}, \mathbf{e}\right)+\frac{1}{2} \bar{\partial} \psi \partial \Theta\left(\eta_{o}, \mathbf{e}\right)+\frac{1}{2} \precsim \psi \bar{\partial} \Theta\left(\eta_{o}, \mathbf{e}\right) \\
& =\Theta\left(\eta_{o}, \mathbf{e}\right)+\frac{1}{2}\left[\bar{\partial} \psi \partial \Theta\left(\eta_{o}, \mathbf{e}\right)+ð \psi \bar{\partial} \Theta\left(\eta_{o}, \mathbf{e}\right)\right] .
\end{aligned}
$$

Já que

$$
\nabla^{+}=g^{+c} \nabla_{c}=\underbrace{g^{++}}_{0} \nabla_{+}+\underbrace{g^{+-}}_{-1} \nabla_{-}=-\nabla_{-},
$$

com $g$ sendo a métrica escrita na base de vetores esféricos descrita no Apêndice D. Como os operadores $\check{\partial}$ e $\overline{\widetilde{\partial}}$ atuam apenas sobre a parte angular, ou seja, sobre as funções $D_{m m^{\prime}}^{l}$ aumentando ou diminuindo $m^{\prime}$ (como descrito nas equações (D.26) e (D.27)), obtemos:

$$
\begin{aligned}
\partial \Theta\left(\eta_{o}, \mathbf{e}\right) & =\sum_{l m} \theta_{l m}\left(\eta_{o}\right) \partial\left(\sqrt{\frac{2 l+1}{4 \pi}} D_{m 0}^{l *}(\mathbf{e})\right) \\
& =\sum_{l m} \sqrt{\frac{2 l+1}{4 \pi}} \theta_{l m}\left(\eta_{o}\right) \sqrt{l(l+1)} D_{m(-1)}^{l *}(\mathbf{e}), \\
\bar{\partial} \Theta\left(\eta_{o}, \mathbf{e}\right) & =-\sum_{l m} \sqrt{\frac{2 l+1}{4 \pi}} \theta_{l m}\left(\eta_{o}\right) \sqrt{l(l+1)} D_{m 1}^{l *}(\mathbf{e}) .
\end{aligned}
$$

Fazendo também a decomposição do potencial de lente como $\psi=\sum_{l m} \psi_{l m} \mathrm{Y}_{l m}=$ $\sum_{l m} \psi_{l m} \sqrt{\frac{2 l+1}{4 \pi}} D_{m 0}^{l *}$, podemos calcular:

$$
ð \psi=\sum_{l m} \sqrt{\frac{2 l+1}{4 \pi}} \psi_{l m} \sqrt{l(l+1)} D_{m(-1)}^{l *}
$$

e

$$
\bar{\jmath} \psi=-\sum_{l m} \sqrt{\frac{2 l+1}{4 \pi}} \psi_{l m} \sqrt{l(l+1)} D_{m 1}^{l *} .
$$

Podemos agora avaliar (4.4). Faremos isso em parcelas. Primeiramente consideremos: 
96 Efeito de lentes fracas sobre a temperatura e polarização da CMB: estudo no espaço das posições

$$
\begin{aligned}
\bar{\partial} \psi ð \Theta= & -\sum_{l m} \sum_{l^{\prime} m^{\prime}} \frac{\sqrt{(2 l+1)\left(2 l^{\prime}+1\right)}}{4 \pi} \sqrt{l l^{\prime}(l+1)\left(l^{\prime}+1\right)} \psi_{l^{\prime} m^{\prime}} \theta_{l m} D_{m^{\prime} 1}^{l^{\prime} *} D_{m(-1)}^{l *} \\
= & -\sum_{l m} \sum_{l^{\prime} m^{\prime}} \underbrace{\frac{1}{4 \pi} \sqrt{(2 l+1)\left(2 l^{\prime}+1\right) l l^{\prime}\left(l^{\prime}+1\right)(l+1)}}_{Z_{l l^{\prime}}} \psi_{l^{\prime} m^{\prime}} \theta_{l m} \\
& \times \sum_{L M M^{\prime}}(-1)^{m+m^{\prime}}\left\langle l^{\prime}-m^{\prime} l-m \mid L M\right\rangle\left\langle L M^{\prime} \mid l^{\prime}-1 l 1\right\rangle D_{M M^{\prime}}^{L} \\
= & -\sum_{L=0}^{\infty} \sum_{M=-L}^{L} \sum_{l=1}^{\infty} \sum_{l^{\prime}=1}^{\infty} \sum_{m=-l}^{l} Z_{l l^{\prime}} \psi_{l^{\prime}(-M-m)} \theta_{l m} \\
& \times(-1)^{M}\left\langle l^{\prime}(M+m) l-m \mid L M\right\rangle\left\langle L 0 \mid l^{\prime}-1 l 1\right\rangle D_{M 0}^{L}
\end{aligned}
$$

onde usamos regras de composição para os elementos de matrizes de Wigner e propriedades dos coeficientes de Clebsch-Gordan reunidas na Seção D.4 do Apêndice D.

De modo semelhante,

$$
\begin{aligned}
ð \psi \bar{\varnothing} \Theta= & -\sum_{L} \sum_{l m} \sum_{l^{\prime} m^{\prime}} \frac{1}{4 \pi} \sqrt{(2 l+1)\left(2 l^{\prime}+1\right) l l^{\prime}(l+1)\left(l^{\prime}+1\right)} \psi_{l^{\prime} m^{\prime}} \theta_{l m} \\
& \times \sum_{L M M^{\prime}}(-1)^{m+m^{\prime}}\left\langle l^{\prime}-m^{\prime} l-m \mid L M\right\rangle\left\langle L M^{\prime} \mid l^{\prime} 1 l-1\right\rangle D_{M M^{\prime}}^{L} \\
= & -\sum_{L=0}^{\infty} \sum_{M=-L}^{L} \sum_{l=1}^{\infty} \sum_{l^{\prime}=1}^{\infty} \sum_{m=-l}^{l} Z_{l l^{\prime}} \psi_{l^{\prime}(-M-m)} \theta_{l m} \\
& \times(-1)^{M}\left\langle l^{\prime}(M+m) l-m \mid L M\right\rangle\left\langle L 0 \mid l^{\prime} 1 l-1\right\rangle D_{M 0}^{L} .
\end{aligned}
$$

Portanto,

$$
\begin{aligned}
\Theta\left(\eta_{o}, \tilde{\mathbf{e}}\right)= & \Theta\left(\eta_{o}, \mathbf{e}\right)-\frac{1}{2} \sum_{L=0}^{\infty} \sum_{M=-L}^{L}\left\{\sum_{l m} \sum_{l^{\prime}=|l-L|}^{l+L}\left[\psi_{l^{\prime}(-M-m)} \theta_{l m}\right]\left(\eta_{o}\right)(-1)^{M}\left\langle l^{\prime}(M+m) l-m \mid L M\right\rangle\right. \\
& \left.\times\left[Z_{l l^{\prime}}\left\langle L 0 \mid l^{\prime}-1 l 1\right\rangle+Z_{l l^{\prime}}\left\langle L 0 \mid l^{\prime} 1 l-1\right\rangle\right]\right\} D_{M 0}^{L}(\mathbf{e}) \\
= & \Theta\left(\eta_{o}, \mathbf{e}\right)-\frac{1}{2} \sum_{L=0}^{\infty} \sum_{M=-L}^{L} \Xi_{L M}\left(\eta_{o}\right) \mathrm{Y}_{L M}(\mathbf{e}),
\end{aligned}
$$

onde definimos os coeficientes $\Xi_{L M}$ como: 


$$
\begin{aligned}
\Xi_{L M}\left(\eta_{o}\right)= & \sum_{l m} \sum_{l^{\prime}=|l-L|}^{l+L} \sqrt{\frac{4 \pi}{2 L+1}}\left[\psi_{l^{\prime}(M-m)} \theta_{l m}\right]\left(\eta_{o}\right)\left\langle l^{\prime}(-M+m) l-m \mid L-M\right\rangle \\
& \times Z_{l l^{\prime}}\left\langle L 0 \mid l^{\prime}-1 l 1\right\rangle\left[1+(-1)^{l+l^{\prime}-L}\right] .
\end{aligned}
$$

Em breve esclareceremos o significado de $\left[\psi_{l^{\prime}(M-m)} \theta_{l m}\right]\left(\eta_{o}\right)$.

Finalmente escrevemos:

$$
\Theta\left(\eta_{o}, \tilde{\mathbf{e}}\right)=\sum_{L=0}^{\infty} \sum_{M=-L}^{L}\left(\theta_{L M}\left(\eta_{o}\right)-\frac{1}{2} \Xi_{L M}\left(\eta_{o}\right)\right) \mathrm{Y}_{L M}(\mathbf{e}) .
$$

Vemos que esta expressão permite calcular o novo contraste de temperatura não apenas como uma correção ao antigo, mas mais especificamente como uma correção a seus coeficientes em uma expansão em harmônicos esféricos.

\subsection{Tratamento da polarização}

Vamos agora repetir essencialmente os mesmos passos que executamos acima para tomar em conta do efeito das lentes sobre a polarização da CMB. Devemos ter em mente, entretanto, que a polarização difere da temperatura por ser expressa em termos de uma expansão em uma base de harmônicos esféricos de spin. Vamos denotar por um momento, para simplificar a escrita:

$$
P\left(\eta_{o}, \mathbf{e}\right):=\frac{Q+i U}{4 I}\left(\eta_{o}, \mathbf{e}\right) .
$$

Da mesma maneira que observamos para o caso da temperatura, seja $P\left(\eta_{o}, \tilde{\mathbf{e}}\right)$ a função de polarização de fótons vindos de uma direção e após terem sofrido os efeitos de lentes gravitacionais durante sua propagação. Usando o mapa de lente (2.68), escrevemos:

$$
P\left(\eta_{o}, \tilde{\mathbf{e}}\right)=P\left(\eta_{o}, \mathbf{e}+\nabla \psi\right) .
$$

Supondo o efeito de lentes suficientemente fraco, podemos esperar que uma expansão de Taylor em torno da direção e em primeira ordem possa ser suficientemente acurada:

$$
P\left(\eta_{o}, \tilde{\mathbf{e}}\right)=P\left(\eta_{o}, \mathbf{e}+\nabla \psi\right) \approx P\left(\eta_{o}, \mathbf{e}\right)+\nabla^{c} \psi \nabla_{c} P\left(\eta_{o}, \mathbf{e}\right)
$$

sendo $c$ um índice sobre variáveis angulares. 
98 Efeito de lentes fracas sobre a temperatura e polarização da CMB: estudo no espaço das posições

Diferentemente da temperatura, o campo de polarização não é puramente escalar. Com efeito, como descrito no Apêndice D, o campo de polarização adquire uma fase quando se executam rotações em torno do eixo de observação, ou seja, de uma direção radial com relação à esfera celeste. Este comportamento sob rotações implica que a polarização tenha que ser decomposta em termos de harmônicos esféricos de spin. O campo de polarização tem, de fato, duas componentes, uma de spin positivo e outra de spin negativo. Tomemos, por exemplo, $P^{++}$[tendo incluído seus "índices de spin" de acordo com (D.10)] e façamos o mesmo que foi feito para a temperatura:

$$
\begin{aligned}
\tilde{P}^{++} & =P^{++}+\nabla^{c} \psi \nabla_{c} P^{++} \\
& =P^{++}+\nabla^{+} \psi \nabla_{+} P^{++}+\nabla^{-} \psi \nabla_{-} P^{++} \\
& =P^{++}-\nabla_{\mathbf{e}_{-}} \psi \nabla_{\mathbf{e}_{+}} P^{++}-\nabla_{\mathbf{e}_{+}} \psi \nabla_{\mathbf{e}_{-}} P^{++} \\
& =P^{++}+\frac{1}{2} \bar{\partial} \psi \precsim P^{++}+\frac{1}{2} \precsim \psi \bar{\partial} P^{++} \\
& =P^{++}+\frac{1}{2}\left(\bar{\partial} \psi \partial P^{++}+\check{\partial} \psi \bar{\partial} P^{++}\right) .
\end{aligned}
$$

Relembremos que:

$$
\frac{Q+i U}{4 I}\left(\eta_{o}, \mathbf{e}\right)=\sum_{l m} \underbrace{\left(\int \frac{d^{3} \mathbf{k}}{(2 \pi)^{3 / 2}} 4 \pi i^{l} \alpha_{l}^{(2)}\left(\mathbf{k}, \eta_{o}\right) \mathrm{Y}_{l m}^{*}(\hat{\mathbf{k}}) \mathrm{e}^{i \mathbf{k} \cdot \mathbf{x}_{o}}\right)}_{\pi_{l m}\left(\eta_{o}\right)}{ }_{2} \mathrm{Y}_{l m}(\mathbf{e}) .
$$

Sabemos também que harmônicos esféricos de spin podem ser definidos através dos elementos da representação irredutível unitária de $\mathrm{SO}(3)^{1}$ :

$$
\left.D_{m m^{\prime}}^{l}(\alpha, \beta, \gamma)\right|_{\gamma=0}=\sqrt{\frac{4 \pi}{2 l+1}}-m^{\prime} \mathrm{Y}_{l m}^{*}(\beta, \alpha),
$$

Logo,

\footnotetext{
${ }^{1}$ Existe relativa falta de uniformidade na literatura sobre a maneira de relacionar esses objetos. Os harmônicos esféricos de spin surgiram no estudo de representações do grupo BMS (Bondi, Metzner e Sachs [66]) e acabou-se observando que são estreitamente relacionados aos elementos de matriz da representação unitária irredutível do grupo de rotações em três dimensões, podendo mesmo ser definidos em termos destes últimos. Há, entretanto, várias possíveis formas de fazer essa definição e satisfazer os requerimentos necessários, e é este o motivo pelo qual existe falta de uniformidade na literatura.
} 


$$
\frac{Q+i U}{4 I}=\sum_{l m} \pi_{l m 2} \mathrm{Y}_{l m}=\sum_{l m} \pi_{l m}\left(\sqrt{\frac{2 l+1}{4 \pi}} D_{m(-2)}^{l *}\right) \text {. }
$$

Fazemos essa mudança de notação porque sabemos mais fundamentalmente a ação de $\partial$ e $\bar{\partial}$ sobre os elementos das matrizes de Wigner. Posteriormente retornaremos para a notação em termos de harmônicos esféricos de spin, mais comum na literatura sobre CMB.

Com o intuito de escrever (4.16), estudaremos a ação dos operadores $\check{\partial}$ e $\bar{\partial}$ sobre a função de polarização. Para o potencial de lente os efeitos de ðे e $\bar{\partial}$ já foram determinados em (4.7) e (4.8).

$$
\begin{aligned}
\partial P^{++} & =\sum_{l m} \pi_{l m} \partial\left(\sqrt{\frac{2 l+1}{4 \pi}} D_{m(-2)}^{l *}(\mathbf{e})\right) \\
& =\sum_{l m} \sqrt{\frac{2 l+1}{4 \pi}} \pi_{l m} \sqrt{(l-2)(l+3)} D_{m(-3)}^{l *}(\mathbf{e}) .
\end{aligned}
$$

Analogamente,

$$
\overline{\widehat{\partial}} P^{++}=-\sum_{l m} \sqrt{\frac{2 l+1}{4 \pi}} \pi_{l m} \sqrt{(l+2)(l-1)} D_{m(-1)}^{l *}(\mathbf{e}) .
$$

Podemos agora avaliar (4.16). O primeiro termo é:

$$
\begin{aligned}
\bar{\partial} \psi ð P^{++}= & -\sum_{l m} \sum_{l^{\prime} m^{\prime}} \frac{\sqrt{(2 l+1)\left(2 l^{\prime}+1\right)}}{4 \pi} \sqrt{(l-2) l^{\prime}\left(l^{\prime}+1\right)(l+3)} \psi_{l^{\prime} m^{\prime}} \pi_{l m} D_{m^{\prime} 1}^{l^{\prime} *} D_{m(-3)}^{l *} \\
= & -\sum_{l m} \sum_{l^{\prime} m^{\prime}} \underbrace{\frac{1}{4 \pi} \sqrt{(2 l+1)\left(2 l^{\prime}+1\right)(l-2) l^{\prime}\left(l^{\prime}+1\right)(l+3)}}_{A_{l l^{\prime}}} \psi_{l^{\prime} m^{\prime}} \pi_{l m} \\
& \times \sum_{L M M^{\prime}}(-1)^{m+m^{\prime}}\left\langle l^{\prime}-m^{\prime} l-m \mid L M\right\rangle\left\langle L M^{\prime} \mid l^{\prime}-1 l 3\right\rangle D_{M M^{\prime}}^{L} \\
= & -\sum_{L=2}^{\infty} \sum_{M=-L}^{L} \sum_{l=1}^{\infty} \sum_{l^{\prime}=1}^{\infty} \sum_{m=-l}^{l} A_{l l^{\prime}} \psi_{l^{\prime}(-M-m)} \pi_{l m} \\
& \times(-1)^{M}\left\langle l^{\prime}(M+m) l-m \mid L M\right\rangle\left\langle L 2 \mid l^{\prime}-1 l 3\right\rangle D_{M 2}^{L} .
\end{aligned}
$$

Onde usamos regras de composição para os elementos de matrizes de Wigner e propriedades dos coeficientes de Clebsch-Gordan reunidas na Seção D.4 do Apêndice D.

O segundo termo é: 


$$
\begin{aligned}
\partial \psi \overline{\widetilde{\partial}} P^{++}= & -\sum_{L} \sum_{l m} \sum_{l^{\prime} m^{\prime}} \underbrace{\frac{1}{4 \pi} \sqrt{(2 l+1)\left(2 l^{\prime}+1\right)(l-1) l^{\prime}\left(l^{\prime}+1\right)(l+2)}}_{B_{l l^{\prime}}} \psi_{l^{\prime} m^{\prime}} \pi_{l m} \\
& \times \sum_{L M M^{\prime}}(-1)^{m+m^{\prime}}\left\langle l^{\prime}-m^{\prime} l-m \mid L M\right\rangle\left\langle L M^{\prime} \mid l^{\prime} 1 l 1\right\rangle D_{M M^{\prime}}^{L} \\
= & -\sum_{2}^{\infty} \sum_{M=-L}^{L} \sum_{l=1}^{\infty} \sum_{l^{\prime}=1}^{\infty} \sum_{m=-l}^{l} B_{l l^{\prime}} \psi_{l^{\prime}(-M-m)} \pi_{l m} \\
& \times(-1)^{M}\left\langle l^{\prime}(M+m) l-m \mid L M\right\rangle\left\langle L 2 \mid l^{\prime} 1 l 1\right\rangle D_{M 2}^{L} .
\end{aligned}
$$

Portanto,

$$
\begin{aligned}
P^{++}\left(\eta_{o}, \tilde{\mathbf{e}}\right)= & P^{++}\left(\eta_{o}, \mathbf{e}\right)-\frac{1}{2} \sum_{L=2}^{\infty} \sum_{M=-L}^{L}\left\{\sum_{l m} \sum_{l^{\prime}=|l-L|}^{l+L}\left[\psi_{l^{\prime}(-M-m)} \pi_{l m}\right]\left(\eta_{o}\right)(-1)^{M}\left\langle l^{\prime}(M+m) l-m \mid L M\right\rangle\right. \\
& \left.\times\left[A_{l l^{\prime}}\left\langle L 2 \mid l^{\prime}-1 l 3\right\rangle+B_{l l^{\prime}}\left\langle L 2 \mid l^{\prime} 1 l 1\right\rangle\right]\right\} D_{M 2}^{L}(\mathbf{e}) \\
= & P^{++}\left(\eta_{o}, \mathbf{e}\right)-\frac{1}{2} \sum_{L=2}^{\infty} \sum_{M=-L}^{L}\left\{\sum_{l m} \sum_{l^{\prime}=|l-L|}^{l+L}\left[\psi_{l^{\prime}(M-m)} \pi_{l m}\right]\left(\eta_{o}\right)\left\langle l^{\prime}(-M+m) l-m \mid L-M\right\rangle\right. \\
& \left.\times\left[A_{l l^{\prime}}\left\langle L 2 \mid l^{\prime}-1 l 3\right\rangle+B_{l l^{\prime}}\left\langle L 2 \mid l^{\prime} 1 l 1\right\rangle\right]\right\} D_{M-2}^{* L}(\mathbf{e}) \\
= & P^{++}\left(\eta_{o}, \mathbf{e}\right)-\frac{1}{2} \sum_{L=2}^{\infty} \sum_{M=-L}^{L} \Xi_{L M}^{+}\left(\sqrt{\frac{2 L+1}{4 \pi}} D_{M-2}^{* L}(\mathbf{e})\right) \\
= & P^{++}\left(\eta_{o}, \mathbf{e}\right)-\frac{1}{2} \sum_{L=2}^{\infty} \sum_{M=-L}^{L} \Xi_{L M}^{+}\left(\eta_{o}\right)_{2} \mathrm{Y}_{L M}(\mathbf{e})
\end{aligned}
$$

onde definimos os coeficientes $\Xi_{L M}^{+}$como:

$$
\begin{aligned}
\Xi_{L M}^{+}\left(\eta_{o}\right)= & \sum_{l m} \sum_{l^{\prime}=|l-L|}^{l+L} \sqrt{\frac{4 \pi}{2 L+1}}\left[\psi_{l^{\prime}(M-m)} \pi_{l m}\right]\left(\eta_{o}\right)\left\langle l^{\prime}(-M+m) l-m \mid L-M\right\rangle \\
& \times\left[A_{l l^{\prime}}\left\langle L 2 \mid l^{\prime}-1 l 3\right\rangle+B_{l l^{\prime}}\left\langle L 2 \mid l^{\prime} 1 l 1\right\rangle\right] .
\end{aligned}
$$

Em breve esclareceremos o significado de $\left[\psi_{l^{\prime}(M-m)} \pi_{l m}\right]\left(\eta_{o}\right)$.

Finalmente escrevemos: 


$$
P^{++}\left(\eta_{o}, \tilde{\mathbf{e}}\right)=\sum_{L=2}^{\infty} \sum_{M=-L}^{L}\left(\pi_{L M}\left(\eta_{o}\right)-\frac{1}{2} \Xi_{L M}^{+}\left(\eta_{o}\right)\right){ }_{2} \mathrm{Y}_{L M}(\mathbf{e}) .
$$

Vemos que, de modo semelhante ao que acontece para a temperatura, esta expressão permite calcular a nova polarização não apenas como uma correção à antiga, mas como uma correção a seus coeficientes em uma expansão nos harmônicos esféricos de spin.

Se escrevêssemos - de acordo com (D.10):

$$
P^{--}\left(\eta_{o}, \tilde{\mathbf{e}}\right):=\frac{Q-i U}{4 I}\left(\eta_{o}, \tilde{\mathbf{e}}\right),
$$

através da mesma sequência de etapas poderíamos mostrar que ${ }^{2}$ :

$$
P^{--}\left(\eta_{o}, \tilde{\mathbf{e}}\right)=\sum_{L=2}^{\infty} \sum_{M=-L}^{L}\left(\pi_{L M}\left(\eta_{o}\right)-\frac{1}{2} \Xi_{L M}^{-}\left(\eta_{o}\right)\right){ }_{-2} \mathrm{Y}_{L M}(\mathbf{e})
$$

onde

$$
\begin{aligned}
\Xi_{L M}^{-}\left(\eta_{o}\right)= & \sum_{l m} \sum_{l^{\prime}=|l-L|}^{l+L} \sqrt{\frac{4 \pi}{2 L+1}}\left[\psi_{l^{\prime}(M-m)} \pi_{l m}\right]\left(\eta_{o}\right)\left\langle l^{\prime}(-M+m) l-m \mid L-M\right\rangle \\
& \times(-1)^{l+l^{\prime}-L}\left[A_{l l^{\prime}}\left\langle L 2 \mid l^{\prime}-1 l 3\right\rangle+B_{l l^{\prime}}\left\langle L 2 \mid l^{\prime} 1 l 1\right\rangle\right] .
\end{aligned}
$$

Vemos neste ponto, comparando (4.24) e (4.27) que $\Xi_{L M}^{+} \neq \Xi_{L M}^{-}$sempre que $l+l^{\prime}-L$ não for par na soma (4.27) e, portanto, além do modo $E$ preexistente o efeito de lentes gravitacionais é capaz de induzir um modo $B$ de polarização. Este modo $B$ naturalmente não é oriundo de mecanismos relacionados à dinâmica dos espalhamentos na recombinação mas sim originário da deformação do modo $E$ preexistente pelo efeito dos campos gravitacionais.

\subsection{O efeito do potencial de lente}

Notamos observando os desenvolvimentos anteriores que tanto os coeficientes $\theta_{l m}, \pi_{l m}$ quanto $\psi_{l m}$ que aparecem nas expressões da temperatura e polarização são dados em termos de integrais sobre o tempo conforme em intervalos definidos. Como sabemos que os efeitos de lente devem ser acumulados

\footnotetext{
${ }^{2} \mathrm{O}$ fato de os coeficientes na expansão de $P^{--}$serem os mesmos de $P^{++}$vem do fato bem conhecido de ser nulo o modo $B$ da polarização gerada através de espalhamentos Thomson entre elétrons e fótons. Como aqui não temos a intenção de apresentar a discussão em termos de modos $E$ e $B$, não demonstraremos este fato. Como referência para tal demonstração, sugerimos [50] ou [52].
} 
102 Efeito de lentes fracas sobre a temperatura e polarização da CMB: estudo no espaço das posições apenas enquanto o fóton propaga-se livremente, podemos agrupar os domínios de integração esclarecendo o que pretendíamos denotar por $\left[\psi_{l^{\prime}(M-m)} \theta_{l m}\right]\left(\eta_{o}\right)$ e $\left[\psi_{l^{\prime}(M-m)} \pi_{l m}\right]\left(\eta_{o}\right)$. Para tanto, lembrêmo-nos que, por (2.71), o potencial de lente escreve-se como:

$$
\begin{aligned}
\psi\left[\left(\eta_{o}-\eta\right),\left(\eta_{o}-\eta\right) \mathbf{e}\right]= & -2 \int_{\eta_{o}-\eta}^{\eta_{o}} \frac{\left(\eta^{\prime}-\eta\right)}{\left(\eta_{o}-\eta\right)\left(\eta_{o}-\eta^{\prime}\right)} \\
& \times \Psi_{W}\left[\left(\eta_{o}-\eta^{\prime}\right),\left(\eta_{o}-\eta^{\prime}\right) \mathbf{e}\right] d \eta^{\prime} .
\end{aligned}
$$

Os coeficientes $\Xi_{l m}\left(\eta_{o}\right)$ definidos em (4.12) são dados em termos do produto $\left[\psi_{l^{\prime}(M-m)}\left(\eta_{o}-\eta\right) \theta_{l m}\right]\left(\eta_{o}\right)$, e os $\Xi_{l m}^{+}\left(\eta_{o}\right)$ definidos em (4.24) são dados em termos do produto $\left[\psi_{l^{\prime}(M-m)}\left(\eta_{o}-\eta\right) \pi_{l m}\right]\left(\eta_{o}\right)$ (e de modo análogo $\Xi_{l m}^{-}$). Para o cálculo dos coeficientes do potencial de lente deve-se calcular toda a influência gravitacional - balanceada por distâncias - ao longo da trajetória desde o momento em que está livre para propagar-se, ou seja, desde o momento em que se desacopla. Podemos, portanto, incluir a integração que toma conta dos efeitos do potencial de lente entre as integrais que nos permitem calcular os coeficientes de expansão para a temperatura ou a polarização. Tomando, por exemplo, o caso particular das equações de Boltzmann em que se desacopla a evolução da temperatura, sabe-se que os coeficientes de expansão da polarização podem ser escritos como (reescrevendo (3.22)):

$$
\begin{aligned}
\pi_{l m}\left(\eta_{o}\right) & =-\frac{3}{4 \pi} \sqrt{\frac{(l+2) !}{(l-2) !}} \int_{0}^{\eta_{o}} d \eta_{f} \mu^{\prime}\left(\eta_{f}\right) \mathrm{e}^{-\mu\left(\eta_{f}\right)} \\
& \times \sum_{n=1}^{\infty} \frac{1}{(n-1) !} \int_{0}^{\eta_{f}} d \eta^{\prime} \ldots d \eta_{i} \mathrm{~T}\left\{\mu^{\prime}\left(\eta^{\prime}\right) \mathrm{e}^{-\mu\left(\eta^{\prime}\right)} \ldots \mu^{\prime}\left(\eta_{i}\right) \mathrm{e}^{-\mu\left(\eta_{i}\right)}\right\} \\
& \times \frac{9^{(n-1)}\left(\frac{\pi}{2}\right)^{(n+1) / 2}}{(\Gamma(7 / 2))^{n-1}} \int_{0}^{\eta_{i}} d \eta \int_{0}^{\infty} d x x^{2} S_{l m}(x, \eta) \\
& \times \int d(\cos \alpha)\left(\frac{X \Delta \eta_{n}}{r^{3}}\right)^{2} P_{l}^{-2}(\cos \alpha) \operatorname{sen}^{2} \alpha p_{n}\left(r ; \Delta \eta_{1}, \ldots, \Delta \eta_{n} \mid 7\right) .
\end{aligned}
$$

Podemos incluir o efeito do potencial de lente para calcular os coeficientes $\left[\psi_{l^{\prime}(M-m)} \pi_{l m}\right]\left(\eta_{o}\right)$ da seguinte maneira: 


$$
\begin{aligned}
{\left[\psi_{l^{\prime}(M-m)} \pi_{l m}\right]\left(\eta_{o}\right) } & =\frac{3}{2 \pi} \sqrt{\frac{(l+2) !}{(l-2) !}} \int_{0}^{\eta_{o}} d \eta_{f} \mu^{\prime}\left(\eta_{f}\right) \mathrm{e}^{-\mu\left(\eta_{f}\right)} \\
& \times \int_{\eta_{o}-\eta_{f}}^{\eta_{o}} \frac{\left(\eta_{\ell}-\eta_{f}\right)}{\left(\eta_{o}-\eta_{f}\right)\left(\eta_{o}-\eta_{\ell}\right)}\left[\left(\Psi_{W}\right)_{l^{\prime}(M-m)}\right]\left(\eta_{o}-\eta_{\ell}\right) d \eta_{\ell} \\
& \times \sum_{n=1}^{\infty} \frac{1}{(n-1) !} \int_{0}^{\eta_{f}} d \eta^{\prime} \ldots d \eta_{i} \mathrm{~T}\left\{\mu^{\prime}\left(\eta^{\prime}\right) \mathrm{e}^{-\mu\left(\eta^{\prime}\right)} \ldots \mu^{\prime}\left(\eta_{i}\right) \mathrm{e}^{-\mu\left(\eta_{i}\right)}\right\} \\
& \times \frac{9^{(n-1)}\left(\frac{\pi}{2}\right)^{(n+1) / 2}}{(\Gamma(7 / 2))^{n-1}} \int_{0}^{\eta_{i}} d \eta \int_{0}^{\infty} d x x^{2} S_{l m}(x, \eta) \\
& \times \int d(\cos \alpha)\left(\frac{x \Delta \eta_{n}}{r^{3}}\right)^{2} P_{l}^{-2}(\cos \alpha) \operatorname{sen}^{2} \alpha \\
& \times p_{n}\left(r ; \Delta \eta_{1}, \ldots, \Delta \eta_{n} \mid 7\right)
\end{aligned}
$$

ou seja, apenas inclui-se a integral que descreve o potencial de lente (Eq. (2.66)) como um integrando extra na integral de propagação livre após todos os espalhamentos que geraram sinal em (3.22). Se fôssemos representar diagramaticamente a Eq. (4.30), escreveríamos:

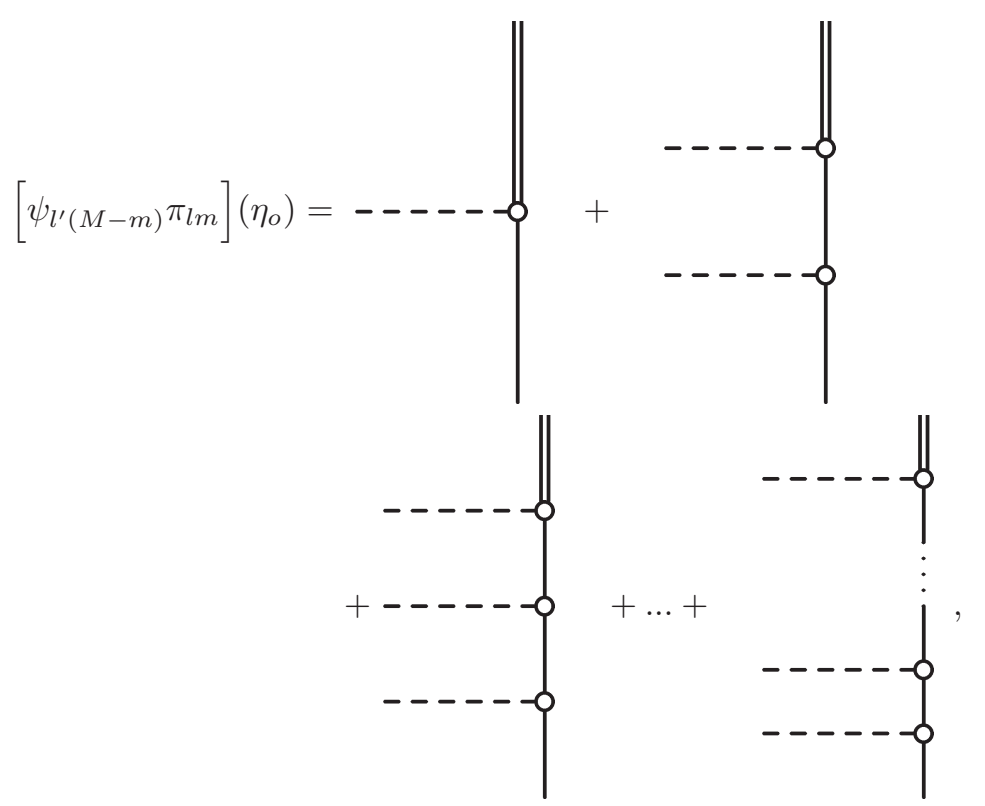

onde representamos por linhas contínuas duplas o trecho sobre o qual o potencial de lentes intervém.

Poderíamos, por exemplo, estar interessados no efeito de lentes sobre as 
104 Efeito de lentes fracas sobre a temperatura e polarização da CMB: estudo no espaço das posições correções em segunda ordem na polarização calculado em (3.34). Neste caso, temos:

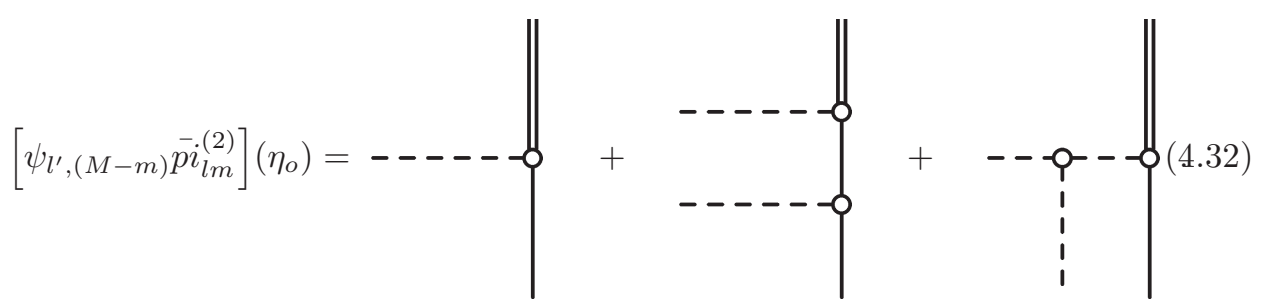

Da mesma maneira, se quiséssemos calcular o efeito de lentes sobre correções em segunda ordem sobre a temperatura calculado em (3.30), teríamos:

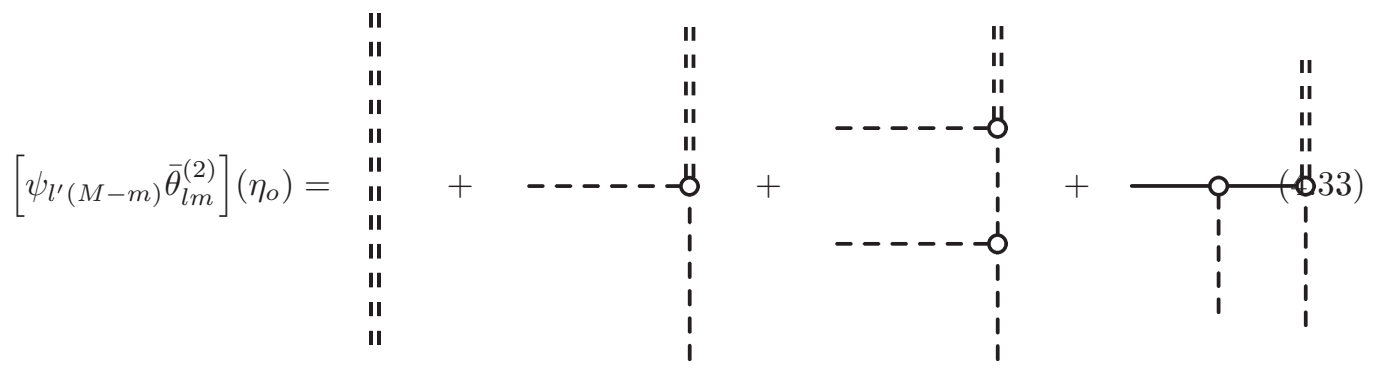

As linhas pontilhadas duplas indicam o trecho sobre o qual o potencial de lentes contribui no cálculo dos coeficientes de expansão da temperatura.

\subsection{Resumo}

Calculando

$$
\begin{aligned}
\Xi_{L M}\left(\eta_{o}\right)= & \sum_{l m} \sum_{l^{\prime}=|l-L|}^{l+L} \sqrt{\frac{4 \pi}{2 L+1}}\left[\psi_{l^{\prime}(M-m)} \theta_{l m}\right]\left(\eta_{o}\right)\left\langle l^{\prime}(-M+m) l-m \mid L-M\right\rangle \\
& \times Z_{l l^{\prime}}\left\langle L 0 \mid l^{\prime}-1 l 1\right\rangle\left[1+(-1)^{l+l^{\prime}-L}\right], \\
\Xi_{L M}^{+}\left(\eta_{o}\right)= & \sum_{l m} \sum_{l^{\prime}=|l-L|}^{l+L} \sqrt{\frac{4 \pi}{2 L+1}}\left[\psi_{l^{\prime}(M-m)} \pi_{l m}\right]\left(\eta_{o}\right)\left\langle l^{\prime}(-M+m) l-m \mid L-M\right\rangle \\
& \times\left[A_{l l^{\prime}}\left\langle L 2 \mid l^{\prime}-1 l 3\right\rangle+B_{l l^{\prime}}\left\langle L 2 \mid l^{\prime} 1 l 1\right\rangle\right]
\end{aligned}
$$

e 


$$
\begin{aligned}
\Xi_{L M}^{-}\left(\eta_{o}\right)= & \sum_{l m} \sum_{l^{\prime}=|l-L|}^{l+L} \sqrt{\frac{4 \pi}{2 L+1}}\left[\psi_{l^{\prime}(M-m)} \pi_{l m}\right]\left(\eta_{o}\right)\left\langle l^{\prime}(-M+m) l-m \mid L-M\right\rangle \\
& \times(-1)^{l+l^{\prime}-L}\left[A_{l l^{\prime}}\left\langle L 2 \mid l^{\prime}-1 l 3\right\rangle+B_{l l^{\prime}}\left\langle L 2 \mid l^{\prime} 1 l 1\right\rangle\right],
\end{aligned}
$$

$\operatorname{com}\left[\psi_{l^{\prime}(M-m)} \theta_{l m}\right]\left(\eta_{o}\right)$ e $\left[\psi_{l^{\prime}(M-m)} \pi_{l m}\right]\left(\eta_{o}\right)$ dados pelos diagramas com linhas duplas, obteremos as correções para a temperatura e polarização devido ao efeito de lentes gravitacionais. As expressões para a temperatura e a polarização são:

$$
\begin{aligned}
& \Theta\left(\eta_{o}, \tilde{\mathbf{e}}\right)=\sum_{L=0}^{\infty} \sum_{M=-L}^{L}\left(\theta_{L M}\left(\eta_{o}\right)-\frac{1}{2} \Xi_{L M}\left(\eta_{o}\right)\right) \mathrm{Y}_{L M}(\mathbf{e}) \\
& P^{++}(\tilde{\mathbf{e}})=\sum_{L=2}^{\infty} \sum_{M=-L}^{L}\left(\pi_{L M}\left(\eta_{o}\right)-\frac{1}{2} \Xi_{L M}^{+}\left(\eta_{o}\right)\right){ }_{2} \mathrm{Y}_{L M}(\mathbf{e})
\end{aligned}
$$

e

$$
P^{--}(\tilde{\mathbf{e}})=\sum_{L=2}^{\infty} \sum_{M=-L}^{L}\left(\pi_{L M}\left(\eta_{o}\right)-\frac{1}{2} \Xi_{L M}^{-}\left(\eta_{o}\right)\right){ }_{-2} \mathrm{Y}_{L M}(\mathbf{e})
$$

Essas equações encapsulam os procedimentos de cálculo das fontes da polarização (termo que envolve $S_{l m}$ ) e incorporam o efeito de lente causado pelos potenciais de Bardeen durante a fase de propagação livre dos fótons, sendo o momento de desacoplamento $\left(\eta_{f}\right)$ determinado probabilisticamente através da função de visibilidade. O efeito de lente sobre a temperatura e polarização será incluído via integração mediada pelo potencial de Weyl do sinal de temperatura ou polarização que se teria sem o efeito de lentes. A integração que inclui o potencial de Weyl faz-se apenas no trecho de livre propagação do fóton, ou seja, após seu último espalhamento ocorrido durante a recombinação. 


\section{Discussão}

Este trabalho concentrou-se essencialmente em três problemas: o estudo de lentes gravitacionais, a descrição da hierarquia de Boltzmann para a CMB no espaço das posições e o estudo de lentes gravitacionais sobre a CMB.

Tratanto o problema das lentes gravitacionais, decidimos não nos restringir apenas aos elementos que seriam necessários para o estudo específico de lentes sobre a radiação cósmica de fundo. Para além disso, decidimos percorrer tópicos tais como determinação de cenários com imagens múltiplas, que não aparenta ser diretamente associado ao problema de lentes sobre a CMB.

Fizemos isso porque se há diferença na fenomenologia dos problemas, há proximidade na estrutura matemática que os descreve: são ambos manifestações do mesmo problema variacional. Os campos de Jacobi que descrevem o afastamento de geodésicas vizinhas são também responsáveis por determinar pontos a partir dos quais extremos de um funcional perdem suas propriedades minimizantes. Considerando-se a segunda variação de energia no problema variacional define-se uma forma bilinear cujo índice determina o número de pontos conjugados ao longo de uma geodésica. Este número, por sua vez, é relacionado através de um conjunto de desigualdades associadas à natureza topológica do espaço de curvas variacionais associadas à uma curva inicial de interesse. Assim, as manifestações físicas bastante diferentes no caso de uma lente forte (que dá origem a imagens múltiplas) e de uma lente fraca são expressões de um mesmo problema em que pontos críticos estão ou não presentes. O estudo dos campos de Jacobi é então central na formulação do problema das lentes gravitacionais.

Se os campos de Jacobi são centrais na descrição da natureza dos extremos do problema variacional por um lado, eles estão também profundamente associados à geometria dos espaços-tempo, já que são determinados por uma equação diferencial de segunda ordem onde comparece a curvatura de Riemann. A relação direta entre os campos de Jacobi com objeto tão central na geometria das variedades (e não com contrações da curvatura, por exemplo) insere também os campos de Jacobi na categoria de objetos centrais na teoria 
da relatividade geral. No estudo de teoremas de singularidade, por exemplo, a existência de pontos conjugados ao longo de geodésicas é ponto fundamental no argumento das demonstrações. Aprendemos também dessa relação que para uma ampla classe de espaços-tempo, lentes gravitacionais sempre podem dar origem a imagens múltiplas contanto que se as prolongue suficientemente.

Os problemas variacionais, no entanto, não se encerram no tratamento apenas da equação de Jacobi e de pontos conjugados. Com efeito, a equação de Jacobi fornece uma aproximação linear para o problema do afastamento de geodésicas vizinhas. Correções de segunda ordem, por exemplo, poderiam ser incluídas [67] mas não encerrariam a discussão: fenômenos críticos estão também presentes se geodésicas não vizinhas voltam a cruzar-se e a discussão sobre as condições necessárias para a existência de imagens múltiplas envolve o conceito de ponto de corte. Tratar um problema que se assume ser de lentes fracas requer, portanto, entender se métodos de aproximação lineares são suficientes, ou seja, é necessário conhecer as condições em que pontos de corte surgem. Um exemplo bastante crítico é o da corda cósmica quando tomamos um tensor de energia-momento na forma de uma distribuição. Neste caso a curvatura do espaço é nula, não há pontos conjugados ao longo de nenhuma geodésica nula e no entanto há imagens múltiplas pois há pontos de corte.

Os campos de Jacobi estão presentes também no estudo de outros aspectos do problema da propagação de geodésicas nulas em espaços-tempo. Na determinação de geodésicas nulas em um espaço-tempo perturbado à partir das geodésicas nulas do espaço-tempo base, por exemplo, o afastamento entre as geodésicas em ambos os espaços é determinado pela solução de uma equação de Jacobi não homogênea. A evolução da forma das seções transversais de um feixe de geodésicas emergindo de uma fonte pontual também pode ser descrita através do conhecimento dos campos de Jacobi. Com efeito, exploramos a maneira (única) de escrever estes campos de Jacobi através da aplicação exponencial para derivar a aplicação de Jacobi relacionando as projeções da velocidade de afastamento de geodésicas na origem com o afastamento entre elas em outro valor do parâmetro afim. Como buscamos afastamento relativo de geodésicas nulas, apenas componentes em direções tipo-espaço interessam e isso nos levou a considerar classes de Jacobi. Utilizando propriedades da aplicação exponencial e o fato de que o conjunto de pontos conjugados ao longo de uma geodésica tem medida nula, construímos explicitamente um ten- 
108 Discussão

sor de Jacobi, que mostramos ser exatamente o objeto que realiza o que se ambicionava derivar em [1], mas cuja argumentação apresentava problemas. Nossa derivação deu origem a [5]. Resolvemos as equações obtidas no caso de um espaço-tempo de Friedmann-Lamaître-Robertson-Walker sujeito a perturbações escalares para obter um mapa de lente no contexto da cosmologia. Isso se pode fazer graças à simplicidade dos tensores de Ricci e Weyl no espaçotempo em questão e também porque pudemos calcular as contribuições desses objetos sobre geodésicas nulas do espaço-tempo base, ou seja, usamos a aproximação de Born. A justificativa da aproximação de Born foi nossa despedida das aplicações de campos de Jacobi neste trabalho.

Passamos então ao problema da radiação cósmica de fundo propriamente dito. Tomando a hierarquia de Boltzmann que descreve temperatura e polarização, mostramos como é possível estabelecer um tratamento no espaço das posições. Este resultado havia sido iniciado em [4] e foi apresentado de maneira mais completa em [3]. Desenvolvemos um formalismo de certa maneira aproximado ao tratamento via integração funcional de processos de Poisson [68]. Mostramos, desacoplando a evolução de partes da hierarquia, que densidades de probabilidade associadas a vôos aleatórios surgiam diretamente da estrutura equações quando tratadas no espaço das posições, semelhantemente ao que acontece no tratamento da equação de telegrafista como um processo estocástico [59, 68]. Com efeito, como comum a equações hiperbólicas, mostramos que relações causais são satisfeitas na hierarquia de Boltzmann considerada. Resta, no entanto, a questão de quais as características da hierarquia que permitiram esse tipo de tratamento e quais as categorias de equações de Boltzmann que se permitem tratar da mesma maneira.

O tipo de tratamento introduzido também nos permitiu formalizar uma teoria de perturbação em termos do número de espalhamentos sofridos pelos fótons durante a recombinação. Além de fatores relativos às seções de choque de todos os possíveis espalhamentos, o número de espalhamentos comparece nas densidades de probabilidades de vôos aleatórios que atribuem peso às possíveis diferentes histórias dos fótons durante a recombinação. Essa estrutura de expansão nos permitiu checar (recorrendo a algumas aproximações para poder usar um resultado assintótico) que, independentemente da supressão advinda das seções de choque, termos relativos a um grande número de espalhamentos implementam correções desprezíveis aos observáveis. Podemos interpretar 
este fato como uma ilustração da afirmação do teorema $H$ de Boltzmann, que diz que o aumento do número de interações aproxima o sistema do estado de equilíbrio.

Embora tenha propriedades formais interessantes e permita uma interpretação à la teoria cinética da física da radiação cósmica de fundo, o formalismo apresentado por nós não poderia diferir mais do que o que se encontra em geral na literatura (que pode ser representada por [49]). Uma vantagem desse afastamento vem da diferença nos mecanismos de interromper as iterações das equações. Para nós o limite de precisão é dado por um número máximo de espalhamentos considerados durante a recombinação. Nos tratamentos usuais a precisão é determinada pelo número de multipolos utilizados para a evolução do sistema. Resta-nos ainda compreender a correspondência entre as duas linguagens.

Tendo desenvolvido um formalismo para o tratamento da temperatura e polarização da radiação cósmica de fundo no espaço das posições e também a estrutura do mapa de lente em espaços de FLRW, pudemos iniciar o estudo do problema de lentes gravitacionais sobre a radição de fundo, que era nosso objetivo inicial. Relacionando operadores de derivada covariante sobre a esfera com operadores diferenciais que atuam sobre os elementos da representação unitária irredutível do grupo de rotações em três dimensões, pudemos calcular o efeito das lentes gravitacionais sobre a parte angular das decomposições dos campos de temperatura e polarização. Especificamente, mostramos que o efeito de lentes pode ser introduzido por uma correção aos coeficientes de expansão obtidos no caso sem lentes. Indicamos que modos de polarização com duas diferentes paridades são presentes quando há o efeito de lentes, o que mostra de que modo $B$ é gerado a partir do modo $E$ introduzido pelos espalhamentos Thomson durante a recombinação, muito embora tenhamos decidido não discutir o problema da polarização em termos desses modos. Mostramos também que a parte radial das expansões dos campos são modificadas pela presença dos potenciais gravitacionais. Aproveitando a descrição da radiação cósmica de fundo no espaço das posições, pudemos inserir o efeito do potencial de lente diretamente sobre a fase de propagação livre dos fótons expressa entre as integrais que determinam os coeficientes de expansão da temperatura e polarização. Resta-nos ainda, no entanto, muito a estudar sobre este assunto. Interessa-nos, por exemplo, investigar mecanismos de de-lensing nesse formalismo. 


\section{Discussão}

Em tempo, estudando as integrais presentes no problema de vôos aleatórios e tentando fazer a conexão com as integrais que emergiam da hierarquia de Boltzmann em nosso problema, pudemos formular o conceito de vôo aleatório estendido apresentado em [3] e [6]. Estudando expansões de Fourier-Bessel aplicadas a esses vôos aleatórios estendidos fomos capazes de demonstrar uma identidade semelhante à relação de completeza para funções de Bessel esféricas no intervalo $[0,1]$ que havia sido proposta em [4] mas que não havíamos podido demonstrar naquele momento. A demonstração é apresentada no Apêndice C.4 e deu origem a [6]. 


\section{Apêndice A Teoremário}

As derivações apresentadas nos primeiros capítulos do texto utilizam frequentemente propriedades de elementos típicos da geometria ou da relatividade geral cujas definições e propriedades básicas escolhidas são apresentadas aqui por conveniência. Serão nossos temas aqui estrutura causal, geometria Lorentziana, conexões, curvaturas, geodésicas (e o mapa exponencial) e campos de Jacobi.

\section{A.1 Geometria de vetores nulos}

Como os raios de luz correspondem a geodésicas nulas em um dado espaçotempo, convém listar uma coleção de definições e fatos básicos sobre vetores nulos em geometria Lorentziana. (Essa seção tem como referências básicas [69] e [70]).

Definição A.1 Seja $V$ um espaço-tempo e $W \subset V$ um subespaço. $O$ caráter causal de $W$ é

- tipo-espaço se, e somente se, g é positiva definida em W.

- tipo luz ou nulo se e somente se g é positiva semi-definida mas não positiva definida em $W$.

- tipo-tempo caso o contrário.

Suponha que $v \in V$. O caráter causal de $v$ é aquele do $\operatorname{span}(v)$. $v$ é definido como causal se e somente se $v$ não é tipo-espaço.

Observações:

- $\mathrm{O}$ vetor zero é declarado tipo-espaço ${ }^{1}$. Um vetor $v \in V$ é tipo-espaço se e somente se $\langle v, v\rangle>0$, nulo se e somente se $\langle v, v\rangle=0$ e tipo-tempo se e somente se $\langle v, v\rangle<0$.

\footnotetext{
${ }^{1}$ Não há critério universalmente aceito sobre o caráter do vetor zero.
} 
112 Teoremário

- Um subespaço $W \subset V$ é tipo-espaço se e somente se todos seus vetores são tipo-espaço, nulo se ele contém um vetor nulo mas nenhum vetor tipotempo e tipo-tempo se contém um vetor tipo-tempo.

- Nenhum dos casos acima são vazios.

Definição A.2 Seja $(V,\langle.,\rangle$.$) um espaço vetorial dotado de um produto escalar$ $\langle.,$.$\rangle . Se v, w \in V$, dizemos que $v$ é ortogonal $a w, v \perp w$, se $\langle v, w\rangle=0$. Consequentemente, para $A, B \subseteq V$, dizemos que $A$ é ortogonal $a B, A \perp B$, se $v \perp w$ para todo $v \in A$ e $w \in B$. Denotaremos

$$
A^{\perp}=\{w \in V:\langle v, w\rangle=0, \forall v \in A\}
$$

Além disso, uma base $e_{1}, e_{2}, \ldots, e_{n}$ de $V$ é dita ortonormal se seus elementos são unitários, isto é, $\left|e_{i}\right|=\sqrt{\left|\left\langle e_{i}, e_{i}\right\rangle\right|}=1, i=1, \ldots, n$ e ortogonais uns aos outros, $\left\langle e_{i}, e_{j}\right\rangle=0, i, j=1, \ldots, n$.

Lema A.3 Dada uma base ortonormal $B$ de $(V,\langle.,\rangle$.$) , o número \nu$ de vetores tipo-tempo na base não depende da escolha da base, mas apenas de $(V,\langle.,\rangle$.

Por completeza e por ter a disposição os ingredientes,

Definição A.4 Dizemos que o produto escalar $\langle.,$.$\rangle é Lorentziano se \nu=1$ e $n \geq 2$.

Definição A.5 Seja $(V,\langle.,\rangle$.$) um espaço dotado de um produto escalar. Um$ subespaço $W \subset V$ é dito não-degenerado se $W \cap W^{\perp}=\{0\}$ (ou, equivalentemente, se a restrição da métrica ao subespaço é não-degenerada).

Proposição A.6 Seja $W \subset V$ não degenerado. Então,

- $\operatorname{dim} W+\operatorname{dim} W^{\perp}=\operatorname{dim} V$;

- $W^{\perp \perp}=W$;

- $V=W \oplus W^{\perp} \Longleftrightarrow W$ é não degenerado.

Com o uso dessa proposição pode-se mostrar a validade da seguinte outra proposição que caracteriza as estruturas causais de subespaços e de seus complementos. 
Proposição A.7 Seja $W \subset V$ um subespaço. (a) $W$ é tipo-tempo $\Longleftrightarrow W^{\perp}$ é tipo-espaço e $W$ é tipo-espaço $\Longleftrightarrow W^{\perp}$ é tipo-tempo. (b) $W$ é nulo $\Longleftrightarrow$ $W \cap W^{\perp} \neq\{0\} \Longleftrightarrow W^{\perp}$ é nulo.

Seguem dois corolários:

Corolário A.8 $w \in W$ é tipo-tempo se e somente se $w^{\perp} \subset V$ é tipo-espaço.

Corolário A.9 Dois vetores nulos são ortogonais se e somente se eles são proporcionais.

\section{Demonstração}

Sejam $v, w \in V$ nulos, $e \in V$ tipo-tempo e $\langle v, w\rangle=0$. Então, pelo Corolário A. $8\langle e, v\rangle \neq 0$. Logo, para algum $a \in \mathbb{R},\langle e, w+a v\rangle=0$. Então $w+a v$ é tipo-espaço, novamente pelo Corolário A.8. Mas $\langle w+a v, w+a v\rangle=\langle w, w\rangle+$ $2 a\langle v, w\rangle+\langle v, v\rangle=0$ donde segue que $w+a v=0$ e os vetores são proporcionais. O inverso é trivial.

A seguinte proposição também é importante para as construções apresentadas no texto:

Proposição A.10 Se $W \subset V$, com $\operatorname{dim}(W) \geq 2$, as seguintes condições são equivalentes:

(i) $W$ é tipo-tempo,

(ii) W contém dois vetores nulos linearmente independentes,

(iii) $W$ contém um vetor tipo-tempo.

\section{A.2 Estrutura causal}

As referências principais para esta seção são [15] e [70].

Com o intuito de fixar nomenclatura e notação apresentaremos as seguintes definições:

Definição A.11 Seja $(M, g)$ um espaço-tempo e tomemos $p, q \in M$. Diremos que:

- $p$ está no passado cronológico de $q$, denotado por $p \ll q$, se existe uma curva tipo-tempo suave por partes e direcionada para o futuro que parte de p e chega a q; 
114 Teoremário

- $p$ está no passado estritamente causal de $q$, denotado por $p<q$, se existe uma curva causal suave por partes e direcionada para o futuro que parte de $p$ e chega a q;

- $p$ está no passado causal de $q$, denotado por $p \leq q$, se $p \ll q$ ou $p<q$.

Dualmente definem-se os futuros cronológico, estritamente causal e causal de um par de pontos de $M$. Estas definições estendem-se naturalmente a subconjuntos $A \subset M$ e normalmente são referenciados como conjuntos:

$$
I^{-}(A)=\{q \in M \mid \exists p \in A \text { tal que } q \ll p\}
$$

é normalmente chamado de passado cronológico de $A$ e

$$
J^{-}(A)=\{q \in M \mid \exists p \in A \text { tal que } q \leq p\}
$$

é normalmente chamado de passado causal de $A$. Os futuros cronológico e causal de $A$ são dualmente definidos e denotados por $I^{+}(A)$ e $J^{+}(A)$, respectivamente.

Consideremos ainda a seguinte definição, estabelecendo tipos de estrutura causal:

Definição A.12 Seja $(M, g)$ um espaço-tempo. Diremos que $(M, g)$ é

(i) CRONOLÓGICO se não existem curvas tipo-tempo fechadas em $M$;

(ii) CAUSAL se não existem curvas causais fechadas em $M$;

(iii) Distinguível se cada ponto é caracterizado por seu futuro cronológico e também por seu passado cronológico, isto é, se $p, q \in M$ satisfazem $I^{+}(p)=I^{+}(q)$ e $I^{-}(p)=I^{-}(q)$ então $p=q ;$

(iv) FORTEMENTE CAUSAL se dada uma vizinhança $U \ni p$ existe uma vizinhança $V \subset U, p \in V$, tal que qualquer curva com pontos finais em $V$ está inteiramente contida em $V$;

(v) ESTAVELMENTE CAUSAL se o espaço-tempo admite uma função tempo, isto é, uma função contínua que é estritamente crescente ao longo de qualquer curva causal direcionada para o futuro;

(vi) CAUSALMENTE SIMPLES se é causal e os subconjuntos $J^{+}(p)$ e $J^{-}(p)$ são fechados em $M$, para todo $p \in M$;

(vii) GLOBALMENTE HIPERBÓLICO se é causal e cada intersecção $J^{+}(p) \cap J^{-}(q)$ é um subconjunto compacto de $M$, para todo $p, q \in M$. 
Pode-se demonstrar [70] que os ítens mais abaixo na lista tem todas as propriedades listadas mais acima dele, ou seja, um espaço que seja causal é também cronológico, um espaço distinguível é causal e cronológico e assim sucessivamente.

Convém refrasear algumas das definições de modo que se tornem mais intuitivas ou detalhadas, especialmente os mais utilizados em nossos problemas. Por exemplo, um espaço-tempo é distinguível se e somente se para qualquer vizinhança $U \ni p$ existe uma vizinhança $V \subset U, p \in V$ tal que qualquer curva causal que se inicia em $p$ e deixa $V$ não pode reentrar em $V$. Espaços-tempo fortemente causais não admitem curvas "praticamente fechadas", ou seja, $(M, g)$ não é fortemente causal em um ponto $p$ de $M$ se existe uma curva causal com pontos finais arbitrariamente próximos de $p$ (mas deixando uma vizinhança fixa de $p$ ). Uma condição necessária para que um espaço-tempo seja globalmente hiperbólico, é que seja simplesmente causal e que não contenha singularidades nuas ${ }^{2}$.

Uma característica também central em espaços globalmente hiperbólicos e que será várias vezes empregada ou suposta é que eles admitem uma função tempo sobrejetora $t: M \rightarrow \mathbb{R}$ tal que suas superfícies de nível $S_{t_{0}}=t^{-1}\left(t_{0}\right)$, $t_{0} \in \mathbb{R}$, são hipersuperfícies de Cauchy ${ }^{3}$ tipo-espaço e por esta razão o espaçotempo é isométrico a um produto

$$
S \times \mathbb{R} \quad g=-\beta d t^{2}+\bar{g},
$$

com $S=t^{-1}(0), \beta: M \rightarrow \mathbb{R}$ uma função suave positiva e $\bar{g}$ uma métrica riemanniana (dependente de $t_{0}$ ) restrita às hipersuperfícies de Cauchy $S_{t_{0}}{ }^{4}$.

\footnotetext{
${ }^{2}$ Porque a não compacidade de $J^{+}(p) \cap J^{-}(q)$ permite a construção de certas curvas que se iniciam em $p$, podem ser sempre observadas em $q$ mas que "desaparecem" repentinamente do espaço-tempo, ou seja, encontram algum tipo de singularidade. O fato da singularidade ser nua significa que ela ou pelo menos o desaparecimento de curvas (ou partículas) - pode ser observada por $q$. [70]

${ }^{3}$ Uma hipersuperfície de Cauchy é um subconjunto $S$ de $M$ que é atravessado apenas uma vez por qualquer curva tipo-tempo inextensível.

${ }^{4}$ Uma lacuna clássica em relatividade geral centrava-se em torno desta decomposição e apenas recentemente foi resolvida. [71] mostra haver uma tal função $\beta$ contínua. [15] afirma sem demonstrar (em sua proposição 6.6.8) a possibilidade de suavizar a função obtida por Geroch. A demonstração final do fato, entretanto, é recente e apresentada em [72] e [73]. Uma revisão sobre os estudos sobre esse tema podem ser encontrada em [74].
} 
116 Teoremário

\section{A.3 Conexões}

Seja $\mathfrak{X}(M)$ o conjunto dos campos vetoriais diferenciáveis em $M$. Seguindo [17], definimos:

Definição A.13 Uma conexão afim $\nabla$ em uma variedade diferenciável $M$ é uma aplicação

$$
\nabla: \mathfrak{X}(M) \times \mathfrak{X}(M) \rightarrow \mathfrak{X}(M)
$$

que satisfaz

- $\nabla_{f X+g Y} Z=f \nabla_{X} Z+g \nabla_{Y} Z$

- $\nabla_{X}(Y+Z)=\nabla_{X} Y+\nabla_{X} Z$

- $\nabla_{X}(f Y)=f \nabla_{X} Y+X(f) Y$

onde $X, Y, Z \in \mathfrak{X}(M), f, g \in C^{\infty}(M)$.

Definição A.14 A conexão é dita ser compatível com a métrica $\langle$,$\rangle quando$ para toda curva diferenciável c e quaisquer pares de vetores paralelamente propagados $P$ e $P^{\prime}$ ao longo de c tivermos $\left\langle P, P^{\prime}\right\rangle=$ constante.

Proposição A.15 Seja $M$ uma variedade diferenciável. Uma conexão $\nabla$ em $M$ é compativel com a métrica se e somente se para todo par $V$ e $W$ de campos de vetores ao longo da curva diferenciável $c: I \rightarrow M$ tem-se

$$
\frac{d}{d t}\langle V, W\rangle=\left\langle\frac{D V}{d t}, W\right\rangle+\left\langle V, \frac{D W}{d t}\right\rangle
$$

(demonstração em [17]).

Corolário A.16 Uma conexão $\nabla$ em uma variedade $M$ é compatível com a métrica se e somente se

$$
X\langle Y, Z\rangle=\left\langle\nabla_{X} Y, Z\right\rangle+\left\langle Y, \nabla_{X} Z\right\rangle
$$

Definição A.17 Uma conexão afim $\nabla$ em uma variedade diferenciável $M$ é simétrica quando

$$
\nabla_{X} Y-\nabla_{Y} X=[X, Y]
$$

$X, Y \in \mathfrak{X}(M)$. 
Teorema A.18 (Levi - Civita) Dada uma variedade (pseudo-)Riemanniana $M$ existe uma única conexão afim $\nabla$ em $M$ satisfazendo as condições

(i) $\nabla$ é simétrica;

(ii) $\nabla$ é compativel com a métrica.

Conexões que satisfazem as condições $(i)$ e (ii) são ditas conexões de LeviCivita.

Há casos em que se pode ter interesse em trabalhar com conexões que não são simétricas. Nestes casos introduz-se a torção como um mapa entre $\mathfrak{X}(M) \times$ $\mathfrak{X}(M) \rightarrow \mathfrak{X}(M)$ definido por

$$
T(X, Y)=\nabla_{X} Y-\nabla_{Y} X-[X, Y] .
$$

Claramente quando a conexão é simétrica a torção anula-se identicamente.

\section{A.4 Curvaturas}

Definição A.19 A curvatura $R$ de uma variedade Lorentziana (Riemanniana) $M$ é uma correspondência que associa a cada par $X, Y \in \mathfrak{X}(M)$ uma aplicação $R(X, Y) Z: \mathfrak{X}(M) \rightarrow \mathfrak{X}(M)$ dada por

$$
R(X, Y) Z=\nabla_{X} \nabla_{Y} Z-\nabla_{Y} \nabla_{X} Z-\nabla_{[X, Y]} Z
$$

$Z \in \mathfrak{X}(M)$

Proposição A.20 A curvatura $R$ de uma variedade Lorentziana goza das seguintes propriedades:

- $R$ é bilinear em $\mathfrak{X}(M) \times \mathfrak{X}(M)$, isto é,

$$
\begin{aligned}
R\left(f X_{1}+g X_{2}, Y_{1}\right) & =f R\left(X_{1}, Y_{1}\right)+g R\left(X_{2}, Y_{1}\right) \\
R\left(X_{1}, f Y_{1}+g Y_{2}\right) & =f R\left(X_{1}, Y_{1}\right)+g R\left(X_{1}, Y_{2}\right)
\end{aligned}
$$

$f, g \in C^{\infty}(M), X_{1}, X_{2}, Y_{1}, Y_{2} \in \mathfrak{X}(M)$.

- Para todo par $X, Y \in \mathfrak{X}(M)$ o operador curvatura $R(X, Y): \mathfrak{X}(M) \rightarrow$ $\mathfrak{X}(M)$ é linear, isto é,

$$
\begin{array}{r}
R(X, Y)(Z+W)=R(X, Y) Z+R(X, Y) W \\
R(X, Y)(f Z)=f R(X, Y) Z
\end{array}
$$

$f \in \mathcal{D}(M), Z, W \in \mathfrak{X}(M)$. 
118 Teoremário

A demonstração desta proposição segue das propriedades da conexão.

Proposição A.21 (Primeira identidade de Bianchi): $R(X, Y) Z+R(Y, Z) X+$ $R(Z, X) Y=0$.

A demonstração consiste em expandir os fatores, da propriedade de simetria da conexão e da identidade de Jacobi.

Proposição A.22 Considere o objeto $(X, Y, Z, T):=\langle R(X, Y) Z, T\rangle$. Valem as identidades:

(i) $(X, Y, Z, T)+(Y, Z, X, T)+(Z, X, Y, T)=0$

(ii) $(X, Y, Z, T)=-(Y, X, Z, T)$

(iii) $(X, Y, Z, T)=-(X, Y, T, Z)$

(iv) $(X, Y, Z, T)=(Z, T, X, Y)$

\section{Demonstração}

(i) Segue da identidade de Bianchi.

(ii) Conseqüência da definição.

(iii) Devemos primeiro notar que $(X, Y, Z, T)=-(X, Y, T, Z)$ se, e somente se, $(X, Y, Z, Z)=0$, pois $0=(X, Y,(Z+T),(Z+T))=(X, Y, Z, T)+$ $(X, Y, T, Z)$. Por definição,

$$
(X, Y, Z, Z)=\left\langle\nabla_{X} \nabla_{Y} Z-\nabla_{Y} \nabla_{X} Z-\nabla_{[X, Y]} Z, Z\right\rangle
$$

$\operatorname{mas}\left\langle\nabla_{Y} \nabla_{X} Z, Z\right\rangle=Y\left\langle\nabla_{X} Z, Z\right\rangle-\left\langle\nabla_{X} Z, \nabla_{Y} Z\right\rangle$ e $\left.\left.\left\langle\nabla_{[X, Y]} Z, Z\right\rangle=1 / 2[X, Y]\right\rangle Z, Z\right\rangle$ como consequência de (A.2). Daí,

$$
\begin{aligned}
(X, Y, Z, Z) & =Y\left\langle\nabla_{X} Z, Z\right\rangle-X\left\langle\nabla_{Y} Z, Z\right\rangle+\frac{1}{2}[X, Y]\langle Z, Z\rangle \\
& =\frac{1}{2} Y(X\langle Z, Z\rangle)-\frac{1}{2} X(Y\langle Z, Z\rangle)+\frac{1}{2}[X, Y]\langle Z, Z\rangle \\
& =\frac{1}{2}[Y, X]\langle Z, Z\rangle+\frac{1}{2}[X, Y]\langle Z, Z\rangle=0
\end{aligned}
$$

como queríamos mostrar.

(iv) Segue de escrever 4 vezes a identidade de Bianchi alterando a posição dos campos e adicionar ou subtraí-los de maneira conveniente. 
Importantemente relacionado ao tensor de curvatura de Riemann está o tensor de Weyl [75]

$$
R_{\alpha \mu \beta \nu}=: C_{\alpha \mu \beta \nu}+\frac{2}{n-2}\left(g_{\alpha[\beta} R_{\nu] \mu}-g_{\mu[\beta} R_{\nu] \alpha}\right)-\frac{2}{(n-1)(n-2)} R g_{\alpha[\beta} g_{\nu] \mu}
$$

\section{A.4.1 Espaços de Friedmann-Lemaître-Robertson-Walker e espaços com curvatura constante}

Passamos agora a alguns resultados sobre curvatura em espaços de curvatura constante. As demonstrações podem ser encontradas em [76]. Vamos dar algumas definições e resultados sobre espaços-tempo de Friedmann-LemaîtreRobertson-Walker.

Definição A.23 Um espaço-tempo é dito ser de Friedmann-Lemaître-RobertsonWalker (FLRW) se for um modelo 3+1-dimensional satisfazendo o princípio cosmológico, isto é, a métrica escreve-se no produto $M \times \mathbb{R}$, com $M$ uma variedade tridimensional,

$$
g=-d t^{2}+{ }^{(3)} g_{t}
$$

onde ${ }^{(3)} g_{t}$ é uma métrica Riemanninana de curvatura constante, independente de $t$ e globalmente homogênea.

Uma vez motivada a divisão $3+1$ das métricas em que estamos interessados, podemos proceder com nossa coleção de resultados.

Definição A.24 A curvatura seccional em um ponto $x$ de uma variedade Riemanniana $(M, g)$, relativa a um 2-subplano $P$ do espaço tangente $T_{x}$, é um número, independente da escolha de $X$ e $Y$, vetores linearmente independentes em $P$,

$$
K(P):=\frac{\langle R(X, Y) X, Y\rangle}{\langle X, X\rangle\langle Y, Y\rangle-\langle X, Y\rangle^{2}}
$$

Definição A.25 Uma variedade Riemanniana $(M, g)$ é dita isotrópica em $x$ se sua curvatura seccional $K(P)$ for independente da escolha do 2-plano $P$ em $x$.

O seguinte teorema caracteriza a isotropia em termos do tensor de Riemann: 
Teorema A.26 $(M, g)$ é isotrópico em $x$ se e somente se seu tensor de Riemann em $x$ toma a forma

$$
R_{i j k l}(x)=K(x)\left(g_{i k} g_{j l}-g_{j k} g_{i l}\right) .
$$

Além disso, $R_{i j}=(n-1) K g_{i j}$ e $R=n(n-1) K$.

Definição A.27 Uma variedade Riemanniana $(M, g)$ é dito ser um espaço de curvatura Riemanniana constante se é isotrópico em cada ponto $x \in M$ e se sua curvatura seccional for constante em $M$.

Isotropia, que é um importante ingrediente em modelos cosmológicos, tem uma importante relação com espaços de curvatura constante, como estabelecido no seguinte teorema:

Teorema A.28 Se uma variedade Riemanniana é isotrópica em cada ponto, então é um espaço de curvatura constante.

Consideremos ainda o seguinte importante lema [76]:

Lema A.29 Seja $(M, g)$ um espaço de curvatura constante K. Então

(i) $(M, g)$ é localmente conformalmente plano.

(ii) No domínio de cada carta há um sistema de coordenadas $x^{i}$ no qual a métrica toma a forma

$$
g=\frac{\left(d x^{1}\right)^{2}+\ldots+\left(d x^{n}\right)^{2}}{1+\frac{K}{4}\left[\left(x^{1}\right)^{2}+\ldots+\left(x^{n}\right)^{2}\right]^{2}}
$$

Segue desse lema que o tensor de Weyl anula-se identicamente nestes espaços. Enumeramos ainda, por completeza, os seguintes notáveis resultados:

Teorema A.30 Uma variedade Riemanniana com curvatura constante $K$ é localmente isométrica

(i) ao espaço Euclideano $E^{n}$, se $K=0$.

(ii) a uma esfera de raio $K^{-1 / 2}$, subvariedade

$$
\sum_{A=1, \ldots, n+1}\left(x^{A}\right)^{2}=K^{-1}
$$

do espaço Euclideano $E^{n+1}$, se $K>0$. 
(iii) a uma pseudo-esfera de pseudo-raio $|K|^{-1 / 2}$, a subvariedade conexa difeomorfa $a \mathbb{R}^{n}$,

$$
\sum_{i=1, \ldots, n}-\left(x^{0}\right)^{2}+\left(x^{i}\right)^{2}=|K|^{-1}, \quad x^{0}>0
$$

do espaço de Minkowski $M^{n+1}$ com assinatura $(-,+, \ldots,+)$, se $K<0$.

Corolário A.31 As esferas, os espaços Euclideanos e as pseudo-esferas são espaços homogêneos.

\section{A.5 Bases não-coordenadas e equações de estrutura de Cartan}

Dado um sistema de coordenadas locais em uma carta de uma variedade $(M, g)$ em torno de um ponto $p$, sabemos que há canonicamente associada uma base local em $T_{p} M$ dada pelos vetores $\partial / \partial x^{\mu}$ e consequentemente uma base em $T_{p}^{*} M$ formada pelos vetores $d x^{\mu}$. Estas bases são ditas bases coordenadas. Podemos, no entanto, utilizar uma espécie de procedimento de Gram-Schmidt aos vetores da base do espaço tangente, isto é, consideraremos a transformação:

$$
\hat{e}_{\alpha}=e_{\alpha}^{\mu} \frac{\partial}{\partial x^{\mu}} \quad\left\{e_{\alpha}^{\mu}\right\} \in \mathrm{GL}(m, \mathbb{R}),
$$

ou, em outras palavras, $\left\{\hat{e}_{\alpha}\right\}$ é um conjunto de vetores obtidos de $\left\{e_{\mu}\right\}$ por uma $\mathrm{GL}(m, \mathbb{R})$-rotação ${ }^{5}$, preservando a orientação. Requeremos que o resultado desta transformação seja fazer com que os vetores $\left\{\hat{e}_{\alpha}\right\}$ formem uma base ortonormal respeito a $g$, ou seja,

$$
g\left(\hat{e}_{\alpha}, \hat{e}_{\beta}\right)=e_{\alpha}^{\mu} e_{\beta}^{\nu} g_{\mu \nu}=\eta_{\alpha \beta} .
$$

Naturalmente podemos resolver (A.16) em termos das componentes da métrica:

$$
g_{\mu \nu}=e^{\alpha}{ }_{\mu} e_{\nu}^{\beta} \eta_{\alpha \beta}
$$

onde $e^{\alpha}{ }_{\mu}$ é a inversa de $e_{\alpha}^{\mu}$.

Seja $V$ um vetor no espaço tangente em um ponto $p$ de $M$. Em um sistema de coordenadas locais certamente vale $V=V^{\mu} e_{\mu}=V^{\alpha} \hat{e}_{\alpha}=V^{\alpha} e_{\alpha}{ }^{\mu} e_{\mu}$, ou seja,

$$
V^{\mu}=V^{\alpha} e_{\alpha}^{\mu} \quad V^{\alpha}=e^{\alpha}{ }_{\mu} V^{\mu} .
$$

${ }^{5} G L(m, \mathbb{R})$ é o grupo das matrizes reais $m \times m$ com determinante não-nulo. 


\section{Teoremário}

Podemos também introduzir a base dual $\left\{\hat{\theta}^{\alpha}\right\}$ definida por $\left\langle\hat{\theta}^{\alpha}, \hat{e}_{\beta}\right\rangle=\delta_{\beta}^{\alpha}$. $\hat{\theta}^{\alpha}$ escreve-se como

$$
\hat{\theta}^{\alpha}=e^{\alpha}{ }_{\mu} d x^{\mu}
$$

e em termos dela podemos expressar a métrica como:

$$
g=g_{\mu \nu} d x^{\mu} \otimes d x^{\nu}=\delta_{\alpha \beta} \hat{\theta}^{\alpha} \otimes \hat{\theta}^{\beta} .
$$

As bases $\left\{\hat{e}_{\alpha}\right\}$ e $\left\{\hat{\theta}^{\alpha}\right\}$ são chamadas de bases não-coordenadas. Os coeficientes $e_{\alpha}^{\mu}$ são chamados de vierbeine em espaços quadridimensionais, vielbeine em espaços de maior dimensão e zweibeine no caso de termos uma variedade bi-dimensional ${ }^{6}$.

Uma das propriedades fundamentais das bases não-coordenadas é que essas têm colchete de Lie não nulo, ou seja,

$$
\left.\left[\hat{e}_{\alpha}, \hat{e}_{\beta}\right]\right|_{p}=\left.c_{\alpha \beta}^{\gamma}(p) \hat{e}_{\gamma}\right|_{p}
$$

onde $c_{\alpha \beta}^{\gamma}(p)=e_{\gamma}{ }^{\nu}\left[e_{\alpha}{ }^{\mu} \partial_{\mu} e_{\beta}{ }^{\nu}-e_{\beta}{ }^{\mu} \partial_{\mu} e_{\alpha}{ }^{\nu}\right](p)$.

Utilizamos (A.18) para expressar em coordenadas a curvatura de Riemann (A.5) e a torção (A.4):

$$
\begin{aligned}
T_{\beta \gamma}^{\alpha} & =\Gamma_{\beta \gamma}^{\alpha}-\Gamma_{\gamma \beta}^{\alpha}-c_{\beta \gamma}{ }^{\alpha} \\
R_{\beta \gamma \delta}^{\alpha} & =\hat{e}_{\gamma} \Gamma_{\delta \beta}^{\alpha}-\hat{e}_{\delta} \Gamma_{\gamma \beta}^{\alpha}+\Gamma_{\delta \beta}^{\varepsilon} \Gamma_{\gamma \varepsilon}^{\alpha}-\Gamma_{\gamma \beta}^{\varepsilon} \Gamma_{\delta \epsilon}^{\alpha}-c_{\gamma \delta}{ }^{\varepsilon} \Gamma_{\varepsilon \beta}^{\alpha},
\end{aligned}
$$

onde os coeficientes da conexão com respeito à base $\left\{\hat{e}_{\alpha}\right\}$ são escritos como $\nabla_{\hat{e}_{\alpha}} \hat{e}_{\beta}=\Gamma_{\alpha \beta}^{\gamma} \hat{e}_{\gamma}$.

Consideremos ainda a 1-forma de conexão, definida pela relação:

$$
\omega_{\beta}^{\alpha}=\Gamma_{\gamma \beta}^{\alpha} \hat{\theta}^{\gamma} .
$$

As 1-formas de conexão satisfazem importantes relações chamadas equações de estrutura de Cartan, cuja demonstração pode ser encontrada, por exemplo, em [36] ou [77]. Estas equações são extremamente convenientes em cálculos

\footnotetext{
${ }^{6} \mathrm{Em}$ alemão a palavra bein significa perna. Logo um vierbeine é um objeto com quatro pernas (vier significa quatro), um zweibeine é um objeto com duas pernas (zwei significa dois) e um vielbeine um objeto com muitas pernas, já que a palavra viel significa muitos. Como o plural da palavra bein em alemão é beine, para manter consistência escrevemos, por exemplo, vierbeine e não vierbeins ou vierbein como eventualmente lê-se em alguns textos.
} 
envolvendo o tensor de curvatura e é este o motivo principal de as introduzirmos aqui. Como se tratam de duas relações, elas são - não surpreendentemente conhecidas como primeira e segunda equação de estrutura de Cartan:

$$
\begin{gathered}
d \hat{\theta}^{\alpha}+\omega_{\beta}^{\alpha} \wedge \hat{\theta}^{\beta}=T^{\alpha} \\
d \omega^{\alpha}{ }_{\beta}+\omega_{\gamma}^{\alpha} \wedge \omega_{\beta}^{\gamma}=R_{\beta}^{\alpha},
\end{gathered}
$$

onde introduzimos a 2-forma de torção $T^{\alpha}=\frac{1}{2} T^{\alpha}{ }_{\beta \gamma} \hat{\theta}^{\beta} \wedge \hat{\theta}^{\gamma}$ e a 2-forma de curvatura $R_{\beta}^{\alpha}=\frac{1}{2} R_{\beta \gamma \delta}^{\alpha} \hat{\theta}^{\gamma} \wedge \hat{\theta}^{\delta}$.

Como notamos, quando a conexão é de Levi-Civita, a torção anula-se identicamente. Neste caso pode-se mostrar que a 1-forma de conexão satisfaz

$$
\omega_{\alpha \beta}=-\omega_{\beta \alpha}
$$

onde $\omega_{\alpha \beta}=\delta_{\alpha \gamma} \omega_{\beta}^{\gamma}$.

\section{A.6 Geodésicas}

Definição A.32 Uma curva parametrizada $\gamma: I \rightarrow M$ é uma geodésica em $t_{0} \in I$ se $\frac{D}{d t}\left(\frac{d \gamma}{d t}\right)=0$ no ponto $t_{0}$; se $\gamma$ é geodésica em $t \in I$, dizemos que $\gamma$ é uma geodésica. Se $[a, b] \in I$ e $\gamma: I \rightarrow M$ é uma geodésica, a restrição de $\gamma$ a $[a, b]$ é chamada de geodésica ligando $\gamma(a)$ a $\gamma(b)$.

Lema A.33 (Homogeneidade de uma geodésica) Se a geodésica $\gamma(t, q, v)$ ( $t$ é parâmetro e q e $v$, condições iniciais) está definida no intervalo $(-\delta, \delta)$, então a geodésica $\gamma(t, q, a v), a \in \mathbb{R}, a>0$, está definida no intervalo $\left(-\frac{\delta}{a}, \frac{\delta}{a}\right)$ e

$$
\gamma(t, q, a v)=\gamma(a t, q, v)
$$

Um importante conceito relacionado a geodésicas é o de aplicação exponencial.

Definição A.34 Seja $B_{\varepsilon}(0) \subset T_{p} M$ a bola aberta de raio $\epsilon$ centrada na origem de $T_{p} M$. Define-se

$$
\exp _{p}: B_{\varepsilon}(0) \subset T_{p} M \rightarrow M
$$

por $\exp _{p}(v)=\gamma(1, p, v)=\gamma\left(a, p, \frac{v}{a}\right)$, sendo que a segunda igualdade segue do lema de homogeneidade de uma geodésica. 
Geometricamente $\exp _{p}(v)$ é o ponto de $M$ obtido percorrendo-se um comprimento igual a $a$, a partir de $p$, sobre a geodésica que passa por $p$ com velocidade $\frac{v}{a}$. Mostra-se que para todo $p \in M$ existe um $\varepsilon>0$ tal que $\exp _{p}: B_{\varepsilon}(0) \subset T_{p} M \rightarrow M$ é um difeomorfismo de $B_{\varepsilon}(0)$ sobre um aberto de $M$.

Finalizando esta seção, um dos mais importantes resultados sobre a aplicação exponencial:

Lema A.35 (de Gauss) Sejam $p \in M$ e $v \in T_{p} M$ tal que $\exp _{p} v$ esteja definida. Seja $w \in T_{p} M \sim T_{v}\left(T_{p} M\right)$. Então

$$
\left\langle\left(\exp _{p}\right)_{v}(v),\left(d \exp _{p}\right)_{v}(w)\right\rangle=\langle v, w\rangle .
$$

\section{A.7 Campos de Jacobi}

Definição A.36 Seja $\gamma:[0, a] \rightarrow M$ uma geodésica em $M$. Um campo de vetores $J$ ao longo de $\gamma$ é um campo de Jacobi se satisfaz a equação de Jacobi:

$$
\frac{D^{2} J}{d t^{2}}+R\left(\gamma^{\prime}(t), J(t)\right) \gamma^{\prime}(t)=0
$$

para $t \in[0, a]$.

Uma observação simples é que dada uma geodésica $\gamma(t)$ em $M, \gamma^{\prime}(t)$ e $t \gamma^{\prime}(t)$ são campos de Jacobi ao longo de $\gamma$, pois

$$
\frac{D^{2}}{d t^{2}}\left(\gamma^{\prime}(t)\right)+R\left(\gamma^{\prime}(t), \gamma^{\prime}(t)\right) \gamma^{\prime}(t)=0
$$

$$
\begin{gathered}
\frac{D^{2}}{d t^{2}}\left(t \gamma^{\prime}(t)\right)=\frac{D}{d t}\left(\frac{D}{d t}\left(t \gamma^{\prime}(t)\right)\right)=\frac{D}{d t}\left(\gamma^{\prime}(t)+t \frac{D}{d t} \gamma^{\prime}(t)\right)=0 \\
R\left(\gamma^{\prime}(t), t \gamma^{\prime}(t)\right) \gamma^{\prime}(t)=t R\left(\gamma^{\prime}(t), \gamma^{\prime}(t)\right) \gamma^{\prime}(t)=0
\end{gathered}
$$

Daremos agora uma importante caracterização de campos de Jacobi em termos da aplicação exponencial. Dados $p \in M$ e $w \in T_{v}\left(T_{p} M\right)$, podemos construir um campo de Jacobi ao longo da geodésica $\gamma:[0,1] \rightarrow M$, dada por $\gamma(t)=\exp _{p} t v$. Para isto considere a superfície parametrizada por $f(t, s)=$ $\exp _{p} t v(s)$, onde $v(s)$ é uma curva em $T_{p} M \operatorname{com} v(0)=v, v^{\prime}(0)=w$, e fazemos 
$J(t)=\frac{\partial f}{\partial s}(t, 0)$. Nota-se que $J(0)=0$. Esta é, com efeito, a única maneira de construir campos de Jacobi ao longo de $\gamma(t)$ com $J(0)=0$. Notemos que, para $s=0$,

$$
\begin{aligned}
\frac{D}{d t} \frac{\partial f}{\partial s}= & \frac{D}{\partial t}\left(\left(\operatorname{dexp}_{p}\right)_{t v}(t w)\right)=\frac{D}{\partial t}\left(t\left(\operatorname{dexp}_{p}\right)_{t v}(w)\right) \\
& =\left(\operatorname{dexp}_{p}\right)_{t v}(w)+t \frac{D}{\partial t}\left(\left(\operatorname{dexp}_{p}\right)_{t v}(w)\right)
\end{aligned}
$$

Portanto, para $t=0$,

$$
\frac{D J}{d t}(0)=\left(\exp _{p}\right)_{0}(w)=w
$$

Podemos, então, enunciar essa caracterização:

Proposição A.37 Seja $\gamma(t):[0, a] \rightarrow M$ uma geodésica $p=\gamma(0)$. Então um campo de Jacobi $J$ ao longo de $\gamma$ com $J(0)=0$ é dado por

$$
J(t)=\left(\operatorname{dexp}_{p}\right)_{t \gamma^{\prime}(0)}\left(t J^{\prime}(0)\right), \quad t \in[0, a] .
$$

Retomando o fato da aplicação exponencial ser um difeomorfismo local entre uma vizinhança da origem de $T_{p} M$ e uma vizinhança de $p \in M$, sabemos que a diferencial desta aplicação é não-singular na vizinhança do vetor nulo e portanto, nesta vizinhança, transformará vetores linearmente independentes em vetores linearmente independentes. Além disso, se a dimensão de $M$ é $n$, existem exatamente $n$ campos de Jacobi linearmente independentes ao longo de uma geodésica $\gamma:[0, a] \rightarrow M$ que se anulam em $\gamma(0)$. Isso decorre do fato que os campos de Jacobi $J_{1}, \ldots, J_{k}$ com $J_{i}(0)=0$ são linearmente independentes se e somente se $J_{1}^{\prime}(0), \ldots, J_{k}^{\prime}(0)$ o são.

Para estabelecer uma nomenclatura, consideremos a definição:

Definição A.38 Seja $\gamma:[0, a] \rightarrow M$ uma geodésica. O ponto $\gamma\left(t_{0}\right)$ é conjugado de $\gamma(0)$ ao longo de $\gamma, t_{0} \in(0, a]$, se existe um campo de Jacobi $J$ ao longo de $\gamma$, não identicamente nulo, com $J(0)=0=J\left(t_{0}\right)$. O número de tais campos linearmente independentes é a multiplicidade do ponto conjugado $\gamma\left(t_{0}\right)$.

Um ponto $q=\gamma\left(t_{0}\right), t_{0} \in(0, a]$ é conjugado de $p=\gamma(0)$ se e somente se $v_{0}=t_{0} \gamma^{\prime}(0)$ é um ponto crítico da aplicação exponencial e a multiplicidade deste ponto é igual à dimensão do núcleo de $\left(d \exp _{p}\right)_{v_{0}}$. 
126 Teoremário

Os seguintes resultados serão também essenciais:

Proposição A.39 Seja J um campo de Jacobi ao longo da geodésica $\gamma$ : $[0, a] \rightarrow M$. Então

$$
\left\langle J(t), \gamma^{\prime}(t)\right\rangle=\left\langle J^{\prime}(0), \gamma^{\prime}(0)\right\rangle t+\left\langle J(0), \gamma^{\prime}(0)\right\rangle \quad t \in[0, a]
$$

\section{Demonstração}

Da equação de Jacobi e do fato de $\gamma$ ser geodésica,

$$
\left\langle J^{\prime}(t), \gamma^{\prime}(t)\right\rangle^{\prime}=\left\langle J^{\prime \prime}(t), \gamma^{\prime}(t)\right\rangle=-\left\langle R\left(\gamma^{\prime}, J\right) \gamma^{\prime}, \gamma^{\prime}\right\rangle=0
$$

como consequência do terceiro item da Proposição A.22. Portanto, $\left\langle J^{\prime}(t), \gamma^{\prime}(t)\right\rangle=$ $\left\langle J^{\prime}(0), \gamma^{\prime}(0)\right\rangle$. Além disso,

$$
\left\langle J(t), \gamma^{\prime}(t)\right\rangle^{\prime}=\left\langle J^{\prime}(t), \gamma^{\prime}(t)\right\rangle=\left\langle J^{\prime}(0), \gamma^{\prime}(0)\right\rangle \text {. }
$$

Integrando-se esta última equação obtemos o resultado que desejávamos demonstrar.

Corolário A.40 Suponha que $J(0)=0$. Então $\left\langle J(t), \gamma^{\prime}(t)\right\rangle \equiv 0$ se e somente se $\left\langle J^{\prime}(0), \gamma^{\prime}(0)\right\rangle=0$.

este corolário também pode ser visto como conseqüência direta do Lema de Gauss (Lema A.35). 


\section{Apêndice B \\ Trajetórias em espaços-tempo perturbados}

Para calcular a deformação dos feixes do fótons quando se propagam sob o efeito da curvatura, devemos saber a priori quais as geodésicas correspondentes aos fótons centrais dos feixes. Conhecer estas geodésicas pode ser uma tarefa bastante complicada. Em alguns casos, entretanto, podemos eventualmente saber as geodésicas nulas em um dado espaço-tempo e isto nos sirva para determinar as geodésicas em um outro espaço-tempo obtido do primeiro por aplicações de perturbações. Desenvolveremos nesta seção uma maneira de executar tal procedimento. Este desenvolvimento associado às características da matriz de maré óptica em espaços conformalmente planos constituem a base para a justificativa da aproximação de Born no estudo de lentes gravitacionais.

Consideremos um espaço-tempo que pode ser tratado como um espaçotempo base sobre o qual aplicam-se perturbações e veremos como as geodésicas neste espaço sujeito a perturbações relacionam-se às do espaço original. Inspirados em [78], tomemos $g_{\mu \nu}=g_{\mu \nu}^{(0)}+h_{\mu \nu} \operatorname{com} g_{\mu \nu}^{(0)}$ sendo a métrica do espaço base e $h_{\mu \nu}$ a perturbação. Como usual em teoria de perturbação, usa-se a métrica do espaço não-perturbado para subir e baixar índices.

Os coeficientes da conexão de $g_{\mu \nu}$, que são dados por

$$
\Gamma_{\alpha \beta}^{\mu}=\frac{1}{2} g^{\mu \sigma}\left(g_{\sigma \beta, \alpha}+g_{\alpha \sigma, \beta}-g_{\alpha \beta, \sigma}\right),
$$

podem ser separados em um termo de ordem zero e um de primeira ordem em $h$ :

$$
\Gamma_{\alpha \beta}^{\mu}=\Gamma_{\alpha \beta}^{(0) \mu}+\Gamma_{\alpha \beta}^{(1) \mu},
$$

onde

$$
\Gamma_{\alpha \beta}^{(0) \mu}=\frac{1}{2} g^{(0) \mu \sigma}\left(g_{\sigma \beta, \alpha}^{(0)}+g_{\alpha \sigma, \beta}^{(0)}-g_{\alpha \beta, \sigma}^{(0)}\right)
$$

e

$$
\Gamma_{\alpha \beta}^{(1) \mu}=\frac{1}{2} g^{(0) \mu \sigma}\left(h_{\sigma \beta, \alpha}+h_{\alpha \sigma, \beta}-h_{\alpha \beta, \sigma}\right)-\frac{1}{2} g^{(0) \mu \sigma}\left(g_{\sigma \beta, \alpha}^{(0)}+g_{\alpha \sigma, \beta}^{(0)}-g_{\alpha \beta, \sigma}^{(0)}\right),
$$


128 Trajetórias em espaços-tempo perturbados

ou seja,

$$
\Gamma_{\alpha \beta}^{(1) \mu}=\frac{1}{2} g^{(0) \mu \sigma}\left(h_{\sigma \beta ; \alpha}+h_{\alpha \sigma ; \beta}-h_{\alpha \beta ; \sigma}\right) .
$$

O ponto-e-vírgula acima indica derivação covariante com relação à conexão de $g_{\mu \nu}^{(0)}$.

Seja $x^{(0) \mu}(\lambda)$ uma geodésica no espaço-tempo base com parâmetro afim $\lambda$. $x^{(0) \mu}(\lambda)$ costuma ser dita "a trajetória não perturbada". Por hipótese $x^{(0) \mu}(\lambda)$ satisfaz

$$
\ddot{x}^{(0) \mu}+\Gamma_{\alpha \beta}^{(0) \mu}\left(x^{(0)}\right) \dot{x}^{(0) \alpha} \dot{x}^{(0) \beta}=0,
$$

$\operatorname{com}\left(\cdot=\frac{d}{d \lambda}\right)$ e $\Gamma_{\alpha \beta}^{(0) \mu}\left(x^{(0)}\right)$ significando que os símbolos da conexão são calculados sobre a trajetória não-perturbada.

Consideremos agora a expressão:

$$
x^{\mu}(\lambda)=x^{(0) \mu}(\lambda)+x^{(1) \mu}(\lambda) .
$$

Aqui $x^{\mu}(\lambda)$ e $x^{(1) \mu}(\lambda)$ não são especificados e a equação (B.4) pode ser entendida como definidora tanto de um quanto de outro, uma vez que um deles seja dado. Vamos derivar condições em $x^{(1) \mu}(\lambda)$ que serão necessárias e suficientes para que $x^{\mu}(\lambda)$ seja uma geodésica no espaço perturbado. No que segue, vamos truncar as expansões na primeira ordem em termos perturbados, ou seja, vamos descartar produtos envolvendo mais de um termo do tipo $x^{(1) \mu}(\lambda)$ ou $\dot{x}^{(1) \mu}(\lambda)$.

Derivando duas vezes (B.4) e usando (B.3), temos

$$
\ddot{x}^{\mu}=-\Gamma_{\alpha \beta}^{(0) \mu}\left(x^{(0)}\right) \dot{x}^{(0) \alpha} \dot{x}^{(0) \beta}+\ddot{x}^{(1) \mu} .
$$

Por outro lado, se $x^{\mu}(\lambda)$ é uma geodésica parametrizada de modo afim no espaço-tempo perturbado, deve valer

$$
\ddot{x}^{\mu}=-\Gamma_{\alpha \beta}^{\mu}(x) \dot{x}^{\alpha} \dot{x}^{\beta}=-\Gamma_{\alpha \beta}^{(0) \mu}(x)\left(\dot{x}^{(0) \alpha} \dot{x}^{(0) \beta}+2 \dot{x}^{(0) \alpha} \dot{x}^{(1) \beta}\right)-\Gamma_{\alpha \beta}^{(1) \mu}(x) \dot{x}^{(0) \alpha} \dot{x}^{(0) \beta} .
$$

Podemos agora fazer uma expansão dos coeficientes da conexão em torno da trajetória não-perturbada:

$$
\begin{gathered}
\Gamma_{\alpha \beta}^{(0) \mu}(x)=\Gamma_{\alpha \beta}^{(0) \mu}\left(x^{(0)}\right)+\Gamma_{\alpha \beta, \tau}^{(0) \mu}\left(x^{(0)}\right) x^{(1) \tau}+\ldots \\
\Gamma_{\alpha \beta}^{(1) \mu}(x)=\Gamma_{\alpha \beta}^{(1) \mu}\left(x^{(0)}\right)+\ldots
\end{gathered}
$$


Substituindo isso em (B.6), temos

$$
\begin{aligned}
\ddot{x}^{\mu}= & -\Gamma_{\alpha \beta}^{(0) \mu}\left(x^{(0)}\right) \dot{x}^{(0) \alpha} \dot{x}^{(0) \beta}-\Gamma_{\alpha \beta}^{(1) \mu}\left(x^{(0)}\right) \dot{x}^{(0) \alpha} \dot{x}^{(0) \beta} \\
& -2 \Gamma_{\alpha \beta}^{(0) \mu}\left(x^{(0)}\right) \dot{x}^{(0) \alpha} \dot{x}^{(1) \beta}-\Gamma_{\alpha \beta, \tau}^{(0) \mu}\left(x^{(0)}\right) \dot{x}^{(0) \alpha} \dot{x}^{(0) \beta} x^{(1) \tau} .
\end{aligned}
$$

Comparando as equações (B.7) e (B.5), concluimos que $x^{\mu}(\lambda)$, definido pela equação (B.4), será uma geodésica parametrizada de modo afim no espaçotempo perturbado contanto que $x^{(1) \mu}(\lambda)$ satisfaça o sistema de quatro equações diferenciais acopladas

$$
\left(\frac{d^{2}}{d \lambda^{2}}+A \frac{d}{d \lambda}+B\right) x^{(1)}=f
$$

onde as matrizes $4 \times 4 A$ e $B$ e o vetor (de quatro componentes) $f$ são definidos por

$$
\begin{gathered}
A_{\alpha}^{\mu}=2 \Gamma_{\tau \alpha}^{(0) \mu} k^{(0) \tau}, \\
B_{\alpha}^{\mu}=\Gamma_{\tau \sigma, \alpha}^{(0) \mu} k^{(0) \tau} k^{(0) \sigma}, \\
f=-\Gamma_{\tau \sigma}^{(1) \mu} k^{(0) \tau} k^{(0) \sigma},
\end{gathered}
$$

onde escrevemos $\dot{x}^{(0)}$ como $k^{(0)}$. Devemos também enfatizar que a equação (B.8) vale ao longo de algum segmento da trajetória não-perturbada e gera soluções para a separação $x^{(1)}$ entre a trajetória perturbada e a não-perturbada. Veremos agora que estamos no fundo tratando novamente da equação de Jacobi.

Denotemos por $\frac{D}{d \lambda}$ a derivada covariante ao longo da curva $x^{(0)}$ com a conexão do espaço-tempo base. Com isso, para um vetor arbitrário $v$,

$$
\frac{D}{d \lambda} v^{\mu}=\frac{d v^{\mu}}{d \lambda}+\Gamma_{\alpha \beta}^{(0) \mu} k^{(0) \alpha} v^{\beta}
$$

e

$$
\begin{aligned}
\frac{D^{2}}{d \lambda^{2}} v^{\mu}= & \frac{d^{2} v^{\mu}}{d \lambda^{2}}+\Gamma_{\alpha \beta, \sigma}^{(0) \mu} k^{(0) \alpha} k^{(0) \sigma} v^{\beta}+\Gamma_{\alpha \beta}^{(0) \mu} \frac{d k^{(0) \alpha}}{d \lambda} v^{\beta} \\
& +2 \Gamma_{\alpha \beta}^{(0) \mu} k^{(0) \alpha} \frac{d v^{\beta}}{d \lambda}+\Gamma_{\alpha \beta}^{(0) \mu} k^{(0) \alpha} \Gamma_{\sigma \rho}^{(0) \beta} k^{(0) \sigma} v^{\rho} .
\end{aligned}
$$

Usando a equação de geodésica para $k^{(0)}$, temos 


$$
\begin{aligned}
\frac{D^{2}}{d \lambda^{2}} v^{\mu}= & \frac{d^{2} v^{\mu}}{d \lambda^{2}}+2 \Gamma_{\alpha \beta}^{(0) \mu} k^{(0) \alpha} \frac{d v^{\beta}}{d \lambda}+\Gamma_{\alpha \beta, \sigma}^{(0) \mu} k^{(0) \alpha} k^{(0) \sigma} v^{\beta} \\
& +\Gamma_{\alpha \beta}^{(0) \mu} \Gamma_{\sigma \rho}^{(0) \alpha} k^{(0) \beta} k^{(0) \sigma} v^{\rho}-\Gamma_{\alpha \beta}^{(0) \mu} \Gamma_{\sigma \rho}^{(0) \alpha} k^{(0) \sigma} k^{(0) \rho} v^{\beta} .
\end{aligned}
$$

O tensor de Riemann para o espaço-tempo base pode ser escrito como

$$
R_{\alpha \beta \sigma}^{(0) \mu}=\left(\Gamma_{\alpha \sigma, \beta}^{(0) \mu}-\Gamma_{\alpha \beta, \sigma}^{(0) \mu}+\Gamma_{\beta \rho}^{(0) \mu} \Gamma_{\alpha \sigma}^{(0) \rho}-\Gamma_{\sigma \rho}^{(0) \mu} \Gamma_{\alpha \beta}^{(0) \rho}\right) .
$$

Com isso (B.9) pode ser reescrita:

$$
\frac{D^{2}}{d \lambda^{2}} v^{\mu}-R_{\alpha \beta \sigma}^{(0) \mu} k^{(0) \alpha} k^{(0) \beta} v^{\sigma}=\frac{d^{2} v^{\mu}}{d \lambda^{2}}+2 \Gamma_{\alpha \beta}^{(0) \mu} k^{(0) \alpha} \frac{d v^{\beta}}{d \lambda}+\Gamma_{\alpha \beta, \sigma}^{(0) \mu} k^{(0) \alpha} k^{(0) \beta} v^{\sigma} .
$$

Mas o lado direito de (B.11) é justamente o lado esquerdo da equação (B.8), logo o lado esquerdo de (B.11) é igual a $f$, ou seja,

$$
\nabla_{k^{(0)}}^{2} x^{(1)}-R^{(0)}\left(k^{(0)}, x^{(1)}\right) k^{(0)}=f,
$$

que é uma equação de Jacobi não-homogênea. O campo $x^{(1)}$ em (B.12) é chamado de campo de Jacobi. Aqui o termo não-homogêneo na equação funciona como um termo forçante associado à perturbação. 


\section{Apêndice C}

\section{Alguns resultados sobre funções de Bessel}

Vamos aqui estabelecer alguns resultados sobre integrais envolvendo produtos de funções de Bessel esféricas. Inicialmente mostraremos que produtos de um número arbitrário destas funções em uma combinação específica podem ser expressos em termos de integrais sobre produtos de polinômios de Legendre associados. Em um segundo passo mostraremos que um caso especial das integrais que inicialmente tratamos descreve a probabilidade de que uma partícula em vôo aleatório esteja a uma distância dada após um determinado número de mudanças de direção, percorrendo trechos de tamanhos diferentes mas sempre com a mesma velocidade. Aplicaremos esses resultados finalmente à teoria da radiação cósmica de fundo e estudaremos a expansão das densidades de probabilidades encontradas em séries de Fourier-Bessel. Demonstraremos finalmente uma nova identidade satisfeita por funções de Bessel esféricas que havia surgido em [4] mas para qual, tanto quanto pudemos apurar, não se dispunha de demonstração.

\section{C.1 Primeiro grupo de resultados: Relação entre integrais de produtos de funções de Bessel e vôos aleatórios}

Nesta seção mostraremos alguns resultados sobre integrais envolvendo funções de Bessel esféricas e como associar integrais específicas de produtos destas funções com distribuições de probabilidade que tipicamente aparecem em problemas de vôos aleatórios.

\section{C.1.1 Integrais de produtos de funções de Bessel esféricas}

Muito do que será apresentado é baseado na articulação do seguinte resultado:

Lema C.1 Sejam $j_{l}\left(k r_{1}\right)$ e $j_{l}\left(k r_{2}\right)$ funções de Bessel esféricas. Então,

$$
j_{l}\left(k r_{1}\right) j_{l}\left(k r_{2}\right)=\frac{(-1)^{m}}{2} \int_{\left|r_{1}-r_{2}\right|}^{r_{1}+r_{2}} d r k^{m}\left(\frac{r_{1} r_{2}}{r}\right)^{m-1} P_{l}^{-m}(\cos \alpha)(\operatorname{sen} \alpha)^{m} j_{m}(k r)
$$


132 Alguns resultados sobre funções de Bessel

onde $P_{l}^{-m}(\cos \alpha)$ é um polinômio de Legendre associado, $m \geq l$ e o ângulo $\alpha$ e a variável $r$ são relacionadas através da relação $r^{2}=r_{1}^{2}+r_{2}^{2}-2 r_{1} r_{2} \cos \alpha$.

\section{Demonstração}

Sabemos, de [79], que vale:

$$
\frac{j_{m}(k r)}{(k r)^{m}}=\sum_{n=m}^{\infty} \frac{(2 n+1) j_{n}\left(k r_{1}\right) j_{n}\left(k r_{2}\right)}{\left(\left(k r_{1}\right)\left(k r_{2}\right) \operatorname{sen} \alpha\right)^{m}} P_{n}^{m}(\cos \alpha)
$$

$\operatorname{com} r^{2}=r_{1}^{2}+r_{2}^{2}-2 r_{1} r_{2} \cos \alpha$.

Associando a esta equação a relação de ortogonalidade dos polinômios de Legendre associados:

$$
\int_{-1}^{1} P_{l}^{m}(x) P_{l^{\prime}}^{-m} d x=(-1)^{m} \frac{2}{2 l+1} \delta_{l, l^{\prime}},
$$

podemos escrever:

$$
\begin{aligned}
j_{l}\left(k r_{1}\right) j_{l}\left(k r_{2}\right)= & \sum_{l^{\prime}=m}^{\infty} j_{l^{\prime}}\left(k r_{1}\right) j_{l^{\prime}}\left(k r_{2}\right) \delta_{l, l^{\prime}} \\
& =\sum_{l^{\prime}=m}^{\infty}(-1)^{m} \frac{2 l^{\prime}+1}{2} \int_{-1}^{1} d(\cos \alpha) j_{l^{\prime}}\left(k r_{1}\right) j_{l^{\prime}}\left(k r_{2}\right) P_{l^{\prime}}^{m}(\cos \alpha) P_{l}^{-m}(\cos \alpha) \\
& =\frac{(-1)^{m}}{2} \int_{-1}^{1} d(\cos \alpha) P_{l}^{-m}(\cos \alpha)\left(\left(k r_{1}\right)\left(k r_{2}\right) \operatorname{sen} \alpha\right)^{m} \times \\
& \times \sum_{l^{\prime}=m}^{\infty} \frac{\left(2 l^{\prime}+1\right) j_{l^{\prime}}\left(k r_{1}\right) j_{l^{\prime}}\left(k r_{2}\right) P_{l^{\prime}}^{m}(\cos \alpha)}{\left[\left(k r_{1}\right)\left(k r_{2}\right) \operatorname{sen} \alpha\right]^{m}}
\end{aligned}
$$

Fazendo a mudança de variáveis

$$
\int_{-1}^{1} d(\cos \alpha) \rightarrow \int_{\left|r_{1}-r_{2}\right|}^{r_{1}+r_{2}} d r \frac{r}{r_{1} r_{2}}
$$

e usando (C.2) temos:

$$
j_{l}\left(k r_{1}\right) j_{l}\left(k r_{2}\right)=\frac{(-1)^{m}}{2} \int_{\left|r_{1}-r_{2}\right|}^{r_{1}+r_{2}} d r k^{m}\left(\frac{r_{1} r_{2}}{r}\right)^{m-1} P_{l}^{-m}(\cos \alpha)(\operatorname{sen} \alpha)^{m} j_{m}(k r) .
$$

O Lema C.1 permite-nos avaliar a seguinte integral de três funções de Bessel esféricas: 
Lema C.2 Sendo $j_{l}$ e $j_{m}$ funções de Bessel esféricas e $r_{1}, r_{2}, r_{3} \in \mathbb{R}$ vale:

$$
\int_{0}^{\infty} k^{2} \frac{j_{m}\left(k r_{3}\right)}{\left(k r_{3}\right)^{m}} j_{l}\left(k r_{1}\right) j_{l}\left(k r_{2}\right) d k=(-1)^{m} \frac{\pi}{4} \frac{\left(r_{1} r_{2}\right)^{m-1}}{r_{3}^{2 m+1}} P_{l}^{-m}(\cos \alpha) \operatorname{sen}^{m} \alpha
$$

$\operatorname{com} r_{3}^{2}=r_{1}^{2}+r_{2}^{2}-2 r_{1} r_{2} \cos \alpha$.

A Figura C.1 mostra os elementos envolvidos na integral (C.6).

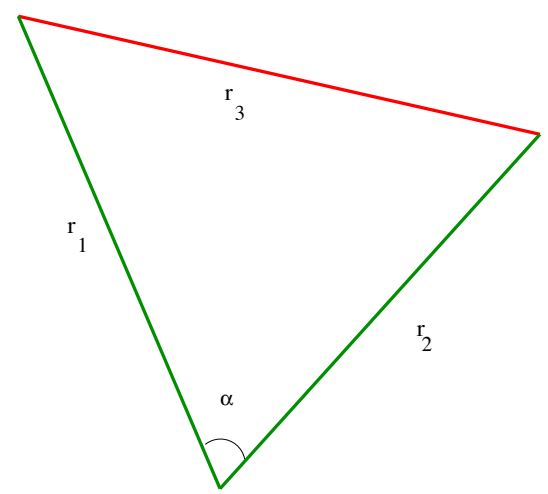

Fig. C.1 Representação geométrica dos elementos que aparecem no enunciado do Lema C.2.

\section{Demonstração:}

$$
\begin{aligned}
\int_{0}^{\infty} k^{2} \frac{j_{m}\left(k r_{3}\right)}{\left(k r_{3}\right)^{m}} j_{l}\left(k r_{1}\right) j_{l}\left(k r_{2}\right) d k= & \frac{(-1)^{m}}{2} \int_{\left|r_{1}-r_{2}\right|}^{r_{1}+r_{2}} d r\left(\frac{r_{1} r_{2}}{r}\right)^{m-1} \frac{1}{r_{3}^{m}} P_{l}^{-m}(\cos \alpha) \operatorname{sen}^{m} \alpha \\
& \times \int_{0}^{\infty} k^{2} j_{m}\left(k r_{3}\right) j_{m}(k r) d k
\end{aligned}
$$

Mas, lembrando a relação de ortogonalidade:

$$
\int_{0}^{\infty} k^{2} j_{L}(a k) j_{L}(b k) d k=\frac{\pi}{2} \frac{b^{L}}{a^{L+2}} \delta(a-b)
$$

verificamos que vale:

$$
\int_{0}^{\infty} k^{2} \frac{j_{m}\left(k r_{3}\right)}{\left(k r_{3}\right)^{m}} j_{l}\left(k r_{1}\right) j_{l}\left(k r_{2}\right) d k=(-1)^{m} \frac{\pi}{4} \frac{\left(r_{1} r_{2}\right)^{m-1}}{r_{3}^{2 m+1}} P_{l}^{-m}(\cos \alpha) \operatorname{sen}^{m} \alpha
$$

contanto que $r^{2}=r_{1}^{2}+r_{2}^{2}-2 r_{1} r_{2} \cos \alpha$.

Como uma conseqüência do Lema C.2, temos: 
134 Alguns resultados sobre funções de Bessel

Lema C.3 Sendo $j_{l}$ e $j_{m}$ funções de Bessel esféricas e $r_{1}, r_{2}, r_{3}, r_{4} \in \mathbb{R}$ vale:

$$
\begin{aligned}
& \int_{0}^{\infty} d k k^{2} \frac{j_{l}\left(k r_{1}\right)}{\left(k r_{1}\right)^{m}} \frac{j_{m}\left(k r_{3}\right)}{\left(k r_{3}\right)^{m}} j_{l}\left(k r_{2}\right) j_{m}\left(k r_{4}\right)=\frac{\pi}{8} \frac{1}{r_{1}^{m} r_{3}^{m}} \int_{\left|r_{1}-r_{2}\right|}^{r_{1}+r_{2}} d r \frac{\left(r_{1} r_{2} r_{3} r_{4}\right)^{m-1}}{r^{2 m}} \times \\
& P_{l}^{-m}(\cos \alpha) \operatorname{sen}^{m} \alpha P_{m}^{-m}\left(\cos \alpha_{1}\right) \operatorname{sen}^{m} \alpha_{1} \\
& \text { com } r^{2}=r_{1}^{2}+r_{2}^{2}-2 r_{1} r_{2} \cos \alpha e r^{2}=r_{3}^{2}+r_{4}^{2}-2 r_{3} r_{4} \cos \alpha_{1} .
\end{aligned}
$$

A representação geométrica dos elementos envolvidos na integral são mostrados na Figura C.2:

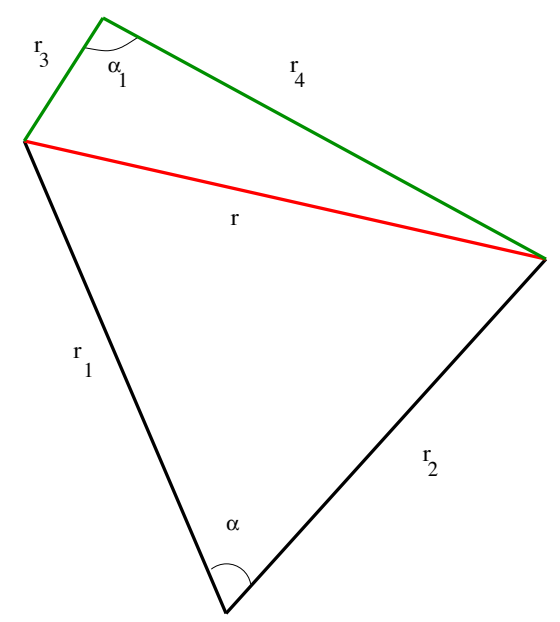

Fig. C.2 Representação geométrica dos elementos que aparecem no enunciado do Lema C.3.

\section{Demonstração:}

$$
\begin{aligned}
& \int_{0}^{\infty} d k k^{2} \frac{j_{l}\left(k r_{1}\right)}{\left(k r_{1}\right)^{m}} \frac{j_{m}\left(k r_{3}\right)}{\left(k r_{3}\right)^{m}} j_{l}\left(k r_{2}\right) j_{m}\left(k r_{4}\right)=\int_{0}^{\infty} d k k^{2} \frac{j_{m}\left(k r_{3}\right)}{\left(k r_{3}\right)^{m}} j_{m}\left(k r_{4}\right) \frac{1}{\left(k r_{1}\right)^{m}}\left(j_{l}\left(k r_{1}\right) j_{l}\left(k r_{2}\right)\right) \\
= & \frac{(-1)^{m}}{2} \int_{\left|r_{1}-r_{2}\right|}^{r_{1}+r_{2}} d r\left(\frac{r_{1} r_{2}}{r}\right)^{m-1} P_{l}^{-m}(\cos \alpha) \operatorname{sen}^{m} \alpha \frac{r^{m}}{r_{1}^{m}} \int_{0}^{\infty} d k k^{2} \frac{j_{m}\left(k r_{3}\right)}{\left(k r_{3}\right)^{m}} j_{m}\left(k r_{4}\right) j_{m}(k r) \\
= & \frac{(-1)^{m}}{2} \int_{\left|r_{1}-r_{2}\right|}^{r_{1}+r_{2}} d r\left(\frac{r_{1} r_{2}}{r}\right)^{m-1} P_{l}^{-m}(\cos \alpha) \operatorname{sen}^{m} \alpha \frac{r^{m}}{r_{1}^{m}} \frac{r^{m}}{r_{3}^{m}} \\
\times & \int_{0}^{\infty} d k k^{2} j_{m}\left(k r_{3}\right) j_{m}\left(k r_{4}\right) \frac{j_{m}(k r)}{(k r)^{m}} .
\end{aligned}
$$

Mas a última integral que aparece em (C.10) é justamente a calculada no Lema C.2 e portanto, 


$$
\begin{aligned}
& \int_{0}^{\infty} d k k^{2} \frac{j_{l}\left(k r_{1}\right)}{\left(k r_{1}\right)^{m}} \frac{j_{m}\left(k r_{3}\right)}{\left(k r_{3}\right)^{m}} j_{l}\left(k r_{2}\right) j_{m}\left(k r_{4}\right) \\
& =\frac{\pi}{8} \frac{1}{r_{1}^{m} r_{3}^{m}} \int_{\left|r_{1}-r_{2}\right|}^{r_{1}+r_{2}} d r \frac{\left(r_{1} r_{2} r_{3} r_{4}\right)^{m-1}}{r^{2 m}} P_{l}^{-m}(\cos \alpha) \operatorname{sen}^{m} \alpha P_{m}^{-m}\left(\cos \alpha_{1}\right) \operatorname{sen}^{m} \alpha_{1}
\end{aligned}
$$

com $r^{2}=r_{1}^{2}+r_{2}^{2}-2 r_{1} r_{2} \cos \alpha$ e $r^{2}=r_{3}^{2}+r_{4}^{2}-2 r_{3} r_{4} \cos \alpha_{1}$ como requerido.

Dos casos anteriores, nota-se que o procedimento para tratar o tipo de integral que nos interessa é transformar duas das funções de Bessel de ordem $l$ em uma função de Bessel de ordem $m$ e recair em um caso previamente resolvido para produtos de funções de Bessel de mesma ordem. Com o intuito de generalizar esse procedimento, consideremos o seguinte resultado:

Lema C.4 Sejam $j_{l}, j_{m}$ funções de Bessel esféricas, $l \leq m$. Para $r_{1}, r_{2}, \ldots, r_{n}, r \in$ $\mathbb{R}, m \in \mathbb{N}$, vale:

$$
\begin{aligned}
& \int_{0}^{\infty} d k k^{2} j_{m}(k r) \frac{j_{m}\left(k r_{1}\right)}{\left(k r_{1}\right)^{m}} \frac{j_{m}\left(k r_{2}\right)}{\left(k r_{2}\right)^{m}} \frac{j_{m}\left(k r_{3}\right)}{\left(k r_{3}\right)^{m}} \ldots \frac{j_{m}\left(k r_{n-1}\right)}{\left(k r_{n-1}\right)^{m}} j_{m}\left(k r_{n}\right) \\
& =\frac{\pi}{2^{n}} \frac{(-1)^{(n-1) m}}{r_{1}^{m} r_{2}^{m} r_{3}^{m} \ldots r_{n-1}^{m}} \int d \rho_{n-2} \ldots d \rho_{1} \frac{\left(r r_{1} r_{2} r_{3} \ldots r_{n}\right)^{m-1}}{r^{2 m}} P_{m}^{-m}\left(\cos \alpha_{1}\right) \operatorname{sen}^{m} \alpha_{1} \\
& \times P_{m}^{-m}\left(\cos \alpha_{2}\right) \operatorname{sen}^{m} \alpha_{2} \ldots P_{m}^{-m}\left(\cos \alpha_{n-1}\right) \operatorname{sen}^{m} \alpha_{n-1}
\end{aligned}
$$

para $n \geq 2$. $\alpha_{n-1}$ é o ângulo entre $r_{n}$ e $r_{n-1}$ e os $\alpha_{i}$ s sucessivos são sempre determinados como o ângulo entre $\rho_{i}$ e $r_{i}$, ou seja, $\rho_{n-2}^{2}=r_{n-1}^{2}+r_{n}^{2}-$ $2 r_{n-1} r_{n} \cos \alpha_{n-1}$ e $\rho_{i-1}^{2}=r_{i}^{2}+\rho_{i}^{2}-2 r_{i} \rho_{i} \cos \alpha_{i}$. Os elementos presentes na equação (C.11) são ilustrados na Figura C.3. 


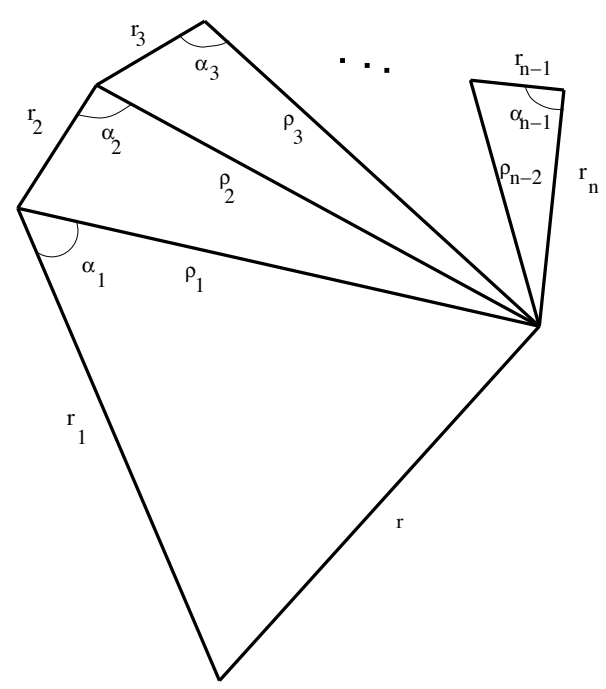

Fig. C.3 Representação geométrica dos elementos que aparecem no enunciado do Lema C.4.

\section{Demonstração:}

$$
\begin{aligned}
& \int_{0}^{\infty} d k k^{2} j_{m}(k r) \frac{j_{m}\left(k r_{1}\right)}{\left(k r_{1}\right)^{m}} \frac{j_{l}\left(k r_{2}\right)}{\left(k r_{2}\right)^{m}} \frac{j_{m}\left(k r_{3}\right)}{\left(k r_{3}\right)^{m}} \ldots \frac{j_{m}\left(k r_{n}\right)}{\left(k r_{n}\right)^{m}} j_{m}\left(k r_{n+1}\right) \\
& =\int_{0}^{\infty} d k \frac{(-1)^{m}}{2} \int d \rho_{(n-1)} \frac{1}{\left(k r_{n}\right)^{m}} k^{m}\left(\frac{r_{n} r_{(n+1)}}{\rho_{(n-1)}}\right)^{m-1} P_{m}^{-m}\left(\cos \alpha_{n}\right) \operatorname{sen}^{m} \alpha_{n} j_{m}\left(k \rho_{(n-1)}\right) \\
& \times k^{2} j_{m}(k r) \frac{j_{m}\left(k r_{1}\right)}{\left(k r_{1}\right)^{m}} \frac{j_{l}\left(k r_{2}\right)}{\left(k r_{2}\right)^{m}} \frac{j_{m}\left(k r_{3}\right)}{\left(k r_{3}\right)^{m}} \ldots \frac{j_{m}\left(k r_{(n-1)}\right)}{\left(k r_{(n-1)}\right)^{m}} j_{m}\left(k \rho_{(n-1)}\right) \\
& =\frac{(-1)^{m}}{2} \int d \rho_{(n-1)} \frac{1}{\left(r_{n}\right)^{m}}\left(\frac{r_{n} r_{(n+1)}}{\rho_{(n-1)}}\right)^{m-1} P_{m}^{-m}\left(\cos \alpha_{n}\right) \operatorname{sen}^{m} \alpha_{n} \\
& \times \int_{0}^{\infty} d k k^{2} j_{m}(k r) \frac{j_{m}\left(k r_{1}\right)}{\left(k r_{1}\right)^{m}} \frac{j_{m}\left(k r_{2}\right)}{\left(k r_{2}\right)^{m}} \ldots \frac{j_{m}\left(k r_{(n-1)}\right)}{\left(k r_{(n-1)}\right)^{m}} j_{m}\left(k \rho_{(n-1)}\right) .
\end{aligned}
$$

Mas esta é justamente a integral com a qual iniciamos, ou seja, (C.11) e portanto,

$$
\begin{aligned}
& \int_{0}^{\infty} d k k^{2} j_{m}(k r) \frac{j_{m}\left(k r_{1}\right)}{\left(k r_{1}\right)^{m}} \frac{j_{m}\left(k r_{2}\right)}{\left(k r_{2}\right)^{m}} \ldots \frac{j_{m}\left(k r_{n}\right)}{\left(k r_{n}\right)^{m}} j_{m}\left(k r_{n+1}\right) \\
& =\frac{\pi}{2^{n}} \frac{(-1)^{(n+2) m}}{r_{1}^{m} r_{2}^{m} r_{3}^{m} \ldots r_{n}^{m}} \int d \rho_{(n-1)} \ldots d \rho_{2} d \rho_{1} \frac{\left(r r_{1} r_{2} r_{3} \ldots r_{n+1}\right)^{m-1}}{r^{2 m}} \\
& \times P_{m}^{-m}\left(\cos \alpha_{1}\right) \operatorname{sen}^{m} \alpha_{1} P_{m}^{-m}\left(\cos \alpha_{2}\right) \operatorname{sen}^{m} \alpha_{2} \ldots P_{m}^{-m}\left(\cos \alpha_{n}\right) \operatorname{sen}^{m} \alpha_{n} .
\end{aligned}
$$


Como, pelo Lema C.2 o resultado vale para $n=2$, temos demonstrado o lema.

A sequência de operações necessárias à demonstração acima pode ser ilustrada como
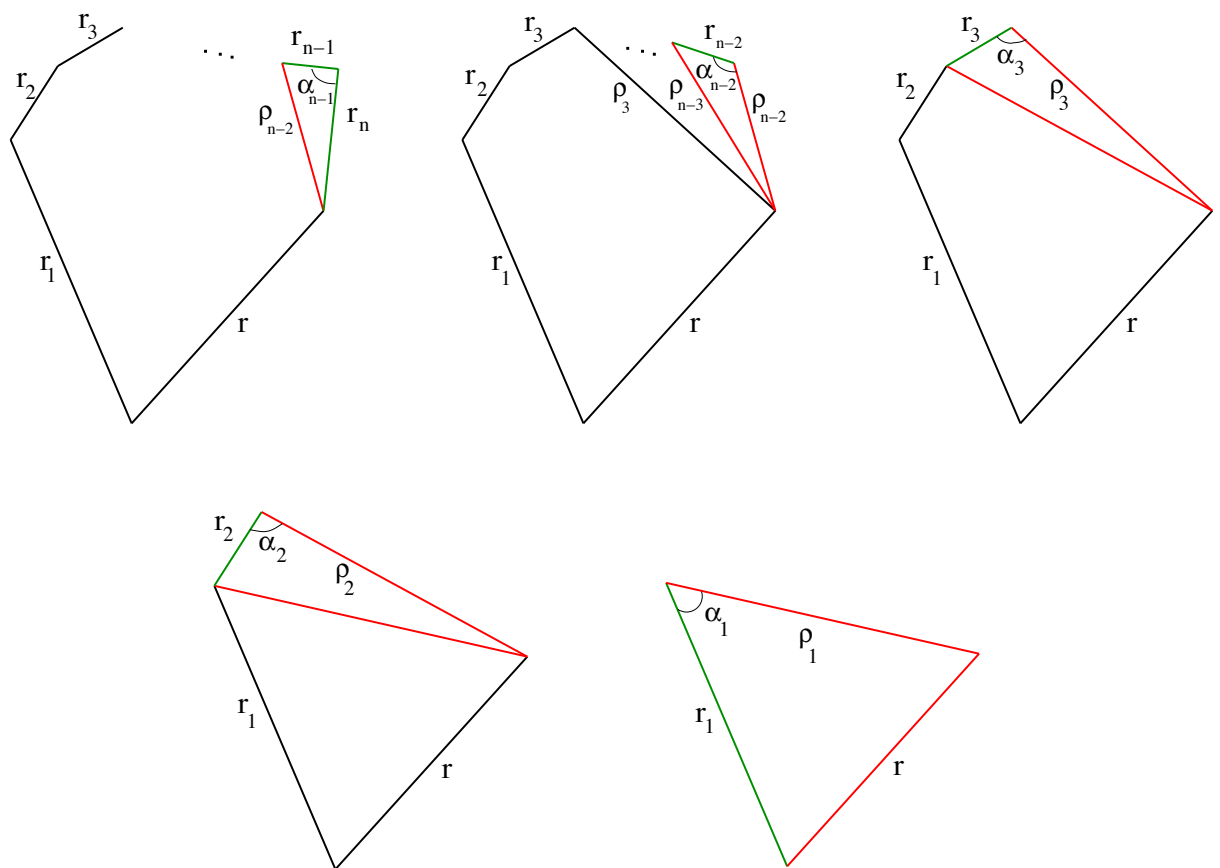

Fig. C.4 Processo de condensação dos trechos de vôo aleatório empregado no cálculo de (C.11). Sempre usamos o resultado do Lema C.1 para substituir dois trechos de vôo $-r_{n}$ e $r_{n-1}$, por exemplo - por uma diagonal $-\rho_{n-2}$ para o caso dos trechos destacados. O processo de redução a diagonais continua até que se fique apenas com um triângulo, que é calculado com o Lema C.2.

Caso tenhamos duas funções de Bessel de ordem diferente das demais, procedemos reunindo-as em uma função de Bessel apenas da mesma ordem que as demais e utilizando o resultado previamente demonstrado.

Proposição C.5 Sendo $j_{l}$ e $j_{m}$ funções de Bessel esféricas e $r_{1}, \ldots, r_{n} \in \mathbb{R}$ vale: 
138 Alguns resultados sobre funções de Bessel

$$
\begin{aligned}
& \int_{0}^{\infty} d k k^{2} j_{l}(k X) \frac{j_{l}(k R)}{(k R)^{m}} \frac{j_{m}\left(k r_{1}\right)}{\left(k r_{1}\right)^{m}} \frac{j_{m}\left(k r_{2}\right)}{\left(k r_{2}\right)^{m}} \ldots \frac{j_{m}\left(k r_{n-1}\right)}{\left(k r_{n-1}\right)^{m}} j_{m}\left(k r_{n}\right) \\
& =\frac{\pi}{2^{n+1}} \frac{(-1)^{n m}}{R^{m} r_{1}^{m} \ldots r_{n-1}^{m}} \int d \rho_{n-2} \ldots d \rho_{1} d r \frac{\left(R X r_{1} r_{2} \ldots r_{n}\right)^{m-1}}{r^{2 m}} P_{l}^{-m}(\cos \alpha) \operatorname{sen}^{m} \alpha \\
& \times P_{m}^{-m}\left(\cos \alpha_{1}\right) \operatorname{sen}^{m} \alpha_{1} P_{m}^{-m}\left(\cos \alpha_{2}\right) \operatorname{sen}^{m} \alpha_{2} \cdot P_{m}^{-m}\left(\cos \alpha_{(n-1)}\right) \operatorname{sen}^{m} \alpha_{(n-1)}
\end{aligned}
$$

para $n \geq 2$. Aqui $r^{2}=R^{2}+X^{2}-2 R X \cos \alpha, \alpha_{n-1}$ é o ângulo entre $r_{n}$ e $r_{n-1}$ e os $\alpha_{i}$ s sucessivos são sempre determinados como o ângulo entre $\rho_{i}$ e $r_{i}$, ou seja, $\rho_{n-2}^{2}=r_{n-1}^{2}+r_{n}^{2}-2 r_{n-1} r_{n} \cos \alpha_{n-1}$ e $\rho_{i-1}^{2}=r_{i}^{2}+\rho_{i}^{2}-2 r_{i} \rho_{i} \cos \alpha_{i}$.

A Figura C.5 representa geometricamente os elementos envolvidos na equação (C.14).

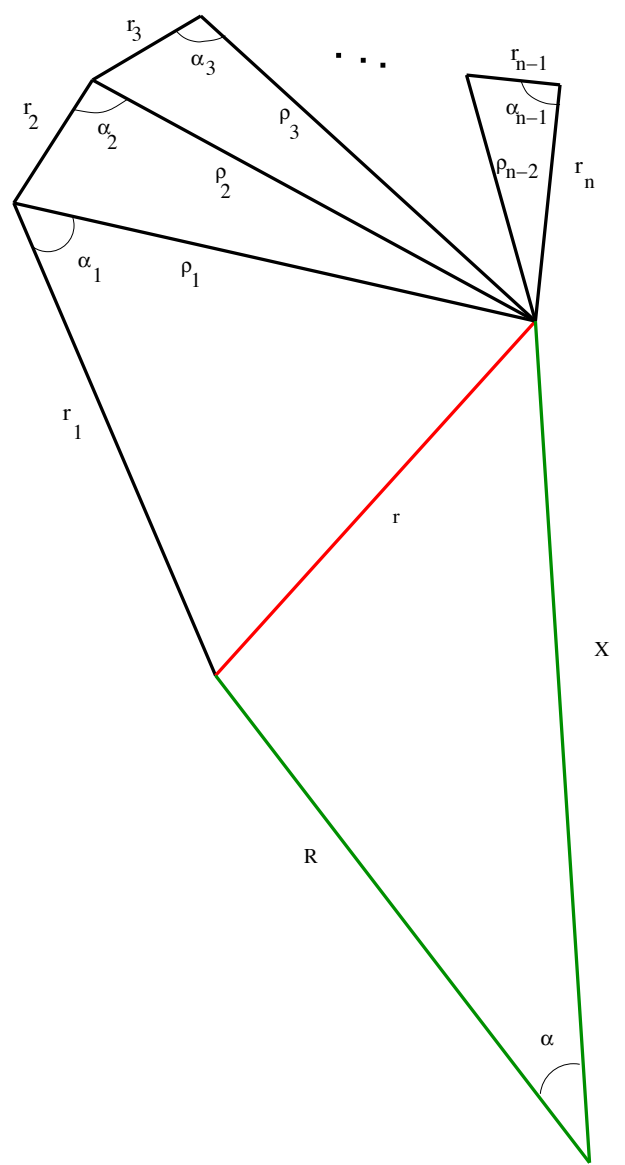

Fig. C.5 Representação geométrica dos elementos que aparecem no enunciado da Proposição C.5. 


\section{Demonstração:}

Fazendo uso do Lema C.1 podemos escrever, fazendo $r^{2}=R^{2}+X^{2}-$ $2 R X \cos \alpha$,

$$
\begin{aligned}
& \int_{0}^{\infty} d k k^{2} j_{l}(k X) \frac{j_{l}(k R)}{(k R)^{m}} \frac{j_{m}\left(k r_{1}\right)}{\left(k r_{1}\right)^{m}} \frac{j_{m}\left(k r_{2}\right)}{\left(k r_{2}\right)^{m}} \ldots \frac{j_{m}\left(k r_{n-1}\right)}{\left(k r_{n-1}\right)^{m}} j_{m}\left(k r_{n}\right) \\
& =\int_{0}^{\infty} d k k^{2} \frac{1}{(k R)^{m}} \frac{(-1)^{m}}{2} \int d r k^{m}\left(\frac{R X}{r}\right)^{m-1} P_{l}^{-m}(\cos \alpha) \operatorname{sen}^{m} \alpha \\
& \times j_{m}(k r) \frac{j_{m}\left(k r_{1}\right)}{\left(k r_{1}\right)^{m}} \frac{j_{m}\left(k r_{2}\right)}{\left(k r_{2}\right)^{m}} \ldots \frac{j_{m}\left(k r_{n-1}\right)}{\left(k r_{n-1}\right)^{m}} j_{m}\left(k r_{n}\right) \\
& =\frac{(-1)^{m}}{2} \frac{1}{R^{m}} \int d r\left(\frac{R X}{r}\right)^{m-1} P_{l}^{-m}(\cos \alpha) \operatorname{sen}^{m} \alpha \\
& \times \int_{0}^{\infty} d k k^{2} j_{m}(k r) \frac{j_{m}\left(k r_{1}\right)}{\left(k r_{1}\right)^{m}} \frac{j_{m}\left(k r_{2}\right)}{\left(k r_{2}\right)^{m}} \ldots \frac{j_{m}\left(k r_{n-1}\right)}{\left(k r_{n-1}\right)^{m}} j_{m}\left(k r_{n}\right) \\
& =\frac{\pi}{2^{(n+1)}} \frac{(-1)^{n m}}{R^{m} r_{1}^{m} r_{2}^{m} r_{3}^{m} \ldots r_{n-1}^{m}} \int d r \int d \rho_{n-2} \ldots d \rho_{1} \frac{\left(R X r_{1} r_{2} r_{3} \ldots r_{n}\right)^{m-1}}{r^{2 m}} \\
& \times P_{l}^{-m}(\cos \alpha) \operatorname{sen}^{m} \alpha P_{m}^{-m}\left(\cos \alpha_{1}\right) \operatorname{sen}^{m} \alpha_{1} \ldots P_{m}^{-m}\left(\cos \alpha_{(n-1)}\right) \operatorname{sen}^{m} \alpha_{(n-1)}
\end{aligned}
$$

onde, para estabelecer a última igualdade, utilizamos o Lema C.4.

\section{C.1.2 Conexão das integrais estudadas e o problema de vôos aleatórios}

Demonstraremos agora a possibilidade de escrever a integral que aparece em (C.14) da seguinte maneira:

Proposição C.6 Vale a identidade:

$$
\begin{aligned}
& \int_{0}^{\infty} d k k^{2} j_{m}(k r) \frac{j_{m}\left(k r_{1}\right)}{\left(k r_{1}\right)^{m}} \frac{j_{m}\left(k r_{2}\right)}{\left(k r_{2}\right)^{m}} \ldots \frac{j_{m}\left(k r_{n-1}\right)}{\left(k r_{n-1}\right)^{m}} j_{m}\left(k r_{n}\right)=\frac{\left(\frac{\pi}{2}\right)^{(n+1) / 2} 2^{m+1 / 2}}{(\Gamma(m+1+1 / 2))^{n-1}} \frac{r_{n}^{m}}{r^{m+2}} \times \\
& \frac{d}{d r}\left[(\Gamma(m+1+1 / 2))^{n-2} \int_{0}^{\infty} d k r\left(\frac{k r}{2}\right)^{m+1 / 2} J_{m+1+1 / 2}(k r) \prod_{i=1}^{n} \frac{J_{m+1 / 2}\left(k r_{i}\right)}{\left(k r_{i}\right)^{m+1 / 2}}\right] .
\end{aligned}
$$

\section{Demonstração:}




$$
\begin{aligned}
& \text { Alguns resultados sobre funções de Bessel } \\
& \int_{0}^{\infty} d k k^{2} j_{m}(k r) \frac{j_{m}\left(k r_{1}\right)}{\left(k r_{1}\right)^{m}} \frac{j_{m}\left(k r_{2}\right)}{\left(k r_{2}\right)^{m}} \ldots \frac{j_{m}\left(k r_{n-1}\right)}{\left(k r_{n-1}\right)^{m}} j_{m}\left(k r_{n}\right) \\
& =\int_{0}^{\infty} d k k^{2}\left(\frac{\pi}{2}\right)^{(n+1) / 2} \frac{J_{m+1 / 2}(k r) J_{m+1 / 2}\left(k r_{1}\right) \ldots J_{m+1 / 2}\left(k r_{n}\right)}{\sqrt{k r}\left(\sqrt{k r_{1}} \ldots \sqrt{k r_{n}}\right)\left(k r_{1}\right)^{m}\left(k r_{2}\right)^{m} \ldots\left(k r_{n-1}\right)^{m}} \\
& =\left(\frac{\pi}{2}\right)^{(n+1) / 2} \int_{0}^{\infty} d k k^{2} \frac{k^{m}}{\sqrt{k}} \frac{r_{n}^{m}}{\sqrt{r}} \frac{1}{k^{m+2}} \frac{(k r)^{m+2}}{r^{m+2}} J_{\frac{2 m+1}{2}}(k r) \prod_{i=1}^{\frac{J_{2 m+1}}{2}} \frac{\left(k r_{i}\right)}{\left(k r_{i}\right)^{\frac{2 m+1}{2}}} \\
& =\left(\frac{\pi}{2}\right)^{(n+1) / 2} \int_{0}^{\infty} d k \frac{r_{n}^{m}}{r^{m+2}} \frac{1}{k} \frac{d}{d r}\left((k r)^{m+1+1 / 2} J_{m+1+1 / 2}(k r)\right) \prod_{i=1}^{n} \frac{J_{\frac{2 m+1}{2}}\left(k r_{i}\right)}{\left(k r_{i}\right)^{\frac{2 m+1}{2}}}
\end{aligned}
$$

pois

$$
(k r)^{m+1+1 / 2} J_{\frac{2 m+1}{2}}(k r)=\frac{1}{k} \frac{d}{d r}\left((k r)^{m+1+1 / 2} J_{m+1+1 / 2}(k r)\right)
$$

e

$$
j_{n}(x)=\sqrt{\frac{\pi}{2 x}} J_{n+1 / 2}(x) .
$$

Rearranjando a última integral em (C.16) obtemos:

$$
\left(\frac{\pi}{2}\right)^{(n+1) / 2} 2^{m+1 / 2} \frac{r_{n}^{m}}{r^{m+2}} \frac{d}{d r}\left(\int_{0}^{\infty} d k r\left(\frac{k r}{2}\right)^{m+1 / 2} J_{m+1+1 / 2}(k r) \prod_{i=1}^{n} \frac{J_{\frac{2 m+1}{2}}\left(k r_{i}\right)}{\left(k r_{i}\right)^{\frac{2 m+1}{2}}}\right) .
$$

de onde obtém-se a expressão do enunciado da proposição.

Antecipando o significado que se atribuirá a esta expressão no que segue, denotaremos:

$$
\begin{aligned}
& P_{n}\left(r ; r_{1}, r_{2}, \ldots, r_{n} \mid 2 m+3\right):=(\Gamma(m+1+1 / 2))^{n-1} \times \\
& \int_{0}^{\infty} d k r\left(\frac{k r}{2}\right)^{m+1 / 2} J_{m+1+1 / 2}(k r) \prod_{i=1}^{n} \frac{J_{m+1 / 2}\left(k r_{i}\right)}{\left(k r_{i}\right)^{m+1 / 2}} .
\end{aligned}
$$

Empregando esta notação escrevemos o resultado da integral de $n$ funções de Bessel esféricas que estamos estudando da seguinte forma:

$$
\begin{aligned}
& \int_{0}^{\infty} d k k^{2} j_{m}(k r) \frac{j_{m}\left(k r_{1}\right)}{\left(k r_{1}\right)^{m}} \frac{j_{m}\left(k r_{3}\right)}{\left(k r_{3}\right)^{m}} \ldots \frac{j_{m}\left(k r_{n-1}\right)}{\left(k r_{n-1}\right)^{m}} j_{m}\left(k r_{n}\right)= \\
& \frac{\left(\frac{\pi}{2}\right)^{(n+1) / 2} 2^{m+1 / 2}}{(\Gamma(m+1+1 / 2))^{n-1}} \frac{r_{n}^{m}}{r^{m+2}} \frac{d}{d r} P_{n}\left(r ; r_{1}, r_{2}, \ldots, r_{n} \mid 2 m+3\right) .
\end{aligned}
$$


Com efeito, aprendemos em [53], seção 13.48, estudando o problema de vôos aleatórios em $D$ dimensões ${ }^{1}$, que a probabilidade de que após percorrer $n$ trechos de comprimentos $r_{1}, r_{2}, \ldots, r_{n}$ (sempre com velocidade constante) um caminhante esteja a uma distância menor ou igual a $r$ do ponto inicial do movimento é justamente $P_{n}\left(r ; r_{1}, r_{2}, \ldots, r_{n} \mid D\right)$. Além disso a quantidade:

$$
p_{n}\left(r ; r_{1}, r_{2}, \ldots, r_{n} \mid D\right):=\frac{d P_{n}\left(r ; r_{1}, r_{2}, \ldots, r_{n} \mid D\right)}{d r}
$$

é a densidade de probabilidade de que um caminhante executando um vôo aleatório de passos $r_{1}, r_{2}, \ldots, r_{n}$ em um espaço de dimensão $D$ esteja a uma distância $r$ da origem de seu movimento. Vale notar que caso $r$ seja superior à soma $r_{1}+r_{2}+\ldots+r_{n}$ a densidade de probabilidade apresentada anula-se identicamente.

A referência [53] apresenta ainda seguinte expressão para $P_{n}\left(r ; r_{1}, r_{2}, \ldots, r_{n} \mid D\right)$ :

$$
\begin{aligned}
P_{n}\left(r ; r_{1}, r_{2}, \ldots, r_{n} \mid D\right)= & \left(\frac{\Gamma(D / 2)}{\Gamma(D / 2-1 / 2) \Gamma(1 / 2)}\right)^{n-1} \int_{0}^{\pi} \int_{0}^{\pi} \ldots \int_{0}^{\pi} \\
& \int \prod_{m=1}^{n-1} \operatorname{sen}^{D-2}\left(\theta_{m}\right) d \theta_{m} .
\end{aligned}
$$

A integração em $\theta_{n-1}$ estende-se a todos os valores desta variável que fazem com que $s_{n}<r$, com $s_{n}^{2}=s_{n-1}^{2}+r_{n}^{2}-2 s_{n-1} r_{n} \cos \theta_{n-1}$. Representando em uma figura o vôo aleatório de três passos, teríamos o esquema apresentado na Figura C.6.

${ }^{1}$ Para uma abordagem histórica e detalhada do problema de vôos aleatórios e evolução em sua abordagem, ver [55]. 


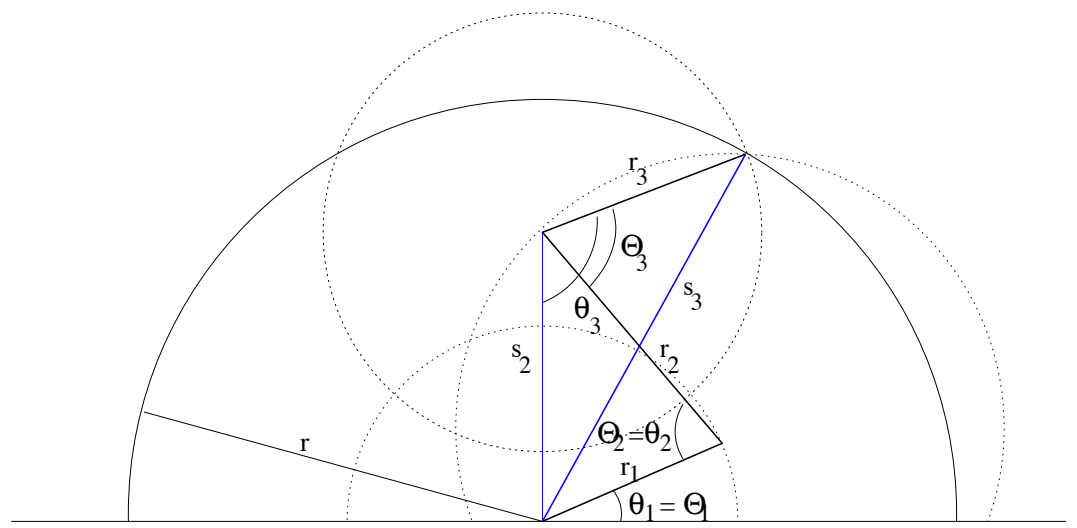

Fig. C.6 Representação pictórica de um vôo aleatório com três passos. Representam-se os elementos presentes na equação (C.20). Os ângulos $\Theta_{1}, \Theta_{2}, \ldots, \Theta_{n}$ são aqueles formados entre dois segmentos sucessivos do movimento. Os ângulos $\theta_{1}, \theta_{2}, \ldots, \theta_{n}$ são formados entre os lados $s_{i-1}$ e $r_{i}$.

Para verificar que a fórmula que obtivemos para a probabilidade é equivalente a essa caracterização notamos que, pelas fórmulas de Rodrigues para polinômios de Legendre associados:

$$
P_{l}^{m}(z)=\frac{1}{2^{l} l !}\left(1-z^{2}\right)^{m / 2} \frac{d^{l+m}}{d z^{l+m}}\left(\left(z^{2}-1\right)^{l}\right)
$$

de onde segue que:

$$
P_{m}^{-m}(\cos \theta)=\frac{(-1)^{m}}{2^{m} m !} \operatorname{sen}^{m} \theta
$$

Inserindo esta expressão em (C.14) verifica-se a igualdade requerida através da expressão (C.18).

Notamos ainda que formas explícitas para as distribuições de probabilidade de vôos aleatórios em quaisquer dimensões são problemas para os quais não se conhecem respostas, embora esforços nesse sentido sejam feitos, por exemplo, em [61], [62].

Reuniremos agora alguns dos resultados apresentados para escrevê-los de maneira mais sintética. Especificamente, das Proposições C.5 e C.16 segue diretamente que:

Teorema C.7 Vale a igualdade: 


$$
\begin{aligned}
& \int_{0}^{\infty} d k k^{2} j_{l}(k X) \frac{j_{l}(k R)}{(k R)^{m}} \frac{j_{m}\left(k r_{1}\right)}{\left(k r_{1}\right)^{m}} \frac{j_{m}\left(k r_{2}\right)}{\left(k r_{2}\right)^{m}} \ldots \frac{j_{m}\left(k r_{n-1}\right)}{\left(k r_{n-1}\right)^{m}} j_{m}\left(k r_{n}\right)= \\
& \frac{(-1)^{m}}{2} \frac{\left(\frac{\pi}{2}\right)^{(n+1) / 2}}{(\Gamma(m+3 / 2))^{n-1}} \int d(\cos \alpha) \frac{1}{r^{2}}\left(\frac{X r_{n}}{r^{2}}\right)^{m} P_{l}^{-m}(\cos \alpha) \operatorname{sen}^{m} \alpha \\
& \times p_{n}\left(r ; r_{1}, \ldots, r_{n} \mid 2 m+3\right)
\end{aligned}
$$

com $r^{2}=R^{2}+X^{2}-2 R X \cos \alpha, P_{l}^{-m}(\cos \alpha)$ um polinômio de Legendre associado e $p_{n}\left(r ; r_{1}, \ldots, r_{n} \mid 2 m+3\right)$ a densidade de probabilidade de que após $n$ passos de vôo aleatório de comprimentos $r_{1}, r_{2}, \ldots, r_{n}$ em $2 m+3$ dimensões o caminhante esteja a uma distância $r$ da origem do movimento.

\section{C.2 Segundo grupo de resultados: Ampliação da família de integrais que podem ser relacionadas a vôos aleatórios}

Estudaremos nesta Seção a possibilidade de expressar um conjunto maior de integrais na forma de probabilidades associadas a vôos aleatórios. Especificamente, partiremos de expressões da forma estudada:

$$
H_{l m}\left(R, X, r_{1}, \ldots, r_{n}\right)=\int_{0}^{\infty} d k k^{2} j_{l}(k X) \frac{j_{l}(k R)}{(k R)^{m}} \frac{j_{m}\left(k r_{1}\right)}{\left(k r_{1}\right)^{m}} \frac{j_{m}\left(k r_{2}\right)}{\left(k r_{2}\right)^{m}} \ldots \frac{j_{m}\left(k r_{n-1}\right)}{\left(k r_{n-1}\right)^{m}} j_{m}\left(k r_{n}\right)
$$

para avaliar se há maneiras de expressar integrais da forma:

$G_{l m}\left(R, X, r_{1}, \ldots, r_{n}\right)=\int_{0}^{\infty} d k k^{2} f(k) j_{l}(k X) \frac{j_{l}(k R)}{(k R)^{m}} \frac{j_{m}\left(k r_{1}\right)}{\left(k r_{1}\right)^{m}} \frac{j_{m}\left(k r_{2}\right)}{\left(k r_{2}\right)^{m}} \ldots \frac{j_{m}\left(k r_{n-1}\right)}{\left(k r_{n-1}\right)^{m}} j_{m}\left(k r_{n}\right)$

em termos de combinações de $H_{l^{\prime} m^{\prime}}\left(R, X, r_{1}, \ldots, r_{n}\right)$ 's para $l^{\prime}$ 's e $m^{\prime}$ s adequados. Em $G_{l m}, f(k)$ é uma função tal que, em sua série de Taylor, apenas termos com potências pares de $k$ apareçam. Naturalmente a função $f(k)$ mais simples possível é do tipo $a k^{0}$, para qualquer constante $a$ e portanto a resposta sobre a possibilidade investigada é óbvia. Estudaremos com detalhe o segundo monômio da expansão, a saber, tomaremos $f(k)=k^{2}$.

\section{C.2.1 Estudo de $G_{l m}\left(R, X, r_{1}, \ldots, r_{n}\right)$ com $f(k)=k^{2}$}

Tomemos o seguinte objeto como nosso tema de estudo:

$G_{l m}^{(2)}\left(R, X, r_{1}, \ldots, r_{n}\right)=\int_{0}^{\infty} d k k^{2}\left(k^{2}\right) j_{l}(k X) \frac{j_{l}(k R)}{(k R)^{m}} \frac{j_{m}\left(k r_{1}\right)}{\left(k r_{1}\right)^{m}} \frac{j_{m}\left(k r_{2}\right)}{\left(k r_{2}\right)^{m}} \ldots \frac{j_{m}\left(k r_{n-1}\right)}{\left(k r_{n-1}\right)^{m}} j_{m}\left(k r_{n}\right)$

o número dois sobrescrito indica a potência de $k$ na expansão de $f(k)$ empregada. 
144 Alguns resultados sobre funções de Bessel

Lembremo-nos inicialmente uma das relações de recorrência satisfeitas pelas funções de Bessel esféricas ${ }^{2}$ :

$$
\frac{d}{d x}\left(x^{\nu+1} j_{\nu}(x)\right)=x^{\nu+1} j_{\nu-1}(x) .
$$

Segue, portanto, para uma ordem inteira $l$ que:

$$
x^{l+2} j_{l}(x)=\frac{d}{d x}\left(x^{l+2} j_{l+1}(x)\right) .
$$

Pela regra da cadeia, temos também:

$$
\frac{d}{d x}\left[y(x)^{l+2} j_{l+1}(y(x))\right]=\frac{d}{d y}\left[y(x)^{l+2} j_{l+1}(y(x))\right] \frac{d y}{d x} .
$$

Se $y(x)=k x$,

$$
j_{l}(k x)=\frac{1}{k} \frac{1}{(k x)^{l+2}} \frac{d}{d x}\left[(k x)^{l+2} j_{l+1}(k x)\right] .
$$

Inserindo (C.26) em (C.24) temos:

$$
\begin{aligned}
G_{l m}^{(2)}\left(R, X, r_{1}, \ldots, r_{n}\right)= & \int_{0}^{\infty} d k k^{2}\left(k^{2}\right) \frac{1}{(k R)^{m}} \frac{1}{k} \frac{1}{(k R)^{l+2}} \frac{d}{d R}\left[(k R)^{l+2} j_{l+1}(k R)\right] \\
& \times \frac{1}{k} \frac{1}{(k X)^{l+2}} \frac{d}{d X}\left[(k X)^{l+2} j_{l+1}(k X)\right] \\
& \times \frac{j_{m}\left(k r_{1}\right)}{\left(k r_{1}\right)^{m}} \frac{j_{m}\left(k r_{2}\right)}{\left(k r_{2}\right)^{m}} \ldots \frac{j_{m}\left(k r_{n-1}\right)}{\left(k r_{n-1}\right)^{m}} j_{m}\left(k r_{n}\right) \\
= & \frac{1}{(R X)^{l+2}} \int_{0}^{\infty} d k k^{2} \frac{1}{(k R)^{m}} \frac{d}{d R}\left[R^{l+2} j_{l+1}(k R)\right] \\
& \times \frac{d}{d X}\left[(X)^{l+2} j_{l+1}(k X)\right] \frac{j_{m}\left(k r_{1}\right)}{\left(k r_{1}\right)^{m}} \frac{j_{m}\left(k r_{2}\right)}{\left(k r_{2}\right)^{m}} \ldots \frac{j_{m}\left(k r_{n-1}\right)}{\left(k r_{n-1}\right)^{m}} j_{m}\left(k r_{n}\right) .
\end{aligned}
$$

Mas,

$$
\frac{1}{(k R)^{m}} \frac{d}{d R} u(R)=\frac{d}{d R}\left[\frac{u(R)}{(k R)^{m}}\right]+\frac{m}{R} \frac{u(R)}{(k R)^{m}}
$$

${ }^{2}$ Essa identidade segue da conhecida relação de recorrência para funções de Bessel:

$$
\frac{d}{d x}\left(x^{\nu} J_{\nu}(x)\right)=x^{\nu} J_{\nu-1}(x),
$$

e do fato que $j_{\nu}=\sqrt{\frac{\pi}{2 x}} J_{\nu+1 / 2}(x)$. 

para uma função $u(R)$ diferenciável. Logo,

$$
\begin{aligned}
G_{l m}^{(2)}\left(R, X, r_{1}, \ldots, r_{n}\right)= & \frac{1}{(R X)^{l+2}} \frac{d}{d X}\left\{\frac { d } { d R } \left[( R X ) ^ { l + 2 } \left(\int_{0}^{\infty} d k k^{2} \frac{j_{l+1}(k R)}{(k R)^{m}} j_{l+1}(k X)\right.\right.\right. \\
& \left.\left.\times \frac{j_{m}\left(k r_{1}\right)}{\left(k r_{1}\right)^{m}} \frac{j_{m}\left(k r_{2}\right)}{\left(k r_{2}\right)^{m}} \ldots \frac{j_{m}\left(k r_{n-1}\right)}{\left(k r_{n-1}\right)^{m}} j_{m}\left(k r_{n}\right)\right)\right] \\
& +\frac{m}{R}(R X)^{l+2}\left(\int_{0}^{\infty} d k k^{2} \frac{j_{l+1}(k R)}{(k R)^{m}} j_{l+1}(k X)\right. \\
& \left.\left.\times \frac{j_{m}\left(k r_{1}\right)}{\left(k r_{1}\right)^{m}} \frac{j_{m}\left(k r_{2}\right)}{\left(k r_{2}\right)^{m}} \ldots \frac{j_{m}\left(k r_{n-1}\right)}{\left(k r_{n-1}\right)^{m}} j_{m}\left(k r_{n}\right)\right)\right\} .
\end{aligned}
$$

Obtivemos, então a seguinte relação:

$G_{l m}^{(2)}\left(R, X, r_{1}, \ldots, r_{n}\right)=\frac{1}{(R X)^{l+2}} \frac{\partial}{\partial X}\left(\frac{\partial}{\partial R}+\frac{m}{R}\right)\left[(R X)^{l+2} H_{(l+1) m}\left(R, X, r_{1}, \ldots, r_{n}\right)\right]$,

ou seja, $G_{l m}^{(2)}\left(R, X, r_{1}, \ldots, r_{n}\right)$ pode ser expressa como uma combinação de derivadas de $H_{(l+1) m}\left(R, X, r_{1}, \ldots, r_{n}\right)$ e era este o resultado que buscávamos obter.

\section{C.2.2 Indicação de que o argumento vale para potências mais altas na expansão de $f(k)$}

Não obteremos uma expressão fechada pra maneiras de expressar $G_{l m}^{(q)}\left(R, X, r_{1}, \ldots, r_{n}\right)$ para $q$ 's pares quaisquer, mas indicaremos como o argumento que acabamos de apresentar pode ser generalizado quando conveniente.

Inicialmente notamos que potências mais altas na expansão de $f(k)$ terão que ser canceladas por fatores correspondentes de $1 / k$ obtidos através da equação (C.26) iterada um número de vezes suficiente. Se nos interessássemos pelo monômio de quarto grau na expansão de $f(k)$, escreveríamos na forma

$$
\begin{aligned}
j_{l}(k x) & =\frac{1}{k} \frac{1}{x^{l+2}} \frac{d}{d x}\left[x^{l+2}\left(\frac{1}{k} \frac{1}{x^{l+3}} \frac{d}{d x}\left[x^{l+3} j_{l+2}(k x)\right]\right)\right] \\
& =\frac{1}{k^{2}}\left[\frac{1}{x^{l+3}} \frac{d^{2}}{d x^{2}}\left(x^{l+3} j_{l+2}(k x)\right)-\frac{1}{x^{l+4}} \frac{d}{d x}\left(x^{l+3} j_{l+2}(k x)\right)\right],
\end{aligned}
$$

tanto para $j_{l}(k R)$ quanto para $j_{l}(k X)$. Temos que lembrar que nas equações originais aparece $\frac{j_{l}(k R)}{(k R)^{m}}$, e portanto teremos que intercambiar derivadas de um determinado grau em relação a $\mathrm{R}$ com um termo da forma $1 /(k R)^{m}$. Isso, entretanto, pode ser facilmente conseguido através da fórmula de Leibnitz para derivadas n-ésimas de produtos de funções: 


$$
\begin{aligned}
\frac{1}{(k R)^{m}} \frac{d^{q}}{d R^{q}}[u(R)]= & \frac{d^{q}}{d R^{q}}\left(\frac{u(R)}{(k R)^{m}}\right) \\
& -\sum_{r=0}^{q-1}\left(\begin{array}{c}
q-1 \\
r
\end{array}\right) \frac{d^{r}}{d R^{r}}[u(R)] \frac{(-1)^{q-r}}{(k R)^{m}} \frac{m(m-1) \ldots(m-q-r)}{R^{q-r}} .
\end{aligned}
$$

Esse processo pode ser iterado até que tenham sido intercambiadas todas as derivadas com relação a $\mathrm{R}$ e os termos $1 /(k R)^{m}$.

Vemos, portanto, que embora o trabalho analítico seja grande, as $G_{l m}^{(q)}\left(R, X, r_{1}, \ldots, r_{n}\right) \mathrm{s}$ sempre podem ser obtidas, para $q$ par, em termos de derivadas de $H_{(l+q / 2) m}\left(R, X, r_{1}, \ldots, r_{n}\right)$.

\section{C.3 Terceiro grupo de resultados: séries de Fourier-Bessel}

Mostraremos aqui uma maneira alternativa de expressar a integral de produtos de funções de Bessel que nos interessa utilizando a série de Fourier-Bessel. Este procedimento é possível pois a distribuição de probabilidade a que nossas integrais estão associadas é identicamente nula caso a distância entre o ponto inicial do vôo aleatório e seu ponto final seja superior à soma de todos os trechos intermediários da trajetória real.

\section{C.3.1 Séries de Fourier-Bessel das distribuições de probabilidade}

Vamos tomar uma função $f(r), 0 \leq r \leq a$, que possa ser expandida como:

$$
f(r)=\sum_{n=1}^{\infty} A_{l n} j_{l}\left(x_{l n} r\right)
$$

com coeficientes dados por:

$$
A_{l n}=\frac{2}{a^{3} j_{l+1}^{2}\left(x_{l n} a\right)} \int_{0}^{a} d r r^{2} j_{l}\left(x_{l n} r\right) f(r)
$$

onde

$$
x_{l n}:=\frac{\alpha_{n}^{l}}{a}
$$

e $\alpha_{n}^{l}$ é o n-ésimo zero da função de Bessel esférica de ordem $l$. Esta expansão é chamada de série de Fourier-Bessel [64].

Suponha que desejemos determinar os coeficientes da expansão em série de Fourier-Bessel da seguinte função:

$$
f_{m, n}(r)=\int_{0}^{\infty} d k k^{2} j_{m}(k r) \frac{j_{m}\left(k r_{1}\right)}{\left(k r_{1}\right)^{m}} \frac{j_{m}\left(k r_{2}\right)}{\left(k r_{2}\right)^{m}} \ldots \frac{j_{m}\left(k r_{n-1}\right)}{\left(k r_{n-1}\right)^{m}} j_{m}\left(k r_{n}\right)
$$


com $0 \leq r \leq r_{1}+r_{2}+\ldots+r_{n}{ }^{3}$. Chamaremos, neste caso, $a=r_{1}+r_{2}+\ldots+r_{n}$. O coeficiente da expansão será, portanto,

$$
\begin{aligned}
A_{m q}= & \frac{2}{a^{3} j_{m+1}^{2}\left(x_{m q} a\right)} \int_{0}^{a} d r j_{m}\left(x_{m q} r\right) f_{m, n}(r) \\
= & \int_{0}^{\infty} d k k^{2} \frac{j_{m}\left(k r_{1}\right)}{\left(k r_{1}\right)^{m}} \frac{j_{m}\left(k r_{2}\right)}{\left(k r_{2}\right)^{m}} \cdots \frac{j_{m}\left(k r_{n-1}\right)}{\left(k r_{n-1}\right)^{m}} j_{m}\left(k r_{n}\right) \\
& \times \underbrace{\int_{0}^{\infty} d r r^{2} j_{m}\left(x_{m q} r\right) j_{m}(k r)}_{\frac{\pi}{2\left(x_{m q}\right)^{2}} \delta\left(k-x_{m q}\right)} .
\end{aligned}
$$

A integral em $r$ foi prolongada a infinito justamente porque a função $f(r)$ anula-se quando $r>a$, e portanto a alteração no domínio de integração não tem efeito outro que permitir o uso da relação de ortogonalidade das funções de Bessel. Como resultado escreve-se:

$f_{m, n}(r)=\sum_{q=1}^{\infty} \frac{\pi}{a^{3} j_{m+1}^{2}\left(x_{m q} a\right)} j_{m}\left(x_{m q} r\right) \frac{j_{m}\left(x_{m q} r_{1}\right)}{\left(x_{m q} r_{1}\right)^{m}} \frac{j_{m}\left(x_{m q} r_{2}\right)}{\left(x_{m q} r_{2}\right)^{m}} \ldots \frac{j_{m}\left(x_{m q} r_{n-1}\right)}{\left(x_{m q} r_{n-1}\right)^{m}} j_{m}\left(x_{m q} r_{n}\right)$.

Este é o resultado da expansão de $f_{m, n}(r)$ em uma série de Fourier-Bessel.

Notemos que dispomos de duas maneiras de expressar $f(r)$ : através das integrais que foram apresentadas em (C.14) para o caso particular em que $l=m$ e através de (C.33). Verificaremos para os primeiros casos particulares que as duas expressões coincidem:

Casos particulares.

- $n=1$

Neste caso (C.33) dá:

$$
f_{m, 1}(r)=\frac{\pi}{a^{3}} \sum_{q=1}^{\infty} \frac{j_{m}\left(x_{m q} r\right) j_{m}\left(x_{m q} r_{1}\right)}{j_{m+1}^{2}\left(x_{m q} a\right)}=\frac{\pi}{2} \frac{1}{r_{1}^{2}} \delta\left(r-r_{1}\right)
$$

que tem justamente a forma da distribuição de probabilidades encontrada quando se permite apenas um trecho de vôo.

- $n=2$

Novamente por (C.33):

${ }^{3}$ Notemos que, com efeito, que $f_{m, n}(r)$ anula-se caso $r$ seja maior que $r_{1}+r_{2}+\ldots+r_{n}$ por causa de (C.18). 


$$
\begin{aligned}
f_{m, 2}(r)= & \frac{\pi}{a^{3}} \sum_{q=1}^{\infty} \frac{1}{j_{m+1}^{2}\left(x_{m q} a\right)} \frac{j_{m}\left(x_{m q} r_{1}\right)}{\left(x_{m q} r_{1}\right)^{m}} j_{m}\left(x_{m q} r\right) j_{m}\left(x_{m q} r_{2}\right) \\
= & \frac{\pi}{a^{3}} \sum_{q=1}^{\infty} \frac{1}{j_{m+1}^{2}\left(x_{m q} a\right)} \frac{j_{m}\left(x_{m q} r_{1}\right)}{\left(x_{m q} r_{1}\right)^{m}} \\
& \times\left[\frac{(-1)^{m}}{2} \int d \rho\left(x_{m q}\right)^{m}\left(\frac{r r_{2}}{\rho}\right)^{m-1} P_{m}^{-m}\left(\cos \alpha_{1}\right) \operatorname{sen}^{m} \alpha_{1} j_{m}\left(x_{m q} \rho\right)\right] \\
= & \frac{\pi}{a^{3}} \frac{(-1)^{m}}{2} \int d \rho \frac{1}{r_{1}}\left(\frac{r r_{2}}{r_{1} \rho}\right)^{m-1} P_{m}^{-m}\left(\cos \alpha_{1}\right) \operatorname{sen}^{m} \alpha_{1} \sum_{q=1}^{\infty} \frac{j_{m}\left(x_{m q} r_{1}\right) j_{m}\left(x_{m q} \rho\right)}{j_{m+1}^{2}\left(x_{m q} a\right)} \\
= & (-1)^{m} \frac{\pi}{4} \frac{\left(r r_{2}\right)^{m-1}}{r_{1}^{2 m+1}} P_{m}^{-m}\left(\cos \alpha_{1}\right) \operatorname{sen}^{m} \alpha_{1} .
\end{aligned}
$$

Ou seja, obtivemos exatamente o resultado mostrado no Lema C.2 para o caso em que $l=m$.

O mesmo procedimento realizado no caso em que $n=2$ pode ser repetido para mostrar que, ordem a ordem, há a necessária igualdade entre as duas maneiras de expressar $f_{m, n}(r)$.

\section{C.4 Quarto grupo de resultados: uma relação tipo-completeza para funções de Bessel esféricas}

Os resultados apresentados nesta seção deram origem a [6].

\section{C.4.1 Motivação: Um problema de cosmologia}

Em cosmologia, no contexto dos estudos de radiação cósmica de fundo integrais envolvendo produtos de funções de Bessel da seguinte forma aparecem recorrentemente [4]:

$$
\int_{0}^{\infty} d k k^{2} \frac{j_{l}(k R)}{(k R)^{2}} j_{l}(k X) \frac{j_{2}\left(k r_{1}\right)}{\left(k r_{1}\right)^{2}} \ldots \frac{j_{2}\left(k r_{n-1}\right)}{\left(k r_{n-1}\right)^{2}} j_{2}\left(k r_{n}\right) .
$$

Aplicando o resultado do Lema C.1 a esta integral obtemos: 


$$
\begin{aligned}
& \int_{0}^{\infty} d k k^{2} \frac{j_{l}(k R)}{(k R)^{2}} j_{l}(k X) \frac{j_{2}\left(k r_{1}\right)}{\left(k r_{1}\right)^{2}} \frac{j_{2}\left(k r_{2}\right)}{\left(k r_{2}\right)^{2}} \ldots \frac{j_{2}\left(k r_{n-1}\right)}{\left(k r_{n-1}\right)^{2}} j_{2}\left(k r_{n}\right) \\
& =\frac{1}{2} \int_{-1}^{1} d(\cos \alpha) \frac{1}{R^{2}}\left(\frac{R X}{r}\right)^{2} P_{l}^{-2}(\cos \alpha) \operatorname{sen}^{2} \alpha \\
& \times \int_{0}^{\infty} d k k^{2} j_{2}(k r) \frac{j_{2}\left(k r_{1}\right)}{\left(k r_{1}\right)^{2}} \frac{j_{2}\left(k r_{2}\right)}{\left(k r_{2}\right)^{2}} \ldots \frac{j_{2}\left(k r_{n-1}\right)}{\left(k r_{n-1}\right)^{2}} j_{2}\left(k r_{n}\right) \\
& =\frac{1}{2} \frac{\left(\frac{\pi}{2}\right)^{(n+1) / 2}}{(\Gamma(7 / 2))^{n-1}} \int_{-1}^{1} d(\cos \alpha) P_{l}^{-2}(\cos \alpha) \operatorname{sen}^{2} \alpha\left(\frac{X r_{n}}{r^{3}}\right)^{2} \\
& \times \frac{d}{d r} P_{n}\left(r ; r_{1}, r_{2}, \ldots, r_{n} \mid 7\right)
\end{aligned}
$$

com $r^{2}=R^{2}+X^{2}-2 R X \cos \alpha$. Portanto, podemos expressar (C.36) como uma integral envolvendo certas funções das distâncias envolvidas no problema (ainda que na forma de ângulos) e uma distribuição de probabilidades. Como a densidade de probabilidades anula-se identicamente caso $r>r_{1}+r_{2}+r_{4}+$ $\ldots+r_{n}$, vemos que $r$ não pode variar em qualquer domínio de integração, mas apenas em uma certa região limitada.

Vale notar que neste problema prático, que nos motivou inicialmente, a estrutura da integral que apareceu não era exatamente aquela apresentada na Proposição C.16, pois apareciam duas funções de Bessel esféricas de ordem $l$ entre todas as outras de ordem dois. Para obter uma integral envolvendo apenas funções de ordem dois invocamos o resultado do Lema C.1. Esquematicamente, a aplicação deste resultado corresponderia a transformar os lados $X$ e $R$ (indicados na Figura C.5) em um novo lado $r$ e daí então recobraria-se um polígono exatamente como indicado na Figura C.3.

Neste problema o vôo aleatório corresponderia a um processo de espalhamentos múltiplos que fótons experimentaram durante a época da recombinação do universo. O afastamento da distribuição de equilíbrio térmico para a temperatura e polarização dos fótons da radiação cósmica de fundo é introduzida através destes espalhamentos devido a inomogeneidades dos potenciais gravitacionais, sobredensidades e velocidades peculiares dos elétrons do meio. Após percorrer as distâncias indicadas por $r_{n}, r_{n-1}, \ldots, r_{2}, r_{1}$ - e estando a uma distância $r$ do ponto onde iniciou o percurso - o fóton é dito desacoplado da matéria e propaga-se livremente até ser observado. Esta é a razão pela qual o triângulo formado pelos lados $R, X$ e $r$ na Figura C.5 é especialmente diferente dos outros do polígono superior. Como a distância entre o desacoplamento do fóton e sua observação é finita, um limite superior para o lado $r$ implica direta- 
150 Alguns resultados sobre funções de Bessel

mente um limite superior para o lado $X$ no triângulo $R, X, r$. Isso mostra que o polígono superior e esse triângulo destacado são umbilicamente relacionados, e essa relação implica que fontes capazes de causar efeitos sobre a temperatura e a polarização de fótons da radiação cósmica de fundo devem estar necessariamente dentro do cone de luz passado do observador desses fótons [4].

\section{C.4.2 Duas expansões da mesma função}

Como estamos particularmente interessados em integrais da forma (C.36) podemos realizar o mesmo procedimento ilustrado em (C.37) para obter integrais da forma ${ }^{4}$ :

$$
\begin{aligned}
F_{m, n}\left(X, R ; r_{1}, \ldots, r_{n}\right)= & \frac{(-1)^{m}}{2} \int d r\left(\frac{R X}{r}\right)^{m-1} \frac{1}{R^{m}} P_{l}^{-m}(\cos \alpha) \operatorname{sen}^{m} \alpha \\
& \times \int_{0}^{\infty} d k k^{2} j_{m}(k r) \frac{j_{m}\left(k r_{1}\right)}{\left(k r_{1}\right)^{m}} \frac{j_{m}\left(k r_{2}\right)}{\left(k r_{2}\right)^{m}} \ldots \frac{j_{m}\left(k r_{n-1}\right)}{\left(k r_{n-1}\right)^{m}} j_{m}\left(k r_{n}\right) .
\end{aligned}
$$

Aprendemos, entretanto, de (C.33), que a última integral que aparece nesta expressão pode ser escrita em termos da série de Fourier-Bessel:

$$
\begin{aligned}
F_{m, n}\left(X, R ; r_{1}, \ldots, r_{n}\right)= & \frac{(-1)^{m}}{2} \int d r\left(\frac{R X}{r}\right)^{m-1} \frac{1}{R^{m}} P_{l}^{-m}(\cos \alpha) \operatorname{sen}^{m} \alpha \sum_{q=1}^{\infty} \frac{\pi}{a^{3} j_{m+1}^{2}\left(x_{m q} a\right)} \\
& \times j_{m}\left(x_{m q} r\right) \frac{j_{m}\left(x_{m q} r_{1}\right)}{\left(x_{m q} r_{1}\right)^{m}} \frac{j_{m}\left(x_{m q} r_{2}\right)}{\left(x_{m q} r_{2}\right)^{m}} \ldots \frac{j_{m}\left(x_{m q} r_{n-1}\right)}{\left(x_{m q} r_{n-1}\right)^{m}} j_{m}\left(x_{m q} r_{n}\right) \text { (.C.38) }
\end{aligned}
$$

O caso particular em que $n=1$ desta expressão resulta:

$$
F_{m, 1}\left(X, R ; r_{1}\right)=\frac{(-1)^{m}}{2} \int d r\left(\frac{R X}{r}\right)^{m-1} \frac{1}{R^{m}} P_{l}^{-m}(\cos \alpha) \operatorname{sen}^{m} \alpha \frac{\pi}{a^{3}} \sum_{q=1}^{\infty} \frac{j_{m}\left(x_{m q} r\right) j_{m}\left(x_{m q} r_{1}\right)}{j_{m+1}^{2}\left(x_{m q} a\right)} .
$$

Desejamos agora escrever a série de Fourier-Bessel na variável $X$ da mesma função, mas sem ter anteriormente eliminado os lados $R$ e $X$ :

$F_{m, n}\left(X, R ; r_{1}, \ldots, r_{n}\right)=\int_{0}^{\infty} d k k^{2} \frac{j_{l}(k R)}{(k R)^{m}} j_{l}(k X) \frac{j_{m}\left(k r_{1}\right)}{\left(k r_{1}\right)^{m}} \frac{j_{m}\left(k r_{2}\right)}{\left(k r_{2}\right)^{m}} \ldots \frac{j_{m}\left(k r_{n-1}\right)}{\left(k r_{n-1}\right)^{m}} j_{m}\left(k r_{n}\right)$.

Como discutimos anteriormente, também neste caso a variável $X$ será limitada superiormente pela soma $R+r_{1}+r_{2}+\ldots+r_{n}$ (que chamaremos de $\tilde{a}$ ). Portanto,

\footnotetext{
${ }^{4}$ Tendo substituído o índice 2 que aparece na integral de interesse por um índice $m$ genérico, $m \leq l$.
} 


$$
\begin{aligned}
F_{m, n}\left(X, R ; r_{1}, \ldots, r_{n}\right)= & \sum_{q=1}^{\infty} \frac{\pi}{\tilde{a}^{3} j_{l+1}^{2}\left(\tilde{x}_{l q} \tilde{a}\right)} \frac{j_{l}\left(\tilde{x}_{l q} R\right)}{\left(\tilde{x}_{l q} R\right)^{m}} j_{l}\left(\tilde{x}_{l q} X\right) \\
& \times \frac{j_{m}\left(\tilde{x}_{l q} r_{1}\right)}{\left(\tilde{x}_{l q} r_{1}\right)^{m}} \frac{j_{m}\left(\tilde{x}_{l q} r_{2}\right)}{\left(\tilde{x}_{l q} r_{2}\right)^{m}} \ldots \frac{j_{m}\left(\tilde{x}_{l q} r_{n-1}\right)}{\left(\tilde{x}_{l q} r_{n-1}\right)^{m}} j_{m}\left(\tilde{x}_{l q} r_{n}\right)
\end{aligned}
$$

onde

$$
\tilde{x}_{l q}:=\frac{\alpha_{q}^{l}}{\tilde{a}}
$$

e $\alpha_{q}^{l}$ é, como antes, o q-ésimo zero da função de Bessel esférica de ordem $l$.

Podemos, invocando o Lema C.1, reescrever (C.40):

$$
\begin{aligned}
F_{m, n}\left(X, R ; r_{1}, \ldots, r_{n}\right)= & \sum_{q=1}^{\infty} \frac{\pi}{\tilde{a}^{3} j_{l+1}^{2}\left(\tilde{x}_{l q} \tilde{a}\right)} \frac{1}{\left(\tilde{x}_{l q} R\right)^{m}} \frac{j_{m}\left(\tilde{x}_{l q} r_{1}\right)}{\left(\tilde{x}_{l q} r_{1}\right)^{m}} \frac{j_{m}\left(\tilde{x}_{l q} r_{2}\right)}{\left(\tilde{x}_{l q} r_{2}\right)^{m}} \ldots \frac{j_{m}\left(\tilde{x}_{l q} r_{n-1}\right)}{\left(\tilde{x}_{l q} r_{n-1}\right)^{m}} j_{m}\left(\tilde{x}_{l q} r_{n}\right) \\
& \times \frac{(-1)^{m}}{2} \int d r\left(\tilde{x}_{l q}\right)^{m}\left(\frac{R X}{r}\right)^{m-1} P_{l}^{-m}(\cos \alpha) \operatorname{sen}^{m} \alpha j_{m}\left(\tilde{x}_{l q} r\right) \\
= & \frac{(-1)^{m}}{2} \frac{\pi}{\tilde{a}^{3}} \int d r \frac{1}{R^{m}}\left(\frac{R X}{r}\right)^{m-1} P_{l}^{-m}(\cos \alpha) \operatorname{sen}^{m} \alpha \\
& \times \sum_{q=1}^{\infty} \frac{1}{j_{l+1}^{2}\left(\tilde{x}_{l q} \tilde{a}\right)} j_{m}\left(\tilde{x}_{l q} r\right) \frac{j_{m}\left(\tilde{x}_{l q} r_{1}\right)}{\left(\tilde{x}_{l q} r_{1}\right)^{m}} \frac{j_{m}\left(\tilde{x}_{l q} r_{2}\right)}{\left(\tilde{x}_{l q} r_{2}\right)^{m}} \ldots \frac{j_{m}\left(\tilde{x}_{l q} r_{n-1}\right)}{\left(\tilde{x}_{l q} r_{n-1}\right)^{m}} j_{m}\left(\tilde{x}_{l q} r_{n}\right)
\end{aligned}
$$

Aqui, $r^{2}=R^{2}+X^{2}-2 R X \cos \alpha$.

Para verificar a implicação desta forma de escrever $F_{m, n}\left(X, R ; r_{1}, \ldots, r_{n}\right)$, consideremos os casos especiais:

Para $n=1$, $F_{m, 1}\left(X, R ; r_{1}\right)=\frac{(-1)^{m}}{2} \int d r \frac{1}{R^{m}}\left(\frac{R X}{r}\right)^{m-1} P_{l}^{-m}(\cos \alpha) \operatorname{sen}^{m} \alpha \frac{\pi}{\tilde{a}^{3}} \sum_{q=1}^{\infty} \frac{j_{m}\left(\tilde{x}_{l q} r_{1}\right) j_{m}\left(\tilde{x}_{l q} r\right)}{j_{l+1}^{2}\left(\tilde{x}_{l q} \tilde{a}\right)}$

Tomemos agora a diferença entre (C.39) e (C.41):

$$
\begin{aligned}
0= & F_{m, 1}\left(X, R ; r_{1}\right)-F_{m, 1}\left(X, R ; r_{1}\right)=\frac{(-1)^{m}}{2} \int d r \frac{1}{R^{m}}\left(\frac{R X}{r}\right)^{m-1} P_{l}^{-m}(\cos \alpha) \operatorname{sen}^{m} \alpha \\
& \times\left[\frac{\pi}{\tilde{a}^{3}} \sum_{q=1}^{\infty} \frac{j_{m}\left(\tilde{x}_{l q} r_{1}\right) j_{m}\left(\tilde{x}_{l q} r\right)}{j_{l+1}^{2}\left(\tilde{x}_{l q} \tilde{a}\right)}-\frac{\pi}{a^{3}} \sum_{q=1}^{\infty} \frac{j_{m}\left(x_{m q} r\right) j_{m}\left(x_{m q} r_{1}\right)}{j_{m+1}^{2}\left(x_{m q} a\right)}\right] .
\end{aligned}
$$

Como $\frac{1}{R^{m}}\left(\frac{R X}{r}\right)^{m-1} P_{l}^{-m}(\cos \alpha) \operatorname{sen}^{m} \alpha$ não se anula identicamente para todo $m, l, l \geq m$, no intervalo de integração da variável $r$, concluímos que deve valer a identidade: 


$$
\frac{\pi}{\tilde{a}^{3}} \sum_{q=1}^{\infty} \frac{j_{m}\left(\tilde{x}_{l q} r_{1}\right) j_{m}\left(\tilde{x}_{l q} r\right)}{j_{l+1}^{2}\left(\tilde{x}_{l q} \tilde{a}\right)}-\frac{\pi}{a^{3}} \sum_{q=1}^{\infty} \frac{j_{m}\left(x_{m q} r\right) j_{m}\left(x_{m q} r_{1}\right)}{j_{m+1}^{2}\left(x_{m q} a\right)} .
$$

Pela relação de completeza para as funções de Bessel (C.34) concluímos, então, que:

$$
\frac{\pi}{\tilde{a}^{3}} \sum_{q=1}^{\infty} \frac{j_{m}\left(\tilde{x}_{l q} r_{1}\right) j_{m}\left(\tilde{x}_{l q} r\right)}{j_{l+1}^{2}\left(\tilde{x}_{l q} \tilde{a}\right)}=\frac{\pi}{2 r^{2}} \delta\left(r-r_{1}\right) .
$$

Embora a forma de (C.44) seja muito próxima a (C.34), deve-se notar que aqui comparecem os zeros da função de Bessel de ordem $l$ nos argumentos das funções de Bessel de ordem $m$, enquanto lá compareciam sempre os zeros de mesma ordem que as funções de Bessel no denominador da expressão.

Podemos verificar que a condição (C.44) é suficiente para que $F_{m, 2}\left(X, R ; r_{1}, r_{2}\right)$ dada tanto por (C.39) quanto por (C.41) sejam identicas:

$$
\begin{aligned}
F_{m, 2}\left(X, R ; r_{1}, r_{2}\right)= & \frac{(-1)^{m}}{2} \frac{\pi}{\tilde{a}^{3}} \int d r \frac{1}{R^{m}}\left(\frac{R X}{r}\right)^{m-1} P_{l}^{-m}(\cos \alpha) \operatorname{sen}^{m} \alpha \\
& \times \sum_{q=1}^{\infty} \frac{1}{j_{l+1}^{2}\left(\tilde{x}_{l q} \tilde{a}\right)} j_{m}\left(\tilde{x}_{l q} r\right) \frac{j_{m}\left(\tilde{x}_{l q} r_{1}\right)}{\left(\tilde{x}_{l q} r_{1}\right)^{m}} j_{m}\left(\tilde{x}_{l q} r_{2}\right) \\
= & \frac{(-1)^{m}}{2} \frac{\pi}{\tilde{a}^{3}} \int d r \frac{1}{R^{m}}\left(\frac{R X}{r}\right)^{m-1} P_{l}^{-m}(\cos \alpha) \operatorname{sen}^{m} \alpha \sum_{q=1}^{\infty} \frac{1}{j_{l+1}^{2}\left(\tilde{x}_{l q} \tilde{a}\right)} \frac{j_{m}\left(\tilde{x}_{l q} r\right)}{\left(\tilde{x}_{l q} r_{1}\right)^{m}} \\
& \times \frac{(-1)^{m}}{2} \int d \rho\left(\tilde{x}_{l q}\right)^{m}\left(\frac{r_{1} r_{2}}{\rho}\right)^{m-1} P_{m}^{-m}\left(\cos \alpha_{1}\right) \operatorname{sen}^{m} \alpha_{1} j_{m}\left(\tilde{x}_{l q} \rho\right) \\
= & \frac{\pi}{4 \tilde{a}^{3}} \frac{1}{R^{m} r_{1}^{m}} \int d r \int d \rho \frac{\left(R X r_{1} r_{2}\right)^{m-1}}{(r \rho)^{m-1}} P_{l}^{-m}(\cos \alpha) \operatorname{sen}^{m} \alpha \\
& \times P_{m}^{-m}\left(\cos \alpha_{1}\right) \operatorname{sen}^{m} \alpha_{1} \sum_{q=1}^{\infty} \frac{j_{m}\left(\tilde{x}_{l q} r\right) j_{m}\left(\tilde{x}_{l q} \rho\right)}{j_{l+1}^{2}\left(\tilde{x}_{l q} \tilde{a}\right)}
\end{aligned}
$$

e por (C.44), temos:

$$
F_{m, 2}(X)=\frac{\pi}{8} \frac{1}{R^{m} r_{1}^{m}} \int d r \frac{\left(R X r_{1} r_{2}\right)^{m-1}}{(r)^{2 m}} P_{l}^{-m}(\cos \alpha) \operatorname{sen}^{m} \alpha P_{m}^{-m}\left(\cos \alpha_{1}\right) \operatorname{sen}^{m} \alpha_{1},
$$

com $r^{2}=r_{1}^{2}+r_{2}^{2}-2 r_{1} r_{2} \cos \alpha_{1}$ e $r^{2}=R^{2}+X^{2}-2 R X \cos \alpha$. Esta identidade é exatamente a verificada no Lema C.3 e portanto as duas maneiras de escrever $F_{m, 2}\left(X, R ; r_{1}, r_{2}\right)$ coincidem. Com efeito, pelo mesmo procedimento aqui realizado mostra-se que a condição suficiente para que haja igualdade ordem a ordem das duas maneiras de escrever $F_{m, n}\left(X, R ; r_{1}, \ldots, r_{n}\right)$ é que (C.44) seja satisfeita. 
Pelo desenvolvimento que acabamos de apresentar, demonstramos uma identidade que havia sido conjecturada em [4] e cuja demonstração não era conhecida. Resumindo, vale curiosa relação tipo-completeza satisfeita por funções não-ortogonais:

Proposição C.8 Sejam $r, r_{1}$ e ã números reais positivos e $\tilde{x}_{l q}=\alpha_{q}^{l} / \tilde{a}$, onde $\alpha_{q}^{l}$ são as raízes da função de Bessel esférica de ordem l. Então vale a relação:

$$
\sum_{q=1}^{\infty} \frac{1}{\tilde{a}^{3}} \frac{j_{m}\left(\tilde{x}_{l q} r\right) j_{m}\left(\tilde{x}_{l q} r_{1}\right)}{j_{l+1}^{2}\left(\tilde{x}_{l q} \tilde{a}\right)}=\frac{1}{2 r_{1}^{2}} \delta\left(r_{1}-r\right)
$$

Um teorema demonstrado em [80] afirma que $S e \nu>-1 / 2$, o conjunto $\left\{x^{1 / 2} J_{\nu}\left(\lambda_{n} x\right)\right\}, n \in \mathbb{N}$, forma uma sequência completa (e portanto total) em $L^{p}(0,1), 1 \leq p<\infty$, se para todo $n$ suficientemente grande for verificada a desigualdade:

$$
0<\lambda_{n} \leq \pi\left(n+\frac{1}{4}+\frac{\nu}{2}-\frac{1}{2 p}\right) .
$$

Completaza, no sentido do teorema, significa que se $\int_{0}^{1} x^{1 / 2} J_{\nu}\left(\lambda_{n} x\right) g(x) d x=0$ para todo $\nu>-1 / 2$, com $g(x) \in L^{p}[0,1]$, então $g(x)$ anula-se em quase toda parte em $(0,1)[81]$.

Para grandes valores do argumento, as funções de Bessel $J_{\nu}(z)$ comportamse $\operatorname{como} \cos (z-\nu \pi / 2-\pi / 4)$ [53]. Assim, podemos concluir do teorema que o conjunto $\left\{x^{1 / 2} J_{\nu}\left(\lambda_{n} x\right)\right\}$ é uma sequência completa no espaço de Hilbert $L^{2}[0,1]$ quando os $\lambda_{n}$ são tomados como os zeros reais positivos de $J_{\mu}(z)$ - mas apenas se $\mu \leq \nu$. Em nosso caso, entretanto, $l \geq m$ (que corresponde a $\mu \geq \nu$ ) e portanto mostramos uma relação tipo-completeza para um conjunto de funções que não sabemos se é completo ou não (e muito provavelmente não seja) pois insere-se fora dos limites de validade do teorema transcrito e não conhecemos extensões deste teorema. Não sabemos tampouco como interpretar esta relação tipo-completeza no contexto geral do estudo de completeza (ou não) de conjuntos de funções de Bessel esféricas em espaços $L^{p}[0,1]$. 


\section{Apêndice D \\ Funções de spin e geometria na esfera}

Este apêndice pretende reunir elementos necessários à discussão das expansões de funções com spin como também para realizar-se as expansões de Taylor que introduzimos quando tratamos o efeito de lentes gravitacionais sobre a temperatura e polarização da CMB. Iniciamos apresentando o teorema de Peter-Weyl e a justificativa de que expansões de funções de spin fazem sentido. Introduziremos então os harmônicos esféricos de spin através dos elementos da representação irredutível unitária de $\mathrm{SO}(3)$ e os operadores ð e $\bar{\partial}$. Mostraremos que estes operadores são uma forma de representar as derivadas covariantes em direções ortogonais sobre a esfera $S^{2}$. Incluiremos, por fim, um conjunto de fórmulas envolvendo coeficientes de Clebsch-Gordan e características de $D_{m m^{\prime}}^{j}$ que foram usadas ao longo de todo o texto.

\section{D.1 Representações irredutíveis unitárias de $\mathrm{SO}(3)$ e funções de spin}

Prentendemos nesta seção justificar os tipos de expansões em termos de harmônicos esféricos de spin realizadas e sua intercambialidade com os elementos $D_{m m^{\prime}}^{l}$ que surgem no estudo das representações irredutíveis unitárias do grupo $\mathrm{SO}(3)$. Partiremos do teorema de Peter-Weyl.

\section{D.1.1 O teorema de Peter-Weyl}

Consideremos o teorema de Peter-Weyl [42]:

Teorema D.1 Seja $\left\{\Pi^{\alpha}, \alpha \in \Lambda\right\}$ a coleção de todas as representações unitárias irredutiveis de dimensão finita não-equivalentes entre si de um grupo compacto G. Sejam $\Pi^{\alpha}(g)_{i, j}, i, j=1, \ldots, d_{\alpha}$ seus elementos de matriz. Seja $d \mu$ a medida de Haar de G. Então

$$
\int_{G} \Pi^{\alpha}(g)_{i j}^{*} \Pi^{\beta}(g)_{k l} d \mu(g)=\frac{1}{d_{\alpha}} \delta_{\alpha \beta} \delta_{i k} \delta_{j l} .
$$


Por fim, as funções $\Pi^{\alpha}(g)_{i, j} i, j=1, \ldots, d_{\alpha}$ formam uma base ortogonal completa no espaço de Hilbert $\mathcal{L}^{2}(G, d \mu)$. Com isso, toda função $f \in \mathcal{L}^{2}(G, d \mu)$ pode ser escrita na forma

$$
f(g)=\sum_{\alpha \in \Lambda} \sum_{i, j=1}^{d_{\alpha}} a_{i j}^{\alpha} \Pi^{\alpha}(g)_{i j}
$$

onde

$$
a_{i j}^{\alpha}=d_{\alpha} \int_{G} \Pi^{\alpha}(g)_{i j}^{*} f(g) d \mu(g) .
$$

Ainda, para $f \in \mathcal{L}^{2}(G, d \mu)$ vale a identidade de Parseval:

$$
\int_{G}|f(g)|^{2} d \mu(g)=\sum_{\alpha \in \Lambda} \frac{1}{d_{\alpha}} \sum_{i, j=1}^{d_{\alpha}}\left|a_{i j}^{\alpha}\right|^{2} .
$$

Um operador com a propriedade de comutar com todos os geradores de um grupo de Lie é dito ser um operador de Casimir. Demonstra-se que $L^{2}$ é o único operador de Casimir não trivial de $S O(3)$. Como $L^{2}$ comuta com cada $L_{a}$, tendo $\Pi(R(\theta, \vec{\eta}))=\exp (-i \theta \vec{\eta} \cdot \vec{L})$, segue que $L^{2} \Pi(g)=\Pi(g) L^{2}, \forall g \in S O(3)$. Assim, pelo Lema de Schur, se $\Pi$ é uma representação irredutível, $L^{2}$ deve ser um múltiplo da identidade. Isso abre caminho para classificar as representações irredutíveis de $S O(3)$ : estudando os possíveis valores de $L^{2}$. Em cada subespaço formado por autovetores com um dado autovalor fixo, teremos uma representação irredutível.

No caso de $S O(3)$, os $\Pi^{\alpha}(g)$ devem ser identificados com as matrizes de rotação $D^{l}(R)$, sendo $d_{\alpha}=2 l+1$. Os $\Pi_{i, j}^{\alpha}(g), i, j=1, \ldots, d_{\alpha}$ serão os $D_{m m^{\prime}}^{l}(R)$, $m, m^{\prime}=-l, \ldots, l$.

As matrizes de rotação podem ser parametrizadas por três ângulos de Euler. Entretanto, se tomamos uma direção $\mathbf{n}$ caracterizada pelos ângulos $(\theta, \phi)$ com $\mathbf{n} \cdot \mathbf{n}_{z}=\cos \theta$, essa direção pode ser obtida de $\mathbf{n}_{z}$ por uma rotação $\mathbf{n}_{z} \rightarrow \mathbf{n}$

$$
\mathbf{n} \rightarrow \mathrm{e}^{-i \phi J_{z}} \mathrm{e}^{-i \theta J_{y}} \mathbf{n}_{\mathbf{z}}=D(\phi, \theta, 0) \mathbf{n}_{z} .
$$

Basta, portanto, fixar dois ângulos para definir completamente uma segunda direção a partir da primeira. Fixar o ângulo de Euler $\alpha=\phi$ e $\beta=\theta$ é necessário. $\gamma$, entretanto, não é fixado pela imposição $\mathbf{n}_{z} \rightarrow \mathbf{n}$.

Há, entretanto, uma classe de funções que se caracteriza por seu comportamento sob rotação em torno de $\mathbf{n}$ por um ângulo arbitrário. Essas funções 
156 Funções de spin e geometria na esfera

satisfazem $f(x) \rightarrow f(x) \mathrm{e}^{-i m_{0} \gamma}$, onde $x$ é a coleção de parâmetros de que $f$ explicitamente depende, $m_{0}$ é chamado de spin e $\gamma$ é o ângulo de rotação. Essas funções são chamadas de funções de spin $m_{0}$ [82], [83]. Consideremos uma dessas funções com spin.

Seja $f(\alpha, \beta) \mathrm{e}^{-i m_{0} \gamma}$ com $\alpha, \beta$ e $\gamma$ ângulos de Euler. Pelo teorema de PeterWeyl,

$$
f(\alpha, \beta) \mathrm{e}^{-i m_{0} \gamma}=\sum_{j=0}^{\infty} \sum_{m, m^{\prime}=-j}^{j} c_{m m^{\prime}}^{j} D_{m m^{\prime}}^{j}(\alpha, \beta, \gamma)
$$

com

$$
\begin{aligned}
c_{m m^{\prime}}^{j} & =(2 j+1) \int D_{m m^{\prime}}^{j *}(\alpha, \beta, \gamma) f(\alpha, \beta) \mathrm{e}^{-i m_{0} \gamma} d \mu \\
& =(2 j+1) \int_{0}^{4 \pi} \frac{d \alpha}{4 \pi} \int_{0}^{4 \pi} \frac{d \gamma}{4 \pi} \int_{0}^{\pi} \frac{d \beta}{2} \operatorname{sen} \beta \mathrm{e}^{i m \alpha} d_{m m^{\prime}}^{j}(\beta) \mathrm{e}^{i\left(m^{\prime}-m_{0}\right) \gamma} f(\alpha, \beta) \\
& =(2 j+1) \int_{0}^{4 \pi} \frac{d \gamma}{4 \pi} \mathrm{e}^{i\left(m^{\prime}-m_{0}\right) \gamma} \int_{0}^{4 \pi} \frac{d \alpha}{4 \pi} \int_{0}^{\pi} \frac{d \beta}{2} \operatorname{sen} \beta D_{m m_{0}}^{j *}(\alpha, \beta, 0) f(\alpha, \beta) \\
& =(2 j+1) \int_{0}^{4 \pi} \frac{d \alpha}{4 \pi} \int_{0}^{\pi} \frac{d \beta}{2} \operatorname{sen} \beta D_{m m^{\prime}}^{j *}(\alpha, \beta, 0) f(\alpha, \beta) \\
& =(2 j+1) c_{m m_{0}}^{j} \equiv a_{m}^{j},
\end{aligned}
$$

ou seja,

$$
\begin{aligned}
f(\alpha, \beta) & =\sum_{j, m}(2 j+1) c_{m m_{0}}^{j} \mathrm{e}^{i m_{0} \gamma} D_{m m_{0}}^{j}(\alpha, \beta, \gamma) \\
& =\sum_{j, m} a_{m}^{j} D_{m m_{0}}^{j}(\alpha, \beta, 0) .
\end{aligned}
$$

Reescrevendo,

$$
f(\alpha, \beta)=\sum_{j, m} a_{m}^{j} D_{m m_{0}}^{j}(\alpha, \beta, 0)
$$

com

$$
a_{m}^{j}=(2 j+1) \frac{1}{4 \pi} \int_{0}^{2 \pi} d \alpha \int_{0}^{\pi} d \beta \operatorname{sen} \beta D_{m m_{0}}^{j *}(\alpha, \beta, 0) f(\alpha, \beta) .
$$

As funções $D_{m m^{\prime}}^{j}(\alpha, \beta, 0)$ são ortogonais pois, quando um produto de duas tais funções são integradas nos ângulos $\alpha$ e $\beta$, as exponenciais (que dependem de $\alpha$ ) são ortogonais e as funções $d_{m m^{\prime}}^{j}(\beta)$, que são polinômios de Jacobi, são 
também polinômios ortogonais. Esta é a origem de poder-se fazer expansões em termos do que definiremos ser harmônicos esféricos de spin.

Efeito de uma rotação dos vetores de base. Consideremos uma rotação de um ângulo $\gamma$ do sistema de coordenadas determinado pelo par de vetores ortonormais $\mathbf{e}_{1}, \mathbf{e}_{2}$, ou seja, façamos $\mathbf{e}_{1} \rightarrow \cos \gamma \mathbf{e}_{1}-\operatorname{sen} \gamma \mathbf{e}_{2}$ e $\mathbf{e}_{2} \rightarrow \operatorname{sen} \gamma \mathbf{e}_{1}-\cos \gamma \mathbf{e}_{2}$. Analisemos então o comportamento das seguintes combinações de $\mathbf{e}_{1}, \mathbf{e}_{2}$ :

$$
\mathbf{e}_{+}^{\prime}=-\frac{1}{\sqrt{2}}\left(\mathbf{e}_{1}^{\prime}+i \mathbf{e}_{2}^{\prime}\right)=-\frac{1}{\sqrt{2}}\left(\cos \gamma \mathbf{e}_{1}-\operatorname{sen} \gamma \mathbf{e}_{2}+i \operatorname{sen} \gamma \mathbf{e}_{1}+i \cos \gamma \mathbf{e}_{2}\right)=e^{i \gamma} \mathbf{e}_{+} .
$$

Analogamente $\mathbf{e}_{-}^{\prime}=e^{-i \gamma} \mathbf{e}_{-}$.

Assim, se temos uma quantidade vetorial $\mathbf{V}$ decomposta na base $\left\{\mathbf{e}_{+}, \mathbf{e}_{-}\right\}$, ou seja, $\mathbf{V}=V^{+} \mathbf{e}_{+}+V^{-} \mathbf{e}_{-}$, as componentes $V^{+}$e $V^{-}$devem se comportar como $V^{+^{\prime}}=e^{-i \gamma} V^{+}$e $V^{-^{\prime}}=e^{i \gamma} V^{-}$sob uma rotação como a acima descrita.

Se escrevemos a parte sem traço da matriz densidade descrevendo a polarização de um feixe de fótons na base $\mathbf{e}_{+}, \mathbf{e}_{-}$, teremos:

$$
\mathcal{P}=\left(\begin{array}{cc}
\frac{Q+i U}{4 I} & 0 \\
0 & \frac{Q-i U}{4 I}
\end{array}\right),
$$

e, daí, temos que $\mathcal{P}^{++^{\prime}}=\left(\frac{Q+i U}{4 I}\right)=e^{-2 i \gamma} \mathcal{P}^{++}$e $\mathcal{P}^{--^{\prime}}=\left(\frac{Q-i U}{4 I}\right)=e^{+2 i \gamma} \mathcal{P}^{--}$. Logo, de acordo com nossas definições, $\frac{Q-i U}{4 I}$ descreve um objeto de spin 2 e $\frac{Q+i U}{4 I}$, um objeto de spin $-2^{1}$.

\section{D.1.2 Relação entre matrizes de rotação e harmônicos esféricos de spin}

Definindo os harmônicos esféricos de spin da maneira mais direta possível à partir dos $D_{m^{\prime} m}^{j}$, seguindo o fato que

$$
D_{m 0}^{j *}(\alpha, \beta, \gamma)=\sqrt{\frac{4 \pi}{2 j+1}} \mathrm{Y}_{j m}(\beta, \alpha),
$$

definimos

$$
D_{m^{\prime}-m}^{j *}(\alpha, \beta, \gamma)=: \sqrt{\frac{4 \pi}{2 j+1}} m \mathrm{Y}_{j m^{\prime}}(\beta, \alpha)
$$

impondo-se $\gamma=0$.

\footnotetext{
${ }^{1}$ Lembrar que entendemos que uma função tenha spin $m_{0}$ se, quando executada uma rotação de um ângulo $\gamma$ a função satisfaz $f(x) \rightarrow f(x) \mathrm{e}^{-i m_{0} \gamma}$, onde $x$ é a coleção de parâmetros de que $f$ explicitamente depende.
} 
158 Funções de spin e geometria na esfera

O objeto ${ }_{m} \mathrm{Y}_{j m^{\prime}}(\beta, \alpha)$ é chamado de harmônico esférico de spin $m$.

Convém notar que a conexão dos harmônicos esféricos de spin com matrizes de rotação aparece de maneira não-homogênea na literatura e não é difícil encontrar diferentes convenções para definí-los (ver [82], [84], [49] ou [85] para alguns exemplos).

\section{D.1.3 Operadores de levantamento e abaixamento de spin}

No estudo da teoria do momento angular estuda-se amplamente a ação de operadores $\mathcal{J}_{1}, \mathcal{J}_{2}$ e $\mathcal{J}_{3}$ sobre os elementos da representação do grupo de rotações. Em termos das combinações $\mathcal{J}_{+}=\mathcal{J}_{1}+i \mathcal{J}_{2}$ e $\mathcal{J}_{-}=\mathcal{J}_{1}-i \mathcal{J}_{2}$, escrevemos a forma explícita:

$$
\begin{aligned}
& \mathcal{J}_{+}=\mathrm{e}^{i \alpha}\left(i \operatorname{cotg} \beta \frac{\partial}{\partial \alpha}+\frac{\partial}{\partial \beta}-\frac{i}{\operatorname{sen} \beta} \frac{\partial}{\partial \gamma}\right) \\
& \mathcal{J}_{-}=\mathrm{e}^{-i \alpha}\left(i \operatorname{cotg} \beta \frac{\partial}{\partial \alpha}-\frac{\partial}{\partial \beta}-\frac{i}{\operatorname{sen} \beta} \frac{\partial}{\partial \gamma}\right) \\
& \mathcal{J}_{3}=-i \frac{\partial}{\partial \alpha} .
\end{aligned}
$$

Donde seguem as ações sobre $D_{m^{\prime} m}^{j}$ :

$$
\begin{aligned}
\mathcal{J}_{ \pm} D_{m^{\prime} m}^{j *}(\alpha, \beta, \gamma) & =\left[\left(j \mp m^{\prime}\right)\left(j \pm m^{\prime}+1\right)\right]^{1 / 2} D_{\left(m^{\prime} \pm 1\right) m}^{j *}(\alpha, \beta, \gamma) \\
\mathcal{J}_{3} D_{m^{\prime} m}^{j *}(\alpha, \beta, \gamma) & =m^{\prime} D_{m^{\prime} m}^{j *}(\alpha, \beta, \gamma) .
\end{aligned}
$$

Há, entretanto, um segundo conjunto completo de operadores compatíveis que induzirá mudanças no segundo índice das $D_{m^{\prime} m}^{j}$. Seguindo [86] definimos:

$$
\begin{gathered}
\mathcal{K}_{+}=\left(\mathcal{K}_{1}+i \mathcal{K}_{2}\right)=\mathrm{e}^{-i \gamma}\left(-i \operatorname{cotg} \beta \frac{\partial}{\partial \gamma}+\frac{\partial}{\partial \beta}+\frac{i}{\operatorname{sen} \beta} \frac{\partial}{\partial \alpha}\right), \\
\mathcal{K}_{-}=\left(\mathcal{K}_{1}-i \mathcal{K}_{2}\right)=\mathrm{e}^{i \gamma}\left(-i \operatorname{cotg} \beta \frac{\partial}{\partial \gamma}-\frac{\partial}{\partial \beta}+\frac{i}{\operatorname{sen} \beta} \frac{\partial}{\partial \alpha}\right)
\end{gathered}
$$

e

$$
\mathcal{K}_{3}=-i \frac{\partial}{\partial \gamma}
$$

Pode-se mostrar [86] que esses operadores têm as seguintes propriedades: 


$$
\begin{aligned}
\mathcal{K}_{-} D_{m^{\prime} m}^{j *}(\alpha, \beta, \gamma) & =[(j-m)(j+m+1)]^{1 / 2} D_{m^{\prime}(m+1)}^{j *}(\alpha, \beta, \gamma) \\
\mathcal{K}_{+} D_{m^{\prime} m}^{j *}(\alpha, \beta, \gamma) & =[(j+m)(j-m+1)]^{1 / 2} D_{m^{\prime}(m-1)}^{j *}(\alpha, \beta, \gamma) \\
\mathcal{K}_{3} D_{m^{\prime} m}^{j *}(\alpha, \beta, \gamma) & =m D_{m^{\prime} m}^{j *}(\alpha, \beta, \gamma) .
\end{aligned}
$$

Segue também que:

$$
\left\{\frac{1}{2}\left[\mathcal{K}_{+} \mathcal{K}_{-}+\mathcal{K}_{-} \mathcal{K}_{+}\right]+\mathcal{K}_{3}^{2}\right\} D_{m^{\prime} m}^{j *}(\alpha, \beta, \gamma)=j(j+1) D_{m^{\prime} m}^{j *}(\alpha, \beta, \gamma) .
$$

Fazendo analogia com o problema do rotor em mecânica quântica, $\mathcal{J}_{ \pm}$aumentam ou diminuem a projeção do momento angular em relação ao eixo $z$ de um sistema de referência fixo enquanto $\mathcal{K}_{ \pm}$sobem ou descem a projeção do momento angular com relação ao eixo $z^{\prime}$ coincidindo com o eixo de simetria do rotor (que está em rotação com relação ao sistema sem ').

Define-se de maneira análoga a [82], fazendo referência a (D.19), os objetos

$$
\begin{gathered}
{\left[\mathcal{K}_{+} D_{m-s}^{j *}(\alpha, \beta, \gamma)\right]_{\gamma=0}=ð D_{m-s}^{j *}(\alpha, \beta, 0),} \\
{\left[-\mathcal{K}_{-} D_{m-s}^{j *}(\alpha, \beta, \gamma)\right]_{\gamma=0}=\bar{\varnothing} D_{m-s}^{j *}(\alpha, \beta, 0) .}
\end{gathered}
$$

O símbolo ð é chamado eth. O sinal de menos em (D.23) é para que se tenha concordância com o efeito desse operador sobre os harmônicos esféricos de spin tal como apresentado em [82] ou [83], ou seja, para que

$$
\begin{aligned}
ð_{m} \mathrm{Y}_{j m^{\prime}} & =[(j-m)(j+m+1)]^{1 / 2}{ }_{(m+1)} \mathrm{Y}_{j m^{\prime}} \\
\bar{\partial}_{m} \mathrm{Y}_{j m^{\prime}} & =-[(j+m)(j-m+1)]^{1 / 2}{ }_{(m-1)} \mathrm{Y}_{j m^{\prime}} .
\end{aligned}
$$

Claramente,

$$
ð D_{m-s}^{j *}(\alpha, \beta, 0)=[(j+m)(j-m+1)]^{1 / 2} D_{m^{\prime}-s-1}^{j *}(\alpha, \beta, 0)
$$

e

$$
\overline{\bar{\partial}} D_{m-s}^{j *}(\alpha, \beta, 0)=-[(j-m)(j+m+1)]^{1 / 2} D_{m^{\prime}-s+1}^{j *}(\alpha, \beta, 0) .
$$

Deve-se notar que

$$
\left(\mathcal{K}_{+}\right)^{*}=-\left(\mathcal{K}_{-}\right) \quad\left(\mathcal{K}_{-}\right)^{*}=-\left(\mathcal{K}_{+}\right)
$$


160 Funções de spin e geometria na esfera

e, portanto, pode-se entender também o sinal menos que aparece em (D.23) como uma maneira de fazer com que, de fato, ð e $\bar{\partial}$ sejam complexos conjugados um do outro já que $\mathcal{K}_{+}$e $\mathcal{K}_{-}$não o são.

\section{D.2 Símbolos de Christoffel na esfera}

Consideremos a esfera $S^{2}$ como nossa variedade de interesse. Podemos escrever a métrica de $S^{2}$ como

$$
g=d \theta \otimes d \theta+\operatorname{sen}^{2} \theta d \phi \otimes d \phi=\hat{\theta}^{1} \otimes \hat{\theta}^{1}+\hat{\theta}^{2} \otimes \hat{\theta}^{2}
$$

onde $\hat{\theta}^{1}=d \theta$ e $\hat{\theta}^{2}=\operatorname{sen} \theta d \phi$. Os zweibeine nesse caso são

$$
e_{1}^{\theta}=1 \quad e_{1}^{\phi}=0 \quad e_{2}^{\theta}=0 \quad e_{2}^{\phi}=\operatorname{sen} \theta .
$$

Passamos agora ao cálculo das 1-formas de conexão $\left\{\omega_{\beta}^{\alpha}\right\}$. Devemos notar, primeiro, que, considerando uma conexão de Levi-Civita, teremos, devido a (A.22), $\omega_{11}=\omega_{22}=0$, o que equivale a $\omega_{1}^{1}=\omega_{2}^{2}=0 . \omega_{2}^{1}$ e $\omega^{2}{ }_{1}$ podem ser obtidos com a ajuda da primeira equação de estrutura de Cartan.

$$
0=d(\operatorname{sen} \theta d \phi)+\omega^{2}{ }_{1} \wedge d \theta=\cos \theta d \theta \wedge d \phi+\omega^{2}{ }_{1} \wedge d \theta
$$

donde, $\omega^{2}{ }_{1}=\cos \theta d \phi$. Novamente usando (A.22), $\omega^{1}{ }_{2}=-\cos \theta d \phi$. Tendo calculado as 1-formas de conexão, podemos, usando (A.19), obter os símbolos de Christoffel. Os únicos símbolos não nulos são $\Gamma^{1}{ }_{22}=-\operatorname{cotg} \theta$ e $\Gamma^{1}{ }_{21}=\operatorname{cotg} \theta$.

Interessamo-nos, no entanto, em utilizar a base formada pelos vetores $\mathbf{e}_{+} \mathrm{e}$ e_ em nossos cálculos e, por isso, convém determinar os símbolos de Christoffel com relação a essa escolha de vetores base. Relembremos que:

$$
\mathbf{e}_{ \pm}=\mp \frac{1}{\sqrt{2}}\left(\mathbf{e}_{\theta} \pm i \mathbf{e}_{\phi}\right)
$$

onde simplificando a notação, deixamos de escrever o índice relacionado $k$.

Recobremos para essa empreitada a propriedade definidora dos símbolos de Christoffel, ou seja, $\nabla_{\mathbf{e}_{k}} \mathbf{e}_{j}=\Gamma_{k j}^{i} \mathbf{e}_{i}$, onde $i, j$ e $k$ podem ser + ou - .

Comecemos por $\nabla_{\mathbf{e}_{-}} \mathbf{e}_{+}$. 


$$
\begin{aligned}
& \nabla_{\mathbf{e}_{-}} \mathbf{e}_{+}=\nabla \frac{1}{\sqrt{2}}\left(\mathbf{e}_{\theta}-i \mathbf{e}_{\phi}\right) \\
&=-\frac{1}{2}\left(\mathbf{e}_{+}=-\frac{1}{\sqrt{2}} \nabla_{\frac{1}{\sqrt{2}}} \mathbf{e}_{\theta}+i \mathbf{e}_{\theta}-i \mathbf{e}_{\phi}\right) \\
&\left.\mathbf{e}_{\theta} \mathbf{e}_{\phi}-i \mathbf{e}_{\theta}+i \mathbf{e}_{\mathbf{e}_{\phi}} \mathbf{e}_{\theta}+\nabla_{\mathbf{e}_{\phi}} \mathbf{e}_{\phi}\right)= \\
&=-\frac{1}{2}\left[\Gamma_{11}^{1} \mathbf{e}_{1}+\Gamma_{11}^{2} \mathbf{e}_{2}+i\left(\Gamma_{12}^{1} \mathbf{e}_{1}+\Gamma_{12}^{2} \mathbf{e}_{2}\right)-i\left(\Gamma_{21}^{1} \mathbf{e}_{1}+\Gamma_{21}^{2} \mathbf{e}_{2}\right)+\Gamma_{22}^{1} \mathbf{e}_{1}+\Gamma_{22}^{2} \mathbf{e}_{2}\right]= \\
&=-\frac{1}{\sqrt{2}}(-\operatorname{cotg} \theta) \frac{\left(\mathbf{e}_{1}+i \mathbf{e}_{2}\right)}{\sqrt{2}}=\frac{-\operatorname{cotg} \theta}{\sqrt{2}}= \\
&=\Gamma_{-+}^{+} \mathbf{e}_{+} .
\end{aligned}
$$

De modo análogo obtém-se $\Gamma_{+-}^{-}=\frac{\operatorname{cotg} \theta}{\sqrt{2}}, \Gamma_{++}^{+}=\frac{-\operatorname{cotg} \theta}{\sqrt{2}}$ e $\Gamma_{--}^{-}=\frac{\operatorname{cotg} \theta}{\sqrt{2}}$, que completam o conjunto dos símbolos de Christoffel não nulos.

\section{D.3 Maneira de reescrever a derivada covariante}

Seja $T$ um tensor de posto 2 escrito na base $\mathbf{e}_{+}, \mathbf{e}_{-}$e que é diagonal nessa base $^{2}$. Desejamos obter a derivada covariante das componentes desse tensor com relação aos vetores da base, ou seja, desejamos obter $T_{;-}^{++}, T_{;+}^{++}, T_{;+}^{--}$e $T_{;--}^{--}$.

$$
T_{;+}^{--}=\mathbf{e}_{+}\left(T^{--}\right)+\Gamma_{+-}^{-} T^{--}+\Gamma_{+-}^{-} T^{--} .
$$

Onde usamos o fato que o tensor é diagonal e que os únicos símbolos de conexão não nulos são $\Gamma_{-+}^{+}=-\Gamma_{+-}^{-}=\Gamma_{++}^{+}=-\Gamma_{--}^{-}=-\frac{1}{\sqrt{2}} \operatorname{cotg} \theta$. Lembrando a maneira como operadores que atuam subindo e descendo índices de spin em harmônicos esféricos de spin são dados em termos de operadores diferenciais [87], teremos:

$$
\begin{aligned}
T_{;+}^{--} & =-\frac{1}{\sqrt{2}}\left(\partial_{\theta}+\frac{i}{\operatorname{sen} \theta} \partial_{\phi}\right) T^{--}+\frac{2}{\sqrt{2}} \operatorname{cotg} \theta T^{--}= \\
& =-\frac{1}{\sqrt{2}}\left(\partial_{\theta}-2 \operatorname{cotg} \theta+\frac{i}{\operatorname{sen} \theta} \partial_{\phi}\right) T^{--}= \\
& =\frac{1}{\sqrt{2}} ð T^{--} .
\end{aligned}
$$

Continuando,

${ }^{2} \mathrm{O}$ posto do tensor ser assumido 2 aqui é apenas uma conveniência, mas a generalização para o caso de um posto $s$ é imediato [49]. 


$$
\begin{aligned}
T_{;-}^{--} & =\mathbf{e}_{-}\left(T^{--}\right)+\Gamma_{--}^{-} T^{--}+\Gamma_{--}^{-} T^{--}= \\
& =\frac{1}{\sqrt{2}}\left(\partial_{\theta}-\frac{i}{\operatorname{sen} \theta} \partial_{\phi}\right) T^{--}+\frac{2}{\sqrt{2}} \operatorname{cotg} \theta T^{--}= \\
& =\frac{1}{\sqrt{2}}\left(\partial_{\theta}+2 \operatorname{cotg} \theta-\frac{i}{\operatorname{sen} \theta} \partial_{\phi}\right) T^{--}= \\
& =-\frac{1}{\sqrt{2}} \bar{\partial} T^{--}, \\
T_{;-}^{++} & =\mathbf{e}_{-}\left(T^{++}\right)+\Gamma_{-+}^{+} T^{++}+\Gamma_{-+}^{+} T^{++}= \\
& =\frac{1}{\sqrt{2}}\left(\partial_{\theta}-\frac{i}{\operatorname{sen} \theta} \partial_{\phi}\right) T^{++}-\frac{2}{\sqrt{2}} \operatorname{cotg} \theta T^{++}= \\
& =\frac{1}{\sqrt{2}}\left(\partial_{\theta}+(-2) \operatorname{cotg} \theta-\frac{i}{\operatorname{sen} \theta} \partial_{\phi}\right) T^{++}= \\
& =-\frac{1}{\sqrt{2}} \bar{\partial} T^{++}, \\
& =-\frac{1}{\sqrt{2}}\left(\partial_{\theta}-(-2) \operatorname{cotg} \theta+\frac{i}{\operatorname{sen} \theta} \partial_{\phi}\right) T^{++}= \\
T_{;+}^{++} & =\mathbf{e}_{+}\left(T^{++}\right)+\Gamma_{++}^{+} T^{++}+\Gamma_{++}^{+} T^{++}= \\
= & \frac{1}{\sqrt{2}}\left(\partial_{\theta}+\frac{i}{\operatorname{sen} \theta} \partial_{\phi}\right) T^{++}-\frac{2}{\sqrt{2}} \operatorname{cotg} \theta T^{++}= \\
& = \\
& \\
&
\end{aligned}
$$

Disso podemos depreender que:

$$
\nabla_{\mathbf{e}_{+}}=-\frac{1}{\sqrt{2}} \partial \quad \nabla_{\mathbf{e}_{-}}=\frac{1}{\sqrt{2}} \overline{\bar{\partial}} .
$$

Essa maneira de reescrever o operador de derivação é fundamental para que se possa escrever expansões das componentes de tensores, em particular, permite-nos fazer expansão de Taylor do tensor de polarização. Note-se que a diferença entre as identificações das derivadas com operadores atuando sobre as matrizes de rotação aqui apresentadas e as de [49], por exemplo, advém da 

diferente convenção adotada para a definição de $\mathbf{e}_{+}$e $\mathbf{e}_{-}$.

\section{D.4 Relações envolvendo $D_{m m^{\prime}}^{j}$ s e coeficientes de Clebsch-Gordan}

Apenas por conveniência reunimos algumas identidades sobre coeficientes de Clebsch-Gordan e identidades utilizadas de $D_{m m^{\prime}}^{j}$. Estas identidades podem ser encontradas na literatura, por exemplo, em [88], [89] ou [86].

$$
\begin{gathered}
\left\langle j_{2} m_{2} j_{1} m_{1} \mid J M\right\rangle=(-1)^{\left(j_{1}+j_{2}-J\right)}\left\langle j_{1} m_{1} j_{2} m_{2} \mid J M\right\rangle . \\
\left\langle j_{1}-m_{1} j_{2}-m_{2} \mid J-M\right\rangle=(-1)^{\left(j_{1}+j_{2}-J\right)}\left\langle j_{1} m_{1} j_{2} m_{2} \mid J M\right\rangle . \\
\left\langle j_{2} m_{2} j-m \mid j_{1}-m_{1}\right\rangle=(-1)^{j-j_{1}-m_{2}} \sqrt{\frac{2 j_{1}+1}{2 j+1}}\left\langle j_{1} m_{1} j_{2} m_{2} \mid j-m\right\rangle .
\end{gathered}
$$

Sendo $R$ uma rotação parametrizada pelos ângulos de Euler $\alpha, \beta, \gamma$,

$$
D_{m m^{\prime}}^{j}(R)=\mathrm{e}^{-i \alpha m} d_{m m^{\prime}}^{j}(\beta) \mathrm{e}^{-i \gamma m^{\prime}} .
$$

Para duas rotações $R_{2}, R_{1}$ sucessivas,

$$
\begin{gathered}
D_{m m^{\prime}}^{j}\left(R_{2} R_{1}\right)=\sum_{m^{\prime \prime}} D_{m m^{\prime \prime}}^{j}\left(R_{2}\right) D_{m^{\prime \prime} m^{\prime}}^{j}\left(R_{1}\right) . \\
D_{m_{1} m_{1}^{\prime}}^{j_{1}}(R) D_{m_{2} m_{2}^{\prime}}^{j_{2}}(R)=\sum_{j m m^{\prime}}\left\langle j_{1} m_{1} j_{2} m_{2} \mid j m\right\rangle\left\langle j m^{\prime} \mid j_{1} m_{1}^{\prime} j_{2} m_{2}^{\prime}\right\rangle D_{m m^{\prime}}^{j}(R) .
\end{gathered}
$$




\section{Referências Bibliográficas}

[1] S. Seitz, P. Schneider, and J. Ehlers. Light propagation in arbitrary spacetimes and the gravitational lens approximation. Class. Quantum Grav., 11, 1994.

[2] H. Spohn. The Lorentz process converges to a random flight process. Commun. Math. Phys., 60:277-290, 1978.

[3] P. H. Reimberg and L. R. Abramo. CMB and random flights: temperature and polarization in position space. JCAP, 06(043), 2013.

[4] L. R. Abramo, P. H. Reimberg, and H. S. Xavier. CMB in a box: Causal structure and the Fourier-Bessel expansion. Phys. Rev D, 82:043510, 2010.

[5] P. H. Reimberg and L. R. Abramo. The Jacobi map for gravitational lensing: the role of the exponential map. Class. Quantum Grav., 30:065020, 2013.

[6] P. H. Reimberg and L. R. Abramo. Extended random flights, Fourier-Bessel series, and a completeness-like relation for spherical Bessel functions. arXiv:1310.1128.

[7] V. Perlick. Einstein's Field Equations and their Physical Implications: Selected Essays in Honour of Jurgen Ehlers, volume 540 of Lectures Notes in Physics, chapter Gravitational Lensing from a Geometric Viewpoint. Springer, 2000.

[8] V. Perlick. On Fermat's principle in general relativity: II. The conformally stationary case. Class. Quantum Grav., 7, 1990.

[9] J. K. Beem and P. E. Ehrlich. Global Lorentzian Geometry. Dekker, 1981.

[10] S. Frittelli and E. T. Newman. Exact universal gravitational lensing equation. Phys. Rev. D, 59, 1999.

[11] V. Perlick. Exact gravitational lens equation in spherically symmetric and static spacetimes. Phys. Rev D, 69, 2004.

[12] V. Perlick. On Fermat's principle in general relativity: I. The general case. Class. Quantum Grav., 7, 1990.

[13] Gelfand I, M and S. V. Fomin. Calculus of Variations. Dover, 2000.

[14] V. Perlick. Ray Optics, Fermat's Principle, and Applications to General Relativity. Springer, 2010.

[15] S. W. Hawking and G. F. R. Ellis. The large scale structure of space-time. Cambridge U. P., 1973. 
[16] B. O'Neill. Semi-Riemannian Geometry. Academic Press, 1983.

[17] M. do Carmo. Geometria Riemanniana. Projeto Euclides, 2005.

[18] K. Uhlenbeck. A Morse Theory for geodesics on a Lorentz Manifold. Topology, 14, 1975.

[19] F. Giannoni, A. Masiello, and P. Piccione. A Morse Theory for light rays on stably causal Lorentzian manifolds. Ann. Inst. Henri Poincaré, 69, 1998.

[20] R. S. Palais. Morse Theory on Hilbert Manifolds. Topology, 2, 1963.

[21] P. Schneider, J. Ehlers, and E. Falco. Gravitational Lensing. Springer, 1992.

[22] V. Perlick. Criteria for multiple imaging in Lorentzian manifolds. Class. Quantum Grav., 13, 1996.

[23] A. Vilenkin. Gravitational field of vacuum domain walls and strings. Phys. Rev D, 23(4), 1981.

[24] A. Vilenkin and E. P. S. Shellard. Cosmic Strings and Other Topological Defects. Cambridge U. P., 1994.

[25] R. Geroch and J. Traschen. Strings and other distributional sources in general relativity. Phys. Rev D, 36(4), 1987.

[26] V. Perlick. Gravitational Lensing from a Spacetime Perspective. Living Rev. Relativity, 7(9), 2004.

[27] J. Ehlers. Relativistic kinetic theory. In B. K. Sachs, editor, Proceedings of the International School of Physics Enrico Fermi, volume 47. Academic Press, 1971.

[28] R. H. McKenzie. A gravitational lens produces an odd number of images. J. Math. Phys., 26, 1985.

[29] J. Milnor. Morse Theory. Princenton University Press, 1969.

[30] E. L. Lima. Curso de Análise, volume 2. Projeto Euclides, 1981.

[31] M. Morse. The calculus of variations in the large. AMS, 1934.

[32] T. Frankel. The Geometry of Physics. Cambridge U. P., second edition, 2009.

[33] F. Laudenbach. Transversalité, Courants et Théorie de Morse. Les éditions de l'école polytechnique, 2012.

[34] R. Geroch. Mathematical Physics. Chicago, 1985.

[35] P. Alexandroff. Elementary concepts of Topology. Dover, 1960.

[36] M. Nakahara. Geometry, Topology, and Physics. Taylor \& Francis, 2003.

[37] E. Witten. Supersymmetry and Morse theory. J. Differential Geometry, 17, 1982. 
166 Referências Bibliográficas

[38] M. Pettini. Geometry and Topology in Hamiltonian Dynamics and Statistical Mechanics. Springer, 2006.

[39] C. Nash and S. Sen. Topology and Geometry for Physicists. Academic Press, 1983.

[40] L. van Hove. The Ocurrence of Singularities in the Elastic Frequency Distribution of a Crystal. Physical Review, 89(6), 1953.

[41] R. Wald. General Relativity. University of Chicago Press, 1984.

[42] J. C. A. Barata. Curso de física-matemática. http://denebola.if.usp.br/ jbarata/Notas_de_aula/notas_de_aula.html.

[43] J K Beem, P E Ehrlich, and K L Easley. Global Lorentzian Geometry. Dekker, 2 edition, 1996.

[44] M. Bartelmann. Gravitational Lensing. Class. Quantum Grav., 27:233001, 2010.

[45] F Bernardeau, C. Bonvin, and F. Vernizzi. Full-sky lensing shear at second order. Phys. Rev D, 81:083002, 2010.

[46] A. O. Petters, H. Levine, and J. Wambsganss. Singularity Theory and Gravitational Lensing. Birkhäuser, 2001.

[47] M. Bartelmann and P. Schneider. Weak Gravitational Lensing. Physics Reports, 340, 2001.

[48] A. Lewis and A. Challinor. Weak gravitational lensing of the CMB. Physics Reports, 429, 2006.

[49] R. Durrer. The Cosmic Microwave Background. Cambridge, 2008.

[50] J. P. Uzan and P. Peter. Cosmologie Primordiale. Belin, 2005.

[51] V. Mukhanov. Physical principles of cosmology. Cambridge, 2005.

[52] P. H. Reimberg. Polarização da radiação cósmica de fundo. Master's thesis, Universidade de São Paulo, http://www.teses.usp.br/teses/disponiveis/43/43134/tde-06052010120027/es.php, 2009.

[53] G. N. Watson. A Treatise on the Theory of Bessel Functions, 2nd ed. Cambridge, 1944.

[54] B. D. Hughes. Random Walks and Random Environments. Clarendon Press, 1995.

[55] J. Dutka. On the problem of random flights. Archive for History of Exact Sciences, 32(3):351-375, 1985. 
[56] S. Chandrasekhar. Stochastic problems in physics and astronomy. Rev Modern Phys, 15(1):1-89, 1943.

[57] C. Pitrou. The radiative transfer at second order: a full treatment of the Boltzmann equation with polarization. Class. Quantum Grav., 26(6):065006, 2009.

[58] R. Penrose. Techniques of Differential Topology in General Relativity. CBMS-NSF, 1987.

[59] M. Kac. A Stochastic Model Related to the Telegrapher's Equation. Rocky Montain Journal of Mathematics, 4(3), 1974.

[60] A. D. Kolesnik and A. F. Turbin. The equation of symmetric Markovian random evolution in a plane. Stochastic Processes and their Applications, 75, 1998.

[61] A. D. Kolesnik. A four-dimensional random motion at finite speed. J. Appl. Prob., 43, 2006.

[62] A. D. Kolesnik. Random Motions at Finite Speed in Higher Dimensions. J. Stat. Phys., 131, 2008.

[63] E. Merzbacher, J. Feargin, and Wu T-H. Superposition of the radiation from $\mathrm{N}$ independent sources and the problem of random flights. Am. J. Phys., 45, 1977.

[64] H. Hochstadt. The Functions of Mathematical Physics. Dover, 1986.

[65] M. Franceschetti. When a random walk of fixed length can lead uniformly anywhere inside a hypershere. Journal of Statistical Physics, 127(4):813-23, 2007.

[66] M. Carmeli. Group theory and general relativity. McGraw-Hill, 1974.

[67] V. Perlick. Theoretical Gravitational Lensing - Beyond the weak-field smallangle approximation. arXiv:0708.0178v1.

[68] P. Cartier and C. deWitt Morette. Functional Integration. Cambridge U. P., 2006.

[69] R. K. Sachs and H. Wu. General Relativity for Mathematicians. Springer, 1977.

[70] M. Victoria and M. Sanchez. An Introduction to Lorentzian Geometry and its Applications. XVI Escola de Geometria Diferencial, IME, USP, 2010.

[71] R. Geroch. Domain of Dependence. J. Math. Phys., 11(2), 1970.

[72] A. Bernal and M. Sanchez. On Smooth Cauchy Hypersurfaces and Geroch's Splitting Theorem. Commun. Math. Phys., 243, 2003. 
168 Referências Bibliográficas

[73] A. Bernal and M. Sanchez. Smoothness of Time Functions and the Metric Splitting of Global Hyperbolic Spacetimes. Commun. Math. Phys., 257, 2005.

[74] M. Sanchez. A note on stability and Cauchy time functions. arXiv:1304.579\%v2, 2013.

[75] N. Straumann. General Relativity and Astrophysics. Springer, 2004.

[76] Y. Choquet-Bruhat. General Relativity and the Einstein Equations. Oxford, 2009

[77] Y. Choquet-Bruhat, C. deWitt Morette, and M. Dillard-Bleick. Analysis, Manifolds and Physics part 1. North-Holland, 1991.

[78] T. Pyne and M. Birkinshaw. Null geodesics in perturbed spacetimes. Astrophys. J., 415:459-468, 1993.

[79] J. D. Talman. Special Functions: A Group Theoretic Approach. W. A. Benjamin, Inc., 1968.

[80] R. P. Boas and H. Pollard. Complete sets of Bessel and Legendre functions. The Annals of Mathematics, 48(2):366-384, 1947.

[81] J. R. Higgins. Completeness and basis properties of sets of special functions. Cambridge University Press, 1977.

[82] J. N. Goldberg et al. Spin-s Spherical Harmonics and ð. J. Math. Phys., 8:2155, 1967.

[83] E. T. Newman and R. Penrose. Note on the Bondi-Metzner-Sachs Group. J. Math. Phys., 7:863, 1966.

[84] N. Straumann. From primordial quantum fluctuations to the anisotropies of the cosmic microwave background radiation. Annals. Phys. (Leipzig), 15, 2006.

[85] M. Zaldarriaga and U. Seljak. All-sky analysis of polarization in the microwave background. Phys. Rev. D, 55:1830-1840, 1997.

[86] L. C. Biedenharn and J. D. Louck. Angular Momentum in Quantum Physics - Theory and Applications. Addison-Wesley, 1981.

[87] G. F. Torres del Castillo. 3-D Spinors, Spin-Weighted Functions and their Applications. Birkhäuser, 2003.

[88] K. Gottfried and T. M. Yan. Quantum Mechanics: Fundamentals. Springer, 2003.

[89] M. Chaichian and R. Hagedorn. Symmetries in Quantum Mechanics. Taylor \& Francis, 1998. 